University of Louisville

ThinkIR: The University of Louisville's Institutional Repository

$12-2020$

\title{
The use of nanoparticles and electrospun fibers for intravaginal delivery to treat viral and bacterial infections and electrophysiological measurements of synthetic chloride channels.
}

Farnaz Minooei

University of Louisville

Follow this and additional works at: https://ir.library.louisville.edu/etd

Part of the Biochemical and Biomolecular Engineering Commons, and the Biomaterials Commons

\section{Recommended Citation}

Minooei, Farnaz, "The use of nanoparticles and electrospun fibers for intravaginal delivery to treat viral and bacterial infections and electrophysiological measurements of synthetic chloride channels." (2020). Electronic Theses and Dissertations. Paper 3559.

https://doi.org/10.18297/etd/3559

This Doctoral Dissertation is brought to you for free and open access by ThinkIR: The University of Louisville's Institutional Repository. It has been accepted for inclusion in Electronic Theses and Dissertations by an authorized administrator of ThinkIR: The University of Louisville's Institutional Repository. This title appears here courtesy of the author, who has retained all other copyrights. For more information, please contact thinkir@louisville.edu. 
THE USE OF NANOPARTICLES AND ELECTROSPUN FIBERS FOR INTRAVAGINAL DELIVERY TO TREAT VIRAL AND BACTERIAL INFECTIONS AND ELECTROPHYSIOLOGICAL MEASUREMENTS OF SYNTHETIC CHLORIDE CHANNELS

\author{
By \\ Farnaz Minooei \\ B.S. Sharif University, 2015 \\ A Dissertation Submitted to the Faculty of the \\ J. B. Speed School of Engineering University of Louisville \\ In Partial Fulfillment of the Requirements for the Degree of
}

\author{
Doctor of Philosophy \\ In Chemical Engineering \\ Department of Chemical Engineering \\ University of Louisville \\ Louisville, Kentucky
}

December 2020 

THE USE OF NANOPARTICLES AND ELECTROSPUN FIBERS FOR INTRAVAGINAL DELIVERY TO TREAT VIRAL AND BACTERIAL INFECTIONS AND ELECTROPHYSIOLOGICAL MEASUREMENTS OF SYNTHETIC CHLORIDE CHANNELS

By

Farnaz Minooei

A Dissertation Approved on

October 28, 2020

by the following Dissertation Committee:

Dr. Joel R. Fried

Dr. Jill M. Steinbach-Rankins

Dr. Hermann B. Frieboes

Dr. Gautam Gupta

Dr. Vance W. Jaeger

Dr. Kenneth E. Palmer 


\section{DEDICATION}

I dedicate this Ph.D. dissertation to my lovely parents, Mehrnoush and

Mahmoud Minooei, who always inspire me with their unconditional love, support, and encouragement. 


\section{ACKNOWLEDGMENTS}

Firstly, I would like to express my sincere gratitude to my advisors, Dr. Fried and Dr. Steinbach-Rankins for their guidance and help through each stage of the project. I also thank my advisors for providing me the opportunity to work in two distinct laboratories to acquire a variety of skills. I always appreciate their support, encouragement, and patience.

I would also want to thank Dr. Frieboes, Dr. Gupta, Dr. Jaeger, and Dr. Palmer for serving as my committee members and generously offering their time, guidance, and constructive feedback throughout my research.

I would acknowledge all of my colleagues and lab members in Dr. Fried and Dr. Steinbach-Rankins laboratories for their help, understanding, and friendship throughout my graduate studies including Kevin Tyo, Michael Martin, Longyun Zhang, Keegan Curry, Timothy Dubbs, and Abhinav Kanukunta.

Lastly, I express my warmest gratitude to my parents for their continuous love, help, and support and providing me the opportunity to travel oversee to pursue my passion and dreams. This journey would not have been possible without their encouragement and dedication. 


\begin{abstract}
THE USE OF NANOPARTICLES AND ELECTROSPUN FIBERS FOR INTRAVAGINAL DELIVERY TO TREAT VIRAL AND BACTERIAL INFECTIONS AND ELECTROPHYSIOLOGICAL MEASUREMENTS OF SYNTHETIC CHLORIDE CHANNELS
\end{abstract}

Farnaz Minooei

October 28, 2020

Female reproductive viral and bacterial infections affect millions of women worldwide. Given the diversity and magnitude of these unmet reproductive health challenges, topical administration of antiretrovirals (ARVs) and antibiotics have emerged as promising approaches to maintain and restore reproductive health. However, currently available intravaginal dosage forms often suffer from low user adherence and the need for frequent, daily administration to achieve therapeutic effect. To address these challenges, the broad goal of this research was to focus on the development of new localized nanoparticle (NP) and electrospun fiber dosage forms to prolong the delivery and enhance the efficacy of active agents to treat viral and bacterial infections.

The first goal of this work was to evaluate the synergistic interactions between a biologic, Q-Griffithsin (Q-GRFT), and three ARVs - tenofovir 
(TFV), raltegravir (RAL), and dapivirine (DAP) - in free and encapsulated forms, to identify unique protein-drug synergies to prevent human immunodeficiency virus type 1 (HIV-1) infection. Free Q-GRFT and free ARV co-administration resulted in strong synergistic interactions, relative to administration of each active agent alone. Similarly, Q-GRFT NP and ARV NP co-administration resulted in synergy across all formulations, with the most potent interactions between encapsulated Q-GRFT and DAP. This work suggests that Q-GRFT and ARV co-administration in free or encapsulated forms may improve efficacy and decrease the dose required to achieve prophylaxis. Moreover, the encapsulation of different active agents in NP-based platforms may provide modest levels of sustained-release with utility to a variety of agents and infection types.

The second part of this dissertation focused on the use of molecular dynamics (MD) and molecular mechanics simulations to study the compatibility of the mentioned ARVs with PLGA NPs. Solubility parameters were calculated for water, polymer, and each drug individually, and were compared with those attained from a group-contribution method (GCM). In addition, plots of the radial distribution function (RDF) and calculated charges obtained from electrostatic potential (ESP) fitting were used to compare the interactions between each drug and the polymer. Results indicated stronger hydrogen bonding between RAL and PLGA compared to TFV and PLGA. These findings explain the experimental observation that PLGA NPs encapsulating RAL have significantly higher encapsulation efficiency relative to NPs encapsulating TFV. This result provides important insight into the role of 
drug-polymer interactions on the encapsulation efficacy of small molecule antiretrovirals in polymeric NPs.

The third goal of this dissertation was to develop a new electrospun fiber dosage form to promote vaginal microbiota health, with the potential to prolong probiotic delivery for bacterial vaginosis (BV) treatment. First, we examined the initial safety and efficacy of fast-dissolving polyethylene oxide (PEO) fibers formulated alone or with an antibiotic in an established murine model of BV infection. We then fabricated PEO and polyvinyl alcohol (PVA) fibers containing Lactobacillus acidophilus (L. acidophilus) as a model probiotic. In addition, different parameters including electrospinning solution, the use of fresh or lyophilized bacteria, and extended storage conditions were evaluated for their impact on $L$. acidophilus viability and fiber morphology. Our results show that probiotics are highly and viably incorporated in PEO and PVA fibers, and exhibit prolonged stability for up to 3 months within -20 or $4^{\circ} \mathrm{C}$ storage conditions. In addition, this study suggests that blank and antibiotic-containing PEO fibers are safe in vivo, inert to the vaginal mucosa in the absence and presence of Gardnerella vaginalis (G. vaginalis) infection, and capable of delivering effective therapeutics. In addition, probiotics were highly and viably incorporated in PEO and PVA fibers, and exhibited prolonged stability for up to 3 months within -20 or $4^{\circ} \mathrm{C}$ storage conditions. Furthermore, PEO and PVA fibers inhibited the viability and cell adhesion of G. vaginalis, in both soluble and epithelial-based co-culture assays, suggesting their ability to exert health-promoting effects against pathogenic species involved in BV. 
Lastly, we sought to build upon the baseline hydrophilic rapid release fiber dosage form to develop a fiber-based sustained-release delivery platform to prolong probiotic release for up to 2 weeks. Two different fiber architectures mesh and layered - were developed to incorporate two lactic acid-producing model organisms, Lactobacillus crispatus (L. crispatus) and Lactobacillus acidophilus (L. acidophilus). In this study, fiber mass loss and morphology were assessed to evaluate fiber degradation over 2 wk, followed by the assessment of probiotic release and proliferation, lactic acid release, and changes in $\mathrm{pH}$. Lastly, the efficacy of these fibers was evaluated in an in vitro soluble co-culture assay against G. vaginalis infection. Both fiber architectures prolonged probiotic release for up to $14 \mathrm{~d}$ and produced therapeutically-relevant levels of lactic acid, which correlated with a significant reduction in $\mathrm{pH}$. Moreover, probiotic-containing fibers showed similar inhibitory properties to free probiotics against $G$. vaginalis, indicating that probiotics maintain their activity after electrospinning and have the potential to fully inhibit $G$. vaginalis infection. This study demonstrated that electrospun fibers composed of both hydrophilic and hydrophobic polymers may offer a viable longterm alternative to daily administration to maintain vaginal health, treat $B V$, and prevent BV recurrence. 
TABLE OF CONTENT

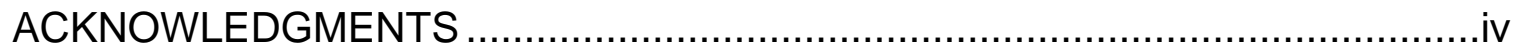

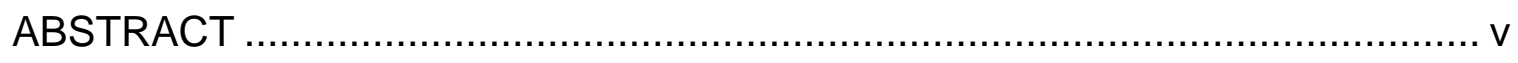

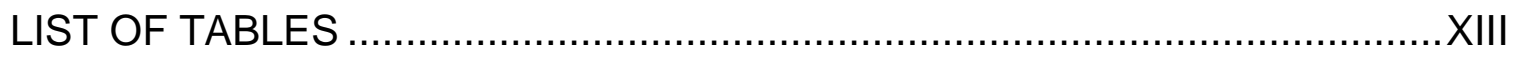

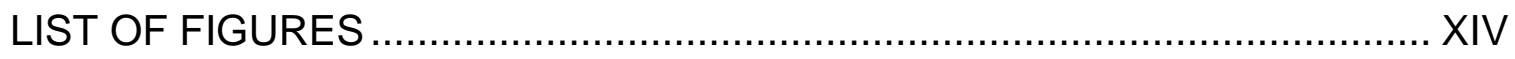

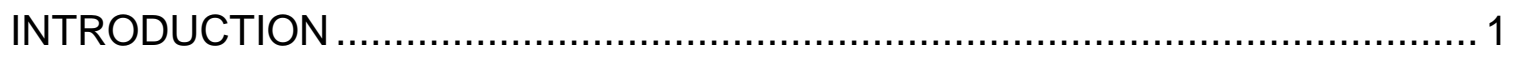

CHAPTER 1: RELATING ADVANCED ELECTROSPUN FIBER ARCHITECTURES TO THE TEMPORAL RELEASE OF ACTIVE AGENTS TO MEET THE NEEDS OF NEXT-GENERATION INTRAVAGINAL DELIVERY

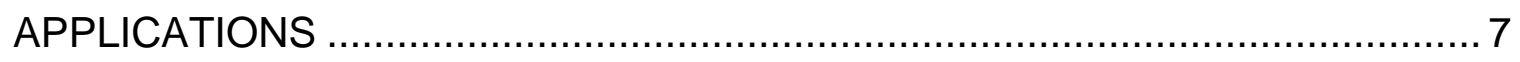

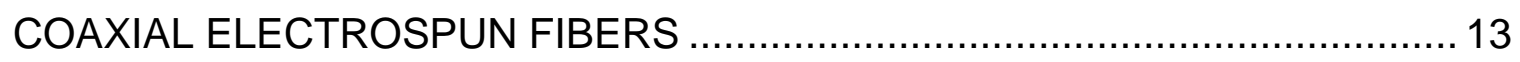

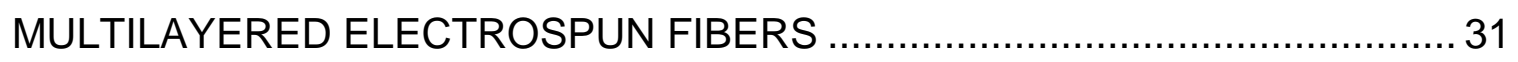

COMPOSITE NANOPARTICLE-FIBER DELIVERY VEHICLES ...................... 40

FUTURE DIRECTIONS AND DISCUSSION ................................................ 47 
CHAPTER 2: IN VITRO STUDY ON SYNERGISTIC INTERACTIONS BETWEEN FREE AND ENCAPSULATED Q-GRIFFITHSIN AND ANTIRETROVIRALS

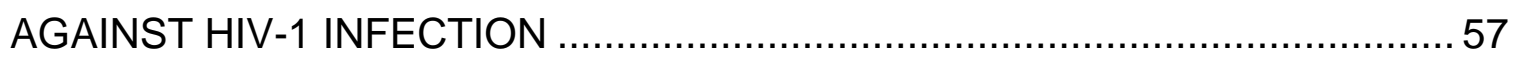

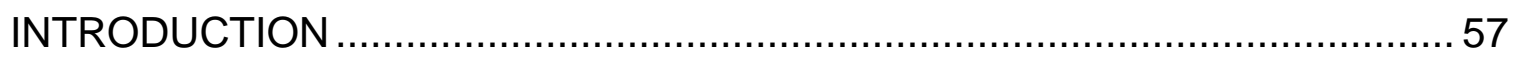

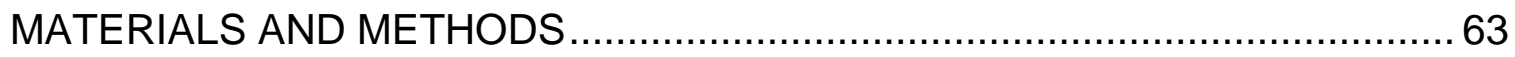

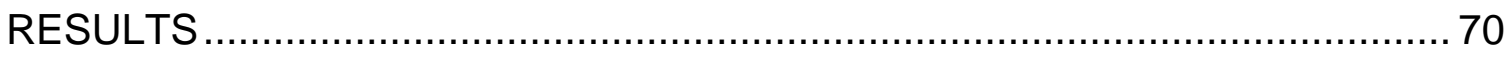

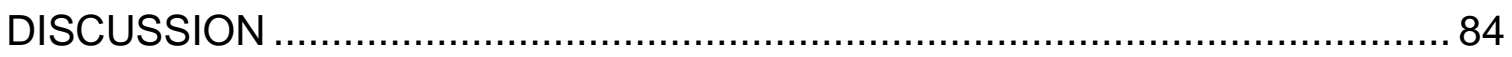

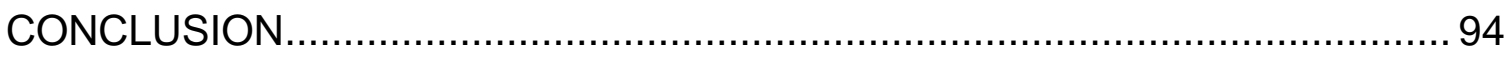

CHAPTER 3: A COMPUTATIONAL STUDY OF INTERACTIONS BETWEEN ANTIRETROVIRALS AND PLGA NANOPARTICLES ................................... 95

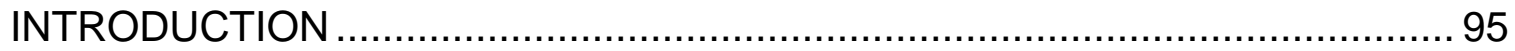

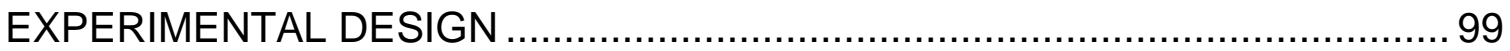

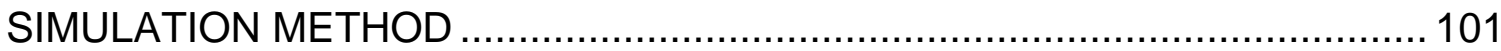

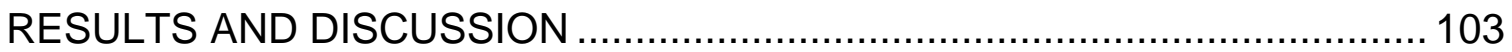

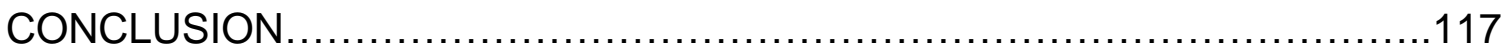

CHAPTER 4: PROBIOTIC FIBERS AS AN ALTERNATIVE DOSAGE FORM FOR

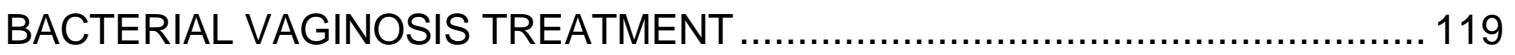

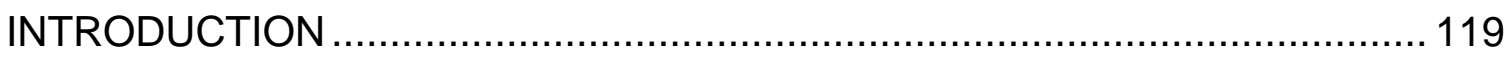


CHAPTER 5: MESH AND LAYERED FIBER ARCHITECTURES AS NOVEL PLATFORMS TO SUSTAIN PROBIOTIC RELEASE AGAINST BACTERIAL VAGINOSIS INFECTION 168

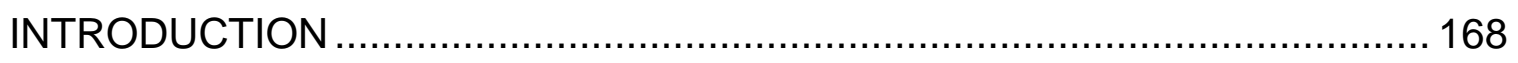

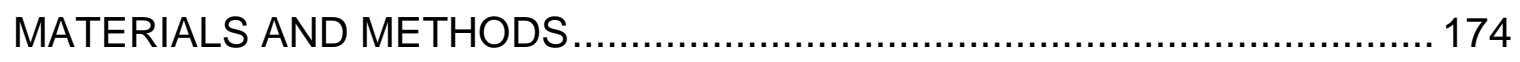

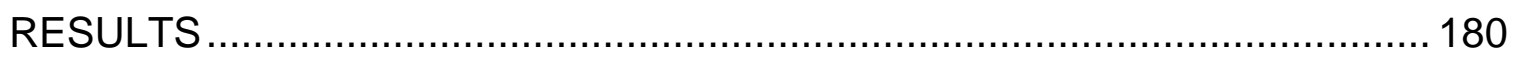

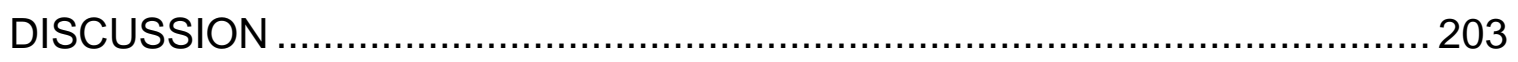

CHAPTER 6: FIRST ELECTROPHYSIOLOGICAL MEASUREMENTS CONFIRM SUCCINYL LINKER ENHANCES PERFORMANCE OF HEPTAPEPTIDE

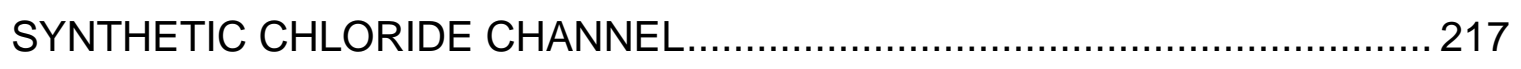

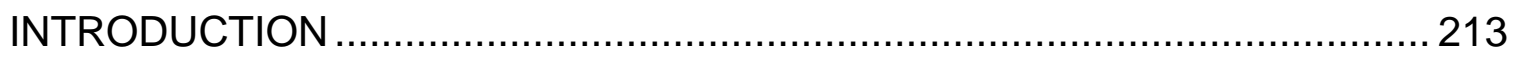

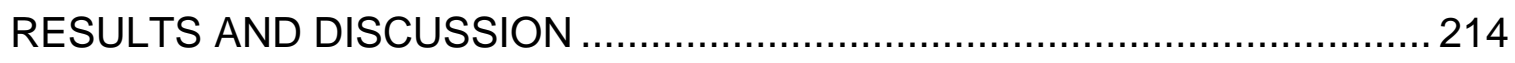

CHAPTER 7: OVERALL DISCUSSION AND CONCLUSION ........................221

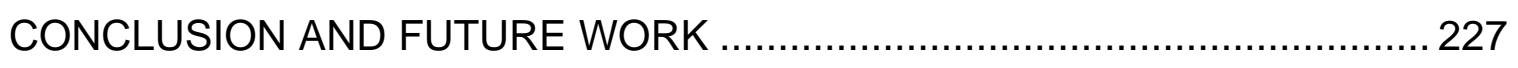

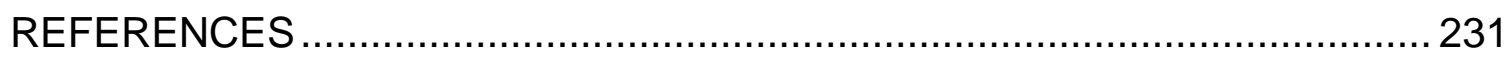




\section{LIST OF TABLES}

Table 2.1. Size, loading, and encapsulation efficacy of PLGA nanoparticles. Nanoparticles had theoretical loading values of $100 \mu \mathrm{g}$ Q-GRFT, TFV, RAL,

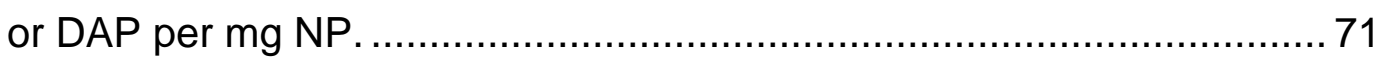

Table 2.2. $I_{50}$ values of free and encapsulated agents after administration to TZM-bl cells 1 hr before HIV-1 pseudovirus infection......................... 75

Table 2.3. Summary of the $\mathrm{IC}_{50}$ values of each individual active agent after

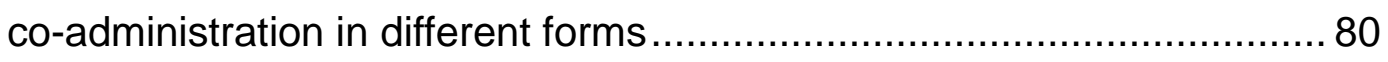

Table 3.1. Size, loading, and encapsulation efficiency (\%) of PLGA NPs

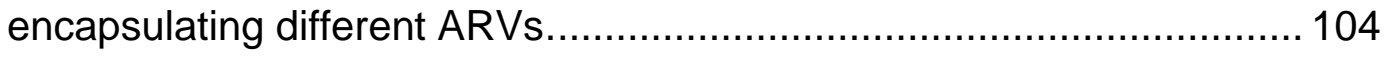

Table 3.2. Atomic charges for TFV, RAL, and DAP........................... 107

Table 3.3 Cohesive energy densities obtained from MD simulations and

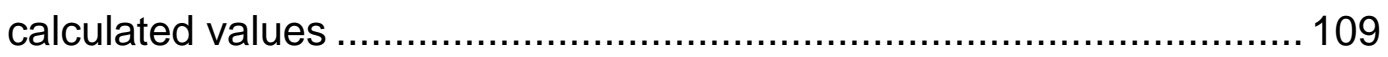

Table 4.1. G.v. and L.a. growth on different selective agar.................. 154 


\section{LIST OF FIGURES}

Figure 1.1. Schematic depicting examples of transient, short-term, and

sustained-release profiles.

Figure 1.2. Schematic of different electrospun fiber composites. 13

Figure 1.3. Schematic of anticipated release profiles from different coaxial fiber architectures. 14

Figure 1.4. Schematic of anticipated active agent release from multilayered fibers. 33

Figure 1.5. Schematic of electrospun nanoparticle-fiber composites that integrate coaxial and multilayered fiber architectures. 45

Figure 2.1. Scanning electron microscopy images of PLGA nanoparticles loaded with 10\% w/w (A) Q-GRFT, (B) TFV, (C) RAL, and (D) DAP....... 71

Figure 2.2. The cumulative release of $\mathrm{Q}-\mathrm{GRFT}, \mathrm{TFV}, \mathrm{RAL}$, and DAP from PLGA nanoparticles as a function of (A) total active agent release or $(B)$ the percent of total loading, after exposure to SVF for up to $14 \mathrm{~d}$ 72

Figure 2.3. The $\mathrm{IC}_{50}$ curves of $(\mathrm{A})$ free and (B) NP-encapsulated active agents after administration to TZM-bl cells $1 \mathrm{hr}$ prior to HIV-1 pseudovirus infection. 
Figure 2.4. The $I_{50}$ curves for free $A R V s$ and free Q-GRFT coadministration demonstrate synergistic interactions. 76

Figure 2.5. The $\mathrm{IC}_{50}$ curves for free drugs and Q-GRFT NP coadministration demonstrate synergistic interactions. 77

Figure 2.6. The IC 50 curves for ARV NP and Q-GRFT NP co-administration.

Figure 2.7. Combination indices of free Q-GRFT and free ARVs, Q-GRFT NPs and free ARVs, and Q-GRFT NPs and ARV NPs co-administered to TZM-bl cells. 81

Figure 2.8. (A) Schematic of NP-fiber composites in which spheres may depict Q-GRFT NPs or DAP NPs. 83

Figure 2.9. In vitro cytotoxicity of NPs encapsulating different agents administered to (A) VK2/E6E7 (B) End1/E6E7, and (C) Ect1/E6E7 cell lines using MTT assay. 84

Figure 3.1. Scanning electron microscopy images of PLGA nanoparticles loaded with 10\% w/w (A) Q-GRFT, (B) TFV, (C) RAL, and (D) DAP..... 104 Figure 3.2. Geometry-optimized structure of A) PLGA, B) TFV, C) RAL, and D) DAP 106

Figure 3.3. Identification of atoms participating in intermolecular interactions between drugs and water/polymer 
Figure 3.4. RDF for intermolecular interactions between A) oxygen atom (02s) in the ether group of PLGA and hydrogen atom (h10) in water; B) oxygen $(01=)$ in the carbonyl group of PLGA and oxygen $\left(02^{*}\right)$ in water; and C) hydrogen (h1) in PLGA and oxygen in water. 111

Figure 3.5. RDF for intermolecular interactions between hydrogen atom (h10) in TFV and oxygen atoms $\left(01=\right.$ or $\left.02^{*}\right)$ in A) PLGA in the absence of water; B) water in the absence of PLGA; C) PLGA in the presence of water and D) water in the presence of PLGA.

Figure 3.6. RDF for intermolecular interactions between hydrogen atom (h10) in RAL and A) oxygen atom (o1=) in PLGA in the absence of water; B) oxygen atom $\left(02^{*}\right)$ in water in the absence of PLGA; C) oxygen atom $(01=)$ in PLGA in the presence of water; and D) oxygen atom (02*) in water in the presence of PLGA 113

Figure 3.7. RDF for intermolecular interactions between hydrogen atom (h1n) in DAP and oxygen atom (01=) in A) PLGA in the absence of water; B) water in the absence of PLGA; C) PLGA in the presence of water; and D) water in the presence of PLGA. 114

Figure 3.8. Visualization of hydrogen bond formation between the oxygen atom ( $01=$ ) of PLGA and $\mathrm{A}$ ) hydrogen atom ( $\mathrm{h10}$ ) of TFV, B) hydrogen atom (h10) of RAL, and C) hydrogen atom (h1) of DAP. Hydrogen bonds are shown with red arrows. 116

Figure 4.1. PEO fibers (EFs) are safely delivered in the vagina. 137 
Figure 4.S1. Histological analysis of mouse vaginal tissue. 138

Figure 4.2. Evaluation of the ability of metronidazole-containing PEO fibers (EFs) to exert a therapeutic effect in a mouse model of BV using vaginal inoculation of $G$. vaginalis (G.v.). 140

Figure 4.3. L. acidophilus (L.a.) viability in different (A) PEO and (B) PVA formulations after electrospinning. 143

Figure 4.S2. SEM images of 5\% w/v PEO and 10\% w/v PVA electrospun fibers containing $5 \times 10^{7} \mathrm{CFU}$ L.a./mg polymer: (A through F) 5\% PEO fiber formulations; (G through L) 10\% PVA fiber formulations. 145

Figure 4.S3. The diameters of PEO and PVA fibers electrospun with $5 \times 10^{7}$ $\mathrm{CFU} / \mathrm{mg}$ fresh or lyophilized L.a. electrospun in water, MRS broth or MRS broth and glycerol.

Figure 4.4. SEM images of $5 \% \mathrm{w} / \mathrm{v}$ PEO and $10 \% \mathrm{w} / \mathrm{v}$ PVA fibers that were electrospun in water and contain different concentrations of fresh L.a. . 148

Figure 4.S4. The viability of L.a. in (A) PEO and (B) PVA fibers electrospun with $5 \times 10^{7} \mathrm{CFU}$ L.a./mg in water, MRS broth, or MRS broth with $5 \%$ glycerol, was evaluated after storage in $-20^{\circ} \mathrm{C}, 4^{\circ} \mathrm{C}$, and $25^{\circ} \mathrm{C}$ for up to 90 days after electrospinning. 150

Figure 4.5. L.a. incorporated in PEO and PVA fibers inhibits G.v. viability in soluble co-culture assays. 152

Figure 4.S5. L.a. and G.v. growth in NYC or MRS broth. 153 
Figure 4.S6. G.v. and L.a. viability after culture in either MRS or NYC III broth 154

Figure 4.6. L.a. incorporated in PEO and PVA fibers inhibit G.v. growth in epithelial cell co-culture competition assays 156

Figure 5.1. Schematic design of (A) mesh and (B) layered fiber architectures. 177

Figure 5.S1. Fluorescence microscopy images of different mesh fiber compositions as a result of dual-electrospinning with different PLGA (green) to PEO (red) flow rate ratios. 181

Figure 5.2. Probiotics are successfully incorporated within mesh PLGA:PEO (1:1) and PLGA:PEO (1:3) fiber architectures................... 183

Figure 5.S2. Probiotics are successfully incorporated within layered PLGA:PEO (1:1) and PLGA:PEO (1:3) fiber architectures. 184

Figure 5.3. Probiotic biofilms are formed on mesh fiber surfaces after $48 \mathrm{hr}$ and persist through $2 \mathrm{wk}$. 186

Figure 5S.3. Probiotic biofilms are formed on layered fiber surfaces after 48 $\mathrm{hr}$ and persist through 2 wk. 187

Figure 5.4.SEM images of mesh and layered blank fibers after $1 \mathrm{hr}, 48 \mathrm{hr}$, $1 \mathrm{wk}$, and 2 wk degradation time points 190 
Figure 5.5. Macrostructural images of blank mesh PLGA:PEO (1:1) fibers over the duration of the 3 wk degradation study. 191

Figure 5.6. The cumulative release and proliferation of L. crispatus (L.c.) and $L$. acidophilus (L.a.) from $(A, C)$ mesh and $(B, D)$ layered fibers over 2 wk.. 193

Figure 5.7. Lactic acid production from mesh (A, B, C, and D) and layered $(E, F, G$, and $H)$ fibers. 197

Figure 5.8. The $\mathrm{pH}$ of PLGA:PEO mesh and layered fiber eluates was measured over 2 wk. 200

Figure 5.9. L.c. PLGA:PEO (1:1) mesh or layered fibers were co-cultured with G.v. at equal initial concentrations of $10^{6} \mathrm{CFU} / \mathrm{mL}$ for 4,24 , and $48 \mathrm{hr}$. 202

Figure 6.1. Molecular structure of different compounds used in the experiment. 215

Figure 6.2. Current vs. time with a $100 \mathrm{~Hz}$ low pass Bessel filter (left) and histogram of conductance (right) for 4 at $-65 \mathrm{mV}$. 218

Figure 6.3. Current vs. time plot after replacing $\mathrm{KCL}$ with $\mathrm{K} 2 \mathrm{SO} 4$ in trans compartment. 219 


\section{INTRODUCTION}

Human immunodeficiency virus (HIV) remains a major health challenge, impacting over 1.7 million people each year ${ }^{1,2}$ and over 38 million worldwide. Women are disproportionately affected by HIV infections, and more than half of the world's infected population is comprised of women living in sub-Saharan Africa ${ }^{3}$. Currently, there is no cure for or effective vaccine against HIV- $1^{1}$, hence a major emphasis has been placed on developing strategies to prevent and treat HIV-1 infections. Furthermore, due to the increased risk of viral co-infections in HIV-1 infected patients, a primary focus has been to evaluate combinations of antiretroviral drugs delivered with multipurpose prevention technologies, to simultaneously target multiple sexually transmitted infections (STIs) ${ }^{4}$, such as herpes simplex virus 2 (HSV-2) and HIV-1.

Bacterial vaginosis $(\mathrm{BV})$ is the most common vaginal infection in women between the ages of 15 to $44^{5}$, impacting $~ 30 \%$ of women in the U.S. and globally 6 . Furthermore, bacterial vaginosis has been shown to increase the risk of HIV-1 and other virus acquisition in women by $60 \%{ }^{7}$. In addition, women co-infected with BV and HIV-1 have a higher intravaginal concentration of HIV-1 RNA, relative to HIV1-only infected women ${ }^{7}$. In BV, the normal microbial ecology of the reproductive tract is altered, and resident lactobacilli are replaced by an overgrowth of pathogenic vaginal anaerobes such as G. vaginalis, as well as Atopobium vaginae, 
Prevotella, Peptostreptococcus, and Bacteroides spp ${ }^{8-10}$. Infections related to BV may occur due to the abundance of BV-associated bacteria or other sexually transmitted pathogens. Women who are infected with BV are specifically susceptible to the acquisition of Trichomonas vaginalis, Neisseria gonorrhoeae, Chlamydia trachomatis, herpes simplex virus type 2 (HSV-2), and HIV-1. Furthermore, studies have shown that BV can exacerbate the spread of HIV-1 and HSV-2 by promoting viral replication and vaginal shedding of these viruses ${ }^{11}$.

Intravaginal delivery is an effective strategy to improve the localization of antiviral, antibacterial, antifungal, chemotherapeutic, and contraceptive agents within the female reproductive tract $(\mathrm{FRT})^{12,13}$. Relative to oral administration routes, intravaginal delivery localizes agents to the FRT, avoiding both the harsh gastrointestinal environment and hepatic first-pass effect. This results in an increase in active agent bioavailability within the target tissue and corresponding functional activity, by decreasing off-target effects and systemic exposure ${ }^{14}$. The inherent characteristics of the FRT, including its large surface area and low enzymatic activity, additionally make the FRT a favorable site for localized active agent administration and targeting ${ }^{15,16}$.

Although intravaginal delivery offers a variety of advantages to enhance the delivery of active agents ${ }^{17}$, challenges unique to the FRT must be overcome to provide efficacious prophylaxis and treatment. One of the most important components of the FRT is the mucus layer, which protects the epithelium and lamina propria from incoming pathogens. However, it can also act as a barrier, impeding therapeutic transport to underlying epithelial and immune cells ${ }^{18,19}$. In 
addition to these challenges, the frequent shedding and production of cervicovaginal mucus can decrease active agent retention, while bacterial flora, enzymes, and the acidic environment created by beneficial bacteria can contribute to metabolization and degradation of active agents, reducing efficaciousness.

To address these challenges, intravaginal delivery platforms have been formulated as solid or semi-solid dosage forms that include suppositories, tablets, capsules, gels, rings, and creams to enhance delivery to and retention in the $\mathrm{FRT}^{20-24}$. While these dosage forms have enabled high levels of active agent incorporation and localization, these traditionally used delivery platforms still face significant challenges, including difficulty of self-administration, economic feasibility, poor user compliance, vaginal irritation, the need for frequent administration, and low residence times ${ }^{25}$. Of these platforms, intravaginal rings have provided the "gold standard" for long-term delivery due to their ability to sustain the release of one or multiple active agents for weeks to months, avoid leakage and loss of active agent, and improve drug stability ${ }^{26-30}$. However, some biological agents have difficulty withstanding the high temperature and solvent processes often required for fabrication, limiting their incorporation ${ }^{31}$.

More recently, nanoparticles (NPs) have been developed for topical intravaginal delivery of antivirals and antimicrobials due to their ability to encapsulate both hydrophilic and hydrophobic agents and to overcome challenges such as agent instability, low cellular internalization, and tissue distribution ${ }^{32-40}$. However, NP administration can result in low intravaginal retention due to mucus shedding, or conversely may be immobilized within the mucus layer, resulting in 
inadequate transport to underlying target tissue ${ }^{40}$. To improve retention and to maximize transport, NPs have been surface-modified ${ }^{41,42}$, while carrier solutions with different osmolarities have been explored to increase retention within and penetration of the vaginal lumen ${ }^{43-45}$. Despite these efforts, hurdles including low encapsulation efficiency and rapid release of hydrophilic agents have hindered the ability to achieve long-term delivery and retention ${ }^{46}$. Given these issues, other delivery platforms have been investigated that may increase the longevity of active agents within the FRT and improve user adherence while also offering a new dosage form alternative to women.

Electrospun fibers have emerged as a relatively new delivery platform to improve active agent retention and delivery for intravaginal applications due to their ease of use, ability to be fabricated into various geometries and sizes, and tunable release properties ${ }^{47,48}$. They have been considered for sustained-delivery, a characteristic that is often desirable for intravaginal applications, due to their high surface-area-to-volume ratio, degree of interconnected porosity, tunable pore sizes, surface-modification potential, interchangeable polymer options, and diverse fiber architectures that enable finer control over the rate, duration, and site of agent release ${ }^{49}$. Electrospun fibers have the additional advantage that they can be fabricated using a variety of natural or synthetic polymers to tailor release properties ${ }^{50}$, and these polymer types are typically selected based on their biocompatibility, hydrophobicity, and related degradation properties.

The focus of this work was to develop delivery platforms comprised of nanoparticles and/or electrospun fibers to both enhance the efficacy of active 
agents and provide prolonged release (here defined for durations longer than 1 week). This dissertation includes 7 chapters that represent manuscripts that have been published, submitted, or will be submitted. Some content may naturally overlap the content of other chapters; therefore, some descriptions may be repeated for the sake of comprehensiveness. CHAPTER 1 comprises a review article which was published in Pharmaceutics and I shared co-first authorship with Dr. Kevin Tyo on this publication. This chapter reviews different fiber architectures that have been useful for active agent delivery and provides guidelines for the development of new formulations that exhibit release kinetics relevant to the time frames and the diversity of active agents needed in next-generation multipurpose applications. CHAPTER 2 discusses findings related to the synergistic interactions between ARVs and a biologic, GRFT, for HIV-1 prevention, and is in the process of submission. The computational study presented in CHAPTER 3 summarizes the study of molecular interactions between ARVs and polymeric nanoparticles and is in the process of submission. CHAPTER 4 presents research regarding the development of rapid-release probiotic fibers as an alternative dosage form for BV treatment. The corresponding manuscript was submitted to Science Advances. CHAPTER 5 describes our work to develop probiotic-containing sustained-release fibers for BV applications and is in preparation for submission. Lastly, CHAPTER 6 notes previous work in which the activity of synthetic chloride channels in different lipid systems was evaluated. This work was published in Chemical Communications. The dissertation concludes with CHAPTER 7 which briefly 
summarizes the findings of the previous chapters and provides future directions for each project. 


\section{CHAPTER 1}

\section{RELATING ADVANCED ELECTROSPUN FIBER ARCHITECTURES TO THE TEMPORAL RELEASE OF ACTIVE AGENTS TO MEET THE NEEDS OF NEXT-GENERATION INTRAVAGINAL DELIVERY APPLICATIONS}

Electrospun fibers have recently gained attention for intravaginal delivery due to their ease of use, ability to be fabricated into various geometries and sizes, and tunable release properties ${ }^{47,48}$. They have been considered for sustaineddelivery, a characteristic that is often desirable for intravaginal applications, due to their high surface area-to-volume ratio, degree of interconnected porosity, tunable pore sizes, surface-modification potential, interchangeable polymer options, and diverse fiber architectures that enable finer control over the rate, duration, and site of agent release ${ }^{49}$. Electrospun fibers have the additional advantage that they can be fabricated using a variety of natural or synthetic polymers to tailor release properties ${ }^{50}$, and these polymer types are typically selected based on their biocompatibility, hydrophobicity, and related degradation properties.

One of the most significant factors that contributes to active agent release from fibers is the relative hydrophobicity of the selected polymer material ${ }^{51,52}$. In addition to polymer hydrophobicity, the medium (in vitro) or environment (in vivo) surrounding the fiber can impact drug release. Simulated vaginal and seminal 
fluids, often used to preliminarily assess intravaginal release, may alter the release of agents relative to testing in water or phosphate buffered saline (PBS) (in vitro) or in vivo, due to differences in viscosity, salt, and protein concentrations, as well as $\mathrm{pH}$. Therefore, depending on the degree of polymer hydrophobicity and the environment release it is tested in, the same encapsulated active agent can have distinctly different release profiles, in some cases ranging from hours to months ${ }^{53,54}$. Usually, independent of these conditions, the use of hydrophilic polymers often results in the immediate release of both hydrophilic and hydrophobic active agents due to the high solubility and degradation rate of hydrophilic polymers in aqueous environments ${ }^{55}$. Natural polymers such as collagen, gelatin, chitosan, elastin, and laminin, and synthetic polymers including poly(ethylene oxide) (PEO), polyvinyl alcohol (PVA), and polyvinylpyrrolidone (PVP) are examples of hydrophilic materials that have been fabricated into fibers with micron- and nanometer-scaled properties. In contrast, synthetic hydrophobic polymers including polycaprolactone (PCL), poly(lactic-co-glycolic acid) (PLGA), and polyurethane (PU) have demonstrated burst or sustained-release kinetics depending on the hydrophobicity of the incorporated active agent ${ }^{56-60}$. Moreover, synthetic hydrophobic polymers can also serve as a mechanical and structural basis for different fiber architectures in which the release of single or multiple encapsulants may be tailored by using more complex fiber designs or composites. Fiber release rates can also be optimized by adjusting the polymer molecular weight or hydrophilicity, for example, by adding hydrophilic groups such as aliphatic poly(phosphoester) to the polymer structure ${ }^{61}$. Together, these features 
have enabled the incorporation and release of a variety of antiviral, antimicrobial, and biological agents from fiber scaffolds ${ }^{62-64}$.

Active agent release from polymeric fibers typically occurs via diffusion, polymer degradation, and erosion ${ }^{52}$. When fibers are first administered, solvent or solution diffuses through the porous fiber matrix. Once in contact with the solvent or solution, the polymer matrix swells, loosening polymer chains and enabling the diffusion of active agents, dependent in part on molecular size. Concurrently, the fiber surface may undergo bulk erosion at a rate corresponding to polymer hydrophilicity. These features in combination with the large surface-to-volume ratio of the fibers allows for the increased diffusion of encapsulants relative to diffusion from non-porous bulk materials ${ }^{65}$. Traditionally, fibers have been electrospun as uniaxial fibers or fibers that comprise a single polymer or polymer blend and exhibit homogeneous morphology. Diffusion of active agents from more traditional uniaxial fibers is dependent upon the compatibility of the encapsulant, polymer, and surrounding eluant. In contrast with diffusion, polymer degradation is observed when fibers are exposed to aqueous environments, and polymer bonds are cleaved by either passive hydrolysis or enzymatic reaction ${ }^{66}$, resulting in slow degradation of the fiber scaffold. This degradation alters the distance between and size of interconnected pores, thereby impacting the diffusion and release of incorporated active agents. For most synthetic polymers, hydrolysis is the most common mechanism of degradation, although hydrolysis-resistant polymers have been utilized ${ }^{67}$, which significantly impact active agent release. As the fibers degrade, they can also undergo surface or bulk erosion, which is dependent upon 
solvent diffusivity into the fiber, polymer solubility, and overall fiber matrix dimensions ${ }^{68}$.

As a result of these mechanisms and the materials selected, electrospun fibers can tailor the release of encapsulated agents within different durations to achieve immediate (transient or rapid), short-term, or sustained-release. Within this review, we defined release as transient, when the complete release of active agents occurs within $24 \mathrm{~h}$ of administration; short-term, when the release occurs from one day to one week; or sustained, when the release of the active agent occurs over a duration of weeks to months. A schematic showing an example of these different potential release profiles is provided in Figure 1.1. Factors including the electrospinning parameters, polymer materials, fiber architecture, the resulting structure and morphology, and the distribution and amount of incorporated active agent each contribute to the resulting release kinetics and efficacy of delivery ${ }^{48}$. 

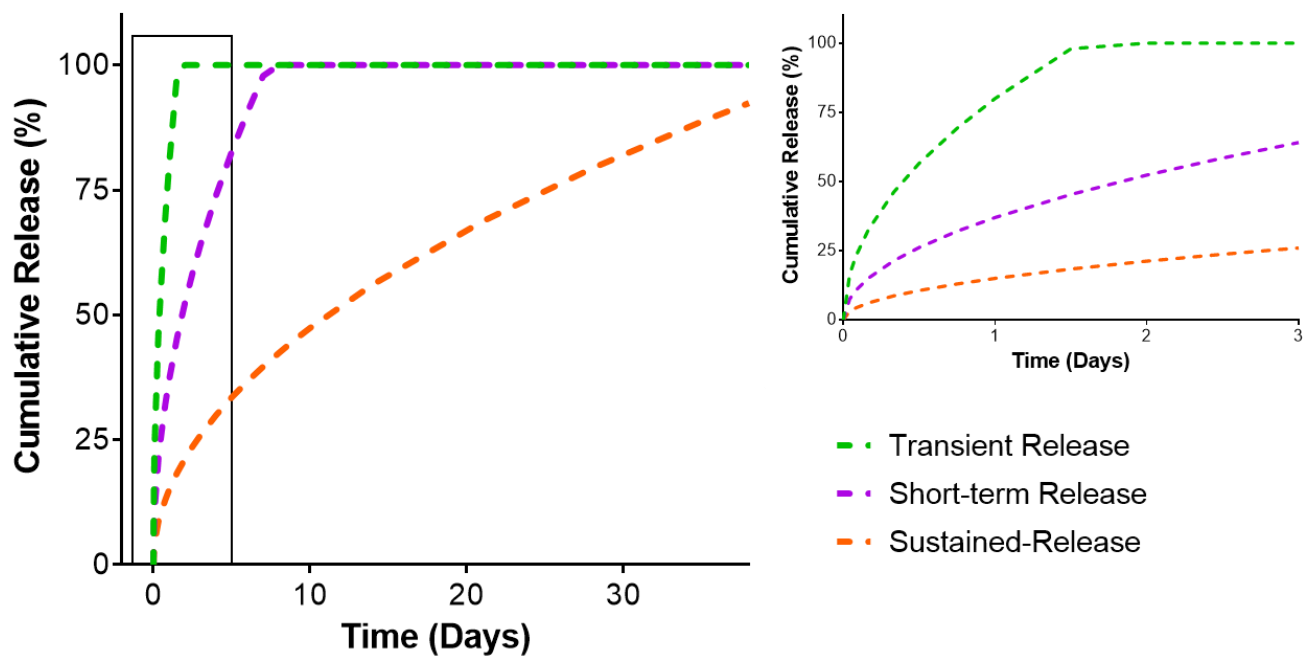

- Transient Release

- Short-term Release

- Sustained-Release

Figure 1.1. Schematic depicting examples of transient, short-term, and sustainedrelease profiles.

Traditional uniaxial electrospun fibers in which each individual fiber is composed of a single cohesive polymer layer were the first fiber architectures to be fabricated ${ }^{69}$ and have been utilized in a variety of drug delivery applications over the past decade ${ }^{47,48,70-72}$. While uniaxial fibers offer high encapsulation efficiencies, cost-effectiveness, and ease of use, they have suffered from burst release and challenges in tailoring release properties ${ }^{48,53,73}$. These challenges are most evident in achieving the sustained-release of hydrophilic agents, often necessitating hydrophilic polymers to attain high encapsulation efficacy as well as hydrophobic polymers for sustained-release. More complex fiber architectures offer alternative options to address these limitations by combining different polymer types in distinct layers to modulate the release.

While the release characteristics of traditional uniaxial electrospun fibers have been thoroughly reviewed in literature ${ }^{74-79}$, to our knowledge, there has not 
yet been a review of the more advanced fiber architectures used to deliver active agents, nor a review that considers the impact these architectures may have on intravaginal delivery applications. Here, we seek to provide an overview of different polymer architectures including coaxial, multilayered, and nanoparticle-fiber composites (Figure 1.2) as a function of the materials used to construct these architectures that have been utilized in a diversity of health applications. We seek to present different material combinations in these architectures to systematically relate material type and fiber architecture to active agent release kinetics. Last, we explore how lessons derived from these different architectures might be applied in the context of intravaginal delivery to address the needs of future topical sustainedrelease platforms for a given prophylactic or therapeutic application. The overall goal of this review is to provide a summary of different fiber architectures that have been useful for active agent delivery and to provide guidelines for the development of new formulations based on the knowledge obtained from previous work across other applications. While some of these more complex architectures have only recently been investigated relative to uniaxial fibers, they have demonstrated promise in enabling greater tunability of release and may be useful to apply as new dosage forms for intravaginal delivery and other similar applications. 
A

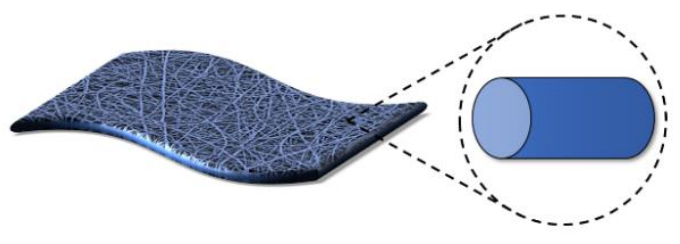

C

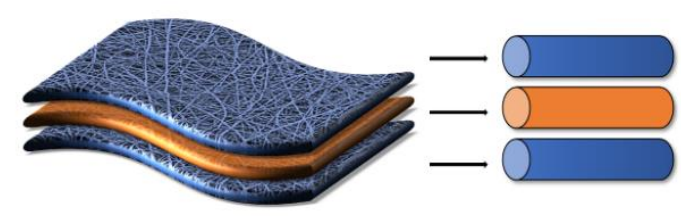

B

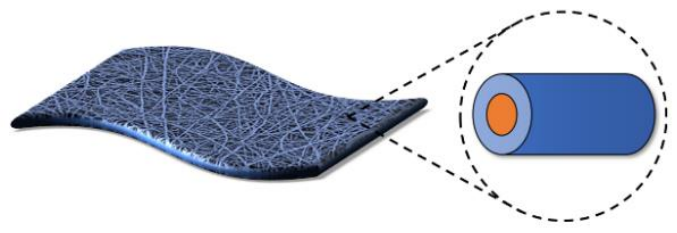

D

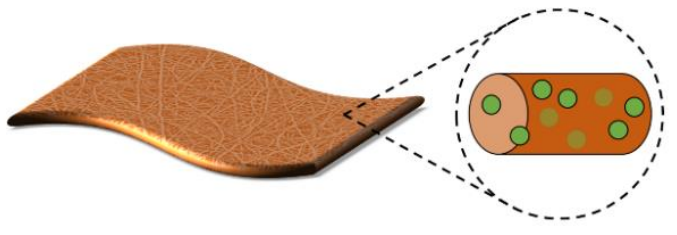

Figure 1.2. Schematic of different electrospun fiber composites. Diagrams representing (A) traditional uniaxial fibers, (B) coaxial fibers, (C) multilayered fibers, and (D) nanoparticle-fiber composites. (A) Uniaxial fibers are comprised of a single polymer or polymer blend (shown in blue) that is distributed homogenously throughout the fiber structure. (B) In contrast, coaxial fibers contain both core (orange) and shell (blue) layers that are chemically distinct. (C) Multilayered fibers result from sequentially electrospinning different fiber layers together or integrating individual layers post-fabrication. (D) Finally, nanoparticle-fiber composites consist of hydrophilic or hydrophobic fibers (orange) that encapsulate nanoparticles (green).

\section{Coaxial Electrospun Fibers}

\section{Coaxial Architectures and Properties}

Coaxial electrospinning, adapted from uniaxial or single axial electrospinning, provides a multicomponent fiber scaffold that easily allows the tunable release of active agents ${ }^{80,81}$. Coaxial fibers are usually comprised of two parts, an outer protective layer or shell and an inner layer or core ${ }^{82}$, where 
encapsulants are typically localized (Figures 1.3B and 1.4). Coaxial fibers can provide several advantages relative to uniaxially spun fibers. First, electrospinning the core and shell polymer solutions simultaneously through a coaxial spinneret allows for the design of unique fiber architectures. The thickness and ratios of the core and shell layers can be modulated, providing more reproducible fiber properties with a greater ability to alter encapsulant release relative to other fabrication methods. Additionally, coaxial electrospinning ensures that the active agent in the core phase is protected within harsh physiological environments, such as the female reproductive tract ${ }^{64}$. Furthermore, a variety of materials can be used as either the core or shell to finely regulate encapsulant release (Figure 1.3) ${ }^{80,83}$.
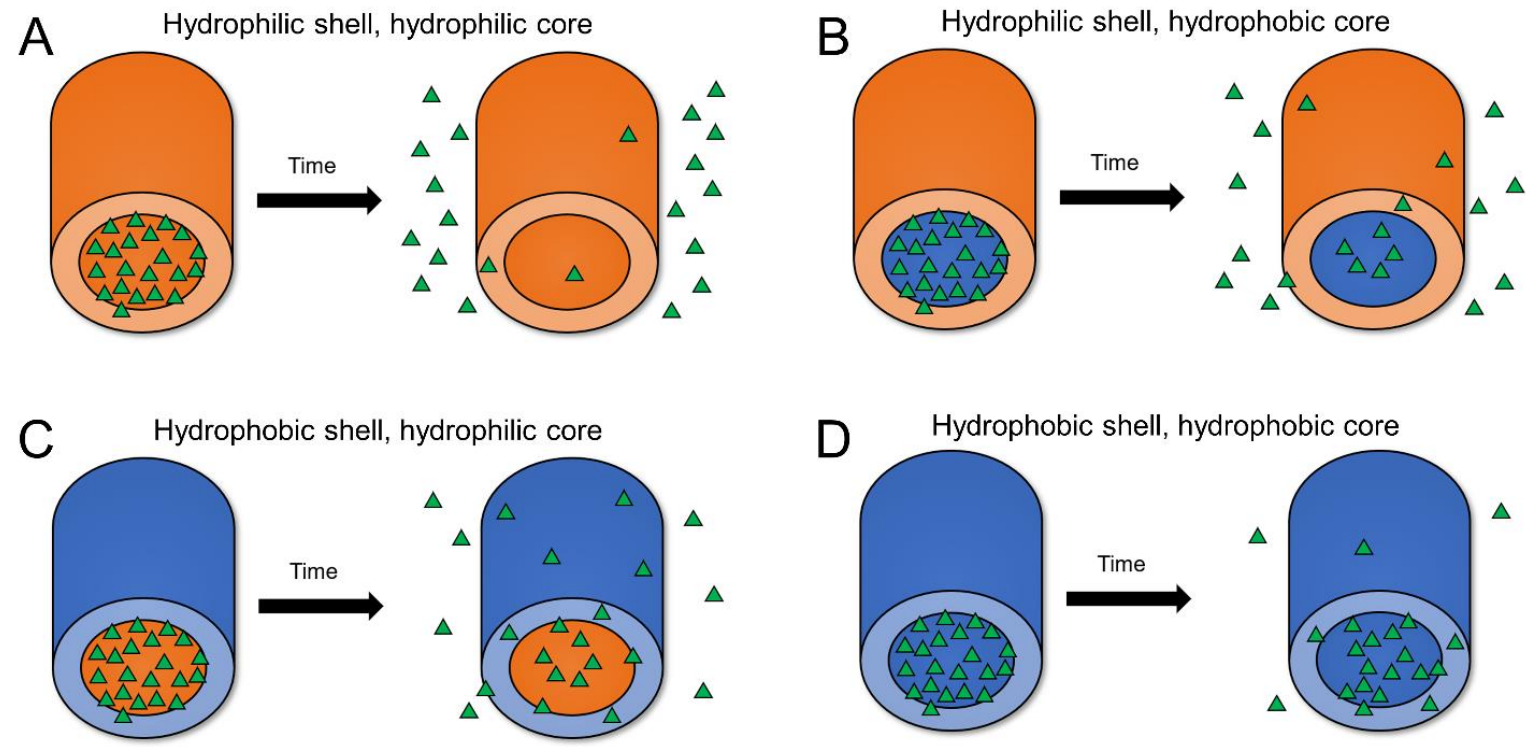

Figure 1.3. Schematic of anticipated release profiles from different coaxial fiber architectures.

Despite these advantages, the added complexity of simultaneously electrospinning two or more polymer phases and the additional interactions 
between the core and shell solutions requires additional optimization relative to uniaxial electrospinning in terms of selecting compatible polymers and solvents. In addition to the core-shell architecture itself, the release profiles of active agents from coaxial fibers are impacted by solvent choice, polymer-solvent miscibility, the miscibility between core and shell solvents/solutions, solvent volatility, and layer thicknesses ${ }^{84,85}$. Solvent choice has been shown to alter fiber diameter and structure $^{86}$, thereby impacting active agent release ${ }^{87}$. Additionally, miscible core and shell solvents/solutions may lead to the partial dissolution of core encapsulants in the shell, whereas, immiscible core and shell solvents may promote material delamination at the core-shell interface, facilitating burst release of the core encapsulant. Therefore, the polymers and solvents for both core and shell layers must be selected based on their individual properties as well as their anticipated interactions ${ }^{88,89}$. In addition, solvent volatility and evaporation rate can affect the distribution and subsequent release of active agents, while the thickness of the polymer shell, polymer composition, and spinning conditions influence encapsulant diffusion rates ${ }^{90}$. Here, we discuss coaxial fibers as a function of their core-shell design, composition, and incorporated active agents to help relate these considerations to the resulting transient, short-term, or sustained-release.

\section{Characteristics Release Kinetics from Coaxial Fibers}

\section{Transient Release (within $24 \mathrm{~h}$ )}

\section{Hydrophobic Shell—Hydrophilic Core}


Electrospun fibers can be designed to release the active agent immediately or within $24 \mathrm{~h}$ of administration if a rapid onset of action is needed for a given application ${ }^{91}$. Moreover, multiple active agents can be incorporated into different layers of a coaxial fiber (core or shell) to provide transient release.

For application to infectious diseases, coaxially spun fibers that demonstrate burst release followed by lower levels of short-term release may provide on-demand protection against incoming pathogens, increasing the immediate efficaciousness of agents by releasing initially high (burst) concentrations. This type of release can be achieved by employing coaxial fibers comprised of hydrophobic shells and hydrophilic cores. In one study, coaxial and triaxial fiber multi-drug delivery platforms that used PCL as the outermost shell released $\sim 15 \%$ and $\sim 80 \%$ of two different hydrophilic dyes, keyacid blue and keyacid uranine (KAB and KAU), from the PVP core and PCL shell fibers, respectively, within one hour ${ }^{80}$. In both the coaxial and triaxial fibers, the PVP core containing $\mathrm{KAB}$ was protected by the surrounding PCL layer containing KAU, which helped to extend the release of the remaining $K A B$ to $24 \mathrm{~h}$. For the triaxial fibers, a blank PCL layer was electrospun between the outer PCL shell and the inner PEO core. In both the coaxial and triaxial fibers, KAU was released from the shell within $3 \mathrm{~h}$; however, the triaxial fibers better modulated the release of KAB from the core, releasing $50 \%$ less during the first hour. The burst release of the KAU dye, observed from both coaxial and triaxial fibers, was attributed to water penetrating the porous fiber shell, allowing transient release. In another example of coaxial fiber design, water-soluble PVP was used as a core with a hydrophobic 
ethyl cellulose (EC) shell to encapsulate hydrophobic compounds of either quercetin or ketoprofen. Using this architecture, $\sim 75 \%$ of both hydrophobic encapsulants were released within $24 \mathrm{~h}$.

\section{Hydrophilic Shell—Hydrophobic or Hydrophilic Cores}

Similarly, coaxial fibers that have hydrophilic shells can facilitate the rapid release of encapsulated agents with an initial burst release of 1 to $4 \mathrm{~h}$ followed by continued transient release within $24 \mathrm{~h}$ of administration. One architecture that has been adopted to achieve rapid- or on-demand release from coaxial fibers is a hydrophilic shell in combination with a hydrophilic or hydrophobic core. In one study, zein-PVP core-shell fibers were developed that incorporated the active agent in both the core (zein) and the shell (PVP) layers ${ }^{92}$. Zein, a natural, moderately hydrophobic polymer was used to achieve immediate and transient release of the hydrophobic drug, ketoprofen. A burst release of $43 \%$ was observed within the first hour, followed by transient release of the remaining ketoprofen over $10 \mathrm{~h}$. The initial burst release was correlated with rapid dissolution of the hydrophilic shell, while the more transient $10 \mathrm{~h}$ release was attributed to the hydrophobic core. In another study, the release profile of a hydrophobic drug, asiaticoside, was compared between coaxial fibers composed of chitosan cores with either a hydrophilic alginate and PVA-blended polymer shell or a hydrophobic centella triterpenes cream shell ${ }^{93}$. The coaxial fiber with the alginate-PVA shell demonstrated $80 \%$ more asiaticoside release relative to the centella control within $10 \mathrm{~h}$, which was attributed to the shell hydrophilicity ${ }^{93}$. Additionally, the trend of burst release followed by more gradual transient release was attributed to rapid 
degradation of the alginate-PVA shell, followed by subsequent degradation of the chitosan core. While this example incorporated a polymer blend (alginate-PVA) as the hydrophilic shell, to be considered a core-shell structure, it should be noted that the material itself needs to be electrospinnable without other polymers. As this example demonstrates, hydrophilic polymers such as PVP, PVA, or PEO can be electrospun alone or in blends to create hydrophilic core and shell layers.

\section{Core-Shell Architectures with Similar Core-Shell Hydrophobicity}

Coaxial fibers comprised of both hydrophilic core and shell layers have also been investigated to provide transient release of active agents. For example, coaxial fibers fabricated with a hydrophilic PVP shell and hydrophilic cellulose acetate core were investigated. These coaxial fibers with both a hydrophilic core and shell released $31 \%$ of their hydrophobic encapsulant (epicatechin) within 10 min, followed by $80 \%$ release after $4 h^{94}$.

In addition to the utilization of materials with similar hydrophobicities, coaxial fibers consisting of identical core-shell materials have been fabricated to provide the rapid release of active agents. In one study, fibers with PVP shells and cores were investigated to provide rapid release of the hydrophobic drug, quercetin. The PVP shell-PVP core fibers released quercetin within one minute ${ }^{95}$, and this burst release was similarly observed in a separate study that used the same fiber formulation to deliver acyclovir ${ }^{96}$. In another study, the hydrophobic antibiotic, allyltriphenylphosphonium bromide, was incorporated within the core of coaxial fibers, and the volumetric ratios of core-shell solutions were varied to study 
release. Fibers comprised of zein-zein with core-shell volume ratios greater than $1: 2$ were found to suppress the burst release of the antibiotic, only releasing $15 \%$ within the first hour. In contrast, $35 \%$ and $45 \%$ of the antibiotic were released from fibers with a 1:1 core:shell volumetric ratio or blended fiber controls over the same duration $^{97}$. In a separate study, a triaxial fiber in which all three layers were comprised of ethyl cellulose provided zero-order release of ketoprofen over $20 \mathrm{~h}$ due to the gradual increase in the drug content moving from shell to the core ${ }^{98}$. These studies highlight the role of the active agent distribution within the fiber layers, suggesting that encapsulant localization within the fiber core may enhance release.

Finally, the release of fluorescently labeled bovine serum albumin (BSA) from core-shell hydrogel nanofilaments composed of a poly(lactide-co- $\varepsilon$ caprolactone) (PLCL) shell and N,N-isopropylacrylamide (NIPAAm)/N,N"methylene bisacrylamide crosslinked core was studied. The crosslinker, $N, N^{\prime}-$ methylene bisacrylamide, was used to polymerize NIPAAm during the electrospinning process. This study showed that by changing the NIPAAmcrosslinker $(w / w)$ ratio from 4:1 to $37: 1$, the release of BSA increased from 0.15 to $0.7 \mathrm{ug} / \mathrm{mg}$ over $24 \mathrm{~h}$. However, in the absence of a hydrogel within the core, BSA showed nearly complete release over the same duration. This study demonstrated that the mechanical and corresponding drug release properties could be more finely tailored by altering the NIPAAm-crosslinker $(w / w)$ ratio ${ }^{99}$.

\section{Stimuli-Responsive Coaxial Architectures}


Another method to modulate the release of active agents from coaxial fibers is to integrate stimuli-responsive layers to precisely release agents in response to surrounding physiological conditions ${ }^{100}$. Unlike stimuli-responsive uniaxial fibers, the more complex interactions between the core and shell layers in coaxial fibers can provide increased control of active agent release via $\mathrm{pH}$ - or other stimuli-based mechanisms. A variety of natural and synthetic materials have been investigated for their use in $\mathrm{pH}$-responsive applications. In one example, a coaxial fiber comprised of a lecithin-diclofenac sodium core and a Eudragit S100 shell provided the $\mathrm{pH}$-responsive release of ferulic acid for $10 \mathrm{~h}^{101}$. Ferulic acid release was facilitated under conditions of neutral $\mathrm{pH}(\mathrm{pH} 7)$, with minimal release occurring in a more acidic $(\mathrm{pH} 2)$ environment. Another $\mathrm{pH}$-sensitive polymethacrylate-based copolymer ${ }^{101-103}$, Eudragit EPO, was used to fabricate $\mathrm{pH}$-responsive antibacterial fibers. Here, Eudragit EPO cores, which dissolve below $\mathrm{pH}$ 5, were used in combination with Eudragit L100 shells, which dissolve at a pH greater than 6 . These coaxial fibers provided $\mathrm{pH}$-responsive release for an hour under slightly acidic conditions $(\mathrm{pH}$ 6) while demonstrating attenuated release in very acidic conditions $(\mathrm{pH} 2)^{104}$. Additionally, two separate studies investigated coaxial fibers comprised of Eudragit S100 shells and PEO cores to stimulate $\mathrm{pH}$-responsive release within the gastrointestinal tract ${ }^{105,106}$. In both studies, the release of hydrophobic indomethacin and hydrophilic mebeverine hydrochloride agents was minimal ( 10\%) after $2 \mathrm{~h}$ under acidic conditions, followed by rapid release for $6 \mathrm{~h}$ when switched to neutral conditions ( $\mathrm{pH}$ 7.4). Coaxial fibers comprised of cellulose acetate phthalate shells with polyurethane cores, as well as gelatin-sodium 
bicarbonate shells with PLCL cores have also been used to provide similarly rapid $\mathrm{pH}$-responsive release of ciprofloxacin and rhodamine B (Rhd B). These studies demonstrated the potential of coaxial fibers as $\mathrm{pH}$-sensitive delivery systems ${ }^{107,108}$.

Coaxial fibers with other stimuli-responsive properties have been investigated for on-demand, rapid release applications. Although studies with other stimuli-responsive systems have been limited, one study investigated the use of self-immolative polymers, or polymers that depolymerize when exposed to specific external stimuli, for rapid stimuli-responsive release ${ }^{109}$. In this study, selfimmolative fibers comprised of dibutyltin dilaurate and phenyl (4(hydroxymethyl)phenyl) carbamate were blended with polyacrylonitrile and used as shells to surround PVP cores. The fibers provided minimal release of KAB dye when incubated in water; however, the fibers depolymerized when exposed to trifluoroacetic acid, resulting in zero-order release of $\sim 40 \%$ dye within a week.

\section{Short-Term Release (One Day to One Week)}

\section{Hydrophobic Shell—Hydrophilic Core}

A key advantage of short-term release specifically for intravaginal delivery is that the burden of frequent or daily administration may decrease, thereby increasing user adherence of prophylactics and therapeutics. Traditionally, hydrophobic materials have been well-suited to provide longer durations of release (depending on the encapsulant) due to their decreased degradation rates in aqueous environments. For more traditional uniaxial hydrophobic fiber platforms, most hydrophobic small molecule drugs or larger macromolecules achieve release 
for up to one week due to the similar hydrophobic properties of the polymer and encapsulant ${ }^{17}$. This compatibility allows for hydrophobic encapsulants to partition more evenly within and distribute throughout hydrophobic polymers. However, hydrophilic agents, which have low solubility in nonpolar polymers, often partition to the fiber surface, resulting in burst release and suboptimal short-term and/or sustained-release properties. To address this challenge, coaxial fibers in which hydrophilic agents are encapsulated within a hydrophilic core and surrounded by a protective hydrophobic shell can prolong and adjust the release of hydrophilic molecules.

The use of coaxial fibers with hydrophobic shells and hydrophilic cores has been shown to extend the release of many encapsulants $82,110,111$. In one study, a coaxial fiber comprised of a hydrophobic ethyl cellulose shell with a hydrophilic PVP core was investigated for short-term release. These fibers released maraviroc over a duration of hours to days depending on the thickness of the hydrophobic shell, which was modulated via flow rate and total electrospun volume. The increased thickness of the hydrophobic shell extended encapsulant release from $24 \mathrm{~h}$ to five days by increasing the shell-to-core volume ratio from 0.5 to $4^{110}$. In another study, a PCL fiber shell surrounding a PVP-graphene oxide blended core was studied. These fibers released $65 \%$ of hydrophilic vancomycin hydrochloride within $4 \mathrm{~h}$ and attained full release of vancomycin after $96 \mathrm{~h}^{112}$. Although this coaxial fiber provided short-term release, the long-term safety of graphene oxide within the FRT is unknown, and further studies are required to assess its safety in intravaginal delivery applications. Finally, a coaxial fiber composed of a synthetic 
hydrophilic poly-cyclodextrin core and hydrophobic poly(methacrylic acid) shell reduced the burst release of a hydrophilic drug, propranolol hydrochloride, by $50 \%$, and extended release to $180 \mathrm{~h}$ relative to the 140 hour release obtained from uniaxial fibers ${ }^{113}$.

\section{Hydrophobic Shell-Hydrophobic Core}

In addition to the widely used hydrophobic shell-hydrophilic core coaxial architectures, the use of hydrophobic materials in both the core and the shell layers has also been investigated to provide the short-term release of active agents. In one study, a PCL core surrounded by an outer PCL shell was used to prolong the release of the antibiotic ampicillin. Ampicillin, a hydrophilic compound, normally localizes to the surface of PCL when spun as a uniaxial fiber, resulting in burst release $^{114}$. As an alternative, a $4 \%(w / v)$ PCL solution was used to fabricate an ultra-thin shell to delay release. In addition, the parameters for coaxial electrospinning were modified using dilute sheath solutions to improve the control of fiber diameter and morphology. The resulting coaxial fiber efficiently encapsulated ampicillin and provided short-term release for $\sim 80 \mathrm{~h}{ }^{114}$. In another study, coaxial fibers comprised of a zein shell with a PCL core reduced the burst release of the hydrophilic antibiotic, metronidazole, achieving short-term release for more than four days ${ }^{89}$.

\section{Stimuli-Responsive Coaxial Architectures}

Coaxial fibers exhibiting stimuli-responsive properties have also been investigated to provide short-term release of active agents. As one example, 
poly( $N$-isopropylacrylamide), a thermoresponsive polymer, was used as a core layer in combination with an ethyl cellulose and anhydrous ethanol shell solution. At room temperature, poly ( $N$-isopropylacrylamide) exhibits hydrophilic properties; however, at temperatures above $32^{\circ} \mathrm{C}$, the polymer demonstrates more hydrophobic characteristics. At room temperature and after $55 \mathrm{~h}$, the fibers released $65 \%$ of ketoprofen in PBS, while only $40 \%$ of the same drug was released at $37^{\circ} \mathrm{C}^{115}$.

\section{Blended Polymers in Coaxial Architectures}

Another method of prolonging release is to use blended polymers to formulate coaxial fibers, which can decrease fiber wettability. One study combined gelatin, a natural hydrophilic protein, with the hydrophobic polymer, PCL, to create coaxial fibers with increased hydrophobicity and mechanical stability relative to gelatin alone ${ }^{116}$. In one study, the release of hydrophilic doxycycline was measured from three different fiber architectures-a uniaxial PCL-gelatin blended fiber, coaxial fibers with three different cores (PCL, gelatin, or a PCL-gelatin blend) and a PCL-gelatin blended shell, and a triaxial fiber with both a PCL-gelatin blended core and outer shell and an intermediate gelatin layer. Among these five designs, uniaxial PCL-gelatin blended fibers released the most doxycycline within $24 \mathrm{~h}$ (90\%), while coaxial fibers with a PCL-gelatin core and shell released the least (50\%). Additionally, only coaxial fibers with either a PCL-gelatin or gelatin core prolonged release over five days. Furthermore, the other architectures including the uniaxial PCL-gelatin blend, coaxial fiber with PCL core, and triaxial fibers failed to release doxycycline for more than $30 \mathrm{~h}$. The burst release observed in fibers 
with PCL cores was attributed to the lack of compatibility between the hydrophobic PCL cores and hydrophilic encapsulant, which caused doxycycline to localize on the core surface. Additionally, the subsequent suboptimal encapsulant release was attributed to low water penetration into the hydrophobic core. These studies demonstrate that utilization of both hydrophobic and hydrophilic polymers alone or as blends can modulate the short-term release of hydrophilic encapsulants due to the variation in the permeability of different layers and core-encapsulant interactions.

\section{Sustained-Release (One Week to Multiple Months)}

\section{Hydrophobic Shell—Hydrophilic Core}

Similar to fibers that provide short-term release, fibers designed for sustained release commonly use hydrophobic polymers as the outer shell to prevent the fiber from undergoing rapid hydrolysis. Studies have demonstrated that the most promising coaxial architecture to achieve sustained-delivery utilizes a hydrophobic shell and hydrophilic core ${ }^{17}$. A polymer that is frequently used in coaxial fibers to provide sustained-release is poly(lactic-co-glycolic acid) (PLGA). In one study, a coaxial fiber composed of a PLGA shell was used to shield a hydrophilic core consisting of tragacanth gum. The encapsulant, tetracycline hydrochloride, served as a model hydrophilic agent. Investigators observed that PLGA (shell)-tragacanth gum (core) coaxial fibers diminished burst release and provided sustained-release of tetracycline hydrochloride for 75 days, releasing $68 \%$ of tetracycline hydrochloride during this period ${ }^{117}$. In another study, a PLGA 
(shell)-polyethylenimine (PEI, core) architecture was used to prolong the release and stability of bone morphogenetic protein-2 plasmid (pBMP2-2). The hydrophilic PEI core was used to encapsulate and retain the bioactivity of pBMP2-2, while the hydrophobic PLGA shell was used as a protective barrier to prolong release. When compared to uniaxial PLGA-PEI blended fibers, the PLGA (shell)-PEI (core) coaxial fiber exhibited both improved bioactivity and prolonged release of the pBMP2-2 plasmid. The coaxial fiber released $80 \%$ of the plasmid over 20 days, while the uniaxial fibers released the same amount over seven days ${ }^{118}$.

Polymers other than PLGA have been used as hydrophobic shells to sustain the release of active agents from coaxial fibers. One study formulated coaxial fibers containing a hydrophilic dextran core and hydrophobic PCL shell. The addition of polyethylene glycol (PEG) to the PCL shell increased the release of the encapsulated BSA by forming pores in the shell layer. Although all fibers released $\sim 20 \%$ BSA within the first $24 \mathrm{~h}$, increasing the PEG concentration increased the amount of BSA released over extended durations. Interestingly, all fibers demonstrated sustained-release regardless of PEG concentration; coaxial fibers fabricated with $5 \%$ PEG shells released $\sim 60 \%$ BSA, while fibers containing $40 \%$ PEG shells released $90 \%$ BSA over 27 days ${ }^{119}$. In another study, the relationship between PEG (core):PCL (shell) molar ratio and the release of BSA or lysozyme was investigated. The thinnest shell layers with a core:shell molar ratio of 1.59 and a core flow rate of $2 \mathrm{~mL} / \mathrm{h}$ provided complete release of both encapsulants within 24 days, compared to only $50 \%$ release from thicker fibers with a core:shell molar ratio of 0.32 and a core flow rate of $0.6 \mathrm{~mL} / \mathrm{h}$. Moreover, the fibers preserved the 
bioactivity of lysozyme and released BSA over 29 days, with no noticeable differences between BSA and lysozyme release rates $^{120}$. In addition to conventional coaxial spinning, the use of emulsion electrospinning has also been investigated to fabricate coaxial fibers, which can be electrospun using a uniaxial spinneret ${ }^{81}$. One study that used emulsion electrospinning fabricated core-shell fibers composed of a PEG-poly(D,L-lactic acid) shell and methyl cellulose core to minimize the burst release of lysozyme ${ }^{121}$. The release of lysozyme from the core was achieved over 15 days and was dependent on the percent of lysozyme loaded, while the structural integrity and bioactivity of lysozyme was protected by the shell. A later study compared these same coaxial fibers to blended uniaxial fibers composed of PCL and PEG and showed that the coaxial fibers improved sustained-release by releasing $\sim 50 \%$ of BSA over 35 days relative to blended fibers, which released $\sim 75 \% \mathrm{BSA}^{122}$.

Another study explored the effects of multiple processing parameters, including PEG and PCL concentrations, PEG molecular weight, encapsulant concentration, and fiber diameter, in modulating the release of plasmid DNA (pDNA). Plasmid DNA was encapsulated in a PEI core, and a non-viral gene delivery vector ( $r$-PEI-HA) was incorporated within a PCL shell ${ }^{123}$. An increase in fiber diameter was observed with an increase in all of the three other parameters, while the loading and release of r-PEI-HA were correlated to pDNA concentration in the fiber core and PEG molecular weight. The fibers formulated with high PEG molecular weight and low pDNA concentration exhibited $\sim 30 \%$ release of $r-\mathrm{PEI}-$ 
HA over 60 days, while the fibers with high pDNA concentration and low molecular weight PEG completely released pDNA within 60 days.

\section{Core-Shell Architectures with the Same Core-Shell Hydrophobicity}

Although coaxial architectures with similar core and shell hydrophobicities have been utilized to obtain transient and short-term release, coaxial fibers that use the same materials have been less frequently investigated to provide sustained-release. In one study, PLGA was utilized in both the core and shell layers to investigate the effect on vancomycin and ceftazidime delivery ${ }^{124}$. Both hydrophilic drugs were encapsulated within the core PLGA layer and exhibited similar burst release kinetics within the first day, followed by a second phase of more gradual release over five to ten days. Ninety percent of the antibiotics were released after 11 days, followed by complete release after 25 days, with the more gradual release attributed to the PLGA barrier layer.

\section{Applications for Intravaginal Delivery}

The enhanced tunability and versatility provided by the core and shell layers of coaxial fibers make them excellent candidates for intravaginal delivery applications. While uniaxial fibers have been studied for sustained- and stimuliresponsive release of active agents in the $F R T^{17,125-130}$, they have faced challenges in providing the sustained-release of therapeutically relevant concentrations of individual active agents and effectively modulating the release of multiple agents core ${ }^{17}$. Often, compatibility between the polymer and encapsulant can pose challenges to achieving sustained-release with uniaxial fibers, while coaxial fibers 
may circumvent this issue by integrating two different polymers, enabling the separation of agents within a compatible polymer formulation (core or shell). Moreover, the additional outer shell can help to modulate release. One can envision that with a coaxial architecture, multiple agents may be delivered against a particular infection to provide a synergistic effect or to provide protection against multiple types of viral or bacterial infections. Together, these features allow for enhanced tunability with the option of providing immediate to short-term release for on-demand applications while also providing long-term release that may be particularly useful in prophylactic or contraceptive applications.

A variety of release kinetics can be attained from coaxial fibers by using different combinations of materials in the core and shell layers. Transient or rapid release of active agents is often accomplished with the use of hydrophilic polymers due to their rapid dissolution in aqueous environments. To achieve short-term release extending to one week, a hydrophilic core in combination with a hydrophobic shell is the most frequently used architecture, enabling the slow dissolution of the shell layer, which acts as a barrier to encapsulant diffusion from the core. For sustained-release applications that require delivery on the order of weeks to months, hydrophobic polymers such as PLGA and PCL are often selected as shell polymers due to their slower degradation kinetics and biocompatibility. Yet, due to the number of parameters involved in the synthesis of coaxial fibers, two similar architectures may still be tailored to perform very differently by altering physical versus chemical properties. An example may be seen in which fibers composed of similar or even the same polymers display very 
different release rates due to the modulation of shell thickness. In these cases, thinner shells have been shown to provide more transient release, while increasing the shell thickness delays or alters the trend to more gradual release.

Coaxial fibers have been investigated previously for intravaginal delivery ${ }^{107,110}$. In one study, maraviroc release from coaxial fibers was adjusted by varying the drug loading and solution flow rates to provide release over five days ${ }^{110}$. In addition, $\mathrm{pH}$-responsive coaxial fibers have been fabricated to react in the presence of semen by utilizing the $\mathrm{pH}$-sensitive polymer cellulose acetate phthalate as a shell. The outer shell dissolved immediately after exposure to PBS, promoting $\mathrm{pH}$-responsive release of $\mathrm{Rhd} \mathrm{B}^{107}$.

Although coaxial fibers have shown promise in general drug and initial intravaginal delivery applications, further refinements are required to expand their overall utility. First, compatibility between the solvents of the two polymer electrospinning solutions may limit the potential combinations of core-shell materials and encapsulated agents to achieve successful electrospinning. Additionally, residual solvents from the electrospinning process may interact with and inactivate encapsulated active agents in the core layer. Therefore, while research in coaxial fiber design is still ongoing, other fiber architectures such as multilayered fibers may offer additional advantages to advance intravaginal delivery.

\section{Multilayered Electrospun Fibers}

\section{Multilayered Fiber Architectures and Properties}


Multilayered fibers can provide layer-by-layer delivery platforms that are relatively simple and inexpensive to fabricate while allowing for the encapsulation of different active agents within the individual layers. The topology, thickness, and composition of each individual layer can be easily tuned to provide different release properties based on the envisioned application. Moreover, multilayered fibers have been shown to have increased mechanical stability and flexibility compared to coaxial fibers ${ }^{131}$. While the interactions between two or more polymer solution interfaces must be considered for coaxial fibers, multilayered fibers can be fabricated from normally incompatible polymers due to their sequential versus simultaneous fabrication process.

Electrospun multilayered fibers can be fabricated by sequential layering, stacking, or interweaving fibers ${ }^{132-134}$. In sequential layering, the first layer of polymer is electrospun onto a collector, followed by electrospinning additional polymer layers directly onto the same collector. In comparison, "stacking" fibers refers to individually electrospinning each layer separately and subsequently adhering individual layers together post-spin. Stacked fibers share similar physical properties with sequentially-layered fibers, enabling temporally-programmed or spatially-specific delivery of active agents ${ }^{135}$. Finally, the fabrication of interwoven fibers utilizes dual or multiple-syringes to simultaneously electrospin two or more different polymer solutions (usually one hydrophilic and hydrophobic) onto the same collector. In contrast to fibers produced using the sequential layering and stacking processes, which have distinct, separate layers of polymeric fibers, interwoven fibers result from the blending of these different polymer solutions from 
syringes placed opposite of or adjacent to each other into one integrated layer ${ }^{136-}$ 138. This technique seamlessly integrates both hydrophilic and hydrophobic polymers in a way that prevents unwanted interactions between the electrospun polymer solutions ${ }^{138,139}$ while enabling the porosity of the hydrophilic fibers to be altered to more finely tune fiber degradation ${ }^{140}$. Although interwoven fibers do not have a shell layer, the interwoven architecture has been beneficial in promoting cell adhesion and growth and has the potential to more finely modulate active agent release via porosity-based mechanisms for drug delivery applications ${ }^{141,142}$.

Regardless of fabrication technique, multilayered fibers are beneficial in that they can temporally modulate the release of multiple agents from a single delivery platform and can provide additional tunability by modulating the barrier or discrete layers of the multilayered structure (Figure 1.4). In addition, the ability to impart spatially-specific release-where specific layers of the multilayered fiber possess distinct release profiles-is a key advantage of this architecture. This advantage may be envisioned for intravaginal delivery applications where one layer provides rapid active agent release to the mucus while another layer enables sustaineddelivery specific to underlying epithelial or immune cells ${ }^{132,134}$. For interwoven multilayered fibers, studies have shown that the incorporation of a hydrophilic polymer can alter the overall porosity and wettability ${ }^{140,143,144}$, while using a hydrophobic outer layer in multilayered fibers (similar to coaxial fibers) can decrease surface wettability and corresponding active agent release ${ }^{145}$. 
A

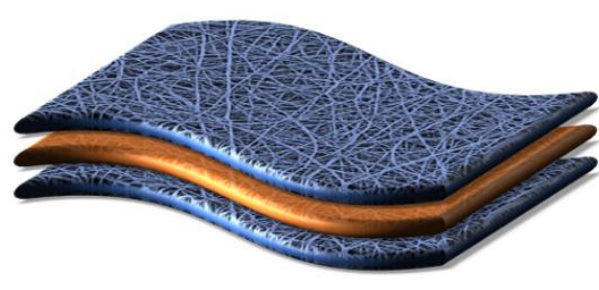

B

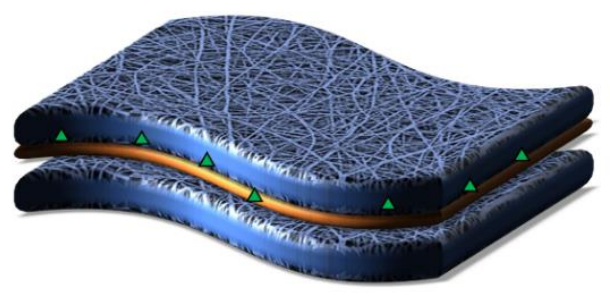

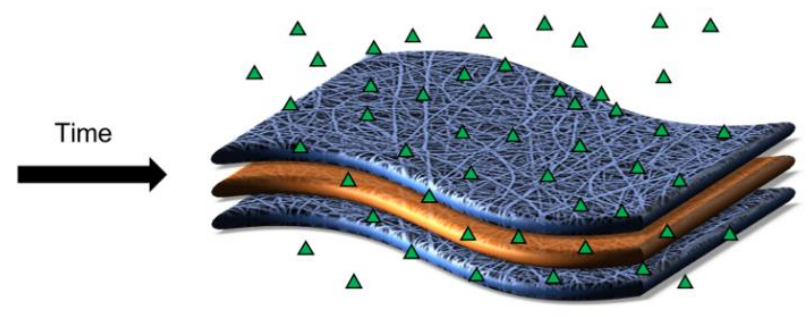

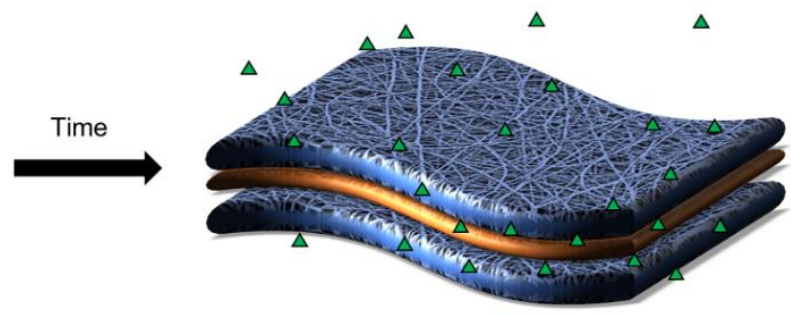

Figure 1.4. Schematic of anticipated active agent release from multilayered fibers. One method to modulate the release of active agents (shown in green) is to vary the thickness of the outer layer (shown in blue). (A) A thin outer layer provides both rapid burst release and limited sustained-release of encapsulants. (B) In contrast, increased outer layer thickness can delay the release of some active agents.

While the process of creating multilayered fibers is well established, more work is required to elucidate how each polymer layer impacts release kinetics. Physical properties including the pore size, fiber diameter, and thickness of traditional uniaxial fibers are known to impact the delivery kinetics of active agents from individual layers. Thus, the presence of one or more fiber layers can contribute to the complexity in establishing and predicting the release kinetics of diverse active agents from differently layered architectures. Despite these considerations and complexities, the adoption of different layering techniques to create multilayered fibers can achieve diverse patterns of release for transient, short-term, and sustained-release applications. 


\section{Release Kinetics from Multilayered Fibers}

\section{Transient and Short-Term Release}

Multilayered fibers have shown promise in providing transient and shortterm release of active agents. Conventionally, a hydrophilic layer serves as a reservoir for active agents, while hydrophobic materials provide an outer shell layer to prolong release. One study utilized a multilayered fabrication approach to encapsulate the hydrophobic antibiotic, gentamicin, in a hydrophilic PVA center layer and utilized a PU outer layer to envelop the inner PVA fiber ${ }^{146}$. Three separate fibers were fabricated by altering the thickness of the PU outer layer between 3.4 and $8.1 \mu \mathrm{m}$. The release of gentamicin was modulated with the thinnest PU layer $(3.4 \mu \mathrm{m})$ demonstrating complete release within $1 \mathrm{~h}$, relative to $10 \%$ release obtained from the thickest layer $(8.1 \mu \mathrm{m})$. Furthermore, the thickest PU layer continued to release gentamicin for $24 \mathrm{~h}$. Another study using interwoven electrospun fibers containing PEO and PCL demonstrated that by adjusting the ratio of the two polymers, tunable fiber degradation could be achieved from the

resulting changes in pore size and porosity ${ }^{138}$. Although this study investigated interwoven fibers to enhance cell infiltration through the pores, the use of sacrificial fiber layers may be applied to modulate active agent release from the fibers for intravaginal delivery applications ${ }^{138}$.

In addition to modulating the outer layer thickness and overall fiber composition, alterations to the number of layers have been shown to impact active agent release. In one study, fibroin-gelatin blended uniaxial fibers exhibited release 
of trypan blue, fluorescein isothiocyanate (FITC)-inulin, and FITC-BSA within minutes ${ }^{147}$. In contrast, multilayered fibers composed of the same materials extended the release of all three model compounds to 28 days ${ }^{147}$. In another study, dual-release, multilayered electrospun fibers containing the model dyes, 5,10,15,20-tetraphenyl-21H,23H-porphinetetrasulfonic acid disulfuric acid (TPPS) and chromazurol B, were encapsulated in four-layered PLCL (75:25) fibers. The release rate and duration of the dyes were controlled by the fiber diameter and individual fiber layer thicknesses. Minimal release of both dyes was observed for the first $15 \mathrm{~min}$, followed by a quasi-linear release profile for up to $4 \mathrm{~h}$. However, increasing the thickness of dye-loaded layers resulted in higher quasi-linear release rates due to the reduced density of the fiber surface ${ }^{148}$. In another study, the transient release of ketoprofen was achieved using trilayer fibers composed of two EC outer layers surrounding a center PVP fiber. These fibers provided nearly complete release of ketoprofen within $24 \mathrm{~h}^{132}$. Last, asymmetric multilayered polylactide fibers with different designs on each side were fabricated to prevent liver cancer recurrence by promoting one-sided prolonged chemotherapeutic release $^{149}$. The fiber was composed of five poly(lactic acid) (PLA) layers, with each layer serving as either a barrier to release or a drug encapsulating reservoir. In vivo studies in a murine model demonstrated tumor suppression for at least four days, indicating that the multilayered fiber may provide localized chemotherapy for short-term durations ${ }^{149}$.

Multilayered fibers with stimuli-responsive properties have also been investigated for transient and short-term release applications. In one of the first 
studies to investigate multilayered architectures, the $\mathrm{pH}$-responsive polymers, poly(acrylic acid) (PAA) and poly(allylamine hydrochloride) (PAH), were electrospun together to create a blended fiber. These fibers were loaded with a low molecular weight cationic molecule, methylene blue, and demonstrated rapid release of methylene blue ( 10 $\mathrm{min})$ at a neutral $\mathrm{pH}(7.4)$. However, by gradually adjusting the $\mathrm{pH}$ from 6 to 2 in aqueous solutions, the step-wise $\mathrm{pH}$-responsive release of methylene blue was achieved over three and a half days. Building upon this work, the effect of coating the fibers with a thermoresponsive polymer blend, poly( $N$-isopropylacrylamide)-PAA, or perfluorosilane was assessed. The addition of the thermoresponsive poly( $N$-isopropylacrylamide)-PAA coating modulated methylene blue release via temperature. Above a critical temperature, the thermoresponsive polymer became insoluble and formed intramolecular hydrogen bonds, which led to the release of methylene blue within 50 min (PBS, pH 7.4). In comparison, coating with perfluorosilane modulated release for up to $20 \mathrm{~h}$ at neutral $\mathrm{pH}$. When both the $\mathrm{pH}$-responsive and multiple layers of thermoresponsive polymers were integrated and evaluated at 25 and $40^{\circ} \mathrm{C}$, dye released for a maximum of $10 \mathrm{~h}$ regardless of layer thickness ${ }^{150}$.

\section{Sustained-Release}

The ability of multilayered fibers to provide long-term release has been demonstrated in a variety of studies ${ }^{77,78,151}$. In one study, the release of a hydrophobic chemotherapeutic agent, 7-ethyl-10-hydroxycamptothecin (SN-38), was prolonged to 30 days by using a triple-layered fiber in which $\mathrm{SN}-38$ was encapsulated in the center layer and surrounded by two superhydrophobic outer 
layers consisting of PCL and poly (glycerol monostearate-co- $\varepsilon$-caprolactone) ${ }^{145}$. Similar to the trends seen for transient and short-term release from multilayered fibers, increasing the thickness of the outer fiber substantially improved the longevity and amount of drug released. In another study, multilayered fibers comprised of a PCL shell and a PEO/Rhd B core were fabricated to assess the effect of increasing the outer layer thicknesses between $46.1,68.9$, and $186.1 \mu \mathrm{m}$

152. While the thinnest $46 \mu \mathrm{m}$ layers released $85 \%$ of Rhd B in one day, the 68.9 and $186.1 \mu \mathrm{m}$ layers increased release to 15 and 25 days, respectively. Moreover, the release from the two fibers with the thicker outer layers demonstrated zeroorder kinetics, producing gradual, even release of drug with respect to time.

\section{Applications for Intravaginal Delivery}

Multilayered fibers have shown promise as a platform to co-deliver or prolong the release of active agents in different environments. The process of creating multilayered fibers is relatively simple, eliminating the more complex setup and considerations of polymer-solvent interactions between the adjacent, simultaneously spun layers present in coaxial spinning. By removing this complexity of interactions, multilayered fibers can achieve "programmed release" by simply modulating the thickness of each layer.

Multilayered fibers possess other unique features that make them excellent candidates for intravaginal delivery applications. One of the unique strengths of multilayered fibers is that they can provide spatially-specific release in that, unlike other architectures, the individual layers of multilayered fibers can be designed for 
specific and discrete purposes. For example, one layer may be designed to improve mucoadhesion for enhanced longevity and biocompatibility within the FRT, while another layer may provide release of active agents dependent on its location within the multilayered matrix. Compared to coaxial fibers, the optimization of multilayered fibers is not limited by solvent compatibility, as they can be sequentially spun and assembled post-fabrication. Moreover, multiple individually spun layers can increase the ease of encapsulating multiple types of active agents, which serve mechanistically different roles against a single type of viral infection or as a multipurpose viral-contraceptive or viral-bacterial dosage form. Finally, each fiber layer can be adjusted to have distinct mechanical properties that include tensile strength, porosity, and elasticity, important for comfort and user preference ${ }^{153}$.

To date, the use of multilayered fibers for intravaginal delivery has been briefly explored $67,110,134$. In one study, circular sheets of pre-spun PVP and PVPEC fibers were stacked and annealed via a pressed metal die that was dipped in solvent. The die annealed the edges of the stacked fibers, creating a multilayered fiber with a PVP inner layer surrounded by blended PVP-EC sheaths. Other multilayered fibers were also constructed by folding the outer layers and pressing the seams. Both types of multilayered fibers encapsulated the hydrophilic compound maraviroc and provided biphasic release, exhibiting an initial burst release followed by short-term release for up to five days. Another study from the same group examined tenofovir (TFV) localization within stacked PCL/PLGA fibers. It was found that TFV localization within the multilayered fiber could be 
predicted by considering the changes in polymer crystalline structure caused by encapsulant-polymer interactions and correlating drug-polymer hydrophilicity ${ }^{67}$.

Both multilayered and coaxial fibers have the potential to provide tunable and sustained-release; however, each architecture still faces the challenges surrounding FRT delivery. For example, the interplay between two polymer solutions still needs to be considered for interwoven multilayered (and coaxial) fibers, which may result in challenges to altering active agent release. Additionally, as stated previously, the most significant obstacles to intravaginal delivery are providing a dosage form that can facilitate active agent penetration of mucus and retention and release of therapeutically relevant agent concentrations within the FRT. To improve retention, fibers can be fabricated using polymers or polymer blends that have mucoadhesive properties. However, this longevity is rarely translated to active agents once they have been released from fibers. Thus, new measures may be considered to provide efficacious and sustained-delivery from fibers.

\section{Composite Nanoparticle-Fiber Delivery Vehicles}

\section{Nanoparticle-Fiber Architectures and Properties}

Over the past two decades, polymeric NPs have been extensively studied as efficacious drug delivery platforms for a variety of applications. Polymeric nanoparticles are an attractive option for intravaginal delivery relative to traditional delivery platforms such as gels and films due to the tunability of active agent release, ability for surface modification, potential for targeted delivery, enhanced 
distribution potential, and the often resulting enhanced efficacy of encapsulated agents. Additionally, polymeric NPs have been shown to elicit minimal immune response and to improve the delivery and bioactivity of biologics ${ }^{40,154,155}$. Although metallic nanoparticles have also been explored for use in many drug delivery applications, they have been less commonly administered within the FRT, hence, a more comprehensive review of their applications may be found in ${ }^{156,157}$.

Many physicochemical characteristics of NPs can be altered, such as particle size, surface charge, and hydrophobicity, which contribute to their success in achieving sustained-release and localization to target sites ${ }^{158}$. Although NPs have proven to be effective delivery platforms, as discussed in previous reviews ${ }^{159,160}$, achieving the prolonged release of active agents can be difficult due to the natural clearance mechanisms of the FRT. In particular, NPs are challenged with retention in the vaginal cavity due to mucus clearance and transport through mucus to underlying tissue ${ }^{39,161,162}$. These challenges may be overcome by incorporating NPs into electrospun fibers, thereby creating a composite delivery vehicle that complements the capabilities of both technologies. One might envision that fibers may act as a reservoir for NPs, improving NP and active agent retention, while the innate fiber porosity can help to more finely tune encapsulant release from NPs relative to the release observed from freely administered NPs or fibers.

Nanoparticle-fiber composites are dual-component systems that have the ability to alter the release kinetics of active agents from NPs or NPs themselves ${ }^{163,164}$. Often, the active agent of interest is encapsulated within the NPs, which are then preloaded into polymer solutions for subsequent 
electrospinning. While a variety of inorganic NPs have been incorporated into fibers $^{165-167}$, concerns still persist regarding the safety of their use relative to polymeric NPs, particularly for intravaginal applications. By utilizing biocompatible polymeric materials for both nanoparticles and fibers, composites may provide safe and prolonged release for clinical applications.

\section{Release Kinetics from Nanoparticle-Fiber Composites}

\section{Transient Release}

Nanoparticle-fiber composites have been used to rapidly release NPs and their encapsulated agents. A study was conducted with hydrophilic PVA and PEO fibers that incorporated PLGA NPs that contained the dye, Coumarin $6^{168}$. PEO fibers released $90 \%$ of NPs within 30 min when immersed in a 50:50 ethanol:PBS solution, followed by additional release $(5 \%)$ after $3.5 \mathrm{~h}$. In comparison, PVA fibers released approximately $70 \%$ of PLGA NPs within 30 min, followed by a decrease in NP release (15\%) over $8 \mathrm{~h}$. Slightly slower release over $24 \mathrm{~h}$ was observed when PVA fibers were crosslinked prior to NP incorporation. This study highlights that nanoparticle-fiber composites can be used to successfully incorporate NPs and to modulate the transient release of NPs from these composites within aqueous solutions $^{168}$.

\section{Short-Term Release}

Several studies have utilized nanoparticle-fiber composites to provide the short-term release of active agents. One group explored a composite drug delivery system that encapsulated the antibiotic, erythromycin, in gelatin NPs and free 
lidocaine hydrochloride within PVA-chitosan blended fibers ${ }^{169}$. Eighty percent of the lidocaine hydrochloride was released from the fibers within $54 \mathrm{~h}$, while $70 \%$ of the erythromycin was released after $70 \mathrm{~h}$. In contrast, free gelatin NPs released $90 \%$ of erythromycin within the same duration. In a separate study, chitosan-PEO blended fibers containing methoxypolyethylene glycol (mPEG)-b-PLA micelles demonstrated a low initial burst release (15\%) of 5-fluorouracil (5-FU), followed by prolonged release $(91 \%)$ for $109 \mathrm{~h}^{170}$. In another study, the release of free hydrophobic naproxen and chitosan nanoparticles containing Rhd B was studied from PCL fiber scaffolds ${ }^{171}$. Rhodamine B exhibited low levels $(5 \%)$ of burst release, while $30-40 \%$ of naproxen was released within the first $2 \mathrm{~h}$. Moreover, after 72 h, only $20 \%$ of Rhd B was released, while $60 \%$ of naproxen was released. The rapid release of naproxen was achieved via incorporation within the fiber scaffold, while the extended release of Rhd B was obtained and enhanced through nanoparticle-fiber encapsulation. These results demonstrate the utility of nanoparticle-fiber composites in providing the short-term release of multiple agents.

\section{Sustained-Release}

Nanoparticle-fiber composites have also demonstrated long-term release capabilities in several studies. In one study, dual-release nanoparticle-fiber composites were used to mend and treat critically sized calvarial defects in rats ${ }^{172}$. These composites, consisting of PCL-Co-PEG fibers encapsulating dexamethasone and BSA NPs and loaded with bone morphogenic protein-2 (BMP2), demonstrated sustained-release of both molecules over 35 days. Another study 
explored the incorporation of siRNA into chitosan NPs and PLGA fiber composites $^{164}$. In these composites, the release of active siRNA was sustained in vitro, with $95 \%$ of siRNA released from the fibers over 32 days, while gene silencing activity was maintained. Sustained-release from nanoparticle-fiber composites was also demonstrated in another study with chitosan-PEO electrospun fibers that were loaded with PLGA NPs encapsulating phenytoin. Nearly complete release of phenytoin from the composite scaffold was achieved over nine days ${ }^{173}$. Lastly, PLA fibers encapsulating chitosan particles provided sustained-release of BSA (45\%) for 27 days, while chitosan particles alone released $80 \%$ BSA in 14 days $^{174}$.

In addition to NP incorporation within traditional uniaxial or blended fibers, NPs have been incorporated in more complex fiber architectures to prolong the release of active agents. For instance, the effect of combining a multilayered fiber architecture with nanoparticle-fiber composites was investigated by fabricating alternating layers of poly-L-lactic acid (PLLA) and PCL fibers with layers of PCL fibers encapsulating positively-charged chitosan BSA NPs ${ }^{175}$. The multilayered composite released $80 \%$ of the BSA in approximately eight days, whereas the monolayer control released the same concentration of BSA within $24 \mathrm{~h}$.

\section{Applications for Intravaginal Delivery}

Composite delivery vehicles containing nanoparticles and fibers have thus far been primarily studied in wound healing and tissue engineering to fabricate scaffolds for tissue regeneration and bone remodeling $97,176-178$. However, these 
platforms may be promising candidates for intravaginal delivery applications due to their structural stability and ability to sustain the release of active agents. In such systems, the fibers may be utilized as a reservoir for NPs to aid in intravaginal retention by helping to decrease NP clearance during shedding. In addition, it is envisioned that, depending on fiber formulation and, importantly, NP size and charge, NP (and active agent) release may be modulated, enabling NPs to traverse mucus and deliver agents to target cells that reside in the epithelium or underlying lamina propria. Similar to other architectures, fiber parameters such as polymer composition and size can be tailored to impact release in combination with altering NP composition, size, and loading within the fiber.

For intravaginal delivery applications, NPs can impart cell specificity, cell internalization, as well as mucoadhesive or mucopenetrative properties to their encapsulated active agents ${ }^{25}$. Numerous studies have demonstrated the ability of NPs to enhance cell targeting via surface modification ${ }^{179,180}$. Additionally, surface modification can increase cell internalization, which may enhance the transport, subcellular localization, and corresponding efficacy of drugs like tenofovir disoproxil fumarate (TDF), which require cell internalization. Furthermore, the NP surface charge can be modulated to provide either mucoadhesive or mucopenetrative properties that further enhance active agent delivery. Additionally, fibers can be fabricated to encapsulate NPs for sustained-release as well as free agents for rapid release, providing both on-demand and sustainedrelease in one platform. Finally, nanoparticle-fiber composites, when coupled with 
coaxial or multilayered fiber architectures, provide an attractive strategy to retain and sustain the release of active agents within the FRT (Figure 1.5).

A
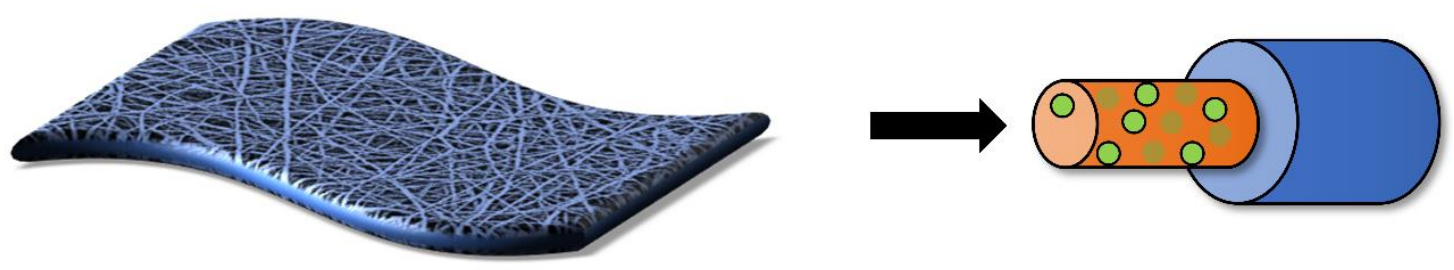

B
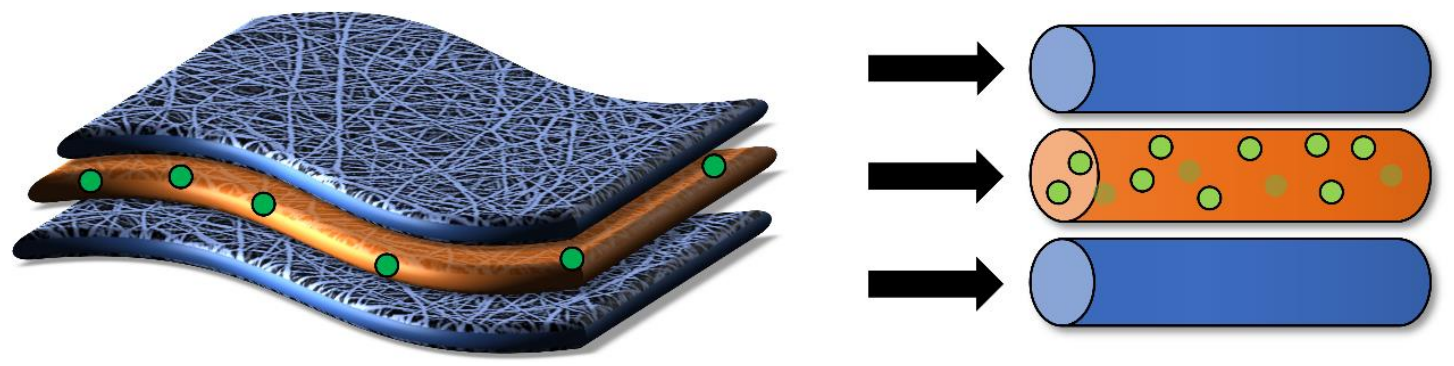

Figure 1.5. Schematic of electrospun nanoparticle-fiber composites that integrate coaxial and multilayered fiber architectures. (A) Coaxial fibers can be fabricated to encapsulate nanoparticles (NPs) within the core fiber, conferring sustained- or delayed-release of active agents that are encapsulated in NPs (shown in green). (B) Multilayered fibers that encapsulate NPs can also act as reservoirs for either NP or active agent release.

As with multilayered fibers, the use of nanoparticle-fiber composites has only recently been investigated for intravaginal delivery. In a proof-of-concept 
study, rapid-release PEO, PVA, or PVP fibers encapsulated PLGA NPs containing C6 dye or etravirine drug ${ }^{34}$. In this study, composites and free NPs were administered within murine FRTs and assessed for retention and release. The encapsulated nanoparticles exhibited a 30 -fold increase in retention in the mouse FRTs relative to free NPs. Furthermore, nanoparticles alone provided transient release of etravirine, while all nanoparticle-fiber composites demonstrated release for up to seven days. To date, this is the only investigation of nanoparticle-fiber composites for use in intravaginal delivery. However, the significant difference in retention and release rate achieved with nanoparticle-fiber composites highlights the immense potential of this architecture for sustained-delivery in the FRT.

Although combining nanoparticles and electrospun fibers into one delivery vehicle has demonstrated potential, challenges exist for this platform. The major concern is related to the concentration of nanoparticles that can be effectively encapsulated within fibers without hindering the ability of the polymer solution to be electrospun ${ }^{181}$. Furthermore, the concentration of active agent may decrease with the use of a coaxial or multilayered architecture, as only specific layers of the fiber will encapsulate NPs. Finally, polymeric NPs are often comprised of the same or similar polymers as electrospun fibers, thus care must be taken to prevent polymer solvents from dissolving the NPs prior to or during the electrospinning process ${ }^{182}$. Moreover, the morphology of NPs may also be adversely affected by electrospinning voltage. These factors limit the combinations of fiber and nanoparticle materials available for composite fabrication. Thus, for composite 
delivery applications to succeed, polymer choice and electrospinning conditions must be taken into consideration.

\section{Future Directions and Discussion}

Within the past decade, electrospun fibers have been explored as a multipurpose delivery platform to prevent and treat sexually transmitted infections (STIs). For intravaginal applications, fibers have typically been uniaxially electrospun to release active agents targeted to HIV-1/HSV-2 infections and contraceptive applications. However, other electrospun architectures have been developed that may provide more finely-tuned active agent release, the encapsulation of multiple agents, and longer release durations, desirable for nextgeneration vehicles. Given this, the goal of this review was to summarize the advancements in electrospun fiber architectures including coaxial, multilayered, and nanoparticle-fiber composites, to meet these needs, and to review their use in other drug delivery applications. We sought to relate different temporal regimens of delivery, including transient (occurring within hours), short-term (spanning hours to one week), and sustained (extending from one week to months), to architectural design and materials selection to help guide the design of future platforms that meet the unique temporal needs of intravaginal delivery.

One of the major challenges facing intravaginal delivery is the lack of user adherence surrounding the administration of current delivery platforms. Several clinical trials have highlighted how a lack of user adherence contributes to decreased efficacy in clinical trials. In both the FACTS-001 and VOICE trials, South 
African women deemed high risk for HIV-1 exposure were given antiretroviral TFV gels to administer prior to intercourse ${ }^{104,183}$. Despite the known efficaciousness of TFV, the gels provided suboptimal protection against HIV-1 infection, which was attributed to low user adherence of the gels prior to intercourse. Another study examined the efficacy of gels that incorporated the antiviral polysaccharide, carrageenan, in women in Thailand. This study demonstrated similarly disappointing clinical outcomes, with low user adherence considered the most significant reason for the lack of clinical efficacy ${ }^{184}$. Negative outcomes in other trials such as PRO-2000 and cellulose sulfate gel studies, which examined the efficacy of anti-HIV gels in female populations, further validated these studies, highlighting that both user preference and adherence regimens must be considered during product design rather than at the clinical trial stage. As a result of these studies, there has been an increased emphasis to design vehicles that decrease the administration frequency by prolonging active agent release after a single topical application.

In conjunction with improving user adherence, the development of multipurpose delivery vehicles that offer long-term protection against the various stages of a single infection or a diversity of different types of infections is highly desirable ${ }^{185}$. For single infections, a delivery platform may administer multiple agents with different mechanisms of action that target different stages of the viral or bacterial life cycle. However, the increased likelihood of viral co-infections, such as HSV-2 and HIV, as well as bacterial and fungal infections will likely require coadministration of antiviral and antimicrobial agents to be successful. Furthermore, 
applications that seek to meet both antiviral and contraceptive needs in the same dosage form will require the incorporation of multiple types of agents to expand a platform's effectiveness. Therefore, a delivery platform that has the capability to release multiple active agents, each over time frames relevant to the application or active agent, will have greater utility and enable more convenient administration schedules based on specific user needs.

Despite these needs, tailoring the delivery of multiple types of active agents for viral, bacterial, fungal, and contraceptive applications is an ambitious goal given the unique chemical properties of each agent. For example, the antiretroviral TFV and its pro-drug TDF have similar structures and both work as nucleoside reverse transcriptase inhibitors yet possess markedly different hydrophobicities. As such, a delivery platform designed to prolong TFV release may result in different release kinetics of TDF, requiring the formulation of distinct delivery vehicles specific to the selected active agents $67,127,129,186,187$. Furthermore, each active agent may necessitate specific temporal dosing regimens to provide protection or treatment. For example, it may be desirable to administer viral entry inhibitors, which inactivate virions prior to cell entry, over a different time frame than active agents that work inside of cells and need to transport through and localize to target tissue. Several studies have investigated this and have found that more complex and specialized architectures may be useful to achieve temporal delivery goals by tuning the release properties of multiple encapsulants for multiple targets ${ }^{188,189}$. Similarly, for contraceptive applications, on-demand and/or zero-order release with equivalent daily dosing may be desirable for spermicides and hormonal/non- 
hormonal contraceptives, respectively. Conversely, it may be desirable to deliver active agents such as hormones and small hydrophilic drugs (e.g., etonogestrel and acyclovir) within the same time frame for simultaneous long-term contraception and prevention. However, the drastically different chemical properties of these agents will require more complex solutions to achieve similar release profiles. Given this, multipurpose intravaginal delivery platforms must be tailored to maximize the efficacy of individual active agents, including small molecule drugs, proteins, antibiotics, hormones, and live organisms (e.g., probiotics), to meet the needs of these diverse applications.

While providing distinct release profiles of different active agents is an important criterion for the development of future intravaginal platforms, to date, intravaginal rings (IVRs) are the only platforms that provide delivery over a duration of weeks to months ${ }^{190-194}$. Furthermore, IVR studies indicate that more complex dosage forms, such as rings with drug-encapsulating pods, may more likely succeed, particularly in challenging delivery scenarios, e.g., achieving the sustained-release of small hydrophilic molecules ${ }^{188}$. These and other studies $^{188,189,195,196}$ emphasize the need to offer alternative delivery vehicles for women, with the key lesson that platform architecture must be designed to consider the hydrophobicity and chemical compatibility of the encapsulants in combination with its surrounding materials.

In addition to the development of fibers with more complex architectures, active agent release and transport from these platforms must be assessed. Tissue mimetics and ex vivo tissues have been used to assess these parameters within 
the context of intravaginal delivery applications ${ }^{127,197-202}$. One of the most common ways in which to assess intravaginal delivery is by using human ectocervical tissue explants derived from patients ${ }^{198-202}$. These explants provide a representative environment in which to measure transport by accounting for the three-dimensional structure of patient tissue. However, patient-specific variations and tissue availability can limit the use of vaginal explants. Given this, organotypic threedimensional vaginal tissue models such as Epivaginal ${ }^{\mathrm{TM}}$ tissue have been created to help evaluate the safety, transport, and efficacy of active agents within an FRT mimetic 203,204 . Other in vitro models have also been developed to explore bacteria and host cell interactions in the reproductive environment ${ }^{205}$. Moreover, within the past decade, new biomarkers and assay endpoints have been identified and studied in different models to more fully assess microbicide interactions with the $\mathrm{FRT}^{206}$. The use of tissue models promises to streamline the assessment of future fiber platforms as viable intravaginal delivery platforms.

To date, a variety of studies have developed uniaxial electrospun fibers for intravaginal applications, including HIV prevention ${ }^{67,126-130,186,187,207-210}$. In these studies, electrospun fibers have demonstrated promising potential for intravaginal applications due to their mucoadhesive characteristics, mechanical properties, and ability to be fabricated in different shapes and sizes $^{64}$. Depending upon the polymer hydrophilicity, traditional uniaxial fibers have been formulated as transient, shortterm, or long-term delivery platforms. For the purposes of on-demand and shortterm release, many of these studies use hydrophilic fibers, which dissolve or degrade quickly. In contrast, fibers consisting of more hydrophobic materials are 
expected to persist within the FRT, acting as reservoirs to sustain the release of active agents. We envision (and have observed) that long-term delivery vehicles maintain their structure during the delivery duration of interest and may require physical removal from the FRT, similar to current IVRs. However, one of the key challenges for intravaginal delivery has been to sustain the release of small hydrophilic antiretrovirals due to their rapid diffusion through the porous fiber matrix, solubility in aqueous solutions, and chemical incompatibility with hydrophobic polymer cores ${ }^{17}$. Many of these uniaxial fibers demonstrated burst release of hydrophilic agents followed by short-term release ${ }^{70,211}$, partially attributed to the localization of hydrophilic agents on the fiber surface. Compounding this, concerns exist that the subsequent release of active agents may be insufficient to provide complete protection against future infections. While blended uniaxial fibers have been moderately successful in addressing these challenges, more work is required ${ }^{130}$.

The primary parameters that impact release from uniaxial fibers are the choice of solvent and polymer. Other factors such as polymer concentration and electrospinning parameters also play a role in attaining different release profiles; however, it is unlikely that these factors alone are sufficient to overcome the challenge of delivering sustained and therapeutically-relevant concentrations of hydrophilic agents. Furthermore, it is difficult to utilize traditional uniaxial fibers for the encapsulation of multiple diverse agents such as large proteins and small drugs. Due to these issues, other electrospinning architectures may be better suited to meet the diverse challenges of intravaginal delivery. 
As discussed previously, coaxial fibers have shown promise for the encapsulation and release of small hydrophilic and hydrophobic molecules, which may be useful for intravaginal delivery applications. The different goals of transient, short-term and long-term release can be achieved by changing the composition and hydrophobicity of core and shell materials as well as by modulating the shell thickness and core:shell ratio. As described, the shell layer can help regulate active agent release, while the core layer is designed to provide optimal compatibility with an encapsulant. For instance, by using $\mathrm{pH}$-responsive polymer shells, an immediate stimuli-responsive release of agents can be achieved when the fiber is in contact with semen. In this scenario, the core layer may be tailored to encapsulate multiple agents, while the shell, comprised of $\mathrm{pH}$-sensitive polymers, retains encapsulants until needed. Another advantage of coaxial fibers is that they can be fabricated to exploit drug-polymer hydrophilicities. For example, a coaxial fiber comprised of a hydrophobic shell and hydrophilic core can be utilized to provide long-term release of hydrophilic compounds. Agent encapsulation into both layers would allow for both transient burst release from the shell due to surface localization and high loading and sustained-release from the core layer. Finally, coaxial fibers can provide release of biological agents such as large proteins. Coaxial cores may be engineered to achieve high protein encapsulation and biocompatibility, while shells can be constructed with porous surfaces, allowing tunable release. This is particularly significant given that many biologics are being investigated as future viral prophylaxes and therapeutics. Although coaxial electrospinning is a more complex process that requires additional 
optimization, relative to uniaxial spinning, it may enable a versatile platform for transient, short-term, and long-term release ${ }^{130}$.

Multilayered fibers combine different polymer layers via sequential or postspinning to incorporate multiple and chemically distinct drugs within specific layers, thereby tailoring the release kinetics for each encapsulated agent. Multilayered interwoven fibers can be utilized to provide transient release using sacrificial layers to encapsulate agents for on-demand applications. The sacrificial layers comprised of hydrophilic polymers would provide on-demand release of agents based on their immediate degradation when exposed to physiological fluids. Active agent release can be further modulated by the number, thickness, and porosity of each fiber layer ${ }^{212}$. Moreover, blank fibers may be incorporated within the multilayers to either act as a physical barrier for sustained-release or for contraceptive purposes. The layer thickness and level of porosity of blank fibers can be conveniently modulated to delay the release of small hydrophilic molecules from the drug-loaded layers, serving to prolong release. Additionally, multilayered fibers have the potential to deliver biologics and non-hormonal contraceptives. These agents, although efficacious, may degrade when exposed to harsh solvents during the electrospinning process. By incorporating these active agents in distinct layers and integrating barrier layers, multilayered fibers can provide long-term release of drugs and biologics while retaining their activities.

While each of these strategies offers advantages relative to uniaxial spinning, the delivery of active agents may be further enhanced by integrating nanoparticles with fibers. A composite platform may offer a new alternative to 
address the challenges of intravaginal delivery, such as the maintaining active agent stability, providing cell-specific targeting (via NPs), and enhancing cell internalization. Like electrospun fibers, nanoparticles can be designed to encapsulate virtually any compound. The limitations of nanoparticle-fiber composites mentioned earlier may be overcome by utilizing fibers as a reservoir for both active agents and nanoparticles to release multiple therapeutics. Furthermore, the release rates of encapsulants from both nanoparticles and fibers may be modulated by adjusting the composition of the polymeric scaffold. For ondemand transient release, hydrophilic polymers may be used to enable rapid release of NPs for immediate distribution through and enhanced retention within tissue. In contrast, more hydrophobic fibers may be used to delay the release of NPs or NP-encapsulated agents. Although drug-polymer hydrophobicity is a major contributor to release, other factors such as polymer choice, molecular weight, and crystallinity, as well as solvent choice and electrospinning parameters, also affect the release of agents from fibers.

The application of advanced fiber architectures has only recently been explored in the context of intravaginal delivery. Advanced fiber architectures demonstrate the potential to provide the sustained-release of individual active agents in addition to concurrently providing both transient and sustained-delivery of multiple active agents. These are key advantages over traditional uniaxial fibers, which are challenged with the long-term delivery of small hydrophilic molecules, in addition to providing transient and sustained-release simultaneously. We envision that future fiber architectures will localize active agents within specific sections of 
the fiber to tailor the release of individual agents independent of other encapsulants. Moreover, we anticipate that future platforms will combine architectures to maximize or complement the advantages of individual platforms. As previous clinical trials have shown, effective protection will be dependent upon fulfilling user preferences, offering convenience, and providing necessary release profiles from one vehicle, which fibers have the potential to realize. 


\section{CHAPTER 2}

IN VITRO STUDY ON SYNERGISTIC INTERACTIONS BETWEEN FREE AND ENCAPSULATED Q-GRIFFITHSIN AND ANTIRETROVIRALS AGAINST HIV-1 INFECTION

\section{Introduction}

Human immunodeficiency virus (HIV) remains a major health challenge, impacting over 1.7 million people each year ${ }^{1,2}$ and $\sim 38$ million worldwide. Women are disproportionately affected by HIV infections, and more than half of the globally infected patients are women living in sub-Saharan Africa ${ }^{3}$. In spite of eradication efforts, there is currently no cure or effective vaccine for HIV $-1^{1}$, hence a major emphasis has been placed on developing strategies to prevent and treat HIV-1 infection. Given the diversity and magnitude of these unmet reproductive health challenges, one focus has been to evaluate combinations of novel anti-HIV drugs delivered with multipurpose prevention technologies, to simultaneously target multiple sexually transmitted infections (STIs) ${ }^{4}$, such as herpes simplex virus 2 (HSV-2) and HIV-1.

One of the primary foci in preventing and treating HIV-1 infections, has been the development of antiretroviral drugs (ARVs) that inhibit HIV at different stages 
of the viral infection cycle $213-217$. Two ARVs currently used in clinical studies, Tenofovir (TFV) and Dapivirine (DAP), inhibit HIV-1 by blocking reverse transcriptase ${ }^{215}$, while Raltegravir (RAL), another approved drug for HIV treatment, inhibits proviral DNA-strand transfer ${ }^{218}$. Moreover, combinations of ARVs have demonstrated enhanced inhibition via different mechanisms of action ${ }^{219}$. While ARVs are effective in preventing HIV-1 infection, they have been shown to elicit adverse effects including bone marrow suppression and hematologic effects ${ }^{220}$, toxicity with long-term use, and an increased risk of antiviral resistance ${ }^{221}$. These adverse effects are often further exacerbated by the poor oral bioavailability and frequent dosing required for many of these drugs, which have contributed to modest outcomes in clinical trials 222 .

To help overcome these challenges, new biological agents have been investigated to provide protection via different mechanisms of action ${ }^{223}$. Antiviral lectins such as actinohivin, scytovirin, and microvirin have demonstrated efficacy against HIV-1 by binding to oligosaccharides linked to viral envelope glycoproteins; however, delivery of these lectins has been challenging due to low bioavailability and induction of an immune response ${ }^{224}$. Furthermore, toxicities arising from acute immunological response, due to the administration of foreign proteins, are one of the most significant concerns in adopting protein-based active agents ${ }^{225}$.

In comparison to these biologics, an antiviral lectin, Griffithsin, originally isolated from red alga Griffithsia sp. ${ }^{226}$, has demonstrated potent anti-HIV activity in the picomolar range against both laboratory and primary HIV isolates, and is currently being evaluated in clinical trials. Griffithsin inhibits HIV-1 by binding to an 
envelope glycoprotein (gp120) and inactivating HIV-1 almost immediately upon contact. Studies have shown that GRFT is stable and maintains activity in buffered solutions ( $\mathrm{pH} 4$ to 8 ) and cervical vaginal lavage fluid at $25^{\circ} \mathrm{C}$ and $37^{\circ} \mathrm{C}$ for up to 1 week $^{227}$. Additionally, GRFT exhibits excellent safety profiles $226,228-231$ with negligible induction of pro-inflammatory cytokines $^{231}$ and has demonstrated synergy with other antiretrovirals ${ }^{222}$, suggesting the benefits of future coadministration strategies. Recently, an oxidation-resistant variant of GRFT, QGRFT, was shown to retain the biophysical, antiviral, and safety properties of wildtype GRFT, making it an even more suitable HIV microbicide candidate 232,233 .

Prophylactic and therapeutic strategies that use a combination of anti-HIV drugs to target infection, have been shown to enhance the intracellular efficacy of each drug ${ }^{234,235}$ and decrease the transmission risk of antiviral-resistant viruses ${ }^{222}$. One study demonstrated that a combination of multiple nucleoside reverse transcriptase inhibitors (NRTIs) and non-nucleoside reverse-transcriptase inhibitors (NNRTIs), such as TFV and Efavirenz (EFV), provided synergistic inhibition of HIV-1, as well as an increase in intracellular drug efficacy ${ }^{235}$. Additionally, GRFT has shown synergistic effect in combination with maraviroc, TFV, and EFV, requiring lower GRFT doses when used in combination. The strongest synergy was observed between GRFT and TFV $(\mathrm{Cl}=0.34)$ suggesting that a combination of GRFT and other active agents, may provide a promising strategy to inhibit virus transmission and replication via different mechanisms of $\operatorname{action}^{222}$. 
Regardless of activity, active agents require formulation into an acceptable dosage form that protects agent stability and potentially provides extended release. Current $A R V s$ have been incorporated into short-acting or on-demand dosage forms such as tablets and gels, and long-acting sustained-release vehicles such as intravaginal rings (IVRs). However, oral ARVs incorporated in preexposure prophylaxis (PrEP) regimens have only been moderately successful in preventing, and to date do not cure HIV-1 infections. A variety of clinical studies ${ }^{236-}$ 240 have shown that the efficacy of oral PrEP ranges from zero to seventy-five percent, depending in large part on user adherence ${ }^{241}$.

As an alternative to daily oral PrEP administration, topical intravaginal PrEP technologies (e.g., gels, IVRs) have shown success in delivering active agents to the female reproductive tract (FRT). While clinical trials have demonstrated promising levels of protection for gels, efficacy has been shown to be significantly impacted or abbreviated by a lack of user adherence, due to the required frequent administration regimens and lack of retention attributed to product leakage ${ }^{242}$. In comparison, IVRs can provide long-term protection against STIs and pregnancy (> 1 month) by offering longer delivery durations and high durability in the FRT. However, clinical trials have reported some challenges regarding using IVRs such as vaginal discomfort, ease of product placement, low pliability, and hygiene issues ${ }^{243}$, further emphasizing the need to design alternative delivery platforms with potentially increased convenience and ease of use.

Thus far, short-term safety studies with GRFT have demonstrated outstanding preclinical safety in vitro and in vivo $231,244,245$; however currently, there 
are no available delivery vehicles that prolong GRFT (or Q-GRFT) delivery to achieve efficacy beyond $72 \mathrm{hr}$ post-administration. To begin to address these challenges, we and others have shown that polymeric nanoparticles (NPs) and electrospun fibers provide sustained-delivery, with the potential to safely incorporate and enhance the transport of biologics $40,128,130,246-265$. Polymeric NPs have been investigated to encapsulate $A R V s$ and biologics for HIV-1 treatment ${ }^{266-}$ 269 and have been shown to improve delivery to vaginal tissue by overcoming challenges such as agent instability; low cellular internalization and tissue distribution; and frequent dosing; while eliciting minimal immune response in the $\mathrm{FRT}^{154,268-271}$. Polymeric fibers that encapsulate active agents have also been recently applied to intravaginal drug delivery, demonstrating sustained-delivery and the potential to safely incorporate biologics ${ }^{128,272}$. Despite this, to date, there are no food and drug administration (FDA)-approved NP or fiber delivery platforms available to provide topical STI prevention ${ }^{128,252,273}$. Moreover, a platform that incorporates the attributes of both platforms, such as NP-fibers, may improve delivery by providing a reservoir to retain NPs and decrease NP clearance during shedding.

Despite being well-established technologies used successfully as durable stents, scaffolds, and delivery reservoirs for long-term implantation and delivery in vivo $260,274,275$, NP-fiber composites have only recently been explored for sustaineddelivery to the $\mathrm{FRT}^{273}$. Several groups have evaluated multilayered fibers for the delivery of active agents ${ }^{276,277}$, and have shown that nano- (often hydrophilic) and micro-scale (often hydrophobic or higher MW polymers) can impart greater 
mechanical stability and flexibility compared to a single layer of fibers ${ }^{278}$. Other work ${ }^{175,279,280}$ has investigated multilayered fibers and demonstrated timeregulated release of active agents. For intravaginal delivery specifically, an approach utilizing stacked fibers was evaluated for short-term (60 hr) intravaginal delivery, in part to demonstrate how individual layers pressed together postspinning may modulate release ${ }^{263}$. Recently, we developed a multilayered NP-fiber composite, that incorporates poly(lactic-co-glycolic) acid (PLGA) NPs, that sustains the delivery of GRFT in vitro, relative to sustained-delivery from NPs over $\sim 7$ days. In addition to the potential for sustained-delivery in the order of months, this NP-fiber architecture demonstrated efficacy against HIV-1 infections in vitro and protection against a lethal dose of HSV-2 in a murine model. Based on these studies, NP-fiber composites can be used to tailor the release of active agents and provide long-term efficacy.

In this work, we sought to examine the delivery of Q-GRFT and three different ARVs (e.g., TFV, RAL, and DAP) to identify unique protein-drug synergies between Q-GRFT and these active agents delivered in free or encapsulated forms. We selected these ARVs due to their different solubilities and mechanisms of action against HIV-1. TFV is a hydrophilic drug that has been evaluated in vaginal gels; DAP is a hydrophobic agent that has been incorporated in IVRs and evaluated in Phase III clinical trials and has been evaluated against both HIV-1 and HSV-2 infections, and RAL is a small hydrophilic molecule that is used late in pregnancy to reduce the risk of transferring HIV from mother to child ${ }^{281}$. We demonstrated that the co-administration of free or encapsulated active agents with 
different mechanisms of action may increase the prophylactic effect and decrease the dose needed to attain efficacy against HIV-1 infection. Moreover, given the potential of NP-based platforms to modulate active agent release, we sought to preliminarily assess the release of different active agent agents from NPs, and selected the most synergistic NP formulations for incorporation in multilayered NPfiber composites.

\section{Materials and Methods}

Poly(lactic-co-glycolic) acid used for synthesizing NPs (50:50, 0.55-0.75 $\mathrm{dL} / \mathrm{g}, 31-57 \mathrm{k} \mathrm{MW}$ ) was purchased from Lactel Absorbable Polymers (Cupertino, CA). Polycaprolactone ( $\mathrm{PCL}, \mathrm{MW}=80,000 \mathrm{Da}$ ), polyethylene oxide (PEO, $\mathrm{MW}=600,000 \mathrm{Da}), \mathrm{TFV}$ and DAP were purchased from Sigma Aldrich (St Louis, MO), while RAL was provided from Selleckchem (Houston, TX). Q-GRFT was kindly provided by Dr. Fuqua (University of Louisville, KY). Dichloromethane (DCM) and keratinocyte serum-free medium (KSFM) were purchased from Thermo Fisher (Waltham, MA). Other chemicals, including dimethyl sulfoxide (DMSO), acetonitrile HPLC grade (ACN), trifluoroacetic acid (TFA), and thiazolyl blue tetrazolium bromide (MTT), were obtained from Sigma Aldrich.

\section{Nanoparticle Fabrication}

Blank NPs and NPs loaded with Q-GRFT, TFV, RAL or DAP were fabricated using either single or double emulsion technique. For each formulation, $100 \mathrm{mg}$ PLGA was dissolved in $2 \mathrm{~mL}$ of DCM and incubated overnight. For Q-GRFT, TFV, and RAL NPs, $10 \mathrm{mg}$ of the active agent was dissolved in $200 \mu \mathrm{L}$ Tris-EDTA (TE) 
buffer. For DAP NPs, $10 \mathrm{mg}$ of DAP was dissolved in $200 \mu \mathrm{L}$ DCM. The active agent solution was added dropwise to the polymer solution while the solution was vortexed. Then, the active agent-polymer solution was sonicated and added to $5 \%$ PVA in Milli-Q water (2 mL), vortexed and sonicated again. The emulsified solution was then added to a larger volume of aqueous solution ( $50 \mathrm{~mL}$ of $0.3 \%$ PVA) and incubated for $3 \mathrm{hr}$ while stirring to evaporate DCM. Then, NPs were centrifuged $\left(25,364 \mathrm{xg}\right.$ for $10 \mathrm{~min}$ at $\left.4^{\circ} \mathrm{C}\right)$, washed twice using $30 \mathrm{~mL}$ Milli-Q water, suspended in $5 \mathrm{~mL}$ Milli-Q water and stored at $-80^{\circ} \mathrm{C}$ for $2 \mathrm{hr}$. Finally, NPs were lyophilized to obtain solid particles and were subsequently stored at $-20^{\circ} \mathrm{C}$.

\section{NP-Fiber Composite Fabrication}

To prepare the electrospinning solution, PEO and PCL were dissolved in DI water $(5 \% \mathrm{w} / \mathrm{w})$ and TFE $(11 \% \mathrm{w} / \mathrm{w})$, respectively, and incubated at $37^{\circ} \mathrm{C}$ overnight. Prior to electrospinning, $10 \% \mathrm{w} / \mathrm{w}$ of PLGA NPs were added to the PEO solution and the solution was electrospun with a flow rate of $0.3 \mathrm{~mL} / \mathrm{hr}$ and voltage of $25 \mathrm{kV}$. Pre-cut NP-PEO fibers (3-5 mg) were placed on the freshly electrospun PCL layer followed by electrospinning another layer of PCL fiber on top to make a sandwich-shaped structure. The electrospinning conditions for PCL layers were set to $15 \mathrm{~cm}$ needle to collector distance, flow rate of $2.2 \mathrm{~mL} / \mathrm{hr}$, and voltage of 20 $\mathrm{kV}$. The NP-fiber composite was desiccated overnight and stored at $4^{\circ} \mathrm{C}$.

\section{NP Characterization: Size, Loading, and Release}

Size. The size and morphology of unhydrated NPs were determined using scanning electron microscopy (SEM). First, samples were sputter-coated with a 
palladium/gold alloy layer with a thickness of 15 to $18 \mathrm{~nm}$, and imaged using a Supra 35 SEM (Zeiss, Oberkochen, Germany). The average size of unhydrated NPs was evaluated with Image J software ( $\mathrm{NIH}$, Bethesda, Maryland) by measuring the diameter of 50 NPs per image and 3 images for each formulation.

Loading. To assess the loading of NPs, 1-2 mg of NPs were dissolved in $100 \mu \mathrm{L}$ DMSO and diluted with TE buffer to the final volume of $1 \mathrm{~mL}$. Serial 1:2 sample dilutions were then made in TE buffer. To quantify the amount of TFV, RAL, and DAP, spectrophotometric absorbance was measured at 260, 300 and $290 \mathrm{~nm}$, respectively, on a Synergy HT reader (BioTek, Winooski, VT, USA). To quantify the amount of Q-GRFT, an enzyme-linked immunosorbent assay (ELISA) was used. Briefly, 96-well Nunc Maxisorp plates were incubated with $100 \mu \mathrm{L}$ of $250 \mathrm{ng} / \mathrm{mL}$ gp120 in 1x phosphate-buffered saline (PBS) as a coating buffer and stored at $4^{\circ} \mathrm{C}$ overnight. Then, plates were blocked with $300 \mu \mathrm{L}$ of $3 \% \mathrm{w} / \mathrm{v}$ bovine serum albumin (BSA) in PBS containing 0.05\% Tween-20 (1x PBST) and incubated at room temperature for $2 \mathrm{hr}$. Plates were then washed using a Gardner Denver Multiwash III plate washer (Milwaukee, WI) followed by adding $100 \mu \mathrm{L}$ of the samples. Afterwards, $100 \mu \mathrm{L}$ of rabbit anti-GRFT $(1: 10,000)$ and goat antirabbit IgG-HRP $(1: 20,000)$ were subsequently added after $1 \mathrm{hr}$ incubation at $37^{\circ} \mathrm{C}$ to detect gp120-bound Q-GRFT. Finally, $100 \mu \mathrm{L}$ of SureBlue TMB microwell peroxidase substrate (Sera Care, Milford, MA, USA) was added to each well and the reaction was quenched by adding $100 \mu \mathrm{L}$ of $1 \mathrm{~N} \mathrm{H}_{2} \mathrm{SO}_{4}$ (Thermo Fisher) followed by reading the absorbance at $450 \mathrm{~nm}$. 
In Vitro Release. The release of Q-GRFT, TFV, RAL, and DAP loaded PLGA NPs was determined over 30 days in simulated vaginal fluid (SVF, pH 4.5). Three to five $\mathrm{mg}$ of NPs were dispersed in $1 \mathrm{~mL}$ of SVF in $1.5 \mathrm{~mL}$ centrifuge tubes and incubated at $37^{\circ} \mathrm{C}$ with continuous shaking (150 rpm). At each time point, samples were centrifuged at $18,500 \times \mathrm{g}$ at $4^{\circ} \mathrm{C}$ for $10 \mathrm{~min}$, the eluates were collected, and NPs were resuspended in $1 \mathrm{~mL}$ of fresh SVF. Similarly, the release of the active agent from NP-fiber constructs was evaluated by placing a $5 \mathrm{mg}$ fiber composite piece in a $5 \mathrm{~mL}$ microcentrifuge tube filled with $1 \mathrm{~mL}$ SVF. The samples were incubated on a shaker at $150 \mathrm{rpm}$ at $37^{\circ} \mathrm{C}$, under sink conditions. At each time point, the solution was replaced with fresh SVF and release from NP-fiber composites was assessed for up to 90 days. The release of Q-GRFT, from NPs and NP-fiber composites, was quantified via ELISA as mentioned before. For quantifying DAP released from NP-fiber composites, reverse phase high performance liquid chromatography with UV detection (HPLC-UV) was used (Agilent, CA, USA). First, the composite eluates in SVF were diluted with ACN to obtain a 1:1 SVF:ACN v:v ratio. Then, $50 \mu \mathrm{L}$ of sample was injected into a Jupiter C18 300 A-C18 column $(250 \times 4.6 \mathrm{~mm}$; Agilent Technologies, CA, USA $)$. The mixture of $65 \%$ of $0.01 \mathrm{v} / \mathrm{v} \%$ TFA and $35 \%$ ACN was used as mobile phase with the flow rate of $1 \mathrm{~mL} / \mathrm{min}$ and the running time for DAP was $22 \mathrm{~min}$. Finally, DAP was detected via absorbance at $290 \mathrm{~nm}$ and the concentration of DAP in unknown samples was determined by plotting the area under the curve versus concentration. The standard curve over the range of 0.08 to $10 \mu \mathrm{g} / \mathrm{mL}$ was determined to be linear. 


\section{Cell Lines, Virus and Cell Culture}

TZM-bl cells were obtained from the NIH AIDS Research and Reference Reagent Program (ARRRP) and were used for HIV infection. These cells are engineered HeLa cells that express CD4, CCR5, and CXCR4 receptors and have Tat-responsive reporter genes for firefly luciferase (Luc) that allows accurate measuring of HIV-1 infection 234 . TZM-bl cells were maintained in Dulbecco's Modified Eagle medium (DMEM, from VWR) supplemented with $10 \%$ fetal bovine serum (FBS), $25 \mathrm{mM}$ HEPES buffer, and $50 \mu \mathrm{g} / \mathrm{mL}$ gentamicin (Thermo Fisher). Vaginal epithelial (VK2/E6E7), End1/E6E7 (End1), and ectocervical, Ect1/E6E7 (Ect1) cell lines were provided courtesy of Dr. Kenneth Palmer from the University of Louisville, and were originally from ATCC (Rockville, MD). VK2, End 1, and Ect 1 cells were cultured in Keratinocyte Serum-Free (KSFM) media containing recombinant human epidermal growth factor $(0.1 \mathrm{ng} / \mathrm{mL})$, bovine pituitary extract (50 $\mu \mathrm{g} / \mathrm{mL})$, calcium chloride $(0.4 \mathrm{mM})$ (Thermo Fisher), and $1 \%$ penicillin and streptomycin. HEK-293T/17 cells (ATCC, Rockville, MD) were used for HIV production and were cultured in minimum essential medium (MEM) supplemented with $10 \%$ FBS, and $1 \%$ penicillin $(100 \mu \mathrm{g} / \mathrm{mL})$ and streptomycin $(100 \mu \mathrm{g} / \mathrm{mL})(V W R$ Radnor, PA). The HIV-1 pseudovirus was made in house by transfecting HEK293T/17 cells with envelope (env)-expressing plasmid (CCR5-tropic clade A strain, Q769.h5), env-deficient HIV-1 backbone vector (HIV-1pNL4-3.Luc.R-E-, NIH AIDS Reagent Program,11884 and 3418) and Mirus reagent (Mirus, Madison, WI ). After incubating cells with transfection complex for $72 \mathrm{hr}$ at $37^{\circ} \mathrm{C}$, the cells

were centrifuged ( $200 \times \mathrm{g}, 5 \mathrm{~min})$ and the supernatant containing viral particles 
was collected, filtered using a $0.45 \mu \mathrm{m}$ membrane filter (VWR), and stored at $80^{\circ} \mathrm{C}$.

\section{In Vitro HIV Inhibition}

To evaluate the anti-HIV-1 activity of anti(retro)viral NPs, an in vitro HIV inhibition assay was conducted by infecting TZM-bl cells with HIV-1 pseudovirus (CCR5-using clade A strain Q769.h5) and quantifying the luciferase activity of cell lysates. Briefly, the desired amount of free or encapsulated active agent was dissolved in $1 \mathrm{~mL}$ of DMEM, followed by 1:2 serial dilutions to a final volume of $50 \mu \mathrm{L}$. Then, $100 \mu \mathrm{L}$ of cell solution $\left(10^{6}\right.$ cells per plate) was added to each well. After incubating the cells and treatment for $1 \mathrm{hr}$ at $37^{\circ} \mathrm{C}, 50 \mu \mathrm{L}$ of virus solution was added to each well. Untreated cells only and virus-infected cells were used as negative and positive controls of infection, respectively. After $48 \mathrm{hr}$ incubation at $37^{\circ} \mathrm{C}, 100 \mu \mathrm{L}$ culture medium was replaced with $100 \mu \mathrm{L}$ Bright-Glo reagent solution (Promega Corporation, Madison, WI) and luminescence was measured after 3 min. HIV-1 infection was determined based on the luminescence deviations from the virus-infected cell control.

\section{In Vitro Cytotoxicity}

An MTT assay was used to determine the cytotoxicity of blank and anti(retro)viral NPs (0.1 and $1 \mathrm{mg} / \mathrm{mL}$ ) administered to VK2, End1, and Ect1/E6E7 cell lines. First, cells were plated at a density of 150,000 cells/well in 24 -well plates and incubated for $24 \mathrm{hr}$ at $37^{\circ} \mathrm{C}$, followed by adding treatments in triplicate. Media only (no treatment) and 10\% DMSO were used as viable and non-viable cell 
controls. After 24,48 , and $72 \mathrm{hr}$ incubation at $37^{\circ} \mathrm{C}, 55 \mu \mathrm{L}$ of MTT reagent was added to each well and incubated at $37^{\circ} \mathrm{C}$ for $4 \mathrm{hr}$, followed by adding $100 \mu \mathrm{L}$ of lysis buffer containing $10 \%$ sodium dodecyl sulfate (Fisher Scientific) and $0.01 \mathrm{M}$ hydrochloric acid. After overnight incubation, the absorbance was read at $570 \mathrm{~nm}$ and normalized to cell-only absorbance to attain the relative percent of cell viability.

\section{Combination Effect}

The activity of the active agents in combination was determined using the combination index $(\mathrm{Cl})$. First, the half-maximal inhibitory concentration $\left(\mathrm{IC}_{50}\right)$ value of each drug in free and encapsulated form was measured with the HIV inhibition assay. Then, each drug was mixed with either Q-GRFT or Q-GRFT NPs in an equipotent $\mathrm{IC}_{50}$ ratio $\left(1: 1\right.$ ratio of $\left.\mathrm{IC}_{50}\right)$. For NPs, the amount of each active agent was determined based on the actual loading. After measuring the $\mathrm{IC}_{50}$ values of each active agent in combination, the Chou-Talalay method was used to measure $\mathrm{Cl}^{282-284}$.

(Eqn. 1) $C I=\frac{D_{C 1}}{D_{S 1}}+\frac{D_{C 2}}{D_{S 2}}+\frac{D_{C 1} D_{C 2}}{D_{S 1} D_{S 2}}$

When Q-GRFT and the drug are in free form, Ds1 and Ds2 represent the $\mathrm{IC}_{50}$ of free Q-GRFT and the free drug applied separately, while $\mathrm{Dc}_{\mathrm{c}}$ and $\mathrm{Dc}_{\mathrm{c} 2}$ are the $\mathrm{IC}_{50}$ values of free Q-GRFT and free drug applied in combination, respectively. When Q-GRFT NPs are co-administered with free drug, Ds 1 and Ds2 represent the $I_{50}$ of Q-GRFT NPs and the free drug when applied separately, while $D_{c 1}$ and $D_{c 2}$ are the $\mathrm{IC}_{50}$ values of Q-GRFT NPs and free drug when they are co-administered. Finally, in the case of Q-GRFT NPs and encapsulated drug co-administration, Ds1 
and Ds2 represent the $\mathrm{IC}_{50}$ of Q-GRFT NPs and drug NPs, while $\mathrm{Dc}_{\mathrm{c}}$ and $\mathrm{D}_{\mathrm{c} 2}$ are the IC 50 values of Q-GRFT NPs and drug NPs in combination, respectively. Values of $\mathrm{Cl}>1, \mathrm{Cl}=1$, and $\mathrm{Cl}<1$ determine additive, antagonistic, and synergistic interactions, respectively.

\section{Statistical Analysis}

All experiments were done in triplicate and GraphPad Prism (GraphPad Software, La Jolla, CA) was used for data analysis. Unless otherwise noted, three independent replicates were assessed for each sample and statistical significance was evaluated using a one-way ANOVA test (Bonferroni), and $p$-values $\leq 0.05$ were considered statistically significant.

\section{Results}

\section{NP Characterization}

PLGA NPs formulated with $100 \mu \mathrm{g}$ of ARVs (TFV, RAL, or DAP) or Q-GRFT per mg of NP were successfully synthesized with high yield (> 70\%) and spherical morphology. SEM images of ARV and Q-GRFT NPs are shown in Figure 2.1, demonstrating NP sizes ranging from 98.9 to $107.2 \mathrm{~nm}$. Furthermore, nanoparticle loading efficiency varied with the active agent (Table 2.1); Q-GRFT, TFV, RAL, and DAP NPs were loaded with $48.9 \pm 12.7,15.4 \pm 3.3,103.5 \pm 11.9$, and $60.2 \pm$ 2.7 $\mu \mathrm{g}$ active agent/mg NP, respectively, directly corresponding to the encapsulation efficiency. 

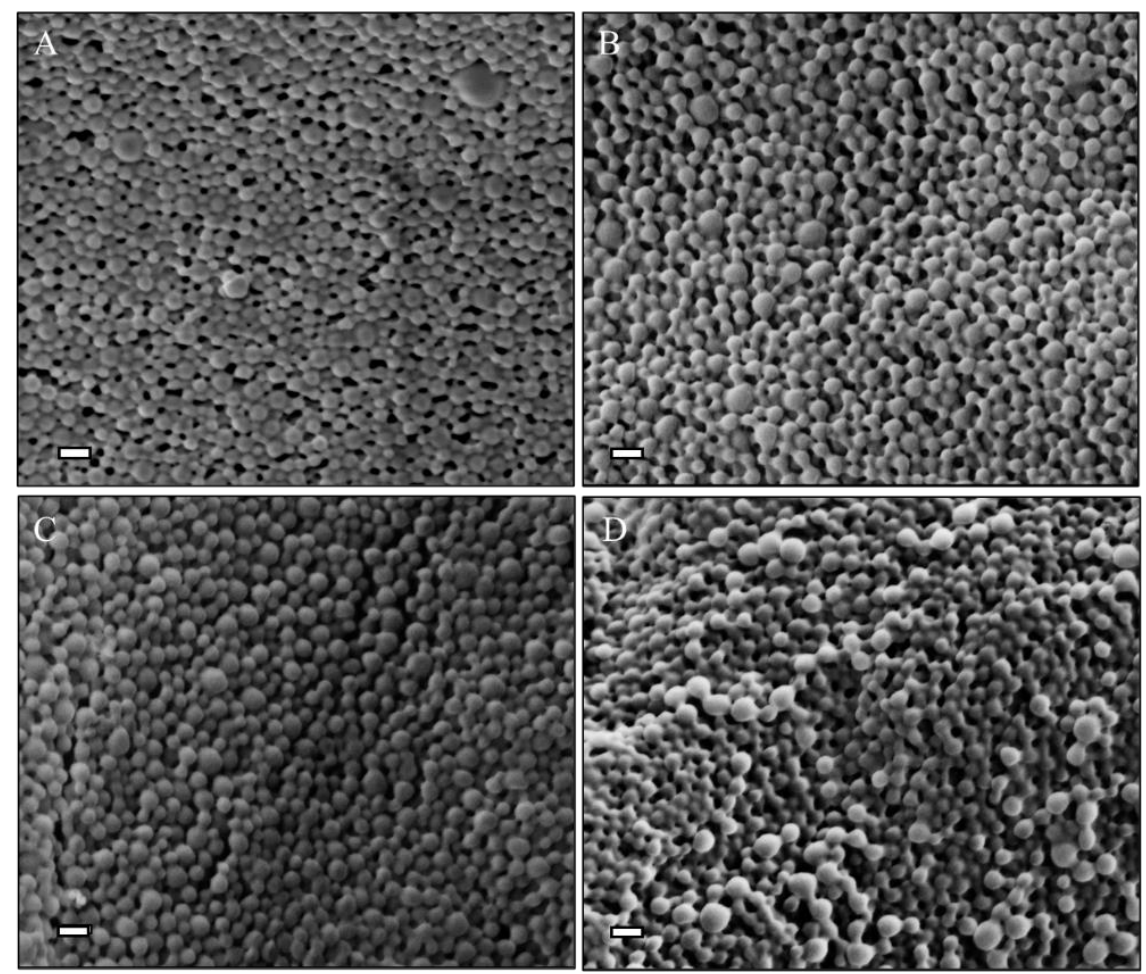

Figure 2.1. Scanning electron microscopy images of PLGA nanoparticles loaded with 10\% w/w (A) Q-GRFT, (B) TFV, (C) RAL, and (D) DAP. Scale bars represent $200 \mathrm{~nm}$.

Table 2.1. Size, loading, and encapsulation efficacy of PLGA nanoparticles. Nanoparticles had theoretical loading values of $100 \mu \mathrm{g}$ Q-GRFT, TFV, RAL, or DAP per mg NP.

\begin{tabular}{|c|c|c|c|}
\hline Active Agent & Size $(\mathbf{d} . \mathbf{n m} \pm \mathbf{S T D})$ & Loading $(\boldsymbol{\mu g} / \mathbf{m g})$ & Encapsulation Efficacy $(\boldsymbol{\%})$ \\
\hline Q-GRFT & $98.5 \pm 37.2$ & $48.9 \pm 12.7$ & $48.9 \pm 12.7$ \\
\hline TFV & $89.9 \pm 25.3$ & $15.4 \pm 3.3$ & $15.4 \pm 3.3$ \\
\hline RAL & $106.3 \pm 29.7$ & $103.5 \pm 11.9$ & $103.5 \pm 11.9$ \\
\hline DAP & $107.2 \pm 27.1$ & $60.2 \pm 2.7$ & $60.2 \pm 2.7$ \\
\hline
\end{tabular}




\section{In Vitro Release from NPs}

The cumulative release of each active agent as a function of mass and percent total loading, from PLGA NPs over 4 wk in SVF is shown in Figure 2.2. For all formulations, a burst release was observed within the first eight hours, followed by a more gradual release over 2 wk. Specifically, 2.51, 1.72, 8.96, and $8.99 \mu \mathrm{g}$ Q-GRFT, TFV, RAL, and DAP per mg of NPs (representing $5 \%, 11 \%, 9 \%$, and $15 \%$ of actual loading) were released after $8 \mathrm{hr}$. Over $2 \mathrm{wk}$, the total cumulative release of Q-GRFT, TFV, RAL, and DAP from their respective NP formulations was $4.64,2.64,11.84$, and $13.41 \mu \mathrm{g} / \mathrm{mg}$, resulting in less than $20 \%$ release of each active agent.
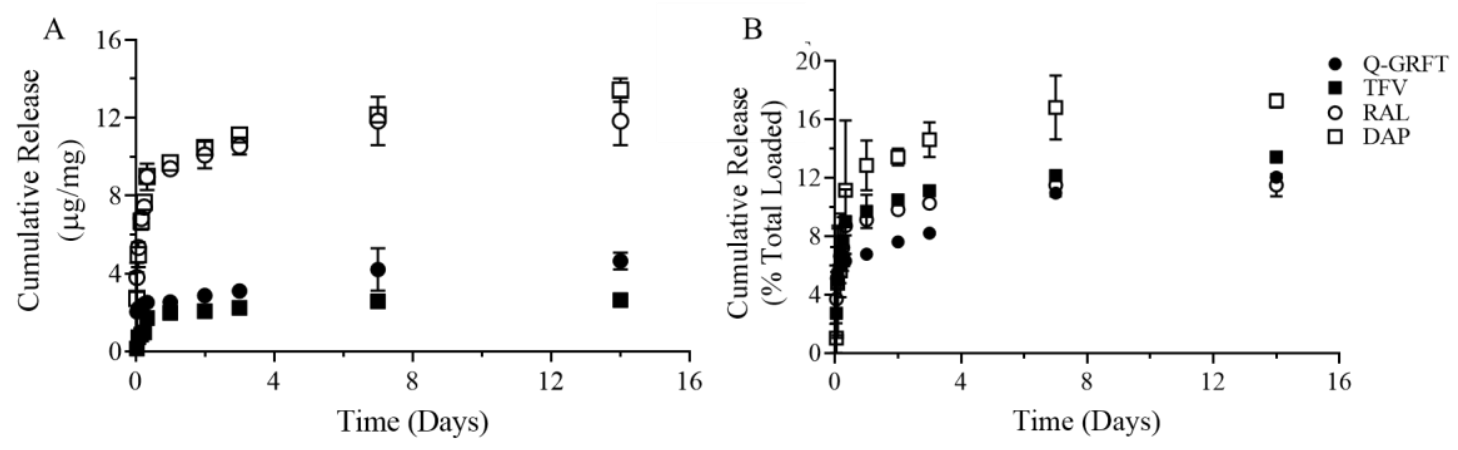

Figure 2.2. The cumulative release of $Q-G R F T, T F V, R A L$, and DAP from PLGA nanoparticles as a function of $(A)$ total active agent release or $(B)$ the percent of total loading, after exposure to SVF for up to $14 \mathrm{~d}$. Release values are shown as the mean \pm standard deviation of three independent NP batches. Please note panel $\mathrm{B} y$-axis is scaled to $20 \%$ to more easily visualize differences in release. 


\section{In Vitro HIV-1 Inhibition Studies}

\section{Individual administration of Q-GRFT or ARVs in free or encapsulated}

form. The ability of individual active agents to protect against infection in free and encapsulated forms was evaluated by the administration of free active agent to TZM-bl cells for $1 \mathrm{hr}$, followed by virus administration. The IC 50 values of free QGRFT, TFV, RAL, and DAP were $57.2 \pm 13.8,382.3 \pm 39.6,3.3 \pm 0.2$, and 0.034 $\pm 0.008 \mathrm{ng} / \mathrm{mL}$, respectively, while the encapsulation of individual in agents in NPs generally decreased the $\mathrm{IC}_{50}$ values to $14.0 \pm 4.1,273.7 \pm 78.8,3.0 \pm 0.5$, and $0.043 \pm 0.0007 \mathrm{ng} / \mathrm{mL}$ (Figure 2.3). For all ARVs, the $\mathrm{IC}_{50}$ values of free and encapsulated agents based on release were similar $(p>0.05)$, while the $\mathrm{IC}_{50}$ values of Q-GRFT NPs were $~ 4$-fold lower than observed for free Q-GRFT ( $p \leq$ 0.05) (Table 2.2). 

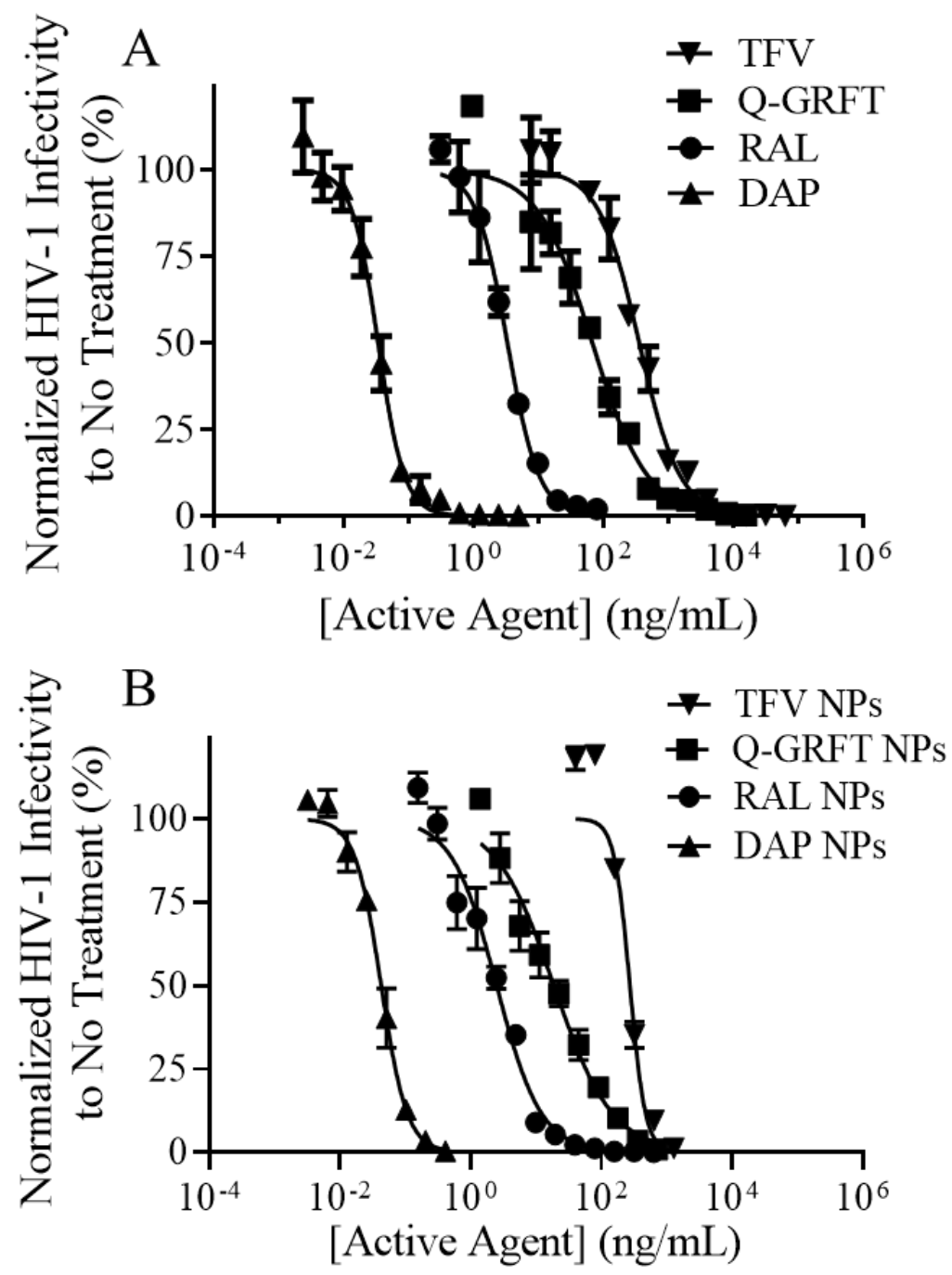

Figure 2.3. The $\mathrm{IC}_{50}$ curves of $(\mathrm{A})$ free and (B) NP-encapsulated active agents after administration to TZM-bl cells $1 \mathrm{hr}$ prior to HIV-1 pseudovirus infection. Infectivity values are normalized to uninfected cells and are shown as the mean \pm standard deviation of three NP batches. 
Table 2.2. I $\mathrm{I}_{50}$ values of free and encapsulated agents after administration to TZM-bl cells $1 \mathrm{hr}$ before HIV-1 pseudovirus infection. Data are shown in units of $\mathrm{ng} / \mathrm{mL}$ and $\mathrm{nM}$.

\begin{tabular}{|c|c|c|c|c|}
\hline Active Agent & $\begin{array}{c}\mathbf{I C}_{\mathbf{5 0}} \text { Free Active Agent } \\
(\mathbf{n g} / \mathbf{m L})\end{array}$ & $\begin{array}{c}\mathbf{I C}_{\mathbf{5 0}} \text { Free Active Agent } \\
(\mathbf{n M})\end{array}$ & $\begin{array}{c}\mathbf{I C}_{\mathbf{5 0}} \text { Encapsulated } \\
\text { Active Agent }(\mathbf{n g} / \mathbf{m L})\end{array}$ & $\begin{array}{c}\mathbf{I C}_{\mathbf{5 0}} \text { Encapsulated } \\
\text { Active Agent (nM) }\end{array}$ \\
\hline Q-GRFT & $57.2 \pm 13.8$ & $4.5 \pm 1.1$ & $14.0 \pm 4.1$ & $1.1 \pm 0.3$ \\
\hline TFV & $382.3 \pm 39.6$ & $1329.6 \pm 137.8$ & $273.7 \pm 78.8$ & $962.6 \pm 274.3$ \\
\hline RAL & $3.3 \pm 0.3$ & $7.4 \pm 0.7$ & $3.0 \pm 0.5$ & $6.7 \pm 1.1$ \\
\hline DAP & $0.034 \pm 0.008$ & $0.1 \pm 0.024$ & $0.043 \pm 0.0007$ & $0.1 \pm 0.002$ \\
\hline
\end{tabular}

Combinations of Q-GRFT with each ARV. Q-GRFT was subsequently coadministered with each $\mathrm{ARV}$ at an equipotent $\mathrm{IC}_{50}$ value ratio to compare the combined efficacy with each active agent. As shown in Figure 2.4, when free QGRFT was co-administered with free ARVs, all IC 50 curves were shifted to the left, demonstrating that a lower dose of each agent is able to achieve similar efficacy. When free Q-GRFT was co-administered with free TFV, RAL, or DAP, the IC $\mathrm{C}_{50}$ of Q-GRFT decreased by $6.3,4.4$, and 3.7 -fold respectively, relative to the administration of $Q-G R F T$ alone $(p \leq 0.05)$. Similarly, the $I_{50}$ values of free TFV, RAL, or DAP co-administered with free Q-GRFT decreased by 2.8, 5.6, and 4.6fold respectively, relative to the administration of free drug alone $(p \leq 0.05)$. 

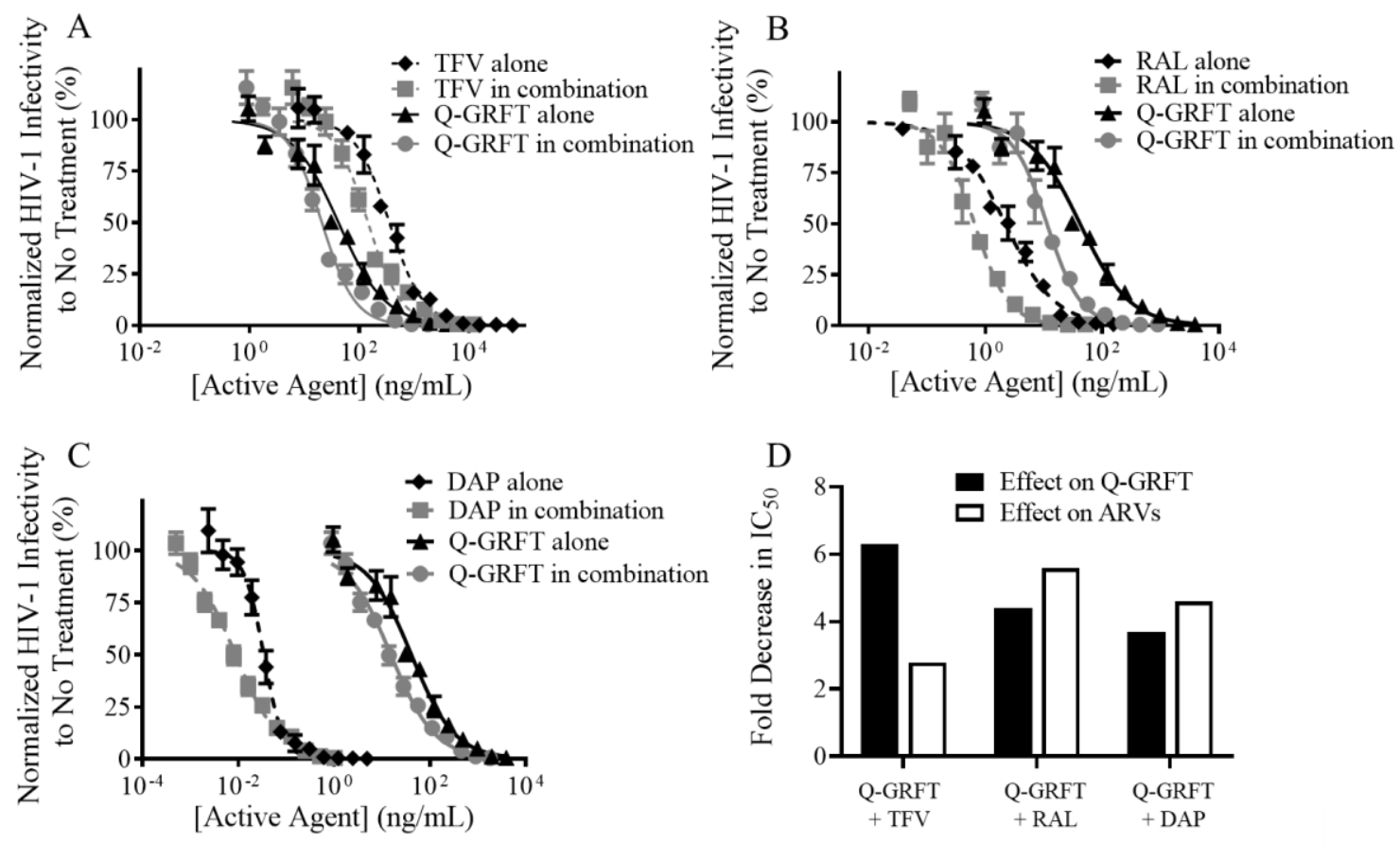

Figure 2.4. The $I_{50}$ curves for free $A R V s$ and free $Q-G R F T$ co-administration demonstrate synergistic interactions. (A) Free TFV + free Q-GRFT, (B) free RAL + free Q-GRFT and (C) free DAP + free Q-GRFT. (D) Fold decrease in the $\mathrm{IC}_{50}$ values of Q-GRFT and ARVs after co-administration to TZM-bl cells $1 \mathrm{hr}$ prior to HIV-1 pseudovirus infection. The normalized infectivity values are shown as the mean \pm standard deviation of three independent samples. Please note differences in log scale on the x-axis.

Combinations of Q-GRFT NPs with each ARV. The activity Q-GRFT NPs with each free ARV was assessed using the equipotency ratio of Q-GRFT NPs to each ARV, with the IC 50 of Q-GRFT NPs calculated based on the loading value. As shown in Figure 2.5, the IC 50 values of Q-GRFT NPs decreased by 6.6, 4.3, and 2.2-fold when co-administered with free TFV, RAL, or DAP, respectively, relative to the administration of Q-GRFT NPs alone. The $\mathrm{IC}_{50}$ values of free TFV 
and RAL co-administered with free Q-GRFT NPs decreased by 1.6 and 1.4-fold, relative to free TFV and RAL; however, minimal reduction ( 0.5 -fold) was observed in the $\mathrm{IC}_{50}$ value of DAP.

Figure 2.5. The $\mathrm{IC}_{50}$ curves for free drugs and Q-GRFT NP co-administration
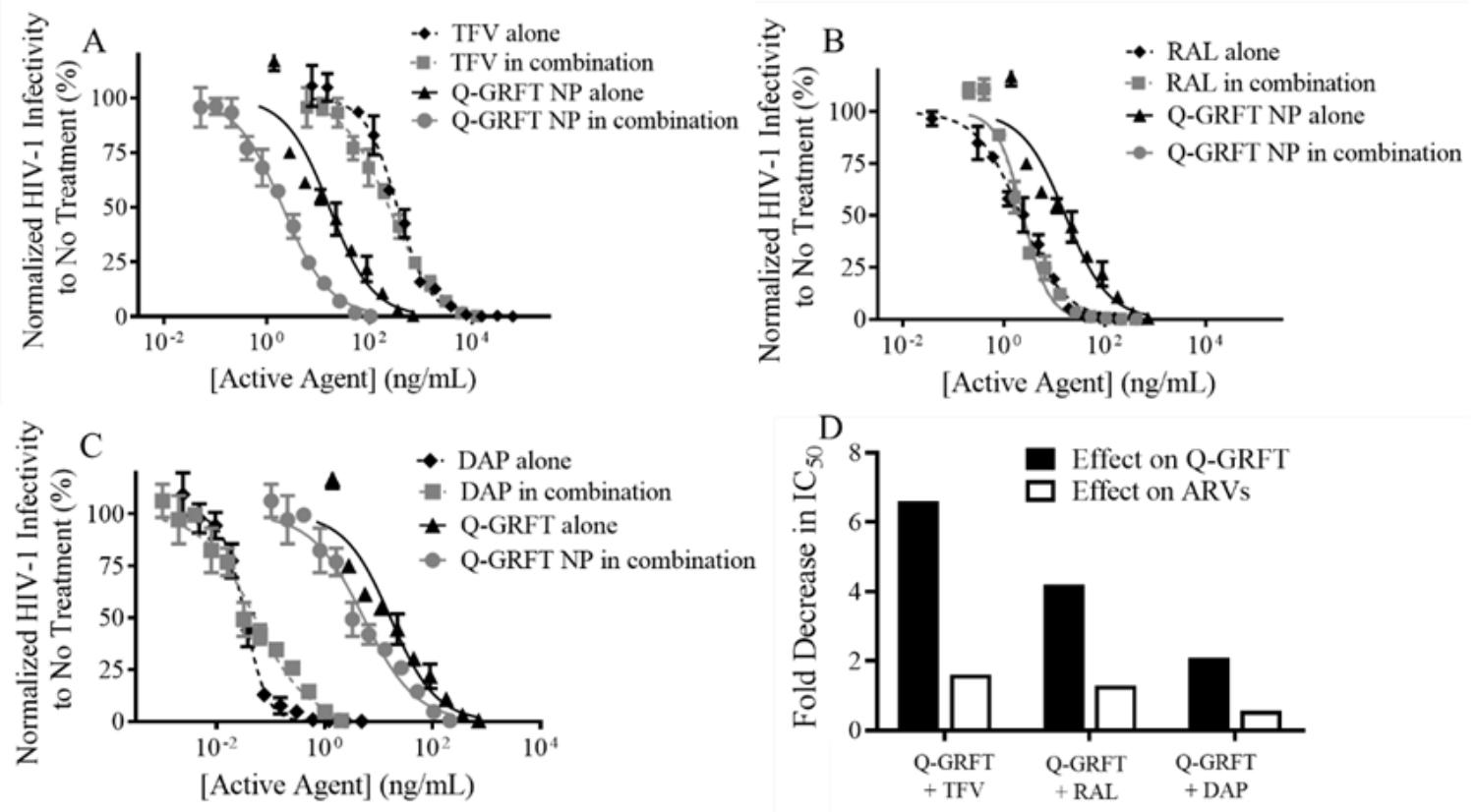

demonstrate synergistic interactions. (A) Free TFV + Q-GRFT NPs, (B) free RAL + Q-GRFT NPs and (C) free DAP + Q-GRFT NPs. (D) Fold decrease in the IC $\mathrm{C}_{50}$ values of Q-GRFT NPs and ARVs after co-administration to TZM-bl cells $1 \mathrm{hr}$ prior to HIV-1 pseudovirus infection. The normalized infectivity values are shown as the mean \pm standard deviation of three independent samples. Please note differences in log scale on the x-axis.

Combinations of Q-GRFT NPs with ARV NPs. Lastly, the efficacy of QGRFT NPs and individual ARV NP groups was determined using the equipotency ratio of Q-GRFT NPs to each ARV NP based on loading values. A significant shift was observed in the IC50 curves after Q-GRFT NP and ARV NP co-administration 
(Figure 2.6). Specifically, a 2.5, 2.7, and 6.0-fold reduction in Q-GRFT NP IC 50 values was observed when they were co-administered with TFV NPs, RAL NPs, or DAP NPs. In addition, co-administration of TFV NPs, RAL NPs, or DAP NPs with Q-GRFT NPs resulted in 3.3, 2.3, and 8.6-fold reduction in the $\mathrm{IC}_{50}$ of each encapsulated drug in combination, relative to each encapsulated drug alone. In general, although all NPs showed synergistic interactions when administered at an equipotency ratio, the most synergistic interactions were observed for DAP NPs and Q-GRFT NPs, resulting in 8.6 and 6.1-fold decreases in $\mathrm{IC}_{50}$ respectively, relative to individual DAP NPs and Q-GRFT NPs. The summary of $\mathrm{IC}_{50}$ values of different active agents administered individually, relative to the $I_{50}$ values after co-administration in free and encapsulated forms, is shown in Table 2.3. 

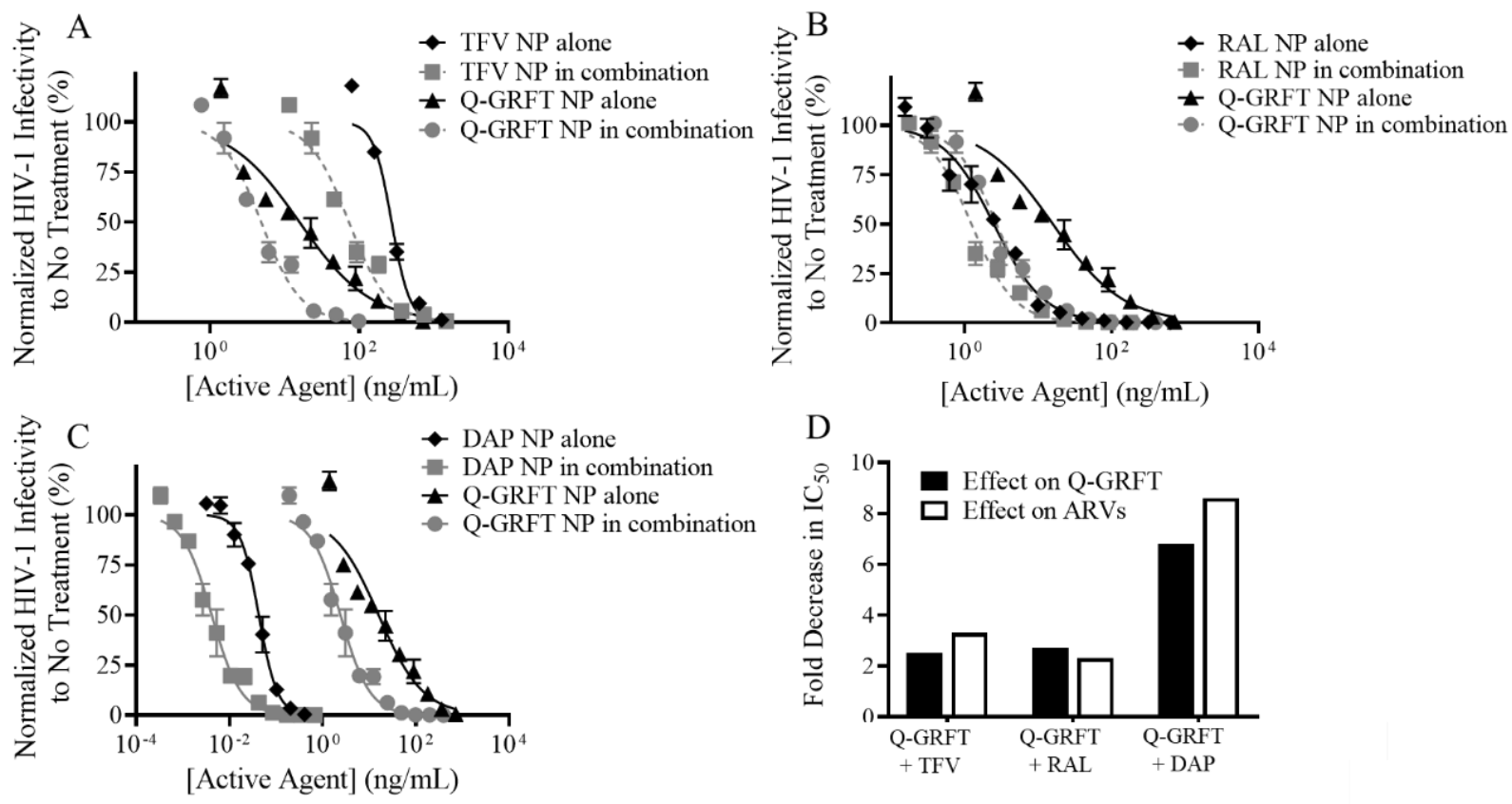

Figure 2.6. The $I_{50}$ curves for ARV NP and Q-GRFT NP co-administration. (A) TFV NPs + Q-GRFT NPs, (B) RAL NPs + Q-GRFT NPs and (C) DAP NPs + QGRFT NPs. (D) Fold decrease in the $\mathrm{IC}_{50}$ values of Q-GRFT NPs and ARV NPs after co-administration to TZM-bl cells $1 \mathrm{hr}$ prior to HIV-1 pseudovirus infection. The normalized infectivity values are shown as the mean \pm standard deviation of three independent samples. Please note differences in log scale on the $x$-axis. 
Table 2.3. Summary of the $\mathrm{IC}_{50}$ values of each individual active agent after coadministration in different forms. Active agents were administered to TZM-bl cells $1 \mathrm{hr}$ before HIV-1 pseudovirus infection.

\begin{tabular}{|c|c|c|c|c|c|c|}
\hline \multicolumn{7}{|c|}{ IC $_{\mathbf{5 0}}$ of Active Agents after Co-administration $(\mathbf{n g} / \mathbf{m L})$} \\
\hline \multirow{2}{*}{ Active Agents } & Free ARV + Free Q-GRFT & \multicolumn{1}{|c|}{ Free ARV + Q-GRFT NP } & \multicolumn{1}{|c|}{ ARV NP + Q-GRFT NP } \\
\cline { 2 - 7 } & ARV & Q-GRFT & ARV & Q-GRFT & ARV & Q-GRFT \\
\hline Q-GRFT+TFV & $135.4 \pm 7.0$ & $9.1 \pm 2.2$ & $244.4 \pm 21.3$ & $2.1 \pm 0.1$ & $83.3 \pm 10.3$ & $5.5 \pm 0.7$ \\
\hline Q-GRFT+RAL & $0.6 \pm 0.1$ & $12.9 \pm 2.0$ & $2.4 \pm 0.1$ & $3.3 \pm 1.2$ & $1.3 \pm 0.1$ & $5.2 \pm 0.2$ \\
\hline Q-GRFT+DAP & $0.00737 \pm 0.006$ & $15.4 \pm 0.1$ & $0.06 \pm 0.02$ & $6.5 \pm 2.0$ & $0.005 \pm 0.001$ & $2.3 \pm 0.1$ \\
\hline
\end{tabular}

\section{Evaluation of Synergistic Interactions based on Cl Calculations}

The $\mathrm{Cl}$ value was calculated using the Chou-Talalay method based on the ratio of $\mathrm{IC}_{50}$ values for each active agent when administered separately and in combination (Figure 2.7). 


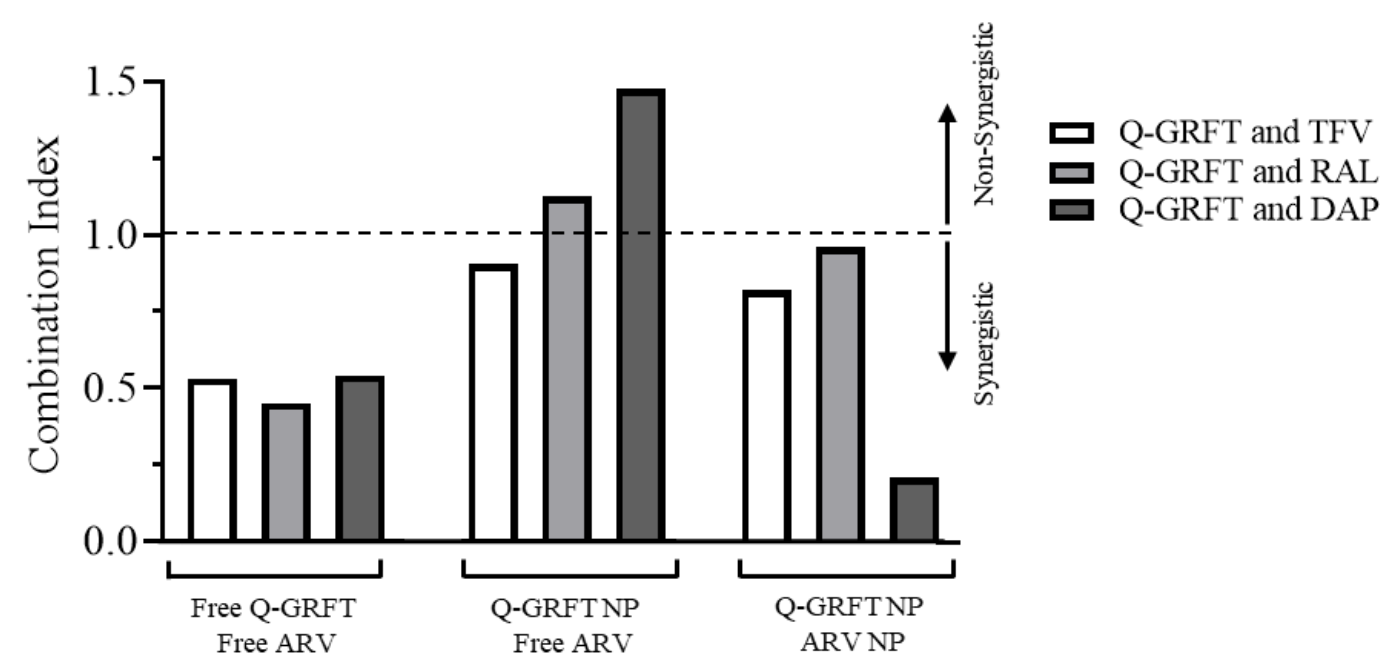

Figure 2.7. Combination indices of free Q-GRFT and free ARVs, Q-GRFT NPs and free ARVs, and Q-GRFT NPs and ARV NPs co-administered to TZM-bl cells.

Combinations of Q-GRFT with each ARV. When Q-GRFT was coadministered with free TFV, RAL, or DAP, the corresponding $\mathrm{Cl}$ values were 0.53 , 0.45 , and 0.54 , indicating similarly strong synergistic interactions between all protein-drug combinations.

Combinations of Q-GRFT NPs with each ARV. When Q-GRFT NPs were administered in combination with free TFV, RAL, or DAP, the calculated $\mathrm{Cl}$ values were $0.9,1.12$, and 1.47 showing synergistic interactions between Q-GRFT NPs and TFV.

Combinations of Q-GRFT NPs with ARV NPs. Co-administration of QGRFT NPs with ARV NPs demonstrated strong synergistic interactions with DAP NPs $(\mathrm{Cl}=0.21)$ and more modest synergistic interactions with TFV NPs and RAL NPs $(\mathrm{Cl}=0.82$ and 0.96 , respectively). Among the different combined treatment 
groups, the strongest synergistic interaction was observed for Q-GRFT NPs when administered with DAP NPs $(\mathrm{Cl}=0.21)$.

\section{Assessment of NP-Fiber Composites}

Based on the most synergistic formulation evaluated above, nanoparticles containing $10 \%$ of either Q-GRFT or DAP were successfully incorporated into the PEO portion of multilayered fibers to demonstrate the release of active agents over a longer duration. Q-GRFT and DAP were added in a ratio of 1:10 w:w NP:PEO fiber ( $1 \mathrm{mg} \mathrm{NP}: 10 \mathrm{mg}$ fiber) to obtain a final concentration of $10 \mu \mathrm{g}$ active agent $/ \mathrm{mg}$ NP-fiber composite (based on $100 \mu \mathrm{g}$ active agent/mg NP and $100 \mu \mathrm{g} \mathrm{NP/mg} \mathrm{PEO}$ fiber loading). The actual loading of Q-GRFT and DAP in the NP-fiber composite was $6.9 \pm 1.9$ and $7.1 \pm 0.46 \mu \mathrm{g} / \mathrm{mg}$, respectively. As shown in Figure 2.8, DAP was fully released within the first few days, with negligible release over 3 months. For Q-GRFT NP-fiber composites, a burst release of $0.68 \mu \mathrm{g}$ Q-GRFT/mg PEO was observed in the first day, followed by minimal release over 42 days. A second phase of release was observed between days 42 to 63 , followed by a gradual release for up to 90 days. The release values from most of the time points, for a 5 mg fiber-composite piece was within the in vitro $\mathrm{IC}_{50}$ range of free Q-GRFT and DAP (57 and $0.03 \mathrm{ng} / \mathrm{mL}$, in TZM-bl cells), with the exception of the 8 and $72 \mathrm{hr}$

time points for Q-GRFT ( 30 and $16 \mathrm{ng} / \mathrm{mL}$ ) and $14 \mathrm{~d}, 63$ to $84 \mathrm{~d}$, in which the release was below the detection limit $(78 \mathrm{ng} / \mathrm{mL})$. 

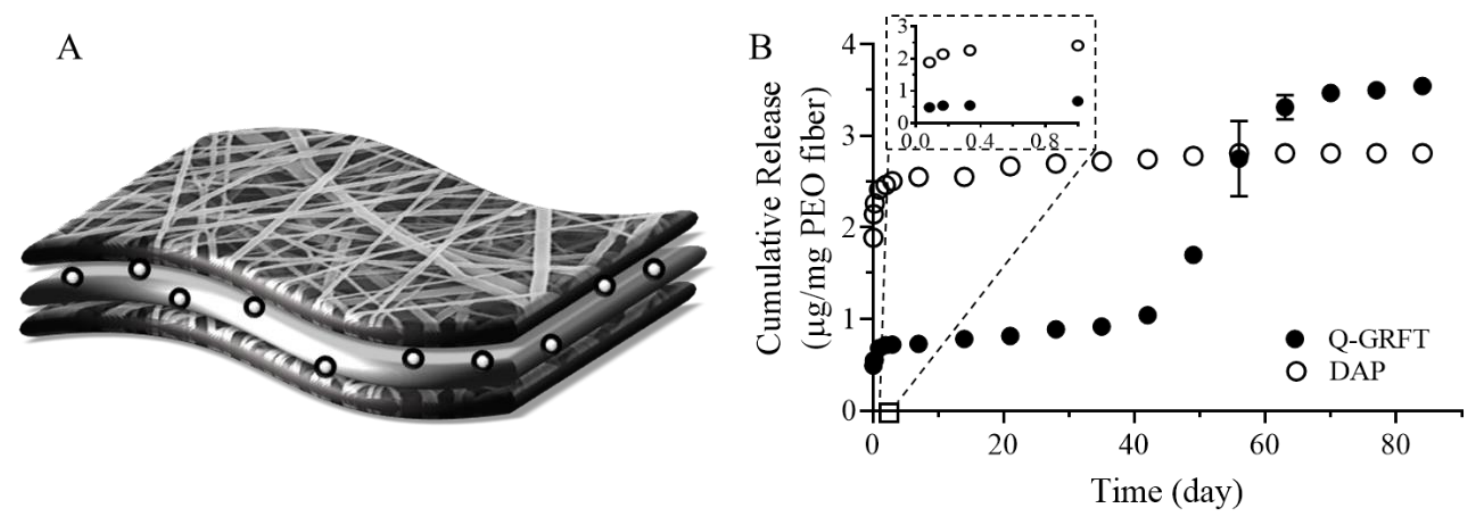

Figure 2.8. (A) Schematic of NP-fiber composites in which spheres may depict QGRFT NPs or DAP NPs. (B) The cumulative release of Q-GRFT and DAP from NP-fiber composites with an initial theoretical loading of $10 \mu \mathrm{g}$ active agent per $\mathrm{mg}$ PEO fiber. Release values are shown as the mean \pm standard deviation of three independent NP-fiber batches.

\section{In Vitro Cytotoxicity Studies}

Blank NPs, as well as Q-GRFT and ARV NPs were evaluated for cytotoxicity at the highest concentrations used for cell inhibition assays $(1 \mathrm{mg} / \mathrm{mL})$. No significant decreases in viability were observed in the viability of VK2, Ect1, and End1/E6E7 cell lines, after treatment with Q-GRFT, TFV, or RAL NPs, relative to untreated cells. These groups showed greater than $92 \%$ viability after 24,48 , and 72 hr NP administration; however, a significant decrease in cell viability was observed in VK2 cells treated with $1 \mathrm{mg} / \mathrm{mL}$ DAP NPs after 48 and $72 \mathrm{hr}(p \leq 0.01)$. In contrast, the negative control for viability (10\% DMSO) resulted in dramatically decreased cell viability spanning 7 to $18 \%$ across different cell lines (Figure 2.9, p $\leq 0.05)$. 


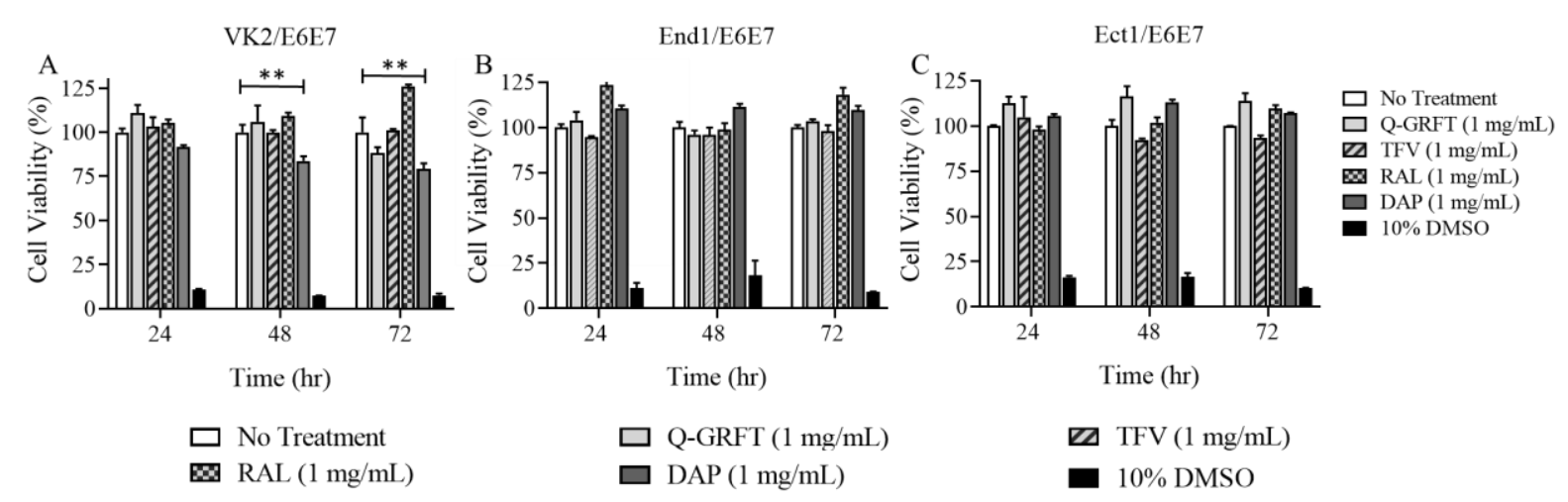

Figure 2.9. In vitro cytotoxicity of NPs encapsulating different agents administered to (A) VK2/E6E7 (B) End1/E6E7, and (C) Ect1/E6E7 cell lines using MTT assay. Viabilities are shown as the mean \pm standard deviation from administration of three independent samples. Statistical significance between experimental groups, as calculated by one-way ANOVA, is represented by ${ }^{* *} p \leq 0.01$.

\section{Discussion}

The co-administration of multiple active agents offers the potential to address some of the challenges surrounding PrEP, including low user adherence, high toxicity, and modest efficaciousness, by enabling virus targeting via different mechanisms of action and a corresponding decrease in the required dose and adaptation to drug resistance. Due to these benefits, synergistic interactions between a variety of ARVs have been studied to evaluate the effectiveness of a multi-agent approach to treat STIs such as HIV-1222,262,285-287. While the efficacy of TFV, RAL, or DAP in combination with other active agents including lamivudine, emtricitabine, and maraviroc has been investigated in clinical trials ${ }^{288-290}$, the combination of these ARVs with biologics, such as Q-GRFT, has been lesser explored $^{286}$. To date, there are only two studies that have evaluated the potential 
synergy between GRFT, a potent antiviral biologic, and ARVs for HIV-1 prevention $^{222,286}$. Furthermore, a new oxidation-resistant variant of GRFT (QGRFT) was developed which has not been investigated in combination with other active agents. Lastly, only one study has evaluated the impact of wild-type GRFT (relative to Q-GRFT) and ARV NP co-administration. Therefore, the goal of this study was to evaluate the synergy between the free and encapsulated forms of QGRFT and a few key "model" ARVs, which are used in oral PrEP and span different hydrophilicities and mechanisms of action.

While most ARVs inhibit virus post-cell entry, Q-GRFT is a biologic entry inhibitor with the potential to inhibit virus at the source of infection, prior to mucus penetration and cell entry. Due to the different mechanisms of action and the potential for early intervention with Q-GRFT, co-administration of Q-GRFT with established ARVs may enhance prophylactic and therapeutic outcomes.

Yet despite the promise of Q-GRFT and other antiviral agents, challenges such as the lack of consistent user adherence - often requiring frequent administrations and high dosing - and inadequate transport to target tissue, limit the utility of these agents in free form in clinical translation. To address these challenges, different dosage forms have been used to provide combinational delivery of these agents for HIV treatment. As two examples, polymeric films and IVRs have been used to deliver combinations of different active agents including TFV, DAP, and maraviroc ${ }^{288,291}$ for rapid versus sustained-delivery applications. For applications requiring delivery to physiologically complex environments, such as the FRT, polymeric NPs have been shown to dramatically enhance the transport of biologics 
and other active agents, and to enhance agent instability and cellular internalization 268,270,292. A recent study in our lab showed that PLGA NPs can encapsulate GRFT with high loading and showed promising efficacy against HIV1 in vitro and HSV-2 in vivo ${ }^{273}$. A recent study external to our group ${ }^{286}$, evaluated the synergy between GRFT and DAP in free, encapsulated, and co-encapsulated forms, demonstrating strong synergy between GRFT and DAP co-encapsulated in PLGA NPs at an equipotent IC 50 value ratio, against HIV-1286. To expand our knowledge to a variety of ARVs and to obtain a better understanding of synergistic interactions between active agent in free and encapsulated forms, we sought to evaluate the synergistic interactions between an oxidation-resistant variant of GRFT (Q-GRFT) and three different ARVs co-administered in free form, free and NP form, or NP form.

In this study, individual active agent encapsulation efficacy in NPs varied between 15 to $100 \%$, based on the properties of the encapsulated agent. Overall, NPs that encapsulated Q-GRFT, RAL, or DAP obtained encapsulation efficiencies higher than 49\%, while NPs provided lower loading (15\%) of the hydrophilic ARV, TFV (Table 2.1). Similar observations have been reported in previous studies, demonstrating only $16 \%$ TFV loading in PLGA NPs ${ }^{266}$. In contrast with TFV, QGRFT NPs had relatively high loading (49\%), which was similar to other studies that reported 41 and $42 \%$ loading of wild-type GRFT in PLGA NPs ${ }^{273,286}$, indicating similar loading potential for GRFT and Q-GRFT. This high level of loading may be attributed to the hydrophilicity of Q-GRFT and potential interactions of the mannose-binding sites which may interact via polar, electrostatic, or hydrogen 
bonding with hydrophobic residues of the PLGA matrix. In addition, protein surface absorption on polymeric NPs may also contribute to the higher loading observed with Q-GRFT, which may be further enhanced in future work by optimizing the concentration of protein and the use of stabilizers ${ }^{293}$. Interestingly, and despite the hydrophilicity and smaller size of RAL (e.g., relative to Q-GRFT), in our formulations, RAL obtained the highest loading, which was significantly higher than previously reported $(55 \%)^{287}$. Similarly, hydrophobic DAP achieved high NP encapsulation, which was in agreement with other studies that reported $70 \%$ loading of DAP in PLGA NPs. The high loading levels observed with DAP may be attributed to the efficiency with which the double emulsion technique encapsulates hydrophobic molecules ${ }^{286}$.

In addition to evaluating active agent loading, the release of each active agent from PLGA NPs was evaluated over 2 wk. Release results showed that all NP formulations provided modest and similar trends in sustained-release over the course of two weeks in SVF ( $<20 \%$, Figure 2.2). For Q-GRFT specifically, $11 \%$ of the total loading was released after $7 \mathrm{~d}$, which is similar to that reported for similarly loaded wild-type GRFT from PLGA NPs $\left(11 \%^{286}\right.$ and $\left.20 \%{ }^{273}\right)$. For TFV NPs, $~ 17 \%$ release was achieved over $14 \mathrm{~d}$, similar in amount released, to that observed under acidic $\mathrm{pH}$ conditions in another study ${ }^{266}$. Due to the relatively low loading and similarly low release of TFV observed from blended PLGA NPs in other work, formulations have moved to incorporate a more hydrophobic prodrug of TFV, tenofovir disoproxil fumarate, to obtain more desirable loading ( $57 \%)$, and increased release $(\sim 80 \% \text { release after } 48 \mathrm{hr})^{294}$. For RAL NPs, similar release 
( 11\%) to Q-GRFT was achieved, based on total loading; however, the release was lower than a previous report which showed $\sim 60 \%$ release after $7 \mathrm{~d}$ from PLGA NPs (in PBS) with similar $100 \mu \mathrm{g} / \mathrm{mg}$ theoretical loading ${ }^{234}$. Similarly, in our study, DAP release (16\%) was lower than previously reported results $(69 \%$ and $65 \%^{286,295}$ ); however, the theoretical loading was not reported, which is known to have a significant impact on release. Overall, similar ranges of release, as a function of overall loading, were observed for the active agents, which may be attributed to active agent entrapment in the NP, due to non-covalent binding between the agent and polymer. However, RAL and DAP NPs surprisingly exhibited higher levels of release as a function of mass active agent, which was unexpected due to their hydrophobicity. Previous studies have illustrated that encapsulant-polymer interactions and compatibilities have a significant impact on the particle structure, loading, and release characteristics ${ }^{296}$. In future work, these interactions may be modulated by integrating different materials and processing parameters such as polymer and drug concentration, polymer molecular weight, aqueous and organic phase volume, and drug content ${ }^{297}$. In addition, assessing the release of agents from NPs under more basic $\mathrm{pH}$ conditions is also recommended due to the increase in vaginal $\mathrm{pH}$ in the presence of semen. $\mathrm{A}$ previous study showed that GRFT release from PLGA NPs increased in PBS $(\mathrm{pH}=$ 7.4), relative to SVF ( $\mathrm{pH}=4.5$ ), while less DAP released from PLGA NPs in more basic $\mathrm{pH}$ conditions ${ }^{286}$.

Prior to conducting synergy studies, the $\mathrm{IC}_{50}$ of each individual free agent against HIV-1 in vitro, was determined relative to each corresponding NP- 
encapsulated agent (Figure 2.3). The $\mathrm{IC}_{50}$ of free Q-GRFT $(57.2 \mathrm{ng} / \mathrm{mL}, 4.5 \mathrm{nM})$ was slightly higher than the values for GRFT reported in previous studies (24 and $0.51 \mathrm{ng} / \mathrm{mL}$ ), when virus was added either $1 \mathrm{hr}$ post-incubation ${ }^{256}$ or simultaneous to treatment of TZM-bl cells ${ }^{286}$. In comparison, the $\mathrm{IC}_{50}$ S of free TFV and RAL in this study were $1.3 \mu \mathrm{M}(382 \mathrm{ng} / \mathrm{mL})$ and $7.42 \mathrm{nM}(3.3 \mathrm{ng} / \mathrm{mL})$ which were similar to previous studies (517 $\mathrm{nM}^{262}$ and $1.5 \mathrm{nM}^{234}$ for TFV and RAL, respectively) using the same cell line and administration time. For DAP, an $\mathrm{IC}_{50}$ of $0.1 \mathrm{nM}(0.034$ $\mathrm{ng} / \mathrm{mL}$ ) was observed here, which was lower than previous reports of $4.7 \mathrm{nM}$. However, the higher $\mathrm{IC}_{50}$ value may be attributed to differences in treatment regimen. In our study, cells were infected with the virus $1 \mathrm{hr}$ post-administration, while in previous work, TZM-bl cells were infected with the virus immediately after drug administration. In addition, it has been shown that the virus subtype impacts the $\mathrm{IC}_{50}$ of DAP (and other active agents), and based on this, these values can vary between 0.8 to $3.5 \mathrm{nM}^{298}$. When these active agents were encapsulated in NPs, similar $\mathrm{IC}_{50}$ values to those with free agent administration were observed, with the exception of Q-GRFT NPs in which the $\mathrm{IC}_{50}$ was 4.1 -fold lower than observed with free $\mathrm{Q}-\mathrm{GRFT}$. For Q-GRFT NPs, the lower $\mathrm{IC}_{50}$ values are in agreement with another study that showed the $\mathrm{IC}_{50}$ of wild-type GRFT in PLGA NPs decreases, relative to free $\mathrm{GRFT}^{273}$, possibly by enhancing protein stability and activity.

To evaluate the potential of Q-GRFT and ARV combinations to synergistically prevent HIV-1 infection, free Q-GRFT was co-administered with free ARVs, Q-GRFT NPs with free ARVs, and Q-GRFT NPs with ARV NPs, and the 
$I_{50}$ values of the individual agents were compared to their combinations in each form (Table 2.3). When free Q-GRFT was co-administered with each free ARV, synergistic interactions were observed for all co-administration groups with a 3.7 to 6.3-fold decrease in the $\mathrm{IC}_{50}$ of free Q-GRFT and a 2.8 to 5.6 -fold reduction in the $I_{50}$ values of free ARVs (Figure 2.4). These results are in agreement with previous studies that assessed the impact of co-administering equipotent ratios (1:1 $\mathrm{IC}_{50}$ ratios) of free GRFT and TFV $(\mathrm{Cl}=0.6)$ in CD4+ MT-4 cells ${ }^{222}$. Another study demonstrated very strong synergy $(\mathrm{Cl}<0.1)$ between free GRFT and free DAP co-administered to TZM-bl cells in equipotent ratios ${ }^{286}$.

More interestingly, when Q-GRFT and ARVs were each encapsulated in NPs, the synergistic interactions observed between Q-GRFT and DAP incorporated in NPs significantly improved relative to free Q-GRFT and free DAP co-administration (Figure 2.6). In fact, among all of the co-administration groups, the most synergistic interaction was observed between Q-GRFT NPs and DAP NPs with 6.0 and 8.6-fold improvements in activity for Q-GRFT and DAP, respectively. This observation is in agreement with a previous study that showed strong synergy between GRFT NPs and DAP NPs $(\mathrm{Cl}=0.08)$ using TZM-bl cells and equipotent active agent ratios ${ }^{286}$. The lower synergy observed in our study, between Q-GRFT NPs and TFV NPs or Q-GRFT NPs and RAL NPs may be due to the higher $\mathrm{IC}_{50}$ value for each $\mathrm{ARV}$, which requires higher drug concentrations to be released from NPs to exert efficacy. Co-administration of active agents with different mechanisms of action in NPs may increase prophylactic effect, decrease the dose needed to attain efficacy against HIV-1 infection, and reduce the chance 
of developing drug resistance. Furthermore, previous work has shown that encapsulating active agents in NPs may improve synergistic interactions by enhancing drug uptake and cell internalization ${ }^{262}$. Moreover, NPs may overcome chemical incompatibilities between co-administered agents ${ }^{262}$. While synergy was observed in all NP formulations, in future studies, different molar ratios of Q-GRFT NPs:ARV NPs can be explored to seek the most synergistic ratios. Moreover, the administered ratios may be adjusted based on the toxicity of each agent to balance toxicity with efficacy. In addition, the time frame of agent administration prior to virus exposure may be of interest to investigate. Since each active agent has different mechanism of action and inhibits the virus at different stages of infection, administration time can play a role in obtaining efficacy and synergistic interactions between agents.

In addition to free Q-GRFT/free ARV and Q-GRFT NP/ARV NP coadministration, the efficacy of co-administration of Q-GRFT NPs with free ARVs was evaluated. Overall, no reduction in the $\mathrm{IC}_{50}$ values of free DAP was observed; however, there was a modest decrease of 1.6 and 1.4 -fold in the $\mathrm{IC}_{50}$ values of free TFV and free RAL (Figure 2.5). Antagonistic interactions between Q-GRFT NPs and free RAL as well as Q-GRFT NPs and free DAP $(\mathrm{Cl}>1)$ may be attributed to the unfavorable electrostatic interactions between PLGA NPs and the free drug due to the negative charge of PLGA NPs. Moreover, the high protein binding affinity of PLGA NPs with proteins in the cell media may affect the efficacy of active agents. In addition, the lower release of Q-GRFT, relative to available free TFV, RAL, and DAP, may not provide the most synergistic ratio. 
In addition to efficacy, the safety of NPs encapsulating each of the active agents was evaluated in vitro, and showed that encapsulated Q-GRFT, TFV, RAL, and DAP at the maximum dose used in efficacy studies $(1 \mathrm{mg} / \mathrm{mL})$, are non-toxic to vaginal cells. Previous studies have also demonstrated the safety of PLGA NPs loaded with GRFT ${ }^{273}, \mathrm{TFV}^{267}, \mathrm{RAL}^{287}$, and $\mathrm{DAP}^{286,295}$ in vitro and in vivo 273,286 further validating that co-administration can enhance the efficacy of these active agents while maintaining their safety. It should be mentioned that the relatively higher toxicity of DAP NPs in VK2/E6E7 cells may be mitigated with the administration of lower doses via synergistic and targeted NP delivery. Moreover, due to the high synergy between Q-GRFT NPs and DAP NPs, a lower dose of active agent may be considered to obtain strong efficacy against HIV-1.

In line with promising efficacy and safety, another important criterion is to provide a practical long-term prevention strategy in an effective delivery platform. The NP co-administration strategy evaluated here inhibited HIV-1 infection in vitro, and with one exception (i.e., TFV), released efficacious concentrations within the IC 50 range of each active agent in TZM-bl cells, for up to 7 days. However, this release may be improved, by either improving the NP formulation, or creating a hybrid delivery platform similar to previous work ${ }^{299}$. In this study, we preliminarily evaluated NP-fiber composites to prolong the release of our most synergistic NP formulations, by incorporating Q-GRFT NPs and DAP NPs. In our NP-fiber composite platform, DAP, a small hydrophobic molecule, showed a burst release followed by a minimal release that was still in the $\mathrm{IC}_{50}$ range of DAP observed in TZM-bl cells. On the other hand, the Q-GRFT release curve followed an "S" shape, 
indicating two distinct release phases on day 1 and days 42 through 60, each of which was followed by more gradual release. We attribute DAP release within the first few hours to the small size of DAP, while Q-GRFT, due to its larger size, may have been entrapped within fiber layers, providing slightly more gradual release through day 42. The second phase of release is attributed to NP-fiber polymer degradation, resulting in another burst release. These release profiles are in agreement with our previous work with that incorporated wild-type GRFT in a NPfiber composite ${ }^{273}$. Furthermore, the release profile may be optimized by changing the NP content and altering the outer layer thickness ${ }^{273}$.

In addition to modulating the release, NP-fiber composites like NPs alone, may also enable virus inhibition at different stages by providing a burst release (here of DAP and NNRTI), which can provide an on-demand rapid protection against HIV-1, followed by more prolonged release of Q-GRFT, an entry inhibitor, for long-term prevention and treatment. In addition to virus inhibition via the use of active agents, previous studies in our group have shown that the electrospun fibers can also physically trap the virus, which may lend increased protection through physical interactions ${ }^{128}$. Moreover, lower doses of active agents are released from these platforms which may result in less toxicity. In the future, NP-fiber composites may serve as a multipurpose delivery platform to target multiple infection types by encapsulating different agents and may enhance NP retention relative to free NP administration, to promote user adherence if release can be tuned for specific agents. 


\section{Conclusion}

In this work, NPs encapsulating Q-GRFT, TFV, RAL, and DAP were successfully developed with high loading and efficacy against HIV-1 pseudovirus infection in vitro. In addition, the synergistic interactions between free Q-GRFT and free ARVs, Q-GRFT NPs and free ARVs, and Q-GRFT NPs and ARV NPs were assessed. Significant enhancements in efficacy were observed when free Q-GRFT and free ARVs were co-administered, relative to the administration of individual active agents. Moreover, synergy was observed between free Q-GRFT and all ARVs when they were individually encapsulated in PLGA NPs. For Q-GRFT and DAP in particular, synergistic interactions improved upon co-administration of QGRFT NPs with DAP NPs. Overall, this work shows that the potent HIV-1 inhibitor, Q-GRFT, can be co-administered with different ARVs, including NRTIs and integrase inhibitors, to improve antiviral efficacy against HIV-1, and that modest, therapeutically-relevant sustained-release can be achieved over 14 days. Furthermore, the co-administration of Q-GRFT with ARVs may be further considered in future studies to impart long-term protection by modulating multiagent release in hybrid platforms such as NP-fiber composites. 


\section{CHAPTER 3}

\section{A COMPUTATIONAL STUDY OF INTERACTIONS BETWEEN ANTIRETROVIRALS AND PLGA NANOPARTICLES}

\section{Introduction}

The human immunodeficiency virus (HIV) is one of the most serious viral infections worldwide, impacting more than 37 million people ${ }^{300}$. Antiretrovirals (ARVs) have been used to treat HIV-1 by inhibiting virus replication at different stages of the viral life cycle ${ }^{213}$. These active agents are classified into five different groups based upon their mechanism of action: 1 . Nucleoside reverse transcriptase inhibitors (NRTIs); 2. Non-nucleoside reverse transcriptase inhibitors (NNRTIs); 3. Protease inhibitors (PIs); 4. Integrase inhibitors (INI); and 5. Co-receptor inhibitors (CRI). TFV, RAL, and DAP are a few examples of thirty approved ARVs that have been widely used for HIV-1 treatment. Tenofovir is a NRTI that is phosphorylated in situ to obtain its virologically active form, tenofovir diphosphate. Tenofovir diphosphate prevents both HIV-1 and HIV-2 DNA polymerases from integrating into viral DNA by competing against the nucleotide deoxyadenosine 5'triphosphate. After integration, tenofovir diphosphate terminates DNA synthesis due to the absence of a ribose ring, a moiety responsible for elongating a proviral DNA strand ${ }^{301}$. RAL is an INI that inhibits the integration of HIV-1 DNA into the host genome by preventing the formation of covalent bonds between integrase, an 
enzyme responsible for the transfer of viral DNA into the host chromosome and the host DNA ${ }^{218,302}$. Moreover, the fluorine atom in RAL is strongly electronegative and can interact with $\mathrm{H}$-atom donors in the enzyme as well as contributing to an enhancement of the inhibitor lipophilicity that improves cell permeability ${ }^{303}$. Dapivirine (DAP), an NNRTI, is a substituted diarylpyrimidine derivative that tightly binds to HIV reverse transcriptase and prevents virus replication ${ }^{304}$.

Antiretrovirals can be delivered via different modalities that include oral and topical pre-exposure prophylaxis (PrEP) and post-exposure prophylaxis (PEP). Oral delivery of ARVs is challenged with serious adverse effects such as bone marrow suppression and toxic hematologic side effects ${ }^{220}$ and has been shown to cause virus mutations ${ }^{221}$. These harmful effects are often exacerbated by the poor oral bioavailability and frequent dosing required by many of these drugs. As an alternative, topical, intravaginal, or intrarectal administration of ARVs using different delivery platforms has been used to address these challenges and have shown promise in HIV-1 treatment. In particular, polymeric NPs have been widely used to encapsulate different agents since they can maintain agent stability, improve cell penetration, and provide sustained release of the active agents $^{295,305,306}$. PLGA is one of the most widely used polymers for ARV delivery due to its biocompatibility and biodegradation properties. Previous studies have shown that using PLGA NPs can increase the permeability and bioavailability of the agents ${ }^{307}$.

Despite the potential importance of drug and polymer interactions in developing effective delivery vehicles, there are only a few studies that have used 
computational chemistry or molecular dynamics (MD) to investigate interactions between drugs and their delivery platforms ${ }^{308-313}$. One study has used MD simulations to predict the compatibility of two hydrophobic drugs in poly(ethylene oxide)-b-poly( $\varepsilon$-caprolactone) (PEO-b-PCL) block copolymer micelles with different molecular weights ${ }^{308}$. Flory-Huggins interaction parameters calculated for different drug-polymer systems in this study have shown that this parameter is not sensitive to the hydrophilic PEO chain length but strongly depends on the hydrophobic chain length of $P C L^{308}$. In a similar study, the drug loading capacity of PEO-b-PCL copolymer for two anticancer hydrophobic drugs was assessed utilizing the Flory-Huggins interaction parameter and radial distribution function (RDF). Findings were compared to in vitro drug release from PEO- $b$-PCL micelles ${ }^{309}$. This study demonstrated that an increase in the PCL/PEO $(w / w)$ ratio increases the solubility of hydrophobic drugs due to the multiple hydrogen bonding sites on the PCL block that may interact with hydrogen bonding sites on the drug molecule ${ }^{309}$. Another study regarding drug and polymer interactions focused on the analysis of intermolecular interactions between hydrophobic drugs and PEO$b$-PCL block copolymers to evaluate the encapsulation capability of multihydrophobic block structures ${ }^{310}$. The results showed that using three PCL blocks can significantly enhance the drug-polymer interactions by increasing the number of hydrogen bond donors and acceptors ${ }^{310}$. An additional study on this subject utilized MD simulations to characterize interactions between an insoluble drug and PEG-poly(hexyl-substituted poly(lactide) micelles ${ }^{311}$. Solubility parameters resulting from MD simulations in that study were in agreement with experimental 
results, indicating that simulation methods can provide an insight into the encapsulation capabilities of delivery platforms ${ }^{311}$. Another study used MD simulations and docking calculations to develop a model to predict the loading of different agents in polymeric nanospheres ${ }^{313}$. This model was established using the binding energies of different agents including curcumin, paclitaxel, and vitamin D3 in an ABA-triblock nanosphere comprised of poly(ethylene glycol) (A blocks) and tyrosine-derived polyacrylate $(\mathrm{B} \text { block })^{313}$.

While there is no study on simulating the intermolecular interactions between polymeric delivery platform and anti-HIV agents, there is one study that used MD simulation to investigate the interactions between water-soluble anionic carbosilane dendrimers and primary proteins of HIV-1 such as $\mathrm{gp} 120^{312}$. That study showed that gp120 and the dendrimer make a complex with high binding affinity which inhibits the virus binding to the cell receptor and results in viral inactivation.

Some of the challenges in developing nanoparticle formulations involve the successful encapsulation of different active agents with diverse properties including molecular weight, hydrophobicity, size, and drug-polymer compatibility. These challenges may limit the choice of polymers and drugs for encapsulation purposes. Simulation methods, however, can help to reduce the time required to identify the best candidate among countless number of polymer formulations for a specific active agent ranging from small drug molecules to peptides and large proteins. Given the importance of simulations in optimizing experiments and predicting experimental results, several studies have reported different 
encapsulation efficiencies for PLGA NPs encapsulation of different ARVs that can depend on drug hydrophobicity, size, and other physical and structural factors $^{266,287,295}$. A better understanding of the polymer and active agents can help to explain and predict experimental results and improve the drug-delivery systems. To provide insight into the ARV encapsulation of PLGA NPs, molecular mechanics and MD simulations are applied in the current study to investigate the interactions between different $A R V s$ and their polymeric delivery platforms. The objective of this study is to investigate the importance of hydrogen bonding between the polymer and different drug molecules on the loading capacity of PLGA NPs.

\section{Experimental Design}

\section{Materials}

Materials. Poly(vinyl alcohol) (PVA, 87-90\% hydrolyzed, 30000-70000 MW) and PLGA used to synthesize NPs (lactic acid:glycolic acid 50:50, viscosity: 0.55-0.75 dL/g, 31000-57000 MW) were purchased from Sigma Aldrich (St. Louis, MO) and Lactel Absorbable Polymers (Cupertino, CA), respectively. Tenofovir (TFV) and Dapivirine (DAP) were obtained from Sigma Aldrich (St. Louis, MO) while Raltegravir (RAL) was provided by Selleckchem (Houston, TX). Dichloromethane (DCM) was purchased from Thermo Fisher (Waltham, MA). Other chemicals including dimethyl sulfoxide (DMSO) were acquired from Sigma Aldrich.

NP Synthesis. Blank NPs and NPs loaded with TFV, RAL, or DAP were fabricated using either single or double emulsion techniques. These drugs were 
selected as model molecules because of their different hydrophilicity. For each formulation, $100 \mathrm{mg}$ of PLGA was dissolved in $2 \mathrm{~mL}$ of $\mathrm{DCM}$ and incubated overnight. For TFV and RAL NPs, $10 \mathrm{mg}$ of the active agent was dissolved in 200 $\mu \mathrm{L}$ of Tris-EDTA (TE) buffer. For DAP NPs, $10 \mathrm{mg}$ of DAP was dissolved in 200 $\mu \mathrm{L}$ of DCM. The active agent solution was added dropwise to the polymer solution while the solution was vortexed. Then, the active agent-polymer solution was sonicated and added to 5\% PVA in Milli-Q water (2 mL), vortexed, and sonicated again. The emulsified solution was then added to a larger volume of aqueous solution ( $50 \mathrm{~mL}$ of $0.3 \%$ PVA) and incubated for $3 \mathrm{hr}$ with stirring in order to evaporate DCM. Next, NPs were centrifuged and pelleted $(25,364 \mathrm{xg}$ for $10 \mathrm{~min}$ at $4^{\circ} \mathrm{C}$ ), washed three times using $30 \mathrm{~mL}$ Milli-Q water, resuspended in $5 \mathrm{~mL}$ Milli$\mathrm{Q}$ water, and incubated at $-80^{\circ} \mathrm{C}$ for $2 \mathrm{hr}$. Finally, NPs were lyophilized to obtain solid particles and stored at $-20^{\circ} \mathrm{C}$.

Size. The size and morphology of unhydrated NPs were determined using scanning electron microscopy (SEM). First, samples were sputter-coated with a palladium/gold alloy layer of a thickness of 15-18 nm, and imaged using a Supra 35 SEM (Zeiss, Oberkochen, Germany). The average size of NPs was analyzed with Image J software (NIH, Bethesda, Maryland) by measuring the diameter of 50 elements per image and 3 images for each formulation.

Loading. To assess the loading of NPs, 1-2 mg of NPs was dissolved in $100 \mu \mathrm{L}$ of DMSO and diluted with TE buffer to the final volume of $1 \mathrm{~mL}$. Serial 1:2 sample dilutions were then made in TE buffer. To quantify the amount of TFV, $\mathrm{RAL}$, and DAP, plate absorbance was measured at 260,300 , and $290 \mathrm{~nm}$ on a 
Synergy HT reader (BioTek, Winooski, VT, USA). After assessing the loading, the encapsulation efficiency of each drug was calculated by dividing the weight of drug encapsulated in the NPs by the mass of drug added to the polymer solution during the synthesis.

\section{Simulation Method}

The Condensed-phase Optimized Molecular Potentials for Atomistic Simulations Studies (COMPASS) forcefield 314,315 was used for all atomistic simulations. This forcefield was developed using ab initio and empirical parametrization techniques and is especially parameterized for the simulation of polymers and small molecules. Gaussian 09 Software $^{316}$ was used for all computational chemistry calculations.

Cohesive Energy Density. First, water, a polymer chain (40 repeat units), and each drug molecule were built individually and the geometry was optimized using COMPASS forcefield. Next, an amorphous cell with periodic boundary conditions containing 100 drug molecules, or 5 polymer chains, was constructed

to achieve a cell size of $30-40 \AA$ on aside. The amorphous cell was then geometry optimized followed by 200-ps NVT molecular dynamics using the Nosé thermostat ${ }^{317}$ at a constant temperature of $310 \mathrm{~K}$ to mimic biological temperature. The final configuration was used for NPT calculations that were performed at 310 $\mathrm{K}$ and 1 bar using Nosé thermostat. The simulation time of 2 ns for NPT calculations was long enough for the system to equilibrate as measured by the unit cell size stabilization. The cohesive energy density (CED) was obtained by 
calculating the summation of bounded and non-bounded energies. The Hildebrand solubility parameter $(\delta)$ was calculated from the square root of the CED. For comparison, the CED of each drug was obtained from experimental results found in literature ${ }^{318,319}$ or calculated using a group-contribution method ${ }^{320}$. In this approach, each molecular structure is split into parameterized groups. The CED was obtained by summing all the group contributions. For water ${ }^{318}$ and PLGA ${ }^{319}$, solubility parameters were directly obtained from experimental values found in literature. The drug-polymer miscibility was then evaluated by comparing the solubility parameters of each drug and PLGA.

Radial Distribution Function (RDF). The radial distribution function provides the probability density $(g)$ for a specific atom to have another atom as a neighbor at a given distance and therefore is a useful tool to investigate atomistic interactions. If the value of $g_{\mathrm{OH}}$ between different pairs of interacting hydrogen and oxygen atoms, goH, is larger than unity, strong interactions between the two atoms are indicated. The positions of peaks in the RDF plot show the preferred interatomic distances between oxygen and hydrogen atoms. Thus, if the RDF for an oxygen and hydrogen of two separate molecules is smaller than their covalent radii (1.5-2.5 $\AA$ ), an interaction between atoms is evident. In our simulations, the amorphous cell containing either 15 drug molecules and 2000 water molecules or 1 polymer chain and 2000 water molecules was created to achieve the cell with desired size of $\sim 30-40 \AA$. This was followed by geometry optimization. The last 200 frames of the trajectory file from NPT calculations (1.8-2.0 ns) were used to obtain the RDF values. Hydrogen and oxygen atoms of each molecule were 
selected as a group and the intermolecular interactions between each forcefield type (OH and $\mathrm{HN}$ groups) were determined.

Computational Chemistry Calculations. Each drug molecule and PLGA were created in Gaussian and geometry optimized using density-functional theory (DFT) calculations (B3LYP/6-311G(d,p)) to determine atomic ESP charges. Then, the calculated charges were compared to the electrostatic point charges used in the MD force fields.

\section{Results and Discussion}

Nanoparticle Characterization. PLGA NPs encapsulating $100 \mu \mathrm{g}$ of each ARV per mg of NP, were synthesized with high yield (>70\%) and spherical morphology, are shown by SEM images provided in the Figure 3.1. Information on NPs size, loading, and encapsulation efficacy is given in Table 3.1. The size of the NPs ranged from 89.9 to $107.2 \mathrm{~nm}$. Loadings of $15.4 \pm 3.3,103.5 \pm 11.9$, and 60.2 $\pm 2.7 \mu \mathrm{g} / \mathrm{mg} \mathrm{NP}$ were obtained for TFV, RAL, and DAP NPs, respectively. These results show differences in encapsulation capabilities of PLGA NPs for different active agents. To obtain a better understanding of those experimental results, molecular dynamics simulations were used in this work to study the potential 
influence of drug and polymer intermolecular interactions on the level of drug encapsulation.
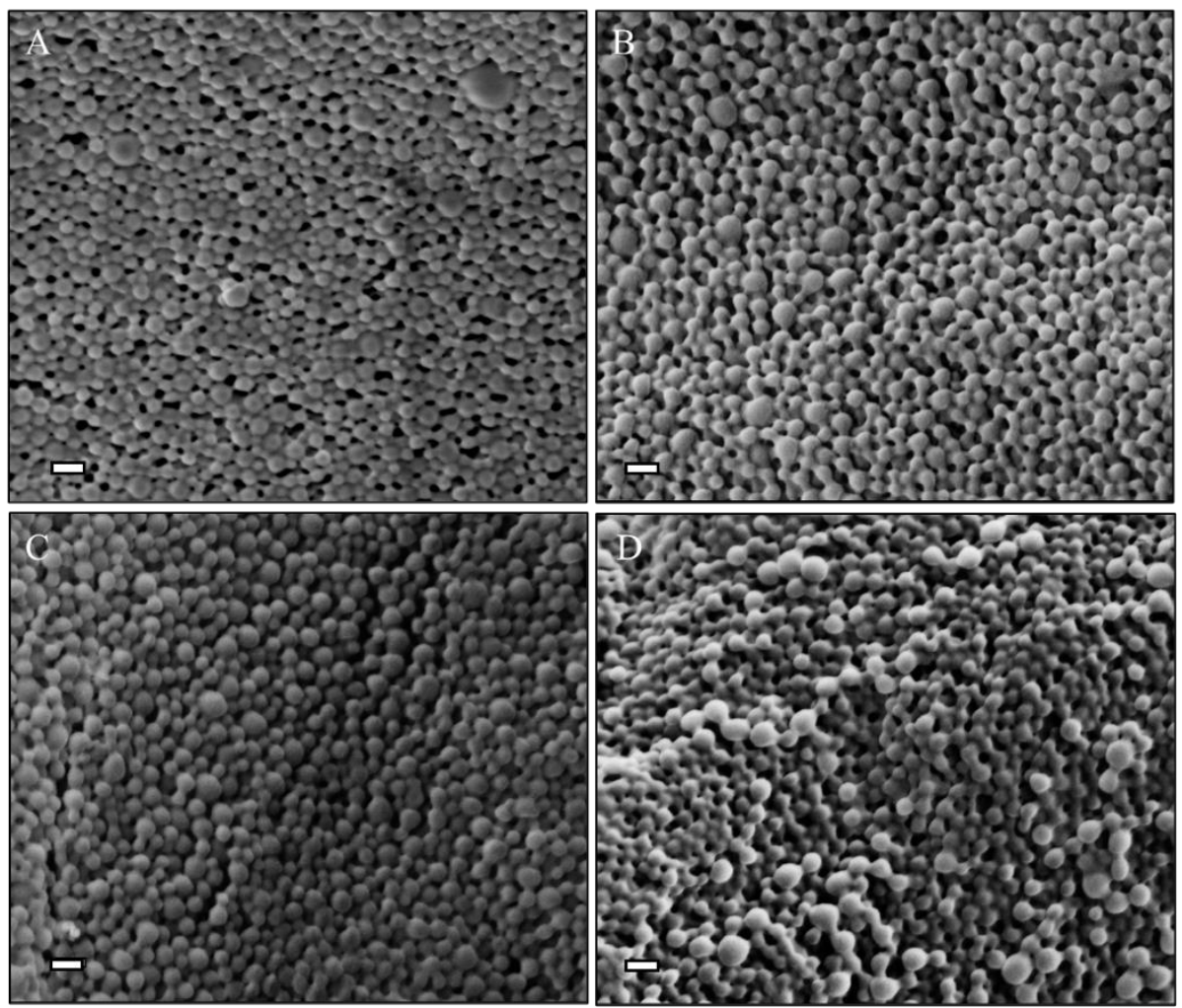

Figure 3.1. Scanning electron microscopy images of PLGA nanoparticles loaded with 10\% w/w (A) Q-GRFT, (B) TFV, (C) RAL, and (D) DAP. Scale bars represent $200 \mathrm{~nm}$.

Table 3.1. Size, loading, and encapsulation efficiency (\%) of PLGA NPs encapsulating different ARVs.

\begin{tabular}{|c|c|c|c|}
\hline Drug & Size (dia. $\mathbf{n m} \pm$ STD) & Loading $(\boldsymbol{\mu g} / \mathbf{m g})$ & Encapsulation Efficacy (\%) \\
\hline TFV & $89.9 \pm 25.3$ & $15.4 \pm 3.3$ & $15.4 \pm 3.3$ \\
\hline RAL & $106.3 \pm 29.7$ & $103.5 \pm 11.9$ & $103.5 \pm 11.9$ \\
\hline DAP & $107.2 \pm 27.1$ & $60.2 \pm 2.7$ & $60.2 \pm 2.7$ \\
\hline
\end{tabular}


Atomic Charge Distribution. The geometry-optimized structures for the ARVs and PLGA are shown in Figure 3.2. ESP Charges for each atom are shown in Table 3.2. Results indicated that although there are slight differences between charges obtained from molecular dynamics using the COMPASS forcefield and B3LYP/6-31 $(d, p)$ calculated from Gaussian, similar trends in charge distribution were observed. In the case of TFV, phosphorus (COMPASS forcefield type P4=) and nitrogen (n3h2) atoms connected to hydrogen atoms had the highest positive and negative partial atomic charges, respectively. The highest positive and negative atoms in RAL were hydrogen connected to the oxygen (h10) and nitrogen (n3mh and n3h1) atoms connecting to hydrogen. In the case of DAP, hydrogen atoms connected to the nitrogen and nitrogen ( $\mathrm{n} 1 \mathrm{t}$ and $\mathrm{n} 2 \mathrm{a}$ ) atoms connected to carbon atoms possessed highly positive and negative partial atomic charges. These atoms were specifically chosen to investigate electrostatic interactions between drug molecules and water or the drug molecules and the polymer. 


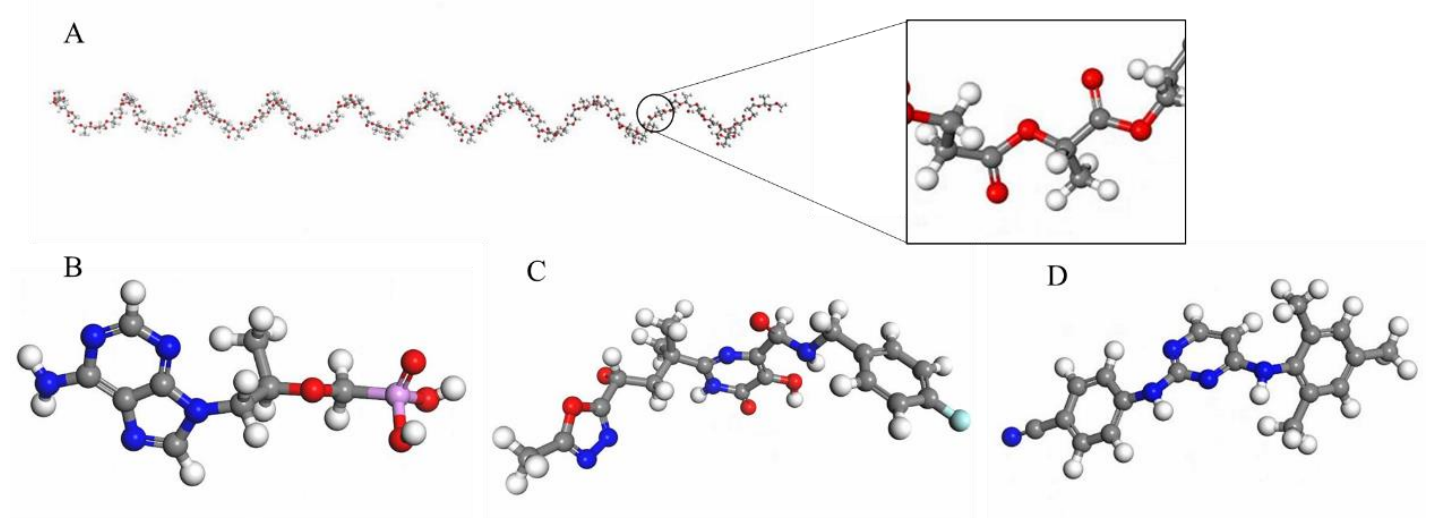

Figure 3.2. Geometry-optimized structure of A) PLGA, B) TFV, C) RAL, and D) DAP. Phosphorus, Fluoride, oxygen, nitrogen, carbon, and hydrogen atoms are shown in magenta, cyan, red, blue, gray, and white. 
Table 3.2. Atomic charges for TFV, RAL, and DAP.

\begin{tabular}{|c|c|c|c|c|}
\hline Atom & \begin{tabular}{|c|} 
COMPASS \\
Forcefield Type
\end{tabular} & $\begin{array}{l}\text { Charge (Materials } \\
\text { Studio, ESP) }\end{array}$ & $\begin{array}{c}\text { Charge } \\
\text { (Gaussian, ESP) }\end{array}$ & $\begin{array}{c}\text { Number of } \\
\text { Atoms }\end{array}$ \\
\hline \multicolumn{5}{|c|}{ Tevofovir } \\
\hline$P$ & $\mathrm{P} 4=$ & 0.82 & 1.14 & 1 \\
\hline \multirow{3}{*}{$\mathrm{O}$} & $02 \mathrm{e}$ & -0.32 & -0.42 & 1 \\
\hline & $\mathrm{o} 1=$ & -0.49 & -0.40 & 1 \\
\hline & $\mathrm{o} 2 \mathrm{e}$ & -0.55 & $-0.61 /-0.69$ & 2 \\
\hline \multirow{4}{*}{$\mathrm{H}$} & h1o & 0.41 & 0.40 & 2 \\
\hline & h1 & 0.05 & $0.01-0.10$ & 12 \\
\hline & h1 (ring) & 0.13 & $0.07-0.10$ & 2 \\
\hline & h1n & 0.35 & 0.34 & 2 \\
\hline \multirow{3}{*}{$\mathrm{N}$} & $\mathrm{n} 2 \mathrm{a}$ & -0.48 & $-0.51 /-0.79$ & 3 \\
\hline & $\mathrm{n} 3 \mathrm{a}$ & 0.00 & -0.46 & 1 \\
\hline & $\mathrm{n} 3 \mathrm{~h} 2$ & -0.72 & -0.70 & 1 \\
\hline \multicolumn{5}{|c|}{ Raltegravir } \\
\hline $\mathrm{F}$ & f1 & -0.13 & -0.25 & 1 \\
\hline \multirow{3}{*}{$\mathrm{O}$} & $\mathrm{o} 1=$ & -0.45 & $-0.30-(-0.58)$ & 3 \\
\hline & $\mathrm{o} 2 \mathrm{~h}$ & -0.44 & -0.57 & 1 \\
\hline & $\mathrm{o} 2 \mathrm{a}$ & -0.06 & -0.44 & 1 \\
\hline \multirow{4}{*}{$\mathrm{N}$} & $\mathrm{n} 2 \mathrm{a}$ & -0.24 & $-0.3 /-0.47$ & 2 \\
\hline & n3h1 & -0.64 & -0.44 & 1 \\
\hline & $\mathrm{n} 3 \mathrm{mh}$ & -0.57 & -0.85 & 1 \\
\hline & $\mathrm{n} 2=$ & -0.3 & -0.59 & 1 \\
\hline \multirow{5}{*}{$\mathrm{C}$} & c3' & 0.45 & $-0.3-(-0.58)$ & 3 \\
\hline & c4 & -0.16 & -0.45 & 7 \\
\hline & $\mathrm{c} 3 \mathrm{a}$ & 0.27 & $-0.01-(-0.41)$ & 7 \\
\hline & $\mathrm{c} 3=$ & 0.03 & $0.23 / 0.30$ & 2 \\
\hline & c3o & 0.48 & 0.38 & 1 \\
\hline \multirow{3}{*}{$\mathrm{H}$} & h1 & 0.127 & $0.09-0.15$ & 17 \\
\hline & h1o & 0.41 & 0.39 & 1 \\
\hline & h1n & 0.35 & $0.23 / 0.30$ & 3 \\
\hline \multicolumn{5}{|c|}{ Dapivirine } \\
\hline \multirow{3}{*}{$\mathrm{N}$} & $\mathrm{n} 1 \mathrm{t}$ & -0.43 & -0.71 & 1 \\
\hline & n3h1 & -0.37 & $-0.45 /-0.54$ & 2 \\
\hline & $\mathrm{n} 2 \mathrm{a}$ & -0.48 & -0.53 & 2 \\
\hline \multirow{4}{*}{$\mathrm{C}$} & $\mathrm{c} 3 \mathrm{a}$ & 0 & $0.01-0.53$ & 15 \\
\hline & $\mathrm{c} 2 \mathrm{t}$ & 0.22 & 0.75 & 1 \\
\hline & c3az & 0.21 & 0.4 & 1 \\
\hline & c4 & -0.16 & 0.97 & 3 \\
\hline \multirow{2}{*}{$\mathrm{H}$} & h1 & 0.05 & $0.09-0.12$ & 17 \\
\hline & h1n & 0.35 & 0.31 & 2 \\
\hline
\end{tabular}


Cohesive Energy Densities. In order to validate the COMPASS forcefield for water, PLGA, and drugs, the cohesive energy density (CED) for each compound was obtained using MD (COMPASS forcefield) calculations and compared to experimental values for water ${ }^{318}$ and $\mathrm{PLGA}^{319}$ in the literature or using a group-contribution method ${ }^{320}$. As shown in Table 3.3, the solubility parameters calculated for water, PLGA, TFV, RAL, and DAP were 44.87, 25.26, 26.25, 24.51, and 22.67 $\mathrm{MPa}^{1 / 2}$, respectively. The computed values were in reasonable agreement with those calculated using functional-group contributions and experimental values with $7 \%$ to $20 \%$ difference between two values. This is in agreement with previous studies that have shown that molecular simulations using COMPASS forcefield can satisfactorily predict the structural, conformational, and cohesive properties of polymers and drug molecules ${ }^{308,309}$. In addition, previous studies have demonstrated that if the difference in solubility parameters between two molecules is less than $7 \mathrm{MPa}^{1 / 2}$, they are likely to be miscible ${ }^{321}$. Given this, similar solubility parameters for TFV, RAL, DAP, and PLGA indicate that all of these ARVs and the polymer are thermodynamically miscible, suggesting that PLGA-drug blend is a physically stable dispersion, while experimental results showed low compatibility between TFV and PLGA. In addition, the large difference between solubility parameters of water and PLGA as well as water and drugs, indicates that PLGA and ARVs are not miscible with water that contradicts hydrophilic properties of TFV. Based on this, the Hildebrand solubility parameter is not an accurate measurement of drug-carrier compatibility for our system. This might be attributed to the fact that the Hildebrand solubility parameter is often used 
to predict nonpolar and slightly polar systems and does not include hydrogen bonding ${ }^{322}$. Thus, RDF plots were created to predict the hydrogen bonding interactions between each drug and PLGA.

Table 3.3. Cohesive energy densities obtained from MD simulations and calculated values. a. CED calculated using MD simulations and b. CED calculated using experimental values in literature or calculated based on group contributions.

\begin{tabular}{|c|c|c|c|c|}
\hline Compound & $\begin{array}{c}\text { MD simulated Cohesive } \\
\text { Energy Density (MPa) }\end{array}$ & $\begin{array}{c}\text { MD Simulated Solubility } \\
\text { Parameter }(\mathbf{M P a})^{\mathbf{1 / 2}}\end{array}$ & $\begin{array}{c}\text { Solubility Parameter in } \\
\text { Literature (MPa) }\end{array}$ & \% difference \\
\hline water & 2013 & 44.866 & 47.9 & $-7 \%$ \\
\hline PLGA & 638 & 25.259 & 23.1 & $9 \%$ \\
\hline TFV & 689 & 26.249 & 23.35 & $11 \%$ \\
\hline RAL & 601 & 24.515 & 22.76 & $7 \%$ \\
\hline DAP & 514 & 22.672 & 19.94 & $12 \%$ \\
\hline
\end{tabular}

Radial Distribution Function. The first peak in RDF plot with $g_{\mathrm{ab}}(r)>1$ indicates interactions between the $a$ and $b$ atoms. Figure $\mathbf{3 . 3}$ identified forcefield types for atoms in each drug, water, and PLGA that showed distinguishable peaks in the RDF plot. As shown in Figure 3.4, no peak was evident in the RDF plots between oxygen atoms in ether (02s) or carbonyl (01=) groups of PLGA and hydrogen atoms of water (h10). In addition, no peak was observed in the RDF plot between the hydrogen atom in PLGA ( $\mathrm{h} 1)$ and the oxygen atom in water $\left(\mathrm{o} 2^{*}\right)$. This indicates no apparent interactions between hydrogen bonding moieties probably because hydrogen connected to carbon in PLGA is not electropositive enough to form a hydrogen bond with oxygen in water and this observation is consistent with the hydrophobicity of the polymer. 

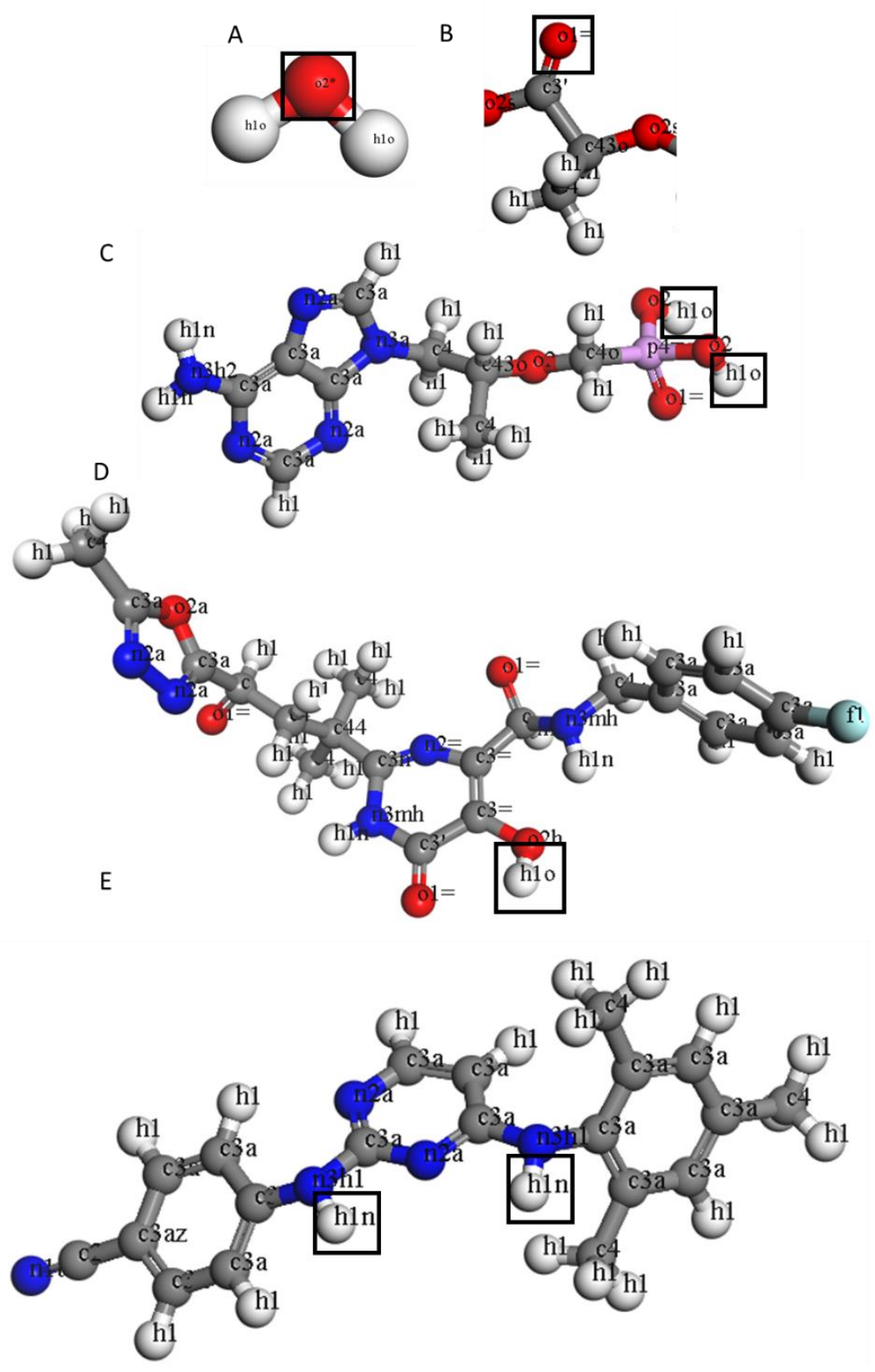

Figure 3.3. Identification of atoms participating in intermolecular interactions between drugs and water/polymer. A) oxygen atom (02*) in water; B) oxygen atom (o1=) in PLGA; C) hydrogen atom (h10) in TFV; D) hydrogen atom (h10) in RAL; 
and E) hydrogen atom (h1n) in DAP. Forcefield types are showed for all atoms and interacting atoms are identified by boxes.
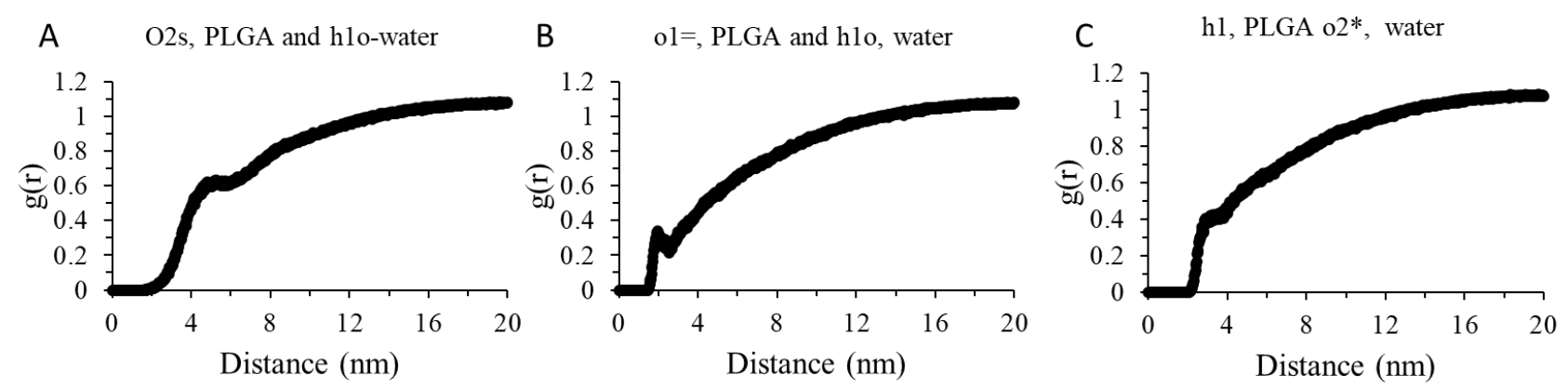

Figure 3.4. RDF for intermolecular interactions between A) oxygen atom (o2s) in the ether group of PLGA and hydrogen atom (h10) in water; B) oxygen (01=) in the carbonyl group of PLGA and oxygen (o2*) in water; and C) hydrogen (h1) in PLGA and oxygen in water.

The RDF plots for functional atoms in combinations of TFV and either water, PLGA, or a mixture of water and PLGA are shown in Figure 3.5. Only the RDF plots having distinct peaks for hydrogen and oxygen pairs are shown in this figure. The sharp peak at $1.8 \AA$ between the hydrogen atom connected to the oxygen atom in TFV (h10) and the oxygen atom in water suggests that there is hydrogen bonding between both molecules. Similar interactions were observed between hydrogen connected to oxygen in TFV (h10) and oxygen in the carbonyl group of PLGA (01=). Interestingly, hydrogen bonding was not observed between TFV and PLGA in the presence of water, but it was still present between TFV and water in the presence of the polymer. This suggests that hydrogen bonding between water and drug is stronger than between polymer and drug. 
TFV + PLGA or
$\mathrm{TFV}+$ Water

A

hlo, TFV and ol=, PLGA

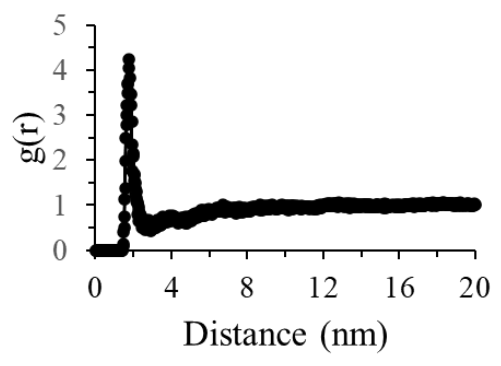

C

hlo, TFV and ol=, PLGA
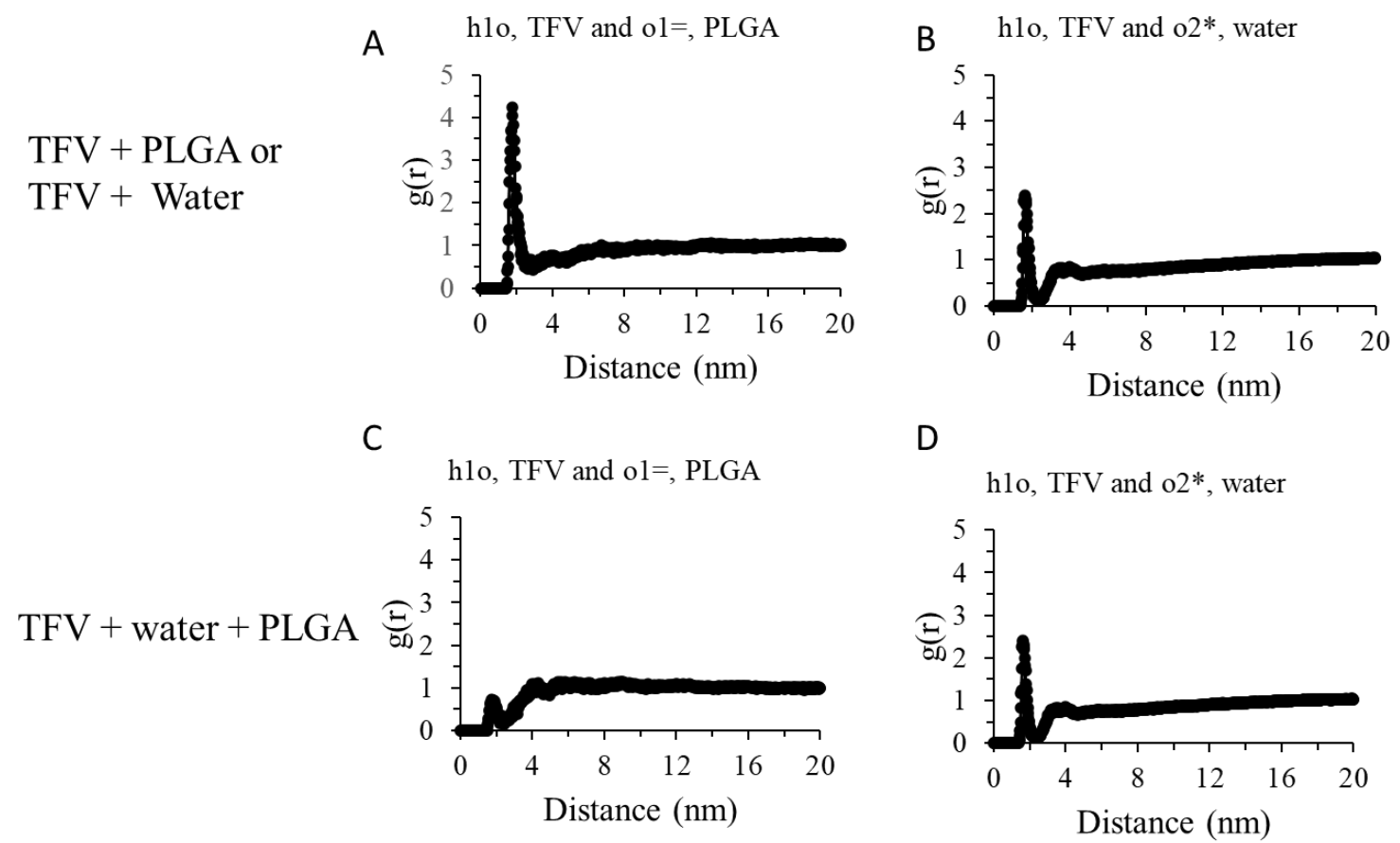

D

hlo, TFV and o2*, water

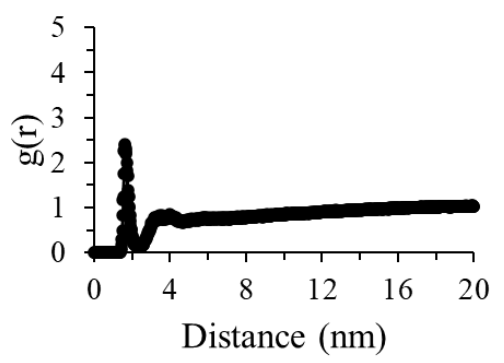

Figure 3.5. RDF for intermolecular interactions between hydrogen atom (h10) in TFV and oxygen atoms ( $01=$ or $\left.02^{*}\right)$ in A) PLGA in the absence of water; B) water in the absence of PLGA; C) PLGA in the presence of water and D) water in the presence of PLGA.

For RAL, hydrogen bonding between the hydrogen atom connected to the oxygen atom in RAL (h10) and oxygen atoms of water $\left(02^{*}\right)$ was observed at the distance of $1.8 \AA$ but the peak in RDF was less sharp than observed for TFV, which is in agreement with the lower solubility of RAL in water (Figure 3.6). However, there was evidence of strong hydrogen bonding between the hydrogen atoms connected to the oxygen atom in $\mathrm{RAL}(\mathrm{h} 10)$ and the oxygen atom of the carbonyl group in PLGA (01=). The interactions between RAL and PLGA are as strong as between RAL and water molecules when all three components are present in the 
system.
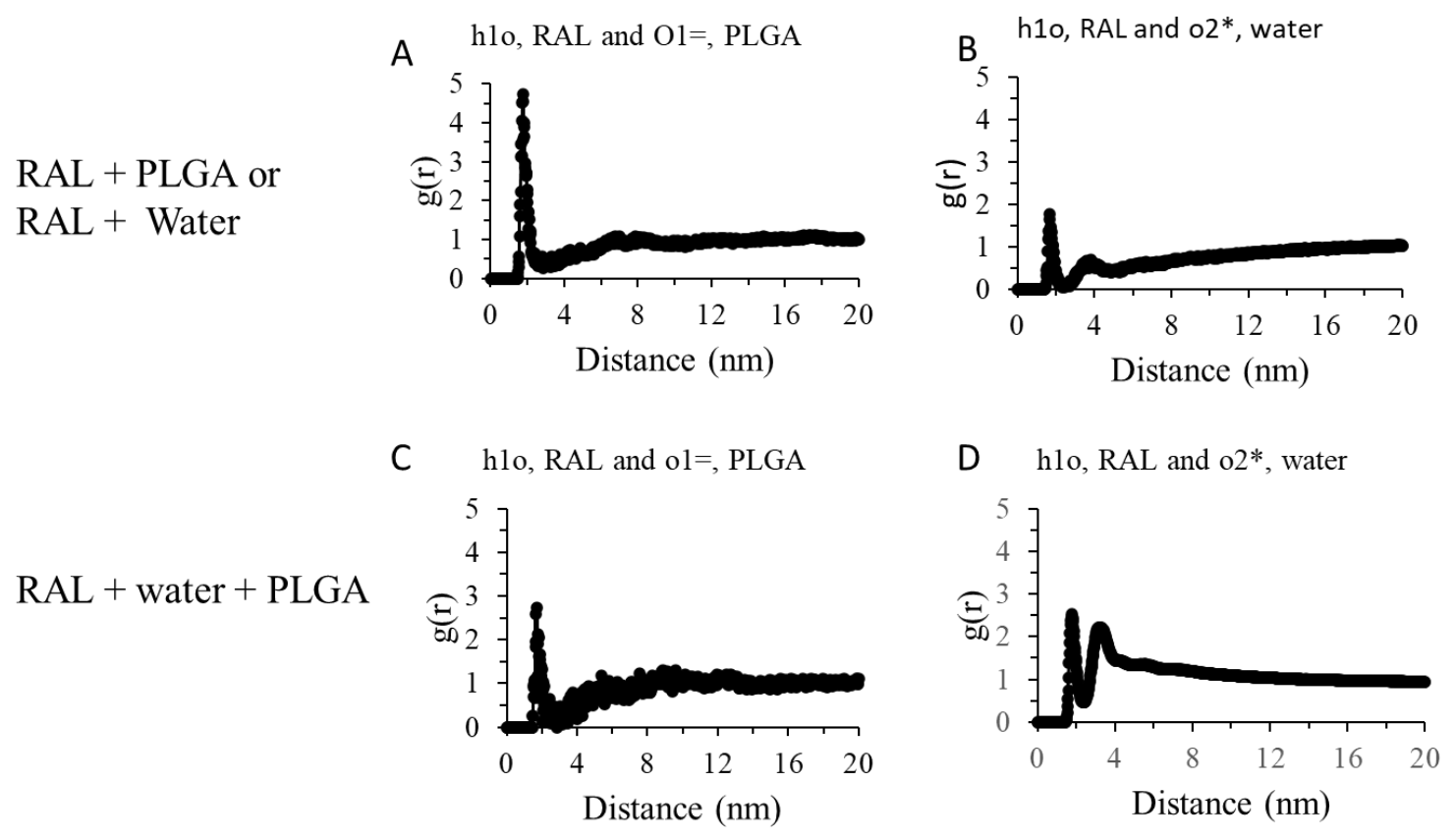

Figure 3.6. RDF for intermolecular interactions between hydrogen atom (h10) in RAL and A) oxygen atom ( $01=)$ in PLGA in the absence of water; B) oxygen atom $\left(02^{*}\right)$ in water in the absence of PLGA; C) oxygen atom (01=) in PLGA in the presence of water; and D) oxygen atom (02*) in water in the presence of PLGA.

No interactions between DAP and water molecules were detected due to the hydrophobicity of this drug. Hydrogen bonds formed between the hydrogen atom connected to the nitrogen atom in DAP $(\mathrm{h} 1 \mathrm{n})$ and the oxygen atom of the carbonyl group in PLGA (01=) and hydrogen bonding was still detected in the presence of water, even though it was much weaker (Figure 3.7). No difference in 
interactions between DAP and water was observed in the absence or presence of PLGA.

$\mathrm{DAP}+\mathrm{PLGA}$ or

$\mathrm{DAP}+$ Water

A hln, DAP and ol=, PLGA

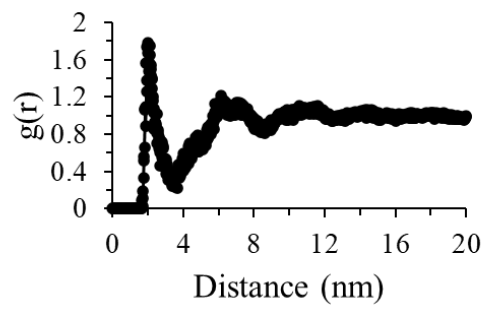

C

$\mathrm{DAP}+$ water + PLGA

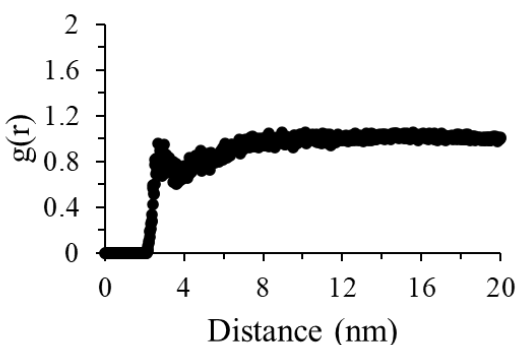

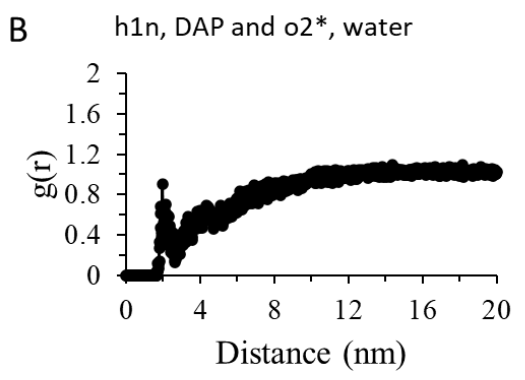

D hln, DAP and o2*, water

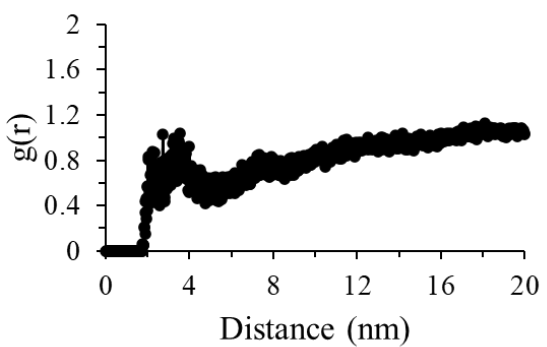

Figure 3.7. RDF for intermolecular interactions between hydrogen atom (h1n) in DAP and oxygen atom ( $01=)$ in A) PLGA in the absence of water; B) water in the absence of PLGA; C) PLGA in the presence of water; and D) water in the presence of PLGA.

Hydrogen Bonding. To further evaluate the hydrogen bonding strength for different drugs and the polymer, we compared the charges on individual hydrogen and oxygen atoms. In TFV, the charge of the hydrogen atom connected to oxygen (h10) and the hydrogen atom connected to nitrogen atoms (h1n) were 0.41 and 0.35 , respectively, showing that the hydrogen atom connected to the oxygen atom is more electropositive. Moreover, the oxygen in the carbonyl group $(\mathrm{C}=\mathrm{O})$ and the oxygen in the ether group (C-O-C) of PLGA were -0.45 and -0.27 , respectively. Therefore, the double-bonded oxygen atom $(\mathrm{C}=\mathrm{O})$ has a higher tendency to form 
hydrogen bonding compare with single-bonded oxygen atom (C-O-C). However, the electrostatic interactions between opposite charges include both columbic interactions and hydrogen bonds formed between the drug and water or the drug and polymer. To study the hydrogen bonding contribution, the distance between the hydrogen atom and the acceptor atom was measured (Figure 3.8). Among all different atoms in each drug, the distance between the hydrogen atom connected to oxygen in TFV (h10), the hydrogen atom connected to oxygen in RAL (h10), and the hydrogen atom in DAP (h1) and the oxygen atom in the carbonyl group of PLGA (01=) was $\leq 2.8 \mathrm{~nm}$, indicating moderately strong hydrogen bonding.

Based on the experimental values for loading of different ARVs, RAL NPs possessed the highest loading and TFV NPs had the lowest loading. In addition, the predictions from MD simulations verified the fact that encapsulation capability of PLGA NPs is higher for RAL, relative to TFV due to stronger hydrogen bonding between RAL and carboxyl group of PLGA. One possible reason for weaker hydrogen bonding between TFV and PLGA might be the attributed to the steric hindrance in phosphoric group of TFV may decrease the strength of the hydrogen bonds. 


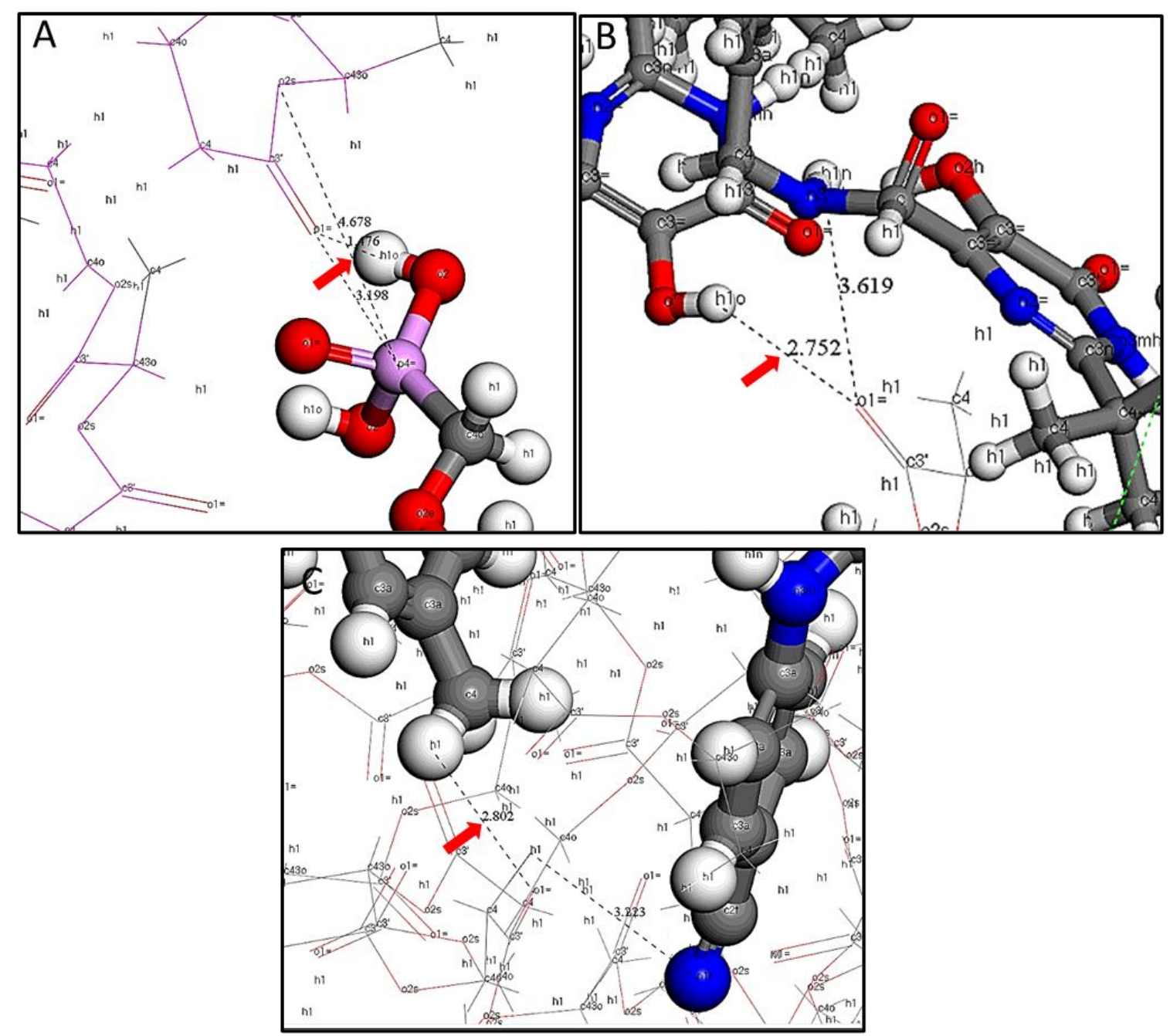

Figure 3.8. Visualization of hydrogen bond formation between the oxygen atom (o1=) of PLGA and A) hydrogen atom (h10) of TFV, B) hydrogen atom (h10) of RAL, and C) hydrogen atom (h1) of DAP. Hydrogen bonds are shown with red arrows. 


\section{Conclusions}

One of the challenges in delivering small hydrophilic therapeutics encapsulated in polymeric NP is low encapsulation efficacy. In this study, we studied the interactions between three different ARVs and PLGA and their effect on the encapsulation efficacy of PLGA NPs. Our experimental data has shown that the encapsulation efficacy of TFV was as low as 15\%; however, the loading for RAL and DAP NPs was much higher (> 70\%). First, the solubility parameter of each model compound was computed and compared to literature values to validate and verify the simulation model. MD simulations indicated that hydrogen bonding between TFV and PLGA in the presence of water is weaker than between TFV and water in the same system, causing the drug to leach into the aqueous phase in the process of NP fabrication and causing low encapsulation efficacy. On the other hand, hydrogen bonding between the oxygen atom in the carbonyl group of PLGA and the hydrogen atom connected to the oxygen atom in RAL was stronger than RAL and the oxygen atom in water, resulted in high encapsulation efficacy for RAL NPs.

This study demonstrated the potential of MD simulations to accurately predict the encapsulation capability of polymeric NPs by evaluating the intermolecular interactions between the drug and polymer. This approach has the potential to be used to optimize formulation of polymeric NPs as well as tailor-make the polymer nanoparticles to achieve higher encapsulation efficacy, especially for hydrophilic small drugs. Moreover, this computational method can be applied to other polymeric delivery systems to evaluate the compatibility of drug and dosage 
form and predict encapsulation efficacy of polymeric drug carriers. In the future, the effect of adding different functional groups on polymer chain can be evaluated to tailor the interactions between TFV and PLGA to improve the loading of NPs. 


\section{CHAPTER 4}

\section{PROBIOTIC FIBERS AS AN ALTERNATIVE DOSAGE FORM FOR BACTERIAL VAGINOSIS TREATMENT}

\section{Introduction}

Bacterial vaginosis $(\mathrm{BV})$ is the most common vaginal infection in women between the ages of 15 to 44 , impacting $~ 30 \%$ of women in the U.S. and globally. Within the healthy female reproductive tract (FRT), microorganisms belonging to the genus Lactobacillus are the most abundant, and aid in maintaining host homeostasis and providing innate defense against incoming pathogens ${ }^{5}$. However, for women afflicted with BV, the microbial ecology of the reproductive tract is altered, and resident lactobacilli are replaced by an overgrowth of pathogenic vaginal anaerobes such as Gardnerella vaginalis (G. vaginalis), as well as Atopobium vaginae, Prevotella, Peptostreptococcus, and Bacteroides spp. This increased diversity and dysbiosis of the vaginal microbiota is associated with an elevated risk of adverse pregnancy outcomes, post-surgical infections, acquisition of sexually transmitted infections (STIs), and other deleterious health outcomes ${ }^{5}$.

Several antibiotics have been used to manage BV, including metronidazole, clindamycin, and tinidazole, which are administered orally or topically in the form of tablets, creams, and gels ${ }^{323}$. Currently recommended BV treatments consist of 
oral metronidazole tablets, taken twice daily for one week; metronidazole gel applied once daily for 5 days; or clindamycin cream applied once a day for one week $^{5}$. While $\sim 80 \%$ of infections are initially effective with these approaches, chronic antibiotic usage has been shown to contribute to antibiotic-resistance, while also promoting adverse gastrointestinal effects and opportunistic yeast infections $^{323}$. More importantly, there is no cure for BV, and nearly $50 \%$ of women experience recurrence of infection within 12 months post-treatment ${ }^{323}$.

Relative to antibiotic-based strategies, the administration of probiotics, or beneficial bacterial species, has been shown to provide anti-inflammatory effects and help in modulating host immunity. The administration of probiotics via orallyor topically-administered dosage forms may help to disrupt abnormal microflora and restore balance to the vaginal microbiota ${ }^{324}$. Thus, new approaches have begun to focus on the delivery of probiotics to stabilize the vaginal microbiome and prevent the growth of BV-associated bacteria. Of the probiotic candidates, lactobacilli are the most abundant, and help to promote vaginal health by producing a variety of molecules including: lactic acid to decrease the vaginal $\mathrm{pH}$; biosurfactant-containing collagen-binding proteins to compete against pathogenic species for vaginal epithelial adherence; and hydrogen peroxide and bacteriocins, which act as antimicrobials and enhance host immune responses ${ }^{325}$. In fact, mechanisms such as auto-aggregation of Lactobacillus, Lactobacillus adherence to epithelial cells, or the co-aggregation of Lactobacillus and pathogenic microorganisms have been shown to modulate the composition of the healthy vaginal microbiota and to improve endogenous microflora ${ }^{326-328}$. 
Given their role in mediating pathogenesis, several studies have investigated the efficaciousness of different Lactobacillus species in inhibiting BV infection ${ }^{326}$. Previous studies have shown that Lactobacillus acidophilus ( $L$. acidophilus) is one of the few taxa with promising evidence of microbiological and clinical cure ${ }^{329,330}$, and are believed to impact BV infection by producing hydrogen peroxide and bacteriocins that have a wide range of antibacterial activities ${ }^{323}$. One study showed that L. acidophilus GLa-14 significantly reduces G. vaginalis adherence to HeLa cells in vitro by regulating immune responses such as macrophage activation, thereby indicating or promoting anti-BV effect ${ }^{331}$. In another study, two Lactobacillus species, L. fermentum MG901 and L. plantarum MG989, were individually co-cultured with $G$. vaginalis and both strains significantly decreased $G$. vaginalis viability ${ }^{332}$. Furthermore, studies in a murine model have shown that combining the oral delivery of capsules containing either L. acidophilus GLa-14, L. rhamnosus HN001, or both probiotics with lactoferrin $\mathrm{RCX}^{\mathrm{TM}}$ reduced $G$. vaginalis vaginal colonization and inhibited $G$. vaginalisinduced expression of cytokines involved in adaptive immunity, and that of these formulations, L. acidophilus GLa-14 was the most effective ${ }^{331}$.

Lasty, clinical trials have demonstrated promising outcomes with the administration of lactobacilli to treat BV. A clinical study with women infected with $\mathrm{BV}$, candida vaginitis, or both showed that daily ingestion of yogurt containing $10^{8}$ colony forming units (CFU) L. acidophilus $/ \mathrm{mL}$ significantly increased the number of $L$. acidophilus-positive vaginal cultures (86\%), relative to those who ingested pasteurized yogurt with no probiotic (36\%), indicating the potential of $L$. acidophilus 
in treating $\mathrm{BV}^{329}$. Another clinical study evaluated the oral co-administration of two Lactobacillus species, L. rhamnosus $G R-1$ and $L$. reuteri $R C-14\left(10^{9} \mathrm{CFU}\right.$ each per capsule), for thirty days after a conventional twice-daily oral metronidazole tablet regimen spanning one week, and demonstrated a $48 \%$ decrease in $\mathrm{BV}$ infection after $28 \mathrm{~d}$, relative to antibiotic-only treatment ${ }^{333}$. Similarly, a clinical trial with women suffering from BV recurrence demonstrated that the oral administration of one capsule per day for 7 days, that contained $8 \times 10^{9} \mathrm{CFU}$ of $L$. acidophilus, $L$. rhamnosus and Streptococcus thermophilus in total per capsule, resulted in recurrence rates as low as $15 \%$, relative to the placebo group (45\%) ${ }^{334}$.

Despite these advances, some of the challenges confronting oral probiotic delivery include transport and degradation associated with exposure to the acidic environment of the gastrointestinal tract, often necessitating increased dosing and administration frequency to achieve colonization and subsequent effect ${ }^{335}$. Localized intravaginal probiotic administration may circumvent these challenges and offer a promising alternative to oral administration strategies. Vaginal dosage forms, including gel, cream, and capsule formulations have been used to locally deliver probiotics to the FRT, and to induce local acidification and epithelial colonization, thereby preventing the adherence of pathogenic bacteria ${ }^{334,336,337}$. In clinical trials, 95 patients who were administered vaginal capsules containing a mixture of L. gasseri, L. casei., L. rhamnosus, L. fermentum, and P. acidilactici for 5 days experienced reduced vaginal discharge and a higher clinical cure rate relative to placebo capsules after two menstrual cycles ${ }^{338}$. In another clinical trial, vaginal tablets containing $\geq 10^{7} \mathrm{CFU}$ L. acidophilus per tablet reduced BV infection 
by up to $76 \%$, after a two week, 1-2 tablet(s) per day regimen ${ }^{339}$. Yet, similar to oral dosage forms, most vaginal dosage forms require frequent daily or twice-daily administration over a one to two-week duration. These regimens can be inconvenient and may also induce discomfort, due to product leakage. The most effective dosage form to date, which reduced BV infection by up to $88 \%$, required the administration of 1 to 2 vaginal tablet(s) containing $\geq 10^{7} \mathrm{CFU} L$. acidophilus/tablet and $0.03 \mathrm{mg}$ estriol for 6 days ${ }^{339}$.

To avoid the challenges and inconvenience of daily treatment regimens, a delivery vehicle that offers prolonged delivery ( $>1 \mathrm{wk}$ ) may be desirable; however, few dosage forms are currently available to achieve sustained-release of probiotics. To date, only one dosage form, in the form of a pod-based intravaginal ring, has shown the potential to provide sustained-release of probiotics for intravaginal application ${ }^{340}$. However, rings may be susceptible to biofilm formation and yeast adhesion, posing an additional challenge to BV treatment ${ }^{5}$. Recently, electrospun fibers have emerged as a viable platform to deliver prophylactic and therapeutic agents ${ }^{341}$ and probiotics ${ }^{342,343}$ for viral and bacterial female reproductive health applications. Hydrophilic polymers such as polyethylene oxide (PEO), polyvinyl alcohol (PVA) and polyvinyl pyrrolidone (PVP) have been used to fabricate fibers that incorporate different bacteria including Pseudomonas, Zymomonas, Escherichia, and Lactobacillus for a variety of applications ranging from synthetic biofilm-based membrane bioreactors to the development of stable solid probiotic dosage forms such as electrospun nanofibrous matrices ${ }^{342,344,345}$. Polymeric fibers made from PEO and PVA offer rapid dissolution and a variety of 
attributes including bioadhesivity, which may increase retention for intravaginal applications, and as a result may extend probiotic (or other active agent) residence time, thereby decreasing the risk of recurrence ${ }^{323,341}$. In addition to these attributes, encapsulant release by electrospun fibers can be tuned by changing the material, electrospinning parameters ${ }^{346}$, and architecture $^{341}$ which may enable the prolonged delivery of lower active agent doses within the FRT. Lastly, fibers have been shown to enhance encapsulant stability and half-life ${ }^{347}$, which is particularly important for the incorporation of live cells.

Previous work in our group has focused on the development of electrospun fibers that deliver proteins, and anti(retro)virals against viral ${ }^{125,128,273,348-350}$ and bacterial infections in the FRT and oral cavity ${ }^{351}$, respectively. In particular, our recent studies in an HSV-2 murine model demonstrated the efficacy and preliminary safety of intravaginally administered, single and multilayered PEO fibers $^{273,349}$. Here we sought to extend this work to develop rapid-dissolve fibers that incorporate $L$. acidophilus, as a first step in developing a sustained-release probiotic fiber platform. We first assessed the ability of PEO fibers to function as an inert and safe delivery platform in vivo, using a traditional antibiotic treatment as early proof-of-concept in a murine model of G. vaginalis infection. Based on these promising results with a traditional treatment strategy, we subsequently formulated fiber scaffolds made of two different water-soluble polymers, PEO and PVA, to provide high loading, viability, stability, and release of $L$. acidophilus in vitro. The optimal PEO and PVA formulations were then evaluated for their ability to inhibit $G$. vaginalis viability in broth co-culture and epithelial-G. vaginalis co- 
colonization assays. These studies suggest that PEO fibers are preliminarily safe and capable of intravaginally delivering effective therapeutics in a murine model of G. vaginalis infection, and further highlight the versatility of electrospun fibers to functionally incorporate probiotics against in vitro infection.

\section{Materials and Methods}

\section{Fabrication and characterization of metronidazole (met)-containing PEO fibers}

Blank and metronidazole (met)-containing polyethylene oxide (PEO, 600 $\mathrm{kDa}$ ) fibers were electrospun to evaluate the preliminary safety and efficacy of fibers in a murine model of $G$. vaginalis infection. To prepare the polymer solution for electrospinning, a $5 \% \mathrm{w} / \mathrm{w}$ PEO solution was formulated in a final electrospinning volume of $3 \mathrm{~mL}$ dimethylformamide and was incubated for $30 \mathrm{~min}$ at room temperature. The mentioned polymer percentage (5\% PEO) was based on a previously optimized formulation that resulted in bead-free and well-defined fiber structure ${ }^{273,349}$. Then, $100 \mu \mathrm{g}$ met per mg polymer, was added to the polymer solution, vortexed thoroughly, and incubated for $30 \mathrm{~min}$. The resulting metcontaining or blank PEO solution was electrospun using a flow rate of $0.3 \mathrm{~mL} / \mathrm{hr}$, with an applied voltage of $20 \mathrm{kV}$. The resulting fiber mats were collected on a rotating, $8 \mathrm{~mm}$ outer diameter stainless steel mandrel, positioned $15 \mathrm{~cm}$ from the blunt needle tip of the syringe. The sample flow rate was regulated by an infusion pump (Fisher Scientific, Pittsburgh, PA) and the voltage was applied using a high 
voltage power supply (Spellman CZE 1000R). After electrospinning, the fiber was removed from the mandrel and desiccated overnight at room temperature.

To measure the loading of met-containing fibers, $3 \mathrm{mg}$ of fiber were dissolved in $1 \mathrm{~mL} 1 \mathrm{X}$ phosphate buffered saline (PBS). This solution was diluted by adding $100 \mu \mathrm{L}$ of sample to $900 \mu \mathrm{L}$ PBS (1:10) and the amount of met was quantitated via reverse phase high performance liquid chromatography with UV detection (HPLC-UV), as previously described ${ }^{352}$. Briefly, diluted samples were separated on a Sunfire $4.6 \times 250 \mathrm{~mm} \mathrm{C18}$ column (Waters) using a mobile phase

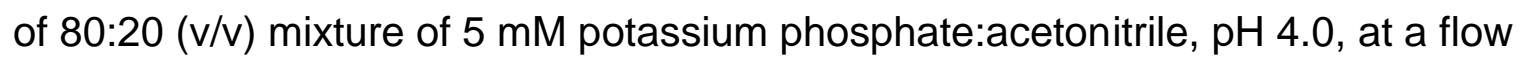
rate of $1 \mathrm{~mL} /$ minute. Eluates were monitored at $324 \mathrm{~nm}$ (Waters 2487 detector) and quantified relative to an external standard of purified met.

\section{Preliminary preclinical assessment of PEO fibers to safely deliver vaginal therapies}

As a first test of the feasibility of the in vivo fiber approach, the safety of fibers and the fiber placement approach was evaluated in a murine model of $G$. vaginalis infection. Ten female 6-8-week-old C57BI/6J mice were purchased from Jackson Laboratories in June 2018 and allowed to convalesce in the Washington University facility for 1 week prior to experimentation. All mice were first given an intraperitoneal injection of $\beta$-estradiol-17-valerate $(0.5 \mathrm{mg}$ in $100 \mu \mathrm{L}$ sterile sesame oil) to synchronize the estrus cycle; this is standard practice in vaginal bacterial infection models. Three days later, mice were given a second $\beta$-estradiol injection and vaginal washes were collected by inserting a sterile P20 pipette with $60 \mu \mathrm{L}$ 
PBS and pipetting up and down. Then microdissection forceps were used to carefully insert $6 \mathrm{~mm}$ PEO discs into the vaginas of 5 mice, while 5 mice were untreated (negative controls). Immediately following fiber insertion, all 10 of these mice were then intravaginally infected with $\sim 10^{8} \mathrm{CFU}$ of a streptomycin-resistant clinical isolate of G. vaginalis (JCP8151B, GenBank JX860320) in $20 \mu \mathrm{L}$ PBS using a P20 pipette. The next day, vaginal washes were collected and plated for CFU and vaginas were collected and fixed in $4 \%$ paraformaldehyde for histological analysis.

To begin to assess the potential therapeutic benefit of fiber delivery with a known standard therapy, twenty female C57BI/6J mice were estrogenized as above. The mice were divided into two (10 mice each) experimental arms: "prevention" and "treatment." For the "prevention" experiment, microdissection forceps were used to carefully insert $6 \mathrm{~mm}$ met-PEO discs into the vagina of 5 mice, while 5 mice received blank fibers (negative control). Immediately following fiber insertion, all 10 of these mice were then intravaginally infected with $\sim 10^{8} \mathrm{CFU}$ G. vaginalis in $20 \mu \mathrm{L}$ PBS using a P20 pipette. The next day, after $\sim 24 \mathrm{hr}$, vaginal washes were collected and then the mice were sacrificed to collect vaginal and uterine horn tissue, which were homogenized and plated for CFUs by serial dilution and plating on selective media (NYCIII + strep). Vaginal washes were likewise plated for CFU counts.

For the "treatment" arm of the experiment, 10 mice were inoculated with $G$. vaginalis as described above. The next day, $\sim 24 \mathrm{hr}$ later, vaginal washes were collected from all mice to determine the level of $G$. vaginalis infection prior to 
treatment by CFU plating. Immediately after vaginal wash collection, 5 mice were given met-PEO fibers and the remaining 5 were given blank fibers. The following day (thus $48 \mathrm{hr}$ after $\mathrm{G}$. vaginalis inoculation, $24 \mathrm{hr}$ after fiber administration), vaginal washes and vaginal and uterine tissue were collected, serially diluted, and plated as above. G. vaginalis CFUs were counted from the vaginal wash and vaginal/uterine tissue homogenate dilutions and H\&E-stained sections were examined.

\section{Histological analysis}

Following fixation, vaginas were processed for histological analysis by embedding in paraffin, sectioning, and staining with hematoxylin and eosin by the Department of Developmental Biology Histological Core at Washington University School of Medicine. Stained sections were visualized on an Olympus BX61 microscope.

\section{Bacterial cultures}

L. acidophilus was purchased from American Type Culture Collection (ATCC 4356) and a streptomycin-resistant clinical isolate of $G$. vaginalis (JCP8151B, GenBank JX860320) was generously provided by Dr. Amanda Lewis (Washington University, St. Louis, MO). L. acidophilus was grown using de Man, Rogosa, and Sharpe (MRS, Sigma 69966) broth and agar plates, both supplemented with $0.1 \%$ Tween 80 . G. vaginalis was grown using New York City III (NYC III) broth and agar plates, both supplemented with 10\% heat-inactivated horse serum (Gibco 26050088). The NYC III agar plates contained $1 \mathrm{mg} / \mathrm{mL}$ 
streptomycin sulfate salt (Sigma S9137) to select for the antibiotic-resistant $G$. vaginalis strain.

Bacterial cultures were initially prepared from frozen stocks using an inoculation loop to streak frozen culture on the appropriate agar plates. Both $L$. acidophilus and $G$. vaginalis were initially cultured on agar plates under anaerobic conditions and maintained at $37^{\circ} \mathrm{C}$ in $5 \%$ hydrogen, $5 \%$ carbon dioxide, and balanced with nitrogen using a Bactronez Anaerobic Workstation (Shel Lab). The incubation time to observe the growth of L. acidophilus on MRS plates was $48 \mathrm{hr}$, while G. vaginalis cultures were incubated for $72 \mathrm{hr}$ on NYC III agar plates due to slower colony formation. For all bacterial sub-cultures, a single bacteria colony was selected from the agar plate and cultured in $1 \mathrm{~mL}$ of MRS or NYC III broth in a closed microcentrifuge tube for 48 or $72 \mathrm{hr}$ at $37^{\circ} \mathrm{C}$, to minimize exposure to air. The bacteria were subsequently sub-cultured in broth by diluting $200 \mu \mathrm{L}$ of $L$. acidophilus with $9.8 \mathrm{~mL}$ of MRS broth (1:50 dilution) and $20 \mu \mathrm{L}$ of $\mathrm{G}$. vaginalis with $980 \mu \mathrm{L}$ NYC III broth (1:50 dilution). Growth curves were evaluated prior to conducting experiments, to determine the exponential phase of bacterial growth, and to compare the $\mathrm{OD}_{600}$ absorbance values with respect to time. To generate the growth curves, each bacterium was sub-cultured by adding $0.1 \mathrm{~mL}$ of the first L. acidophilus or G. vaginalis sub-culture solution to $10 \mathrm{~mL}$ media and grown until the stationary phase was achieved. The correlation between the $\mathrm{OD}_{600}$ and respective CFU counts was determined by plating serial dilutions of each culture on MRS and NYC III agar plates for L. acidophilus and G. vaginalis, respectively. 
One $\mathrm{OD}_{600}$ of $L$. acidophilus and $G$. vaginalis was found to be equivalent to $5.8 \mathrm{x}$ $10^{7}$ and $1.3 \times 10^{9} \mathrm{CFU} / \mathrm{mL}$, respectively.

During the late exponential phase $\left(\mathrm{OD}_{600}\right.$ between 2 and 7), L. acidophilus was harvested for fiber fabrication. L. acidophilus cultures in MRS growth media were centrifuged at $3000 \times \mathrm{g}$ for $10 \mathrm{~min}$ at room temperature $\left(25^{\circ} \mathrm{C}\right)$. The bacterial pellet was then either resuspended in MilliQ water, MRS broth, or MRS broth with $5 \% \mathrm{v} / \mathrm{v}$ glycerol in preparation for electrospinning. To maximize the loading of $L$. acidophilus into fibers and to assess the proper media for co-culture assays, $L$. acidophilus and $G$. vaginalis growth rates were evaluated in both MRS and NYC III broths and the concentration of each bacterial species was determined by measuring the $\mathrm{OD}_{600}$ at various time points over $48 \mathrm{hr}$ or $72 \mathrm{hr}$, respectively.

\section{Bacterial lyophilization}

For the lyophilized $L$. acidophilus electrospun group, MRS media containing $6 \%(\mathrm{w} / \mathrm{v})$ skim milk and $4 \%(\mathrm{w} / \mathrm{v})$ trehalose was added to a fresh $L$. acidophilus pellet from a first sub-culture. The suspension was then aliquoted to $5 \mathrm{~mL}$ solutions in cryovials and frozen at $-80^{\circ} \mathrm{C}$ for a minimum of $2 \mathrm{hr}$. The frozen $L$. acidophilus suspensions were then lyophilized for $48 \mathrm{hr}$, resulting in a dry powder that was then stored at $-80^{\circ} \mathrm{C}$. The viability of post-lyophilized L. acidophilus was determined by CFU count.

\section{Fabrication of $L$. acidophilus-containing PEO and PVA fibers}

Polyethylene oxide (PEO, $600 \mathrm{kDa}$ ) and PVA (146-186 kDa, 87\% hydrolyzed) were prepared to fabricate fibers using a uniaxial electrospinning 
approach. Different formulations of 5\% w/w PEO and 10\% w/w PVA fibers were electrospun in either water, MRS broth, or MRS broth with $5 \%$ glycerol, with or without the addition of fresh or lyophilized L. acidophilus, to determine the most favorable electrospinning conditions to maximize L. acidophilus viability. The mentioned polymer percentages (5\% PEO, 10\% PVA) were previously optimized concentrations that resulted in bead-free and well-defined fiber structures 273,349 . Formulations, incorporating lyophilized $L$. acidophilus, were evaluated to assess the impact of lyophilization on bacterial cell viability. Different concentrations of $L$. acidophilus in various solutions (detailed below) were added to the PEO or PVA solutions to determine the maximum concentration of viable probiotics that could be incorporated into fibers.

Each probiotic fiber formulation was optimized by adjusting the electrospinning flow rate, applied voltage, and the distance between the syringe needle and mandrel. To fabricate the fibers, PEO and PVA solutions were prepared at $5 \%$ and $10 \% \mathrm{w} / \mathrm{v}$ respectively, in a final electrospinning solution volume of $3 \mathrm{~mL}$. The day before electrospinning, PEO or PVA was added to either $2 \mathrm{~mL}$ of Milli-Q water, MRS broth, or MRS with glycerol, and incubated overnight at $37^{\circ} \mathrm{C}$. The next day, fresh $L$. acidophilus was centrifuged $(3000 \mathrm{x} \mathrm{g})$ in a $50 \mathrm{~mL}$ centrifuge tube and an aliquot of the pellet, containing $5 \times 10^{6}, 5 \times 10^{7}$, or $5 \times 10^{8}$ CFU L. acidophilus/mg polymer, was resuspended in $1 \mathrm{~mL}$ MRS broth and vortexed thoroughly. For lyophilized $L$. acidophilus, $1 \mathrm{mg}$ of dry bacteria was weighed and suspended in $1 \mathrm{~mL}$ MRS broth, plated to assess CFU count per mg, and the desired amount of bacteria was added to the polymer solution. After 
incubating bacteria with the polymer solution for $30 \mathrm{~min}$ at $37^{\circ} \mathrm{C}$, PEO or PVA solution was electrospun using a flow rate of $0.3 \mathrm{~mL} / \mathrm{hr}$ (for water) or $0.25 \mathrm{~mL} / \mathrm{hr}$ (for MRS and MRS with glycerol).

The required voltage was either $20 \mathrm{kV}$ (for water) or $25 \mathrm{kV}$ (for MRS or MRS with glycerol). The resulting fiber mats were collected on a rotating, $8 \mathrm{~mm}$ outer diameter stainless steel mandrel, positioned $15 \mathrm{~cm}$ from the blunt needle tip of the syringe. After electrospinning, the fiber was removed from the mandrel and desiccated overnight at room temperature. Each fiber formulation was tested for bacterial viability as a function of time in $-20^{\circ} \mathrm{C}, 4^{\circ} \mathrm{C}$, and $25^{\circ} \mathrm{C}$ storage conditions for up to 3 months.

\section{Probiotic viability in PEO and PVA fibers}

The CFU counts of $L$. acidophilus in the initial electrospinning solution were compared to the CFU counts in fibers immediately after electrospinning to determine changes in L. acidophilus viability as a result of the electrospinning process. To determine CFU counts, a series of 10 -fold dilutions were made of the initial electrospinning solution and of fibers dissolved in MRS broth. Each dilution was plated on an MRS agar plate, incubated in anaerobic conditions at $37^{\circ} \mathrm{C}$, and evaluated for CFU counts after $48 \mathrm{hr}$.

\section{Probiotic fiber morphology}

The morphology of PEO and PVA electrospun fibers (with or without $L$. acidophilus) was characterized using scanning electron microscopy (SEM). Fibers were placed on carbon tape, sputter-coated with a layer of palladium/gold alloy, 
and imaged using Supra 35 SEM (Zeiss, Oberkochen, Germany). Images were analyzed using ImageJ software and the average fiber diameter of each formulation was determined by drawing a line across a minimum of 50 fibers per image ( $n=3$ images per fiber formulation).

\section{Probiotic stability in PEO and PVA fibers}

In addition to the assessment of post-spin viability and morphology, the temperature stability of $L$. acidophilus formulated in different PEO or PVA fibers (electrospun in water, MRS broth, MRS broth with $5 \%$ glycerol, or with lyophilized

L. acidophilus in MRS broth with/without $5 \%$ glycerol) was tested by CFU counting on MRS agar plates, as described above. Fibers containing L. acidophilus were stored in sealed Petri dishes at either $-20^{\circ} \mathrm{C}, 4^{\circ} \mathrm{C}$, or $25^{\circ} \mathrm{C}$, and probiotic viability was evaluated up to 90 days post-electrospinning. After $1,3,7,30$, and 90 days, a fiber piece ( 2 to $5 \mathrm{mg}$ ) was cut, weighed, dissolved, and incubated in $1 \mathrm{~mL} M R S$ broth for $30 \mathrm{~min}$ on a shaker at $37^{\circ} \mathrm{C}$ and $150 \mathrm{rpm}$. The dissolved fiber solution was then diluted using 10-fold serial dilutions, $2 \mu \mathrm{L}$ was plated on MRS agar plates, and CFUs were counted after $48 \mathrm{hr}$ of anaerobic incubation at $37^{\circ} \mathrm{C}$.

\section{Inhibitory potential of $L$. acidophilus fibers in soluble $G$. vaginalis co- cultures}

To initially determine the ability of $L$. acidophilus fibers to inhibit $G$. vaginalis growth, free $L$. acidophilus and $L$. acidophilus fibers were first co-cultured in media with $G$. vaginalis As described above, the selectivity of co-culture medium for $L$. acidophilus and G. vaginalis growth was determined prior to experiments by 
measuring the growth of free L. acidophilus and G. vaginalis over 48 and $72 \mathrm{hr}$, respectively in either MRS or NYC III broth ${ }^{353,354}$. From these experiments, it was observed that $G$. vaginalis was unable to grow in MRS broth, while L. acidophilus grew in both MRS and more slowly in NYC III. Furthermore, to assess the viability of $G$. vaginalis and $L$. acidophilus in each media at the specific concentrations and time points required for co-culture assays, free $G$. vaginalis $\left(10^{8} \mathrm{CFU} / \mathrm{mL}\right)$ or free L. acidophilus ( $10^{7}$ and $10^{8} \mathrm{CFU} / \mathrm{mL}$ ) were added to $10 \mathrm{~mL}$ MRS and $10 \mathrm{~mL}$ NYC III separately, and incubated for $24 \mathrm{hr}$. At 4 and $24 \mathrm{hr}$, each sample was diluted using 10-fold serial dilutions, $2 \mu \mathrm{L}$ was plated on appropriate agar plates, and CFUs were counted after $48 \mathrm{hr}$ of anaerobic incubation at $37^{\circ} \mathrm{C}$.

\section{Inhibitory potential of $L$. acidophilus fibers in HeLa monolayer G. vaginalis co-cultures}

Human cervical cancer cells (HeLa, originally from ATCC, kindly provided by Dr. Kenneth Palmer, University of Louisville) were cultured at $37^{\circ} \mathrm{C}$ in a $5 \% \mathrm{CO}_{2}$ incubator using Dulbecco's Modified Eagle's Medium (DMEM) supplemented with $10 \%$ heat-inactivated $\left(30 \mathrm{~min}, 56^{\circ} \mathrm{C}\right)$ fetal bovine serum. The culture medium was changed every $48 \mathrm{hr}$ to maintain the cells. HeLa cells were plated in 24-well plates $\left(150,000\right.$ cells/well) and incubated overnight at $37^{\circ} \mathrm{C}$ in a $5 \% \mathrm{CO}_{2}$ incubator to create a confluent cell monolayer. The next day, $1 \times 10^{7}$ or $1 \times 10^{8} \mathrm{CFU} / \mathrm{mL}$ of $L$. acidophilus or $L$. acidophilus fiber containing equivalent dose, and $1 \times 10^{8} \mathrm{CFU} / \mathrm{mL}$ of $G$. vaginalis were co-cultured simultaneously with HeLa cell monolayers. Control groups, including no treatment, blank fibers, free L. acidophilus only, and free G. vaginalis only, were added in parallel to HeLa monolayers. Treatment and control 
plates were then incubated for either 4 or $16 \mathrm{hr}$ at $37^{\circ} \mathrm{C}$ in $5 \% \mathrm{CO}_{2}$ incubator. HeLa cells were checked under the microscope before and after incubation to ensure their viability. After incubation, the media was removed, and non-adherent bacteria were removed by washing each well with $500 \mu \mathrm{L}$ sterile $1 \mathrm{X}$ PBS twice. HeLa cells were lysed by adding $1 \mathrm{~mL}$ of sterile water and incubating for $30 \mathrm{~min}$ at $37^{\circ} \mathrm{C}$ in a $5 \% \mathrm{CO}_{2}$ incubator. The resulting cell suspensions were then collected, serial diluted, and plated on the appropriate agar plates to determine CFU counts. Inhibition of $G$. vaginalis viability was determined as the log-decrease in expression, relative to the CFU count obtained for free G. vaginalis-HeLa sample groups, at each corresponding time point.

\section{Statistical analysis}

All of the in vitro experiments were performed in triplicate, with 3 replicates per sample and the results were shown in average \pm standard deviation. Statistical analyses of samples for fiber morphology, loading, and in vitro assays were performed using one-way ANOVA with the Tukey post hoc test $(P \leq 0.05)$. Statistical analyses of $G$. vaginalis titers were performed using the Mann-Whitney test using Graph Pad Prism version 8.4.3.

\section{Results}

\section{Preliminary preclinical assessment of PEO fibers to deliver vaginal therapies}

To first test the feasibility of utilizing PEO fibers in a mouse model of infection, we assessed the vaginal administration of fibers in $\mathrm{C} 57 \mathrm{BI} / 6 \mathrm{~J}$ mice

vaginally infected with $G$. vaginalis or PBS vehicle control (Figure 4.1A). Data 
comparing H\&E stained fixed tissue sections from control mice (No fibers (EFs); $n=10$ ) and those receiving fibers (fibers (EFs); $n=10$ ) suggested that fibers alone did not markedly alter the vaginal mucosa (Figure 4.1B and Figure 4.S1). The vaginal epithelium remained protected by a layer of keratinized epithelial cells (typical of estrogenized mice) and the squamous epithelium appeared intact. Further, robust polymorphonuclear (PMN) cell infiltration into the epithelium or the stroma were not observed. In line with previous observations, no PMN recruitment into the epithelium was observed in G. vaginalis-infected animals $+/$ - fibers ( $n=5$ per group; Figure 4.1B). Administration of fibers did not promote G. vaginalis infection; mice administered blank PEO fibers had nearly identical $G$. vaginalis titers as mice that were administered no treatment (Figure 4.1C). While further studies in other model systems are necessary, these data preliminarily suggest that PEO fibers are inert to the vaginal mucosa in uninfected and G. vaginalisinfected C57B/6J mice. 
A

कृ.? $\beta$-est

G.v.

$\beta$-est EFs or vehicle

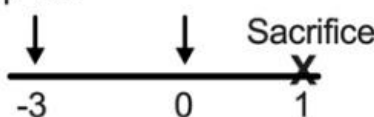

Vaginal wash CFU \& histology

C

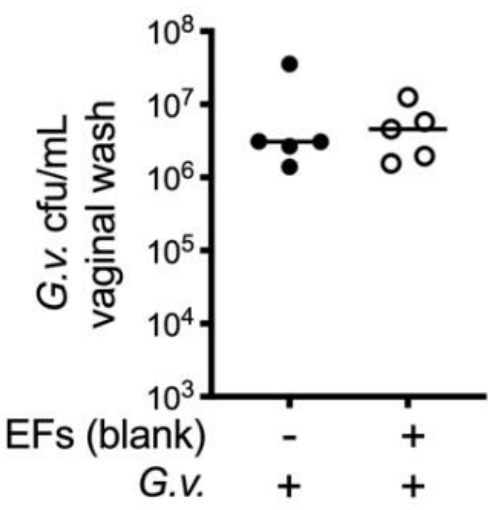

B

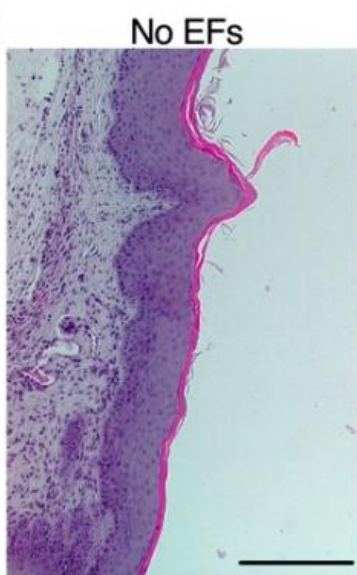

No EFs + G.v.

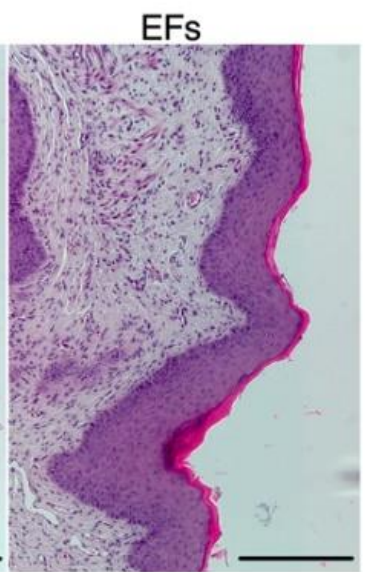

$\mathrm{EFs}+$ G.v.

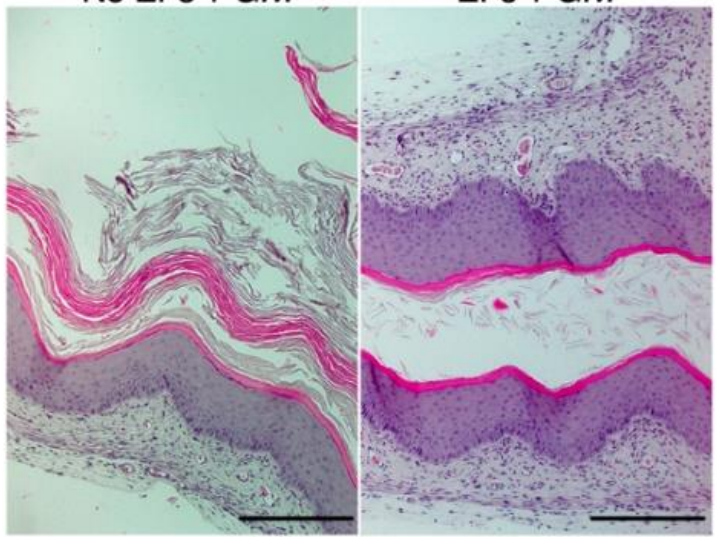

Figure 4.1. PEO fibers (EFs) are safely delivered in the vagina. (A) Blank PEO fiber discs were inserted vaginally into estrogenized C57BI/6J mice infected with PBS (top row, panel B) or G. vaginalis (G.v., bottom row, panel B) at the same time point (0). Twenty-four hr later, vaginal washes were collected, and mice were sacrificed to collect vaginas for histology. (B) Vaginas were fixed, processed and sections stained with hematoxylin and eosin (H\&E). Scale bars $=200 \mu \mathrm{m}$. Images are representative of 10 No EFs, $10 \mathrm{EFs}, 5 \mathrm{No} E F s+$ G.v. and $5 \mathrm{EFs}+$ G.v. mice. See Figure S1 for additional images. (C) G.v. titers in vaginal washes. Each dot represents an individual mouse. 


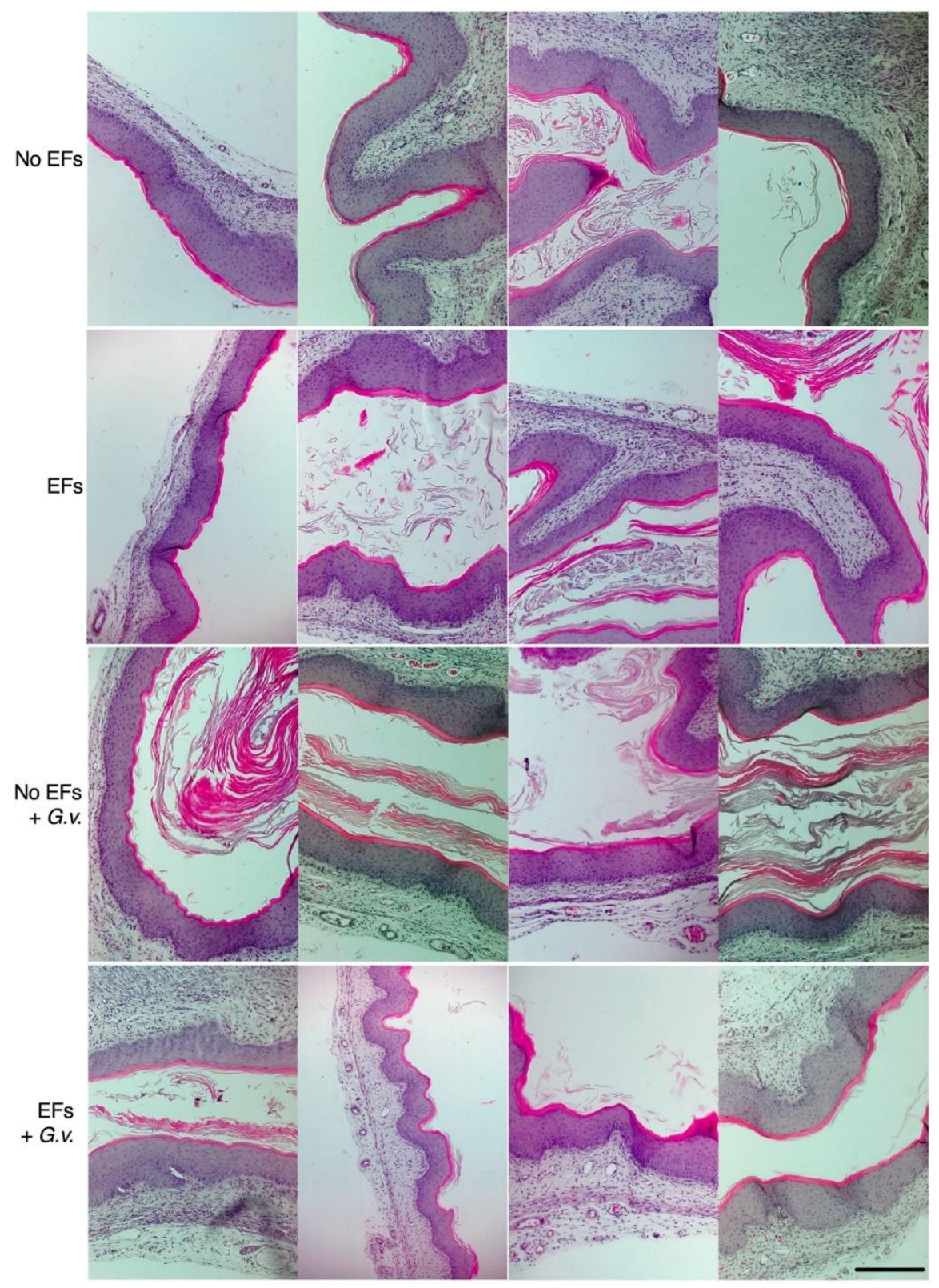

Figure 4.S1. Histological analysis of mouse vaginal tissue. Vaginas were fixed, processed and sections stained with hematoxylin and eosin (H\&E). Scale bar = $200 \mu \mathrm{m}$.

To begin to assess the potential therapeutic benefit of fiber delivery with a current standard therapy, we fabricated PEO fibers loaded with the first-line BV 
therapeutic metronidazole (met). Met-containing PEO fibers, successfully loaded with $828 \pm 72 \mu \mathrm{g}$ met/mg fiber, were evaluated for their ability to prevent and to treat G. vaginalis vaginal infection in mice. In the "prevention" experimental group, met-containing PEO or blank PEO control fibers, were administered immediately prior to $\mathrm{G}$. vaginalis inoculation (Figure 4.2A), while in the "treatment" group, metcontaining or blank PEO fibers were administered $24 \mathrm{hr}$ after G. vaginalis inoculation (Figure 4.2B).

The administration of met-containing PEO significantly reduced $G$. vaginalis infection in both experimental arms. In the prevention arm, the administration of met-containing fibers resulted in a significant decrease of nearly 3-logs in $\mathrm{CFUs} / \mathrm{mL}$, relative to the administration of blank PEO fibers (Figure 4.2C). Similarly, in the treatment arm, mice that received met-containing PEO fibers had $>500$-fold lower G. vaginalis titers after $24 \mathrm{hr}$ treatment (48 hpi) than mice that received blank fibers (Figure 4.2D, right panel). This difference was not due to differences in initial colonization levels between the two groups of mice; $G$. vaginalis levels were indistinguishable at $24 \mathrm{hpi}$ immediately prior to fiber administration (Figure 4.2D, left panel). Finally, whereas 8 of 10 mice that received blank PEO fibers had uterine G. vaginalis above the limit of detection (dotted line), only 4 of 10 mice that received met-containing PEO fibers had uterine $G$. vaginalis above the limit of detection (Figure 4.2E). Together, these results suggest that fibers can effectively deliver therapy to reduce vaginal $G$. vaginalis infection in the context of prevention and treatment. Overall these preliminary studies suggest that 
PEO fibers are able to safely deliver active agents shown to be capable of reducing G. vaginalis infection.
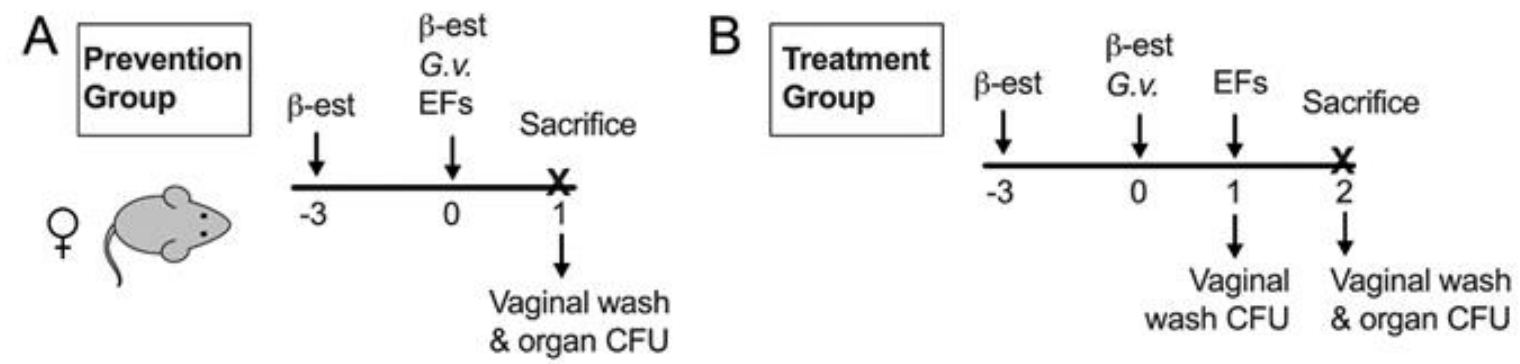

\section{Prevention Group}

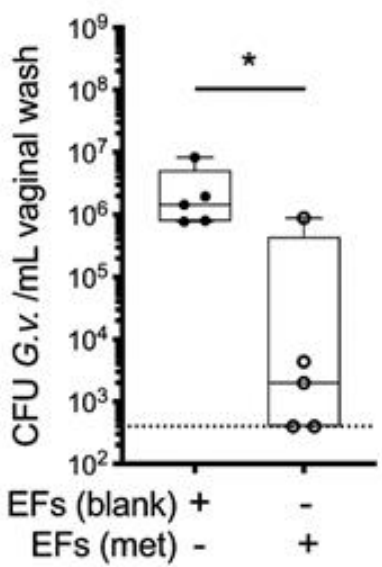

E \begin{tabular}{ccc|}
$\begin{array}{c}\text { Uterine } \\
\text { infection }\end{array}$ & $8 / 10$ & $4 / 10$ \\
\hline
\end{tabular}

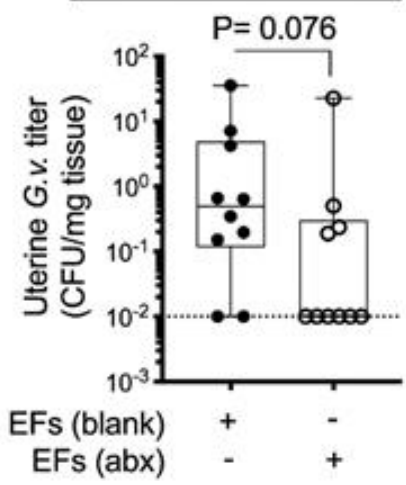

D Treatment Group

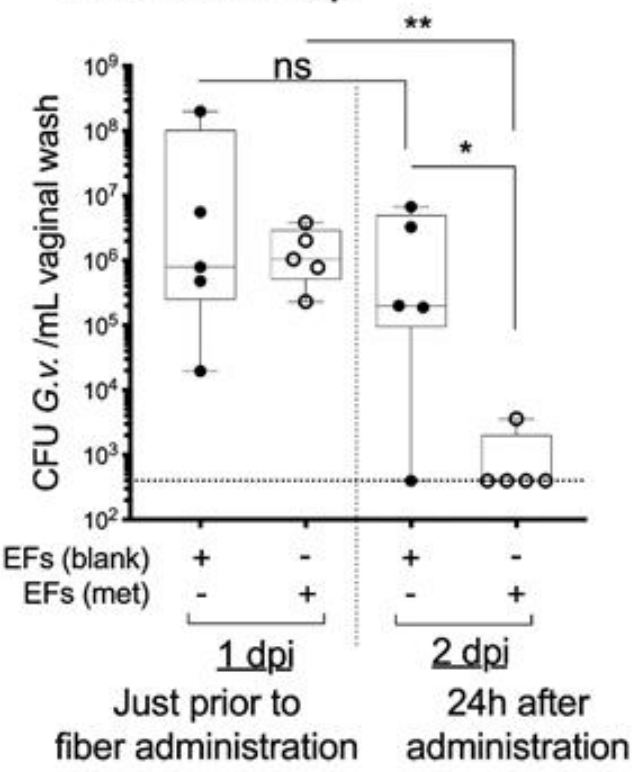


Figure 4.2. Evaluation of the ability of metronidazole-containing PEO fibers (EFs) to exert a therapeutic effect in a mouse model of BV using vaginal inoculation of G. vaginalis. (A) For the prevention arm of the experiment, at time point zero, mice were administered $6 \mathrm{~mm}$ PEO discs, with and without metronidazole, and were immediately intravaginally infected with G.v. Vaginal washes and tissues were collected $24 \mathrm{hr}$ later. (B) In the treatment arm, mice were inoculated with G.v. at time zero, and $24 \mathrm{hr}$ later were administered fibers with or without metronidazole. Vaginal washes were collected immediately prior to fiber insertion, and $24 \mathrm{hr}$ after fiber administration (48 hpi). Vaginal wash titers are shown for both (C) prevention and (D) treatment experiments. (E) G. vaginalis titers in uterine horn tissue from both experimental arms. In panels C-D, each data point represents one mouse. For samples from which $G$. vaginalis was not detectable, the detection limit was plotted (dotted lines). ${ }^{*} \mathrm{P}<0.05,{ }^{* *} \mathrm{P}<0.01$, ns $=$ not significant by Mann-Whitney test.

\section{Probiotic viability in PEO and PVA fibers}

To develop these fibers for probiotic administration, the viability of $L$. acidophilus in electrospun PEO and PVA fibers was first compared with the loading measured prior to electrospinning $\left(5 \times 10^{7} \mathrm{CFU} / \mathrm{mg}\right)$, as a function of probiotic freshness, polymer choice, and electrospinning solution (Figure 4.3). Overall, high probiotic viability on the order of $1.70 \times 10^{7}$ and $2.26 \times 10^{7} \mathrm{CFU} / \mathrm{mg}$ fiber was achieved with the incorporation of fresh $L$. acidophilus in PEO and PVA fibers, respectively. In comparison, the incorporation of lyophilized bacteria decreased the overall viability to 4.40 to $7.20 \times 10^{2} \mathrm{CFU} / \mathrm{mg}$ and 1.30 to $2.60 \times 10^{4} \mathrm{CFU} / \mathrm{mg}$, 
for PEO and PVA fibers, respectively. For the majority of formulations, fibers electrospun with fresh $L$. acidophilus had significantly higher $L$. acidophilus viability relative to fibers electrospun with lyophilized L. acidophilus $(P \leq 0.05)$, demonstrating as much as a 4 to 5 -log increase in L. acidophilus viability in fibers that contained fresh $L$. acidophilus. The only exceptions were for PEO and PVA fibers electrospun in MRS and MRS with glycerol, respectively. In comparison, no statistical significance in L. acidophilus viability was observed between otherwise similar PEO and PVA fiber formulations, as a function of polymer choice (PEO or PVA) or electrospinning solution $(P>0.05)$. 


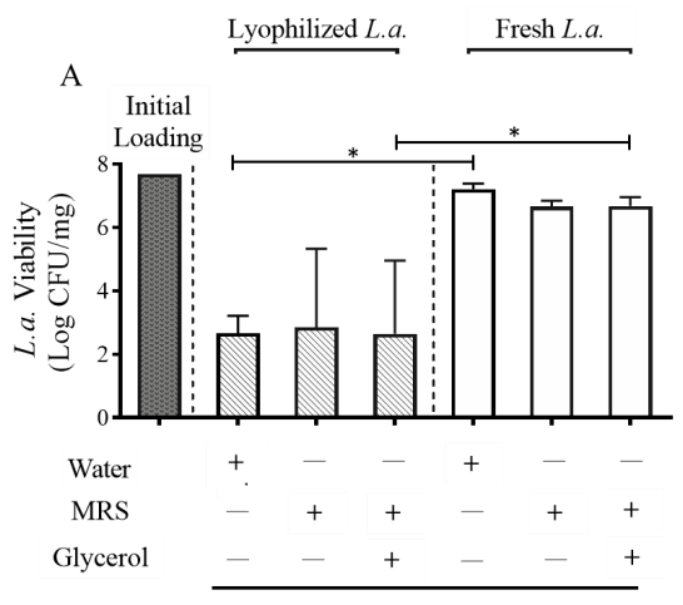

PEO Fiber Post-Spun Loading

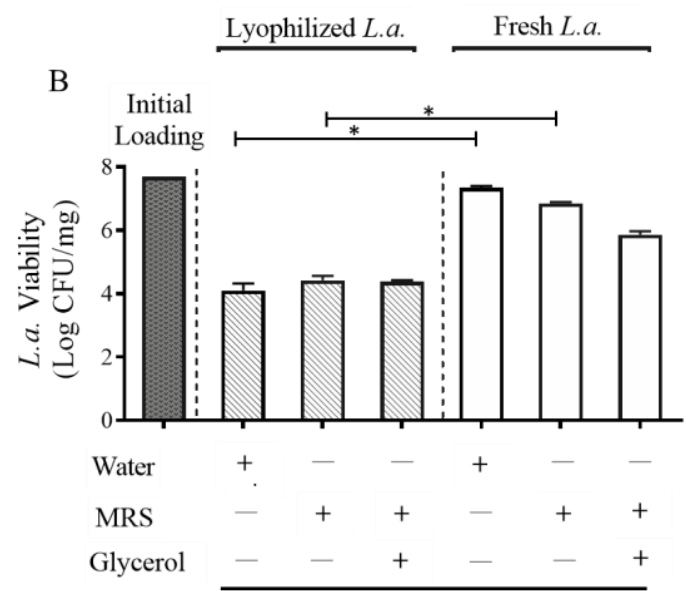

PVA Fiber Post-Spun Loading

Figure 4.3. L. acidophilus (L.a.) viability in different (A) PEO and (B) PVA formulations after electrospinning. The concentration of L.a. aliquoted to the polymer solution prior to electrospinning $\left(5 \times 10^{7} \mathrm{CFU} / \mathrm{mg}\right.$ polymer $)$, was determined via CFU counts, and was considered as $100 \%$ viability. Viability values are shown as the mean \pm standard deviation from three independent fiber batches. Statistical significance between groups, as calculated by one-way ANOVA, is represented by ${ }^{*} \mathrm{P} \leq 0.05$.

\section{Probiotic fiber morphology and diameter}

The morphology of PEO and PVA fibers, electrospun with an intermediate concentration of fresh or lyophilized $L$. acidophilus $\left(5 \times 10^{7} \mathrm{CFU} / \mathrm{mg}\right)$ was evaluated to assess the impact of polymer type, probiotic lyophilization, and electrospinning solution on fiber morphology (Figure 4.S2). As a function of polymer type, PEO fibers possessed well-defined morphologies (Figure 4.S2A-F), while PVA fibers resulted in thicker, more melded morphologies (Figure 4.S2G-L). An uneven distribution of $L$. acidophilus and more pronounced fiber beading were observed in 
some PVA fibers (Figure 4.S2H and I), which was attributed in part to difficulty in electrospinning PVA in aqueous, relative to organic solutions, obscuring the visualization of fresh and lyophilized probiotics in these images. Additionally, the incorporation of fresh bacteria in both PEO and PVA fibers (Figure 4.S2A-C and G-I), regardless of electrospinning solution, seemed to result in more uniform fiber morphology, while fibers electrospun with lyophilized L. acidophilus exhibited increased beading, less consistent morphology, and fewer bacteria per area (Figure 4.S2D-F and J-L). Lastly, as a function of solute type, both water and MRS broth provided feasible solutions for electrospinning PEO and PVA fibers, resulting in well delineated and reproducible fiber morphologies. However, when glycerol was incorporated into the electrospinning solution, fibers seemed to meld together, resulting in less discrete fiber structures (Figure 4.S2C, F, I, L). 


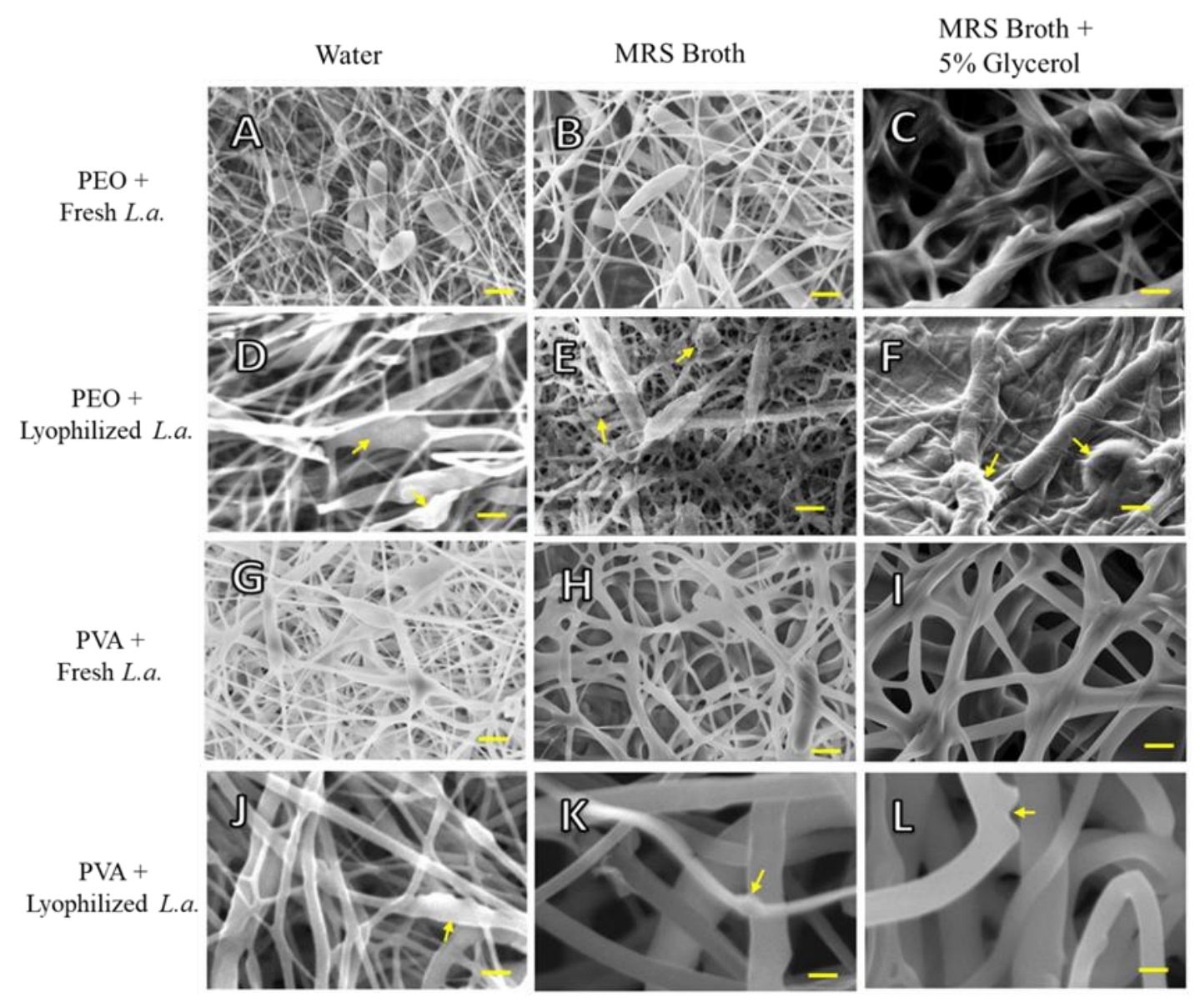

Figure 4.S2. SEM images of $5 \% \mathrm{w} / \mathrm{v}$ PEO and $10 \% \mathrm{w} / \mathrm{v}$ PVA electrospun fibers containing $5 \times 10^{7} \mathrm{CFU}$ L.a./mg polymer: (A through F) 5\% PEO fiber formulations; (G through L) 10\% PVA fiber formulations. Formulations electrospun with (A) - (C) and $(\mathrm{G})-(\mathrm{I})$ fresh L.a. and $(\mathrm{D})-(\mathrm{F})$ and $(\mathrm{J})-(\mathrm{L})$ lyophilized L.a. Fibers electrospun in (A), (D), (G), (J) water; (B), (E), (H), (K) MRS broth; and (C), (F), (I), (F) MRS with $5 \%$ glycerol. Arrows point to incorporated probiotics and scale bars represent $2 \mu \mathrm{m}$.

The diameters of PEO and PVA fibers electrospun with an intermediate concentration of $L$. acidophilus ( $5 \times 10^{7} \mathrm{CFU} / \mathrm{mg}$ ) were measured to assess the impact of different electrospinning parameters and conditions on fiber diameter 
(Figures 4.52 and 4.S3). Overall, PEO fibers had smaller diameters, with a tighter range of distribution between $270 \pm 90$ and $693 \pm 460 \mathrm{~nm}$ compared to PVA fibers with diameters ranging from $400 \pm 210$ to $2136 \pm 71 \mathrm{~nm}$ that were electrospun with similar conditions (Figure 4.S3). Furthermore, no statistical significance in fiber diameter was observed between PEO and PVA fibers electrospun in similar solutes that were loaded with fresh $L$. acidophilus $(P>0.05)$. However, PVA fibers loaded with lyophilized L. acidophilus in MRS with and without glycerol had significantly larger diameters than similarly spun PEO fibers $(P \leq 0.05)$. Additionally, no differences in fiber diameter were observed among PEO fibers electrospun, with either fresh or lyophilized L. acidophilus $(P>0.05)$; however, some PVA fibers containing lyophilized $L$. acidophilus demonstrated larger fiber diameters relative to similar formulations that contained fresh $L$. acidophilus $(\mathrm{P} \leq$ 0.05). Last, electrospinning solution had minimal impact on fiber diameter, with the exception of fresh L. acidophilus PEO fibers, which had a significantly larger diameter when electrospun in MRS and glycerol (versus water, $\mathrm{P} \leq 0.05$ ). Overall, PEO fibers incorporating fresh L. acidophilus that were electrospun in water or MRS broth had the smallest mean diameter $(270 \pm 90 \mathrm{~nm})$, while PVA fibers that were electrospun in MRS and glycerol with lyophilized probiotic had the largest fiber diameter $(2136 \pm 71 \mathrm{~nm})$. 


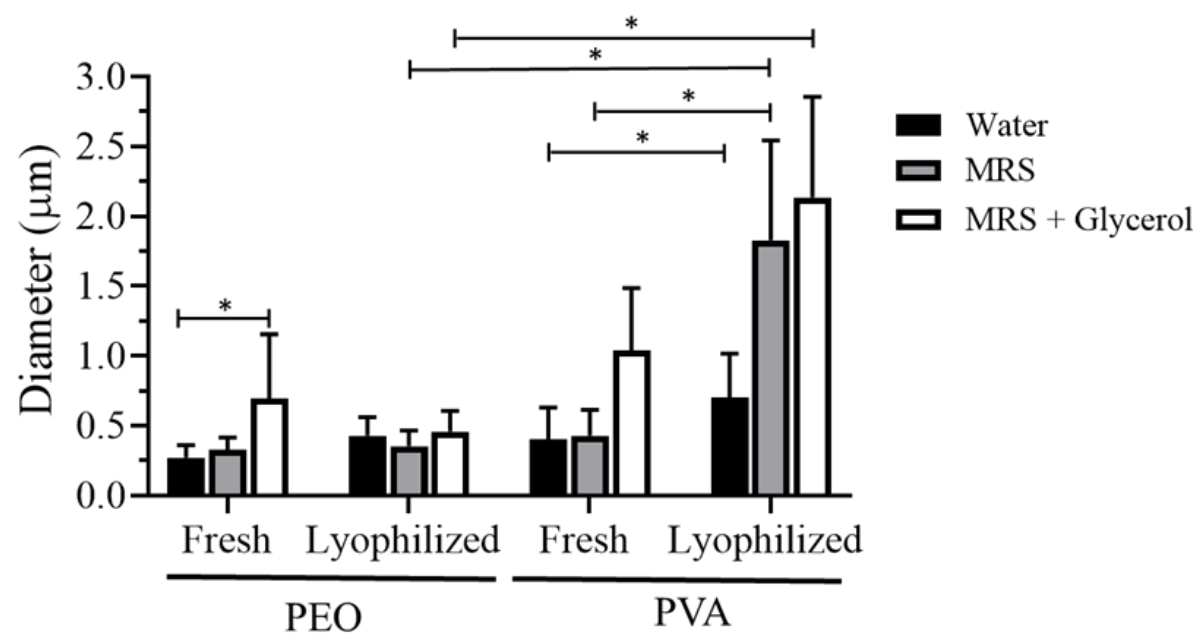

Figure 4.S3. The diameters of PEO and PVA fibers electrospun with $5 \times 10^{7}$ $\mathrm{CFU} / \mathrm{mg}$ fresh or lyophilized L.a. electrospun in water, MRS broth or MRS broth and glycerol. Statistical significance between groups, as calculated by one-way ANOVA, is represented by ${ }^{*} \mathrm{P} \leq 0.05$.

To evaluate the impact of probiotic loading on fiber morphology, PEO and PVA fibers were electrospun in water with different concentrations of fresh $L$. acidophilus ( $5 \times 10^{6}$ to $5 \times 10^{8} \mathrm{CFU} / \mathrm{mg}$ polymer, Figure 4.4). Overall, increased probiotic loading resulted in more rod-shaped regions throughout both PEO and PVA fibers and both PEO and PVA fibers formulations demonstrated well-defined fiber morphology, independent of probiotic loading concentration. However, PVA fibers that were electrospun with the maximum theoretical probiotic loading ( $5 \mathrm{x}$ $10^{8}$ CFU L. acidophilus $/ \mathrm{mg}$ ), exhibited increased beading, consistent with challenges experienced in electrospinning viscous PVA solutions. However, no significant changes in fiber diameter were observed with an increase in probiotic concentration for PEO and PVA fibers electrospun with water and fresh $L$. acidophilus ( $\mathrm{P}>0.05$, Figure 4.4). 

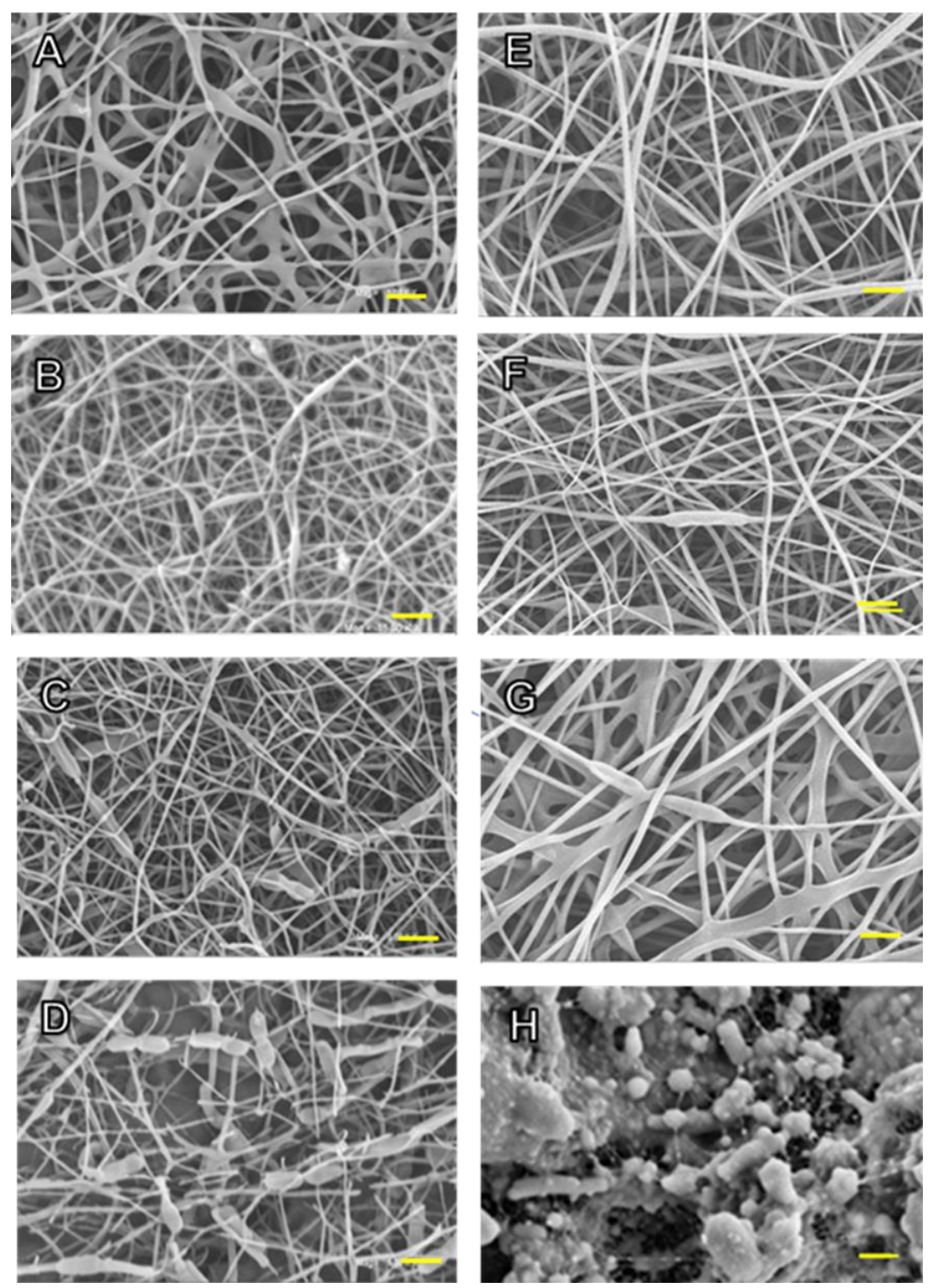

Figure 4.4. SEM images of $5 \% \mathrm{w} / \mathrm{v}$ PEO and $10 \% \mathrm{w} / \mathrm{v}$ PVA fibers that were electrospun in water and contain different concentrations of fresh L.a. (A) Blank PEO fiber and PEO fibers theoretically loaded with (B) 5x106, (C) 5x107, and (D)

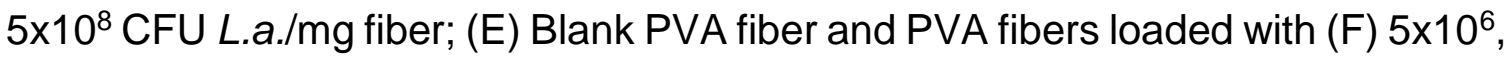
(G) $5 \times 10^{7}$, and (H) $5 \times 10^{8} \mathrm{CFU}$ L.a./mg fiber. Scale bars represent $2 \mu \mathrm{m}$. 


\section{Probiotic stability in PEO and PVA fibers}

To determine the storage stability of PEO and PVA fibers, the long-term viability of L. acidophilus was evaluated at room temperature $\left(25^{\circ} \mathrm{C}\right), 4^{\circ} \mathrm{C}$, and $-20^{\circ} \mathrm{C}$ storage conditions. Multiple formulations were tested using fresh $L$. acidophilus at a concentration of $5 \times 10^{7} \mathrm{CFU} / \mathrm{mg}$, in PEO and PVA fibers that were electrospun in water, MRS broth, or MRS broth with 5\% glycerol (Figure 4.S4). The highest postspun viability during long-term storage (90 days) was observed when fibers were stored at either $4^{\circ} \mathrm{C}$ or $-20^{\circ} \mathrm{C}$, regardless of the polymer and solute type. In contrast, when fibers were stored at room temperature, the viability of $L$. acidophilus was dependent on both the polymer type and electrospinning solution. Within room temperature conditions, L. acidophilus viability in the water-based PEO and PVA formulations rapidly decreased, with no viability seen by day 7 . In contrast, PEO and PVA fibers that were electrospun in MRS broth maintained L. acidophilus viability at room temperature for up to 30 and 7 days, respectively. The addition of glycerol to the MRS broth, decreased the duration of viability to levels seen with water. These data indicate that -20 and $4^{\circ} \mathrm{C}$ are the optimal storage conditions to maintain L. acidophilus viability (in the current formulations), independent of polymer type and electrospinning solution. 

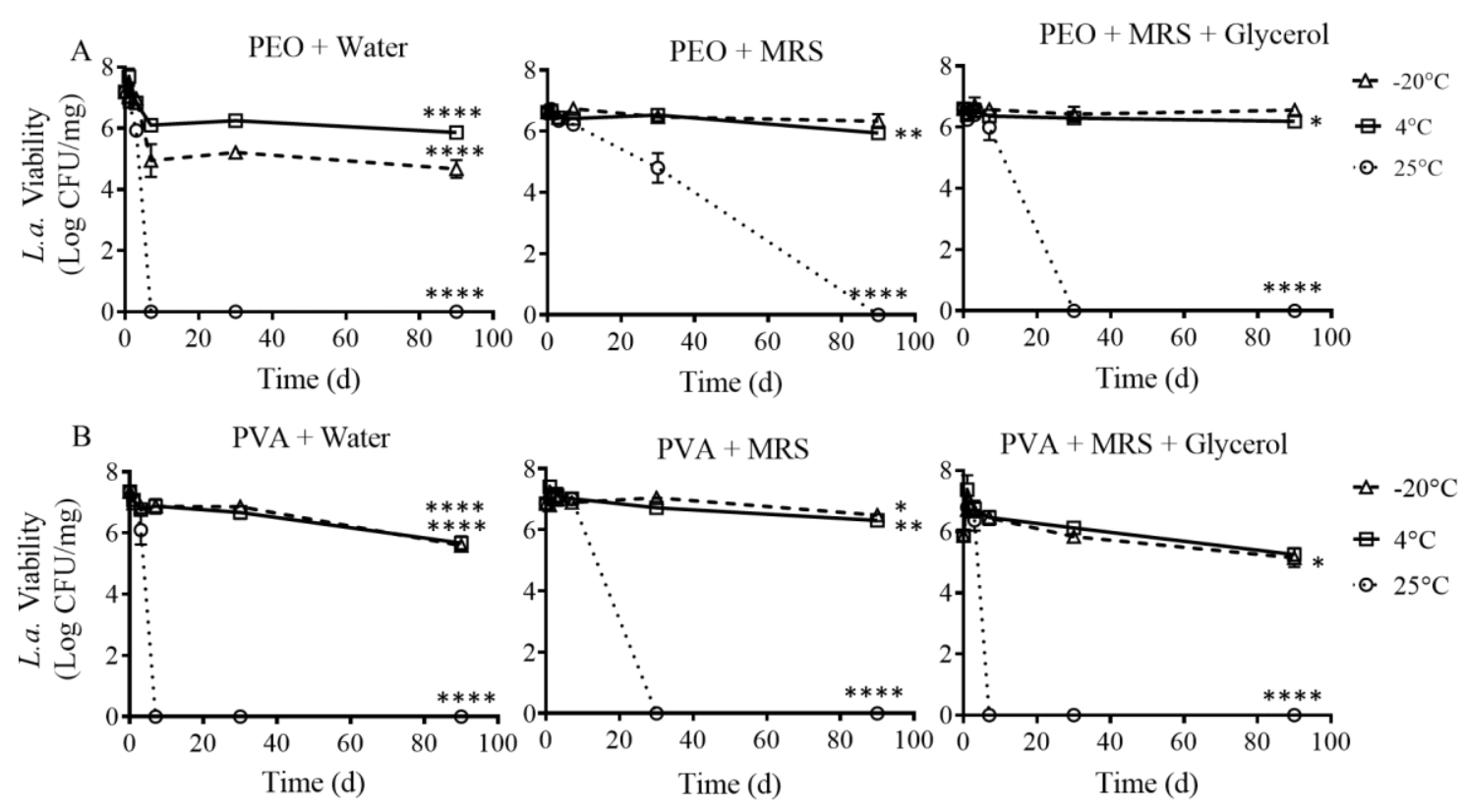

Figure 4.S4. The viability of L.a. in (A) PEO and (B) PVA fibers electrospun with 5 x 107 CFU L.a./mg in water, MRS broth, or MRS broth with 5\% glycerol, was evaluated after storage in $-20^{\circ} \mathrm{C}, 4^{\circ} \mathrm{C}$, and $25^{\circ} \mathrm{C}$ for up to 90 days after electrospinning. Viability values are shown as the mean \pm standard deviation from three independent fiber batches. Statistical significance between groups after 90 days, as calculated by one-way ANOVA, is represented by ${ }^{*} P \leq 0.05,{ }^{* *} P \leq 0.01$, and $^{* * * *} \mathrm{P} \leq 0.0001$.

\section{Inhibitory potential of $L$. acidophilus fibers in soluble $G$. vaginalis co- cultures}

Soluble co-culture experiments were conducted to evaluate the impact of $L$. acidophilus fibers on G. vaginalis viability (Figure 4.5). To ensure appropriate culture conditions, the selectivity of $L$. acidophilus and $G$. vaginalis growth was first evaluated on various agar plates (Table 4.1). Overall, MRS agar was selective for L. acidophilus growth, while NYC III media supplemented with streptomycin was 
selective for G. vaginalis growth. In addition, L. acidophilus and G. vaginalis growth was evaluated in MRS and NYC III broth (without antibiotic) for 48 and $72 \mathrm{hr}$, respectively. In MRS broth, both $L$. acidophilus and $G$. vaginalis growth approached the stationary phase $\sim 32 \mathrm{hr}$ post-inoculation, with high L. acidophilus growth $\left(\mathrm{OD}_{600}=4.36\right)$ and significantly diminished $G$. vaginalis growth $\left(\mathrm{OD}_{600}=\right.$ 0.62 , Figure 4.S5, $\mathrm{P} \leq 0.05)$. When NYC III media was used, L. acidophilus growth in the stationary phase was slightly lower than G. vaginalis growth in the same conditions $\left(\mathrm{OD}_{600}=0.54\right.$ and 1.71, respectively). Overall, the resulting growth curves demonstrate the ability of $L$. acidophilus to grow in both MRS and NYC III broths, albeit with lesser growth in NYC III, while G. vaginalis showed somewhat, but significantly higher growth in NYC III media $(P \leq 0.05)$. Lastly, to evaluate the effect of broth on bacteria viability for the specific concentrations and time frames used in co-culture experiments, the viability of free G. vaginalis $\left(10^{8} \mathrm{CFU} / \mathrm{mL}\right)$ or free L. acidophilus $\left(10^{7}\right.$ and $\left.10^{8} \mathrm{CFU} / \mathrm{mL}\right)$ in either MRS or NYC III broth was evaluated for up to $24 \mathrm{hr}$. Even at these higher starting concentrations, similar decreases in G. vaginalis growth were observed in MRS, relative to NYC III broth, after 4 and $24 \mathrm{hr}(P \leq 0.05)$, while no inhibitory effects were observed for $L$. acidophilus in either broth (Figure 4.S6). These results are similar to those observed in previous work ${ }^{355}$ and validated appropriately conservative cell culture conditions of using NYC III broth for co-culture assays. 

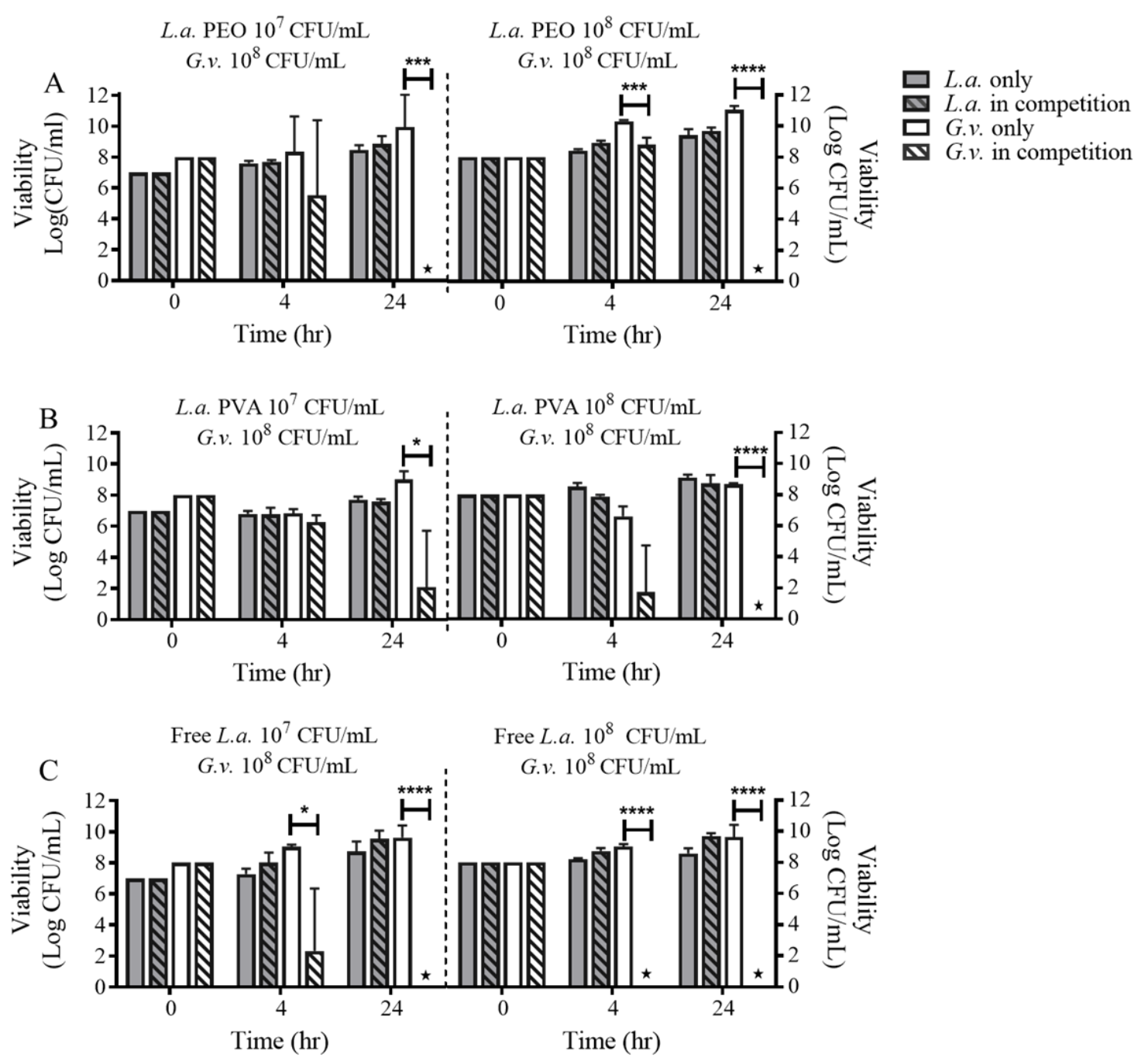

Figure 4.5. L.a. incorporated in PEO and PVA fibers inhibits G.v. viability in soluble co-culture assays. (A) L.a. PEO fibers, (B) L.a. PVA fibers, or (C) free L.a. were co-cultured at one log less (left) or equal (right) concentration relative to G.v. The viability of L.a. or G.v. alone, or as a result of co-culture is noted in the legend. G.v. viability significantly decreased after administration of both tested concentrations of L.a. fibers or free L.a. Columns with zero viability (or viability below the limit of plate-based detection) are represented with a star, and viability values are shown as the mean \pm standard deviation from three independent batches. Statistical 
significance between the viability of free G.v. and G.v. in competition, as calculated by one-way ANOVA, is represented by ${ }^{*} \mathrm{P} \leq 0.05$ and ${ }^{* * *} \mathrm{P} \leq 0.0001$.

Table 4.1. G. vaginalis and $L$. acidophilus growth on different selective agar.

\begin{tabular}{|l|l|c|}
\hline Organisms & Selective Agar & Growth \\
\hline L. acidophilus (ATCC 4356) & MRS agar (Sigma-Aldrich) & Yes \\
\hline L. acidophilus (ATCC 4356) & $\begin{array}{l}\text { NYC III agar with streptomycin (Recipe, Dr. } \\
\text { Lewis, WUSTL) }\end{array}$ & None \\
\hline G. vaginalis (clinical isolate JCP8151B) & MRS agar (Sigma-Aldrich) & None \\
\hline G. vaginalis (clinical isolate JCP8151B) & $\begin{array}{l}\text { NYC III agar with streptomycin (Recipe, Dr. } \\
\text { Lewis, WUSTL) }\end{array}$ & Yes \\
\hline
\end{tabular}
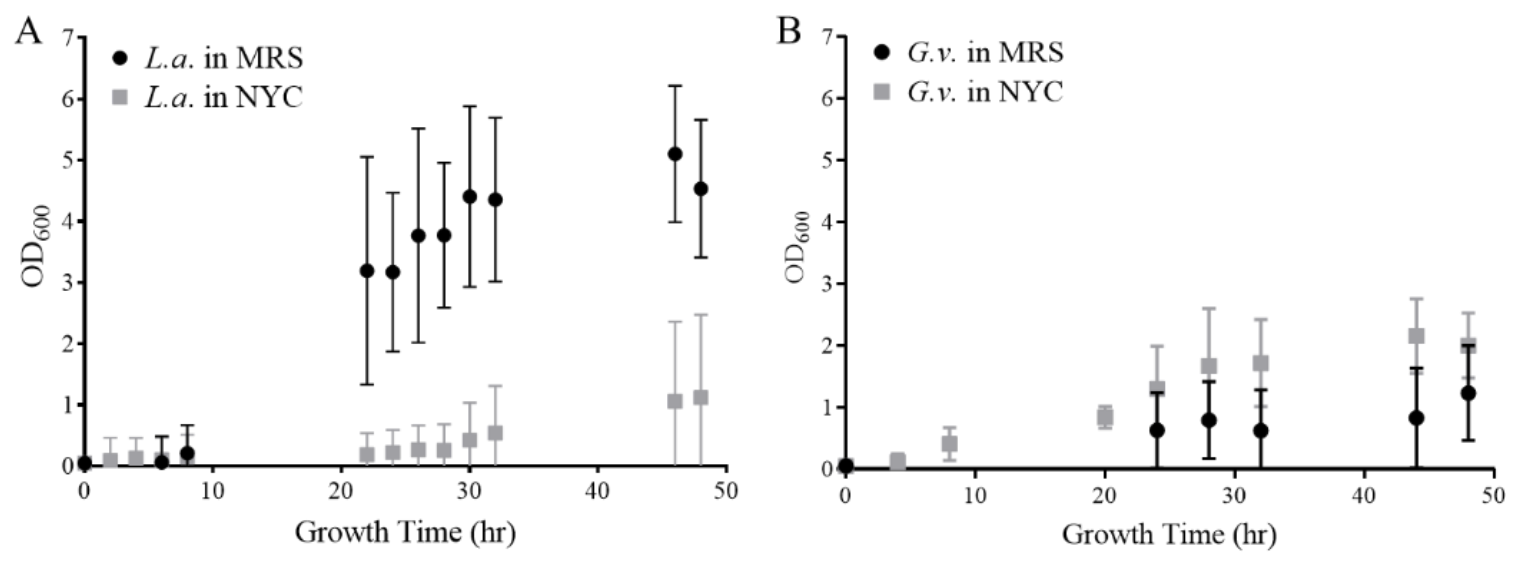

Figure 4.S5. L.a. and G.v. growth in NYC or MRS broth. All bacterial cultures were inoculated from a starting seed culture, which were each sub-cultured to an OD600 of 0.05 . The OD 600 was read every $4 \mathrm{hr}$. Three replicate cultures were grown in 10 $\mathrm{mL}$ of either MRS or NYC III broth in conical tubes. Each time-point sample was taken and analyzed by taking the average of triplicate samples. 


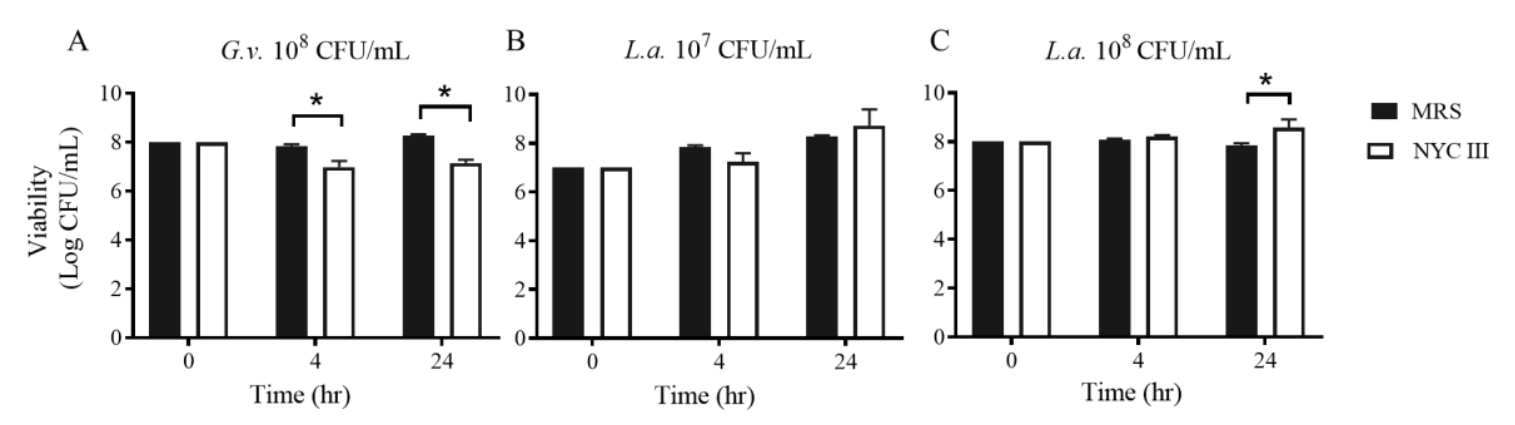

Figure 4.S6. G.v. and L.a. viability after culture in either MRS or NYC III broth. (A) $10^{8} \mathrm{CFU} / \mathrm{mL}$ of G.v., (B) $10^{7} \mathrm{CFU} / \mathrm{mL}$ of L.a., and (C) $10^{8} \mathrm{CFU} / \mathrm{mL}$ of L.a. were inoculated in MRS and NYC III broth and the viability at inoculation, $4 \mathrm{hr}$, and 24 hr post-inoculation were evaluated. MRS slightly inhibited G.v. growth, while no inhibitory effects were observed for L.a. when either broth was used. Statistical significance between groups, as calculated by one-way ANOVA, is represented by ${ }^{*} \mathrm{P} \leq 0.05$.

In the soluble co-culture assay, the effect of $L$. acidophilus dosage form (fiber-incorporated vs. free) and concentration ( $10^{7}$ vs. $\left.10^{8} \mathrm{CFU} / \mathrm{mL}\right)$ was evaluated on G. vaginalis inhibition. L. acidophilus PEO fibers at $10^{7}$ and $10^{8} \mathrm{CFU} / \mathrm{mL}$, demonstrated a partial inhibitory effect on G. vaginalis viability (2.8 and 1.5-logs, respectively) $4 \mathrm{hr}$ post-inoculation (although L. acidophilus PEO fibers at $10^{7}$ CFU/mL were not statistically significant), followed by complete inhibition of $G$. vaginalis (9.9 and 11.1-logs) $24 \mathrm{hr}$ post-inoculation (Figure 4.5A). Similarly, L. acidophilus PVA fibers at $10^{7}$ and $10^{8} \mathrm{CFU} / \mathrm{mL}$, demonstrated a modest inhibitory effect after $4 \mathrm{hr}$ (0.6 and 4.9-logs) and nearly complete or complete inhibition after $24 \mathrm{hr}$ as a function of increased dosing (7 and 8.7-logs, Figure 5B). Free $L$. 
acidophilus at $10^{7}$ and $10^{8} \mathrm{CFU} / \mathrm{mL}$, demonstrated strong inhibition of $G$. vaginalis (6.7 and 9.0-logs) after $4 \mathrm{hr}$, and complete inhibition (9.0 and 9.6-logs) after $24 \mathrm{hr}$ (Figure 4.5C). Blank PEO and PVA fibers were used as negative controls for inhibition and demonstrated no inhibitory effects when co-cultured with $G$. vaginalis

\section{Inhibitory potential of $L$. acidophilus fibers in HeLa monolayer G. vaginalis co-cultures}

Similar experiments were conducted to evaluate the impact of $L$. acidophilus fibers on G. vaginalis co-cultured with confluent HeLa cell monolayers (Figure 4.6). L. acidophilus fibers or free $L$. acidophilus were incubated with $G$. vaginalis and HeLa cells at $37^{\circ} \mathrm{C}$ in a $5 \% \mathrm{CO}_{2}$ incubator for up to $16 \mathrm{hr}$ to ensure HeLa cell viability in the presence of bacteria. Administration of low and high doses (equivalent to $10^{7}$ and $10^{8} \mathrm{CFU} / \mathrm{mL}$ L. acidophilus) of PEO L. acidophilus fibers resulted in negligible changes to $G$. vaginalis viability after $4 \mathrm{hr}(0.83-\log$ increase and 1.1-log increase, $P>0.05)$, with complete inhibition observed (6.9 and 5.8logs, respectively) after $16 \mathrm{hr}$ (Figure 4. 6A). Similarly, $10^{7}$ and $10^{8} \mathrm{CFU} / \mathrm{mL}$ of $L$. acidophilus PVA fibersdecreased $G$. vaginalis viability by 1.7 and 1.5 -logs, respectively after $4 \mathrm{hr}$, followed by complete inhibition after $16 \mathrm{hr}$ (both 8 -logs, Figure 4.6B). In comparison, administration of free L. acidophilus $\left(10^{7} \mathrm{CFU} / \mathrm{mL}\right)$ for $4 \mathrm{hr}$ resulted in partial inhibition of G. vaginalis (1.7-log), while after $16 \mathrm{hr}$, G. vaginalis viability was completely inhibited. However, at the higher dose of free $L$. acidophilus $\left(10^{8} \mathrm{CFU} / \mathrm{mL}\right), \mathrm{G}$. vaginalis viability was completely inhibited as early as $4 \mathrm{hr}$ post-co-culture (Figure $4.6 \mathrm{C}$ ). 

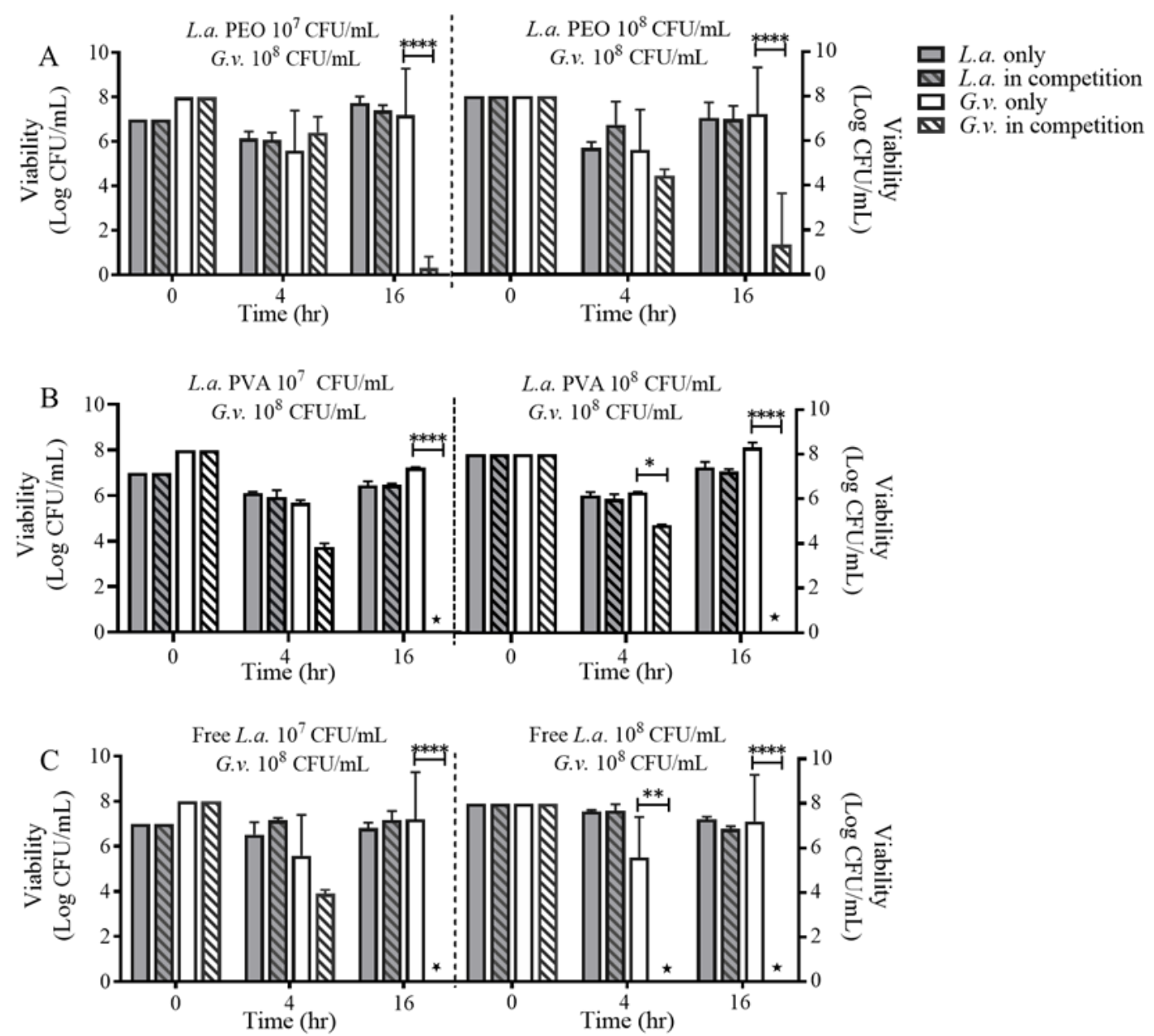

Figure 4.6. L.a. incorporated in PEO and PVA fibers inhibit G.v. growth in epithelial cell co-culture competition assays. (A) L.a. PEO fibers, (B) L.a. PVA fibers, or (C) free L.a. were co-administered with G.v. to HeLa cell monolayers. Columns with zero viability (or viability below the limit of plate-based detection) are represented with a star, and viability values are shown as the mean \pm standard deviation from three independent batches of fibers or free bacteria. Statistical significance between the viability of free G.v. and G.v. in competition, as calculated by one-way ANOVA, is represented by ${ }^{*} P \leq 0.05,{ }^{* *} P \leq 0.01$, and ${ }^{* * * *} P \leq 0.0001$. 


\section{Discussion}

Lactobacilli play an important role in modulating the vaginal environment, and contribute to overall female reproductive health by fostering an environment that is less amenable to pathogenic bacteria adhesion and growth. Numerous in vitro and in vivo studies have shown that the administration of different Lactobacillus species/strains can improve vaginal health as a primary or adjunct treatment to BV, and can outcompete the growth of facultative pathogens including G. vaginalis, Prevotella anaerobius, and Prevotella bivia ${ }^{356,357}$. In addition, studies have shown that species such as $L$. crispatus CTV-05 can inhibit G. vaginalis association with cervicovaginal epithelial cells ${ }^{358}$ and may be more broadly applicable to decreasing the adhesion of uropathogenic organisms to vaginal epithelial cells. Among different lactobacillus species, L. acidophilus has shown strong adhesion to epithelial cells ${ }^{359}$ and has demonstrated success in treating BV both in vivo and in clinical studies ${ }^{329,330}$. Due to these characteristics, L. acidophilus was selected for incorporation in electrospun fibers as a novel alternative delivery platform to inhibit BV infection.

In parallel with the consideration and application of probiotic species for vaginal applications, a variety of vehicles have been developed for probiotic delivery. Currently, orally- or intravaginally-administered probiotic dosage forms exist, but often result in transient effects, requiring frequent administration regimens to be efficacious in the time frame surrounding infection. In particular, several platforms, including gels, films, and capsules, have been developed to

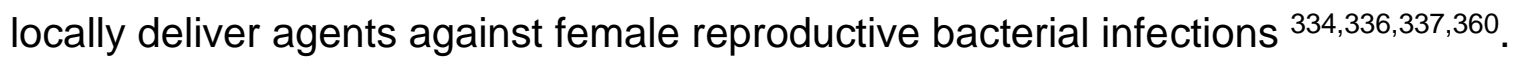


However, some challenges exist with traditional "drug" delivery formulations in that they may be less amenable to the incorporation of live cells, such as probiotics. Furthermore, even with once or twice daily application, BV recurrence persists, emphasizing the need for new dosage forms that can provide on-demand delivery and a foundation upon which to develop sustained-release capabilities to more significantly impact the course of BV infections.

Electrospun fibers have demonstrated versatility and efficacy in preventing and treating intravaginal virus infections ${ }^{341,361,362}$, stimulating interest in their application as a new intravaginal dosage form against bacterial infections. In addition to their versatility in incorporating a variety of active agents with different properties, electrospun fibers incorporate key features including ease-ofmanufacturing, mechanical flexibility, enhanced encapsulant stability, and tunable release of active agents. Recent work has demonstrated the incorporation of probiotics in electrospun fibers for a variety of applications ${ }^{343,347,363-365}$, and has the potential to impact female reproductive health in a multitude of ways by providing localized delivery, maintaining normal vaginal $\mathrm{pH}$, and enabling prolonged release of probiotics or active agents to prevent recurrence. While previous studies have shown success in incorporating live bacteria relevant to biomedical ${ }^{347}$, food $^{347,363,364}$, and female reproductive health applications ${ }^{342,343}$, to our knowledge, there are no studies to date that have evaluated the efficacy of probiotic fibers against BV or other female reproductive bacterial infections.

To first assess the feasibility of our approach for the envisioned application, we wanted to examine the preliminary safety and functional impact of fiber 
administration. There are several important factors to consider. First, it is important that the fibers themselves do not disrupt the vaginal mucosa or trigger tissue inflammation and recruitment of immune cells. Our results suggest that the fibers were relatively inert in the mouse vagina and did not adversely affect the tissue. This finding is promising and provides preliminary evidence regarding the safety of these fibers. However, it is important to acknowledge that these tests were performed in mice injected with $\beta$-estradiol, which is known to have effects on inflammatory processes. Therefore, further safety testing outside of the context of estrogenization, and also at later time points, is still needed. A second factor to consider is whether the fibers themselves promote infection by known pathogens. Importantly, we found that blank fibers did not enhance vaginal infection by $G$. vaginalis Finally, for the fiber approach to be useful, it must be capable of delivering effective therapies. We showed that fibers were capable of delivering metronidazole (current standard-of-care antibiotic for BV) at sufficient levels to both prevent and treat G. $v$. infection in a mouse model. Together these data provide a proof-of-concept foundation that supports the feasibility of utilizing fibers for intravaginal therapeutics.

Based on these promising results, both PEO and PVA were selected for probiotic fiber fabrication due to their known biocompatibility, mucoadhesivity, and hydrophilicity for on-demand applications. From an engineering design and feasibility perspective, we first evaluated the effect of different parameters including electrospinning solution, polymer type, and the incorporation of fresh or lyophilized probiotics on post-spin viability. First, we showed that electrospinning 
solution and polymer type have minimal impact on probiotic viability, whereas the choice of fresh or lyophilized bacteria exerted a significant impact on probiotic viability. Overall, the viability of $L$. acidophilus in fibers electrospun with fresh bacteria was $\sim 4$-logs higher $\left(\sim 10^{7} \mathrm{CFU} / \mathrm{mg}\right)$ than fibers that incorporated lyophilized bacteria $\left(\sim 10^{3} \mathrm{CFU} / \mathrm{mg}\right)$, regardless of polymer type. This striking difference may be attributed in part to the lyophilization process, which is known to exert stress on bacteria. Previous studies have shown that removing water from cells can cause changes in protein conformation and cell membrane structure ${ }^{366}$ leading to low viability, while conversely, water remaining post-lyophilization plays an important role in bacteria survival. One study showed that residual water content between 3 to $6 \%$ can enhance the survival of Lactobacillus salivarius during storage. Additionally, it has been shown that the choice of cryoprotectant can have a significant impact on bacteria viability. In this study, we used skim milk and trehalose which, based on previous studies, demonstrated high viability and functionality relative to other protectants ${ }^{367}$. Lastly, rehydration solution and conditions are also important factors in cell recovery after the dehydration process. While previous studies have shown that a slow rehydration procedure (over the course of 7 to 16 days $)$ and at moderate temperatures $\left(15-25^{\circ} \mathrm{C}\right)$ can improve cell recovery ${ }^{368}$, more rapid rehydration may weaken bacteria and increase susceptibility to the applied electric field and shear stress, resulting in lower cell recovery. While our initial viability results provide functional activity within a therapeutic range, in future work lyophilization and rehydration conditions may be optimized to increase probiotic health and recovery. 
Among the different formulations tested in this work, the highest probiotic viability was achieved by incorporating $5 \times 10^{7}$ CFU fresh L. acidophilus per $\mathrm{mg}$ PEO/PVA fibers. The resulting viability values $\left(\sim 10^{7} \mathrm{CFU} / \mathrm{mg}\right)$ were within the anticipated ranges shown in previous work focused on other applications including food engineering ${ }^{347}$ and vaginal drug delivery systems ${ }^{342,343}$; however, viability is known to vary significantly based on factors including loading concentration, polymer composition, and Lactobacillus type. The highest probiotic viability reported in previous studies demonstrated post-spun viability as high as $90 \%$ for $10 \%$ w/w PVA fibers that incorporated fresh L.g. at $2 \times 10^{6} \mathrm{CFU} / \mathrm{mL}$ water ${ }^{347}$. Similarly, a viability as high as $68 \%$ was reported in a study that incorporated fresh L. acidophilus in PVA fibers with the post-spun viability of $1.9 \times 10^{6} \mathrm{CFU} / \mathrm{mg}^{342}$. In contrast, a study that incorporated L. plantarum in PEO fibers for mucosal delivery purposes, obtained only $2 \%$ post-spun viability $\left(4.7 \times 10^{8} \mathrm{CFU} / \mathrm{mL}\right)$ with a theoretical loading of $2.3 \times 10^{10} \mathrm{CFU} / \mathrm{mL}^{369}$, indicating that higher initial loading may decrease probiotic viability. Another study in which 10\% PVA, 20\% PVP, or 45\% w/w PVP solutions were electrospun in water with $3 \times 10^{6} \mathrm{CFU} / \mathrm{mg}$ fresh $L$. acidophilus, obtained $68 \%, 34 \%$, and $40 \%$ L. acidophilus viability in the respective fiber formulations, suggesting the impact of polymer composition on probiotic viability ${ }^{342}$. A study which evaluated the feasibility of encapsulating bacteriocins and lactic acid producing bacteria in PEO fibers, demonstrated cell viability as low as $0.1 \%$ for Escherichia coli and as high as $75 \%$ for Micrococcus luteus cells incorporated, suggesting that smaller gram-positive bacteria such as Micrococcus luteus may be more resistant to high voltages and the electrospinning process ${ }^{369}$. 
Lastly, a recent study that incorporated 10 different fresh Lactobacilli species in 4\% w/w PEO (900 kDa) fibers, achieved viabilities as high as $10^{9} \mathrm{CFU} / \mathrm{mg}$ polymer, but varied based on species (e.g., L.g. $(79 \%)$ and L. acidophilus $(20 \%))^{343}$. In our study, this high level $\left(10^{9} \mathrm{CFU} / \mathrm{mg}\right)$ of probiotic incorporation in fibers was challenging to achieve, possibly due to the differences in formulation of lyophilized vs. fresh bacteria used comparatively and the resulting electrospinning solution viscosity. However, these studies confirm that probiotic viability may be impacted by a multitude of factors including loading concentration, polymer type, electrospinning conditions, and bacterial species.

In addition to probiotic viability, we assessed the effect of different processing conditions including electrospinning solution, lyophilization, and polymer composition on fiber morphology and diameter. In general, electrospinning in water or MRS broth led to well-defined fiber morphologies, relative to fibers spun with MRS and glycerol, which resulted in fiber beading and increased fiber diameters. These observations are in agreement with previous work that showed the addition of $10 \%$ glycerol to $40 \% \mathrm{w} / \mathrm{v}$ zein in $90 \%$ ethanol, doubled the diameter of zein fibers relative to fibers spun without glycerol, and decreased the conductivity of polymer solution while negligibly impacting solution viscosity ${ }^{370}$. We anticipate that the addition of glycerol may have had a similar effect on fiber morphology here, and with no overarching benefit to probiotic viability, may be excluded in future formulations. Another parameter in our study that seemed to result in less uniform morphology and larger fiber diameters was the incorporation of lyophilized, relative to fresh probiotic, which may be attributed 
in part to the use of cryoprotectants during lyophilization, known to alter the viscosity of electrospinning solution. Lastly, while PEO and PVA both resulted in well-defined fibers at low probiotic concentrations, alterations to fiber morphology became more pronounced in PVA fibers as probiotic loading increased. Notably, fiber beading was observed, which may be attributed to changes in the viscosity and conductivity of the polymer solution. Furthermore, at all loading concentrations tested, PEO fibers had smaller diameters and more uniform properties, making PEO formulations more conducive to reproducible spinning. Similar observations have been reported for PEO, relative to PVA fibers that incorporated an antimicrobial agent, showing overall smaller diameters for PEO fibers, and suggesting that even the incorporation of low molecular weight agents (relative to cells) can impact fiber diameter ${ }^{371}$.

In addition to evaluating $L$. acidophilus viability immediately after electrospinning, the stability of $L$. acidophilus viability in fibers was examined at different storage temperatures for up to 90 days. As anticipated, storage temperature was a primary factor that impacted probiotic viability over prolonged durations. When fibers were stored at room temperature, L. acidophilus viability decreased dramatically over the first few days and resulted in negligible viability after one month, regardless of polymer composition or electrospinning solution ( $P$ $\leq 0.0001)$. However, minimal decreases $(0.5$ and $0.1-\mathrm{log})$ in $L$. acidophilus viability were observed in PEO fibers that were electrospun in MRS and MRS with glycerol, respectively after 3 months storage at $-20^{\circ} \mathrm{C}(P>0.05)$. Furthermore, minimal differences in L. acidophilus viability were observed over prolonged durations 
when PVA fibers were stored in $4^{\circ} \mathrm{C}(\mathrm{P}>0.05)$. This increased viability is likely attributed to a lower probiotic metabolism at reduced temperatures, requiring less nutrients for prolonged survival. Based on a comparison of electrospinning solutions, when MRS broth (with or without glycerol) was used for electrospinning, a slight, but statistically non-significant increase in L. acidophilus viability was observed $(P>0.05)$, relative to probiotics electrospun in water, regardless of storage temperature. This may be partially attributed to providing a probiotic nutrient source that remains within the fibers after solute evaporation, and may be investigated more thoroughly in future work via the incorporation of other and more concentrated nutrient sources. Lastly, L. acidophilus maintained slightly, but not statistically significant, higher viability in PEO fibers spun in MRS (with or without glycerol, 0.04- and 0.30-logs reduction in viability), relative to PVA fibers spun with the same conditions (0.72- and 0.38 -logs reduction in viability) over 3 months at $20^{\circ} \mathrm{C}$, indicating that the well-defined morphology of PEO fibers may better protect probiotics from environmental stresses such as temperature and dehydration. Together these results indicate that PEO fibers electrospun in water, MRS, or MRS with glycerol provide similar L. acidophilus stability at 4 or $-20^{\circ} \mathrm{C}$.

While previous studies have incorporated probiotics for food processing and biomedical applications, to our knowledge, probiotic fibers have not yet been investigated as a dosage form, demonstrating efficacy against vaginal bacterial infections in vitro (or in vivo). In these studies, soluble co-culture experiments showed that PEO fibers containing L. acidophilus at $10^{7}$ and $10^{8} \mathrm{CFU} / \mathrm{mL}$ decreased G. vaginalis viability after $4 \mathrm{hr}$ by $\sim 3$ - and 1.5 -log, while corresponding 
concentrations of PVA fibers decreased G. vaginalis viability by $0.6-\log$ and 4.9 log, respectively. After $24 \mathrm{hr}$, PEO fibers completely inhibited G. vaginalis, regardless of concentration, while PVA fibers necessitated a higher dose $\left(10^{8}\right)$ to completely inhibit G. vaginalis Overall these results suggest a time- and dosedependent effect of fibers on $G$. vaginalis viability, that was somewhat more pronounced at the early time point (4 hr) for PVA fibers. These observations are in agreement with a previous study that showed that $G$. vaginalis viability decreased significantly (by 1.3 to 2.4 -logs) after $4 \mathrm{hr}$ co-culture with 7 different strains of lactic acid producing bacteria ${ }^{372}$.

Parallel to soluble co-culture experiments, we evaluated the ability of $L$. acidophilus fibers to prevent G. vaginalis viability (and adhesion to) HeLa cells. In competition assays, PEO and PVA fibers demonstrated therapeutic levels of inhibition of $\mathrm{G}$. vaginalis after $16 \mathrm{hr}$ delivery at both $10^{7}$ and $10^{8} \mathrm{CFU} / \mathrm{mL}$. Overall, within the set of conditions tested here, it seems that fibers may take slightly longer (on the order of hours) to exert an effect on G. vaginalis viability and adhesion. However, these results from $L$. acidophilus fibers are in agreement with previous studies showing that the adhesion of free lactobacilli to epithelial cells limits the adherence and growth of other pathogenic bacteria such as $G$. vaginalis ${ }^{373}$. Some studies have shown that the bactericidal activity of lactobacilli against vaginal pathogens occurs within the first hours of application, while other studies indicate that a longer administration time may be required ${ }^{373}$. In this study, the incorporation of probiotics within fibers may initially provide some level of probiotic localization due to entrapment in the fibers, perhaps preventing or delaying probiotic diffusion 
and corresponding epithelial surface coverage, relative to free L. acidophilus Furthermore, some of the mucoadhesive attributes, which we anticipate will be beneficial in in vivo studies, may be less (or more) evident in the context of in vitro assays. Finally, the adhesion and colonization of probiotics may vary across vaginal cell types, hence we plan to examine these features in vaginal epithelial cells EpiVaginal ${ }^{\mathrm{TM}}$ tissue, and in the more complex in vivo model in near-future studies.

For the first time, this work demonstrates the promising potential that new probiotic fibers may have against bacterial vaginosis. Previous ex vivo and clinical

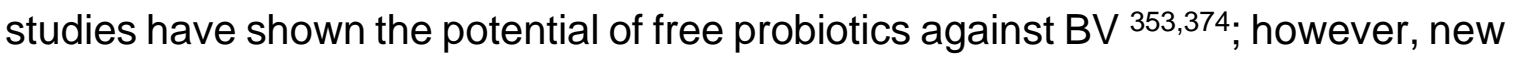
dosage forms are needed to improve current treatment regimens and outcomes. Our in vivo studies suggest that fibers are safe in vivo and can effectively deliver therapeutics in a murine model of $G$. vaginalis infection, In parallel, our in vitro studies show that probiotics are highly and viably incorporated in electrospun fibers, and that prolonged stability of probiotics in electrospun fibers is achieved for up to 3 months, in -20 or $4^{\circ} \mathrm{C}$ storage conditions. Furthermore, PEO and PVA fibers inhibit $G$. vaginalis viability and adhesion to cells suggesting their ability to exert health-promoting effects to treat BV. In parallel work, we are building upon this formulation to create a delivery platform that can sustain probiotic release to offer a potentially new dosage form that may be developed with other attributes. Furthermore, we anticipate that probiotic fibers have the potential to further the existing understanding of the effects of probiotics and delivery vehicles on the 
vaginal microbiome, the host inflammatory response, and BV disease markers and progression. 


\section{CHAPTER 5}

\section{MESH AND LAYERED FIBER ARCHITECTURES AS NOVEL PLATFORMS TO SUSTAIN PROBIOTIC RELEASE AGAINST BACTERIAL VAGINOSIS INFECTION}

\section{Introduction}

Nearly $33 \%$ of women worldwide are afflicted with bacterial vaginosis $(B V)^{375,376}$, a dysbiosis in which Lactobacillus-dominance in the female reproductive tract (FRT) is partially or completely displaced with vaginal pathogens, most prominently Gardnerella vaginalis (G. vaginalis) ${ }^{323,326,377,378}$. Bacterial vaginosis infections are challenging to treat and highly recurrent ${ }^{379}$. Furthermore, BV infection is also associated with significant adverse effects, including increased risk of adverse pregnancy outcomes, preterm labor, postsurgical infections, and the increased acquisition of sexually transmitted infections (STIs) $375,376,380-383$.

The current treatment of BV consists of orally- or intravaginallyadministered antibiotic regimens, comprised of the antibiotics metronidazole, clindamycin, and tinidazole, that have an initially high efficacy ranging from 50$80 \%^{384}$. Despite this initially high efficacy, patients suffer from frequent recurrences and the development of antibiotic-resistant bacteria, as well as other negative 
symptoms including painful sexual intercourse, rectal pain, inflammation, Crohn's disease $^{385}$, and opportunistic yeast infections ${ }^{386}$. To address these challenges, recent research has begun to focus on alternatives, specifically probiotic delivery, to stabilize the vaginal microbiota by providing a Lactobacillus-dominant environment in which to inhibit the growth and adherence of pathogenic bacteria involved in BV. Probiotic approaches have been shown to help treat BV by modulating the endogenous composition of the vaginal microbiota and local $\mathrm{pH}$ through the production of hydrogen peroxide and lactic acid, modulation of host immune responses, and preventing pathogenic bacteria adhesion to epithelial cells $326-328$.

Lactic acid-producing Lactobacillus species such as $L$. crispatus and $L$. acidophilus (have shown non-inflammatory properties and are two of the few species that have shown promise to cure BV ${ }^{384,387}$. Lactic acid, a metabolic byproduct produced by Lactobacillus, promotes Lactobacillus-dominance in the reproductive tract ${ }^{388}$, exerts immunomodulatory and antimicrobial effects against many pathogenic bacteria ${ }^{378,389}$, and acts as a barrier to pathogen colonization (by inducing weak acid stress). L. crispatus is considered to be one of the most predominant, stable, and protective species that promotes vaginal health, by producing a variety of compounds including lactic acid, which in large amounts has been shown to have microbicidal and virucidal properties ${ }^{390}$. Previous clinical studies have shown that both capsules containing low concentrations of $L$. crispatus ( $10^{6}$ to $10^{8}$ colony-forming units (CFU)/capsule) and tampons containing higher concentrations of L. crispatus $\left(2 \times 10^{9} \mathrm{CFU} / \mathrm{dose}\right)$ are safe, and result in 
successful colonization ${ }^{391,392}$. Lactobacillus acidophilus is another extensively explored species that has been associated with a reduced risk of BV. Previous in vitro studies have shown that $L$. acidophilus isolated from the healthy FRT can inhibit a variety of pathogenic bacteria including Bacteroides spp., Prevotella bivia and G. vaginalis, isolated from vaginal swabs of BV-infected women. These impacts are attributed to the high production of hydrogen peroxide, lactic acid, and bacteriocins, and lactic acid which enhance host immune response and inhibit pathogen growth ${ }^{393}$. In addition, a variety of studies have suggested that oral or intravaginal administration of $L$. acidophilus increases lactobacilli colonization in the reproductive tract and restores vaginal flora, relative to administration of a placebo $^{394-396}$.

A very recent approach that has shown potential to treat initial and recurring BV infections, is the use of vaginal microbiome transplantation (VMT) to reestablish the vaginal microbiota ${ }^{397,398}$. A clinical study investigated the impact of VMT on five BV patients and reported that after 1 to 3 VMT sessions, four patients achieved full remission during a 5 to 21 month follow-up period, with no adverse effects $^{399}$. Furthermore, due to the preliminary success of VMT in clinical trials, a universal donor screening protocol was developed to consider donor matching, ethical considerations, and the potential for STI transmission ${ }^{400}$. Based on these early results seen after multiple transient administrations, it may be envisioned that prolonged delivery of diverse patient-specific microbiota populations to the FRT, may be advantageous to provide robust colonization and stabilization of the vaginal microbiota. The promise of VMT highlights the potential that a diverse set 
of patient-derived beneficial species may have on female reproductive health outcomes. However, questions still remain regarding how best to deliver individual or multiple species to meet the long-term delivery needs of women.

Several methods, including gels, capsules, tampons, and liposomes, have been developed to deliver probiotics and other BV treatments to the

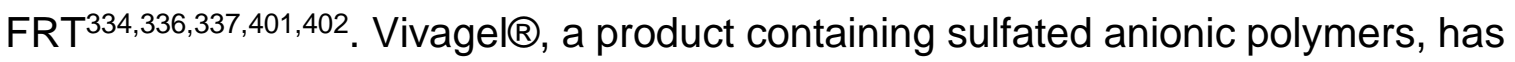
been used to deliver SPL7013, a polylysine dendrimer with anionic sulfate surface functional groups; however, Vivagel® was found to elicit pro-inflammatory cytokine production and polymer-induced toxicity to both epithelial cells and endogenous lactobacilli55. Similarly, for the delivery of lactic acid, gels in combination with antibiotics have shown increased lactobacilli colonization and decreased BV recurrence, relative to the administration of traditional antibiotics alone ${ }^{323,403 ;}$ however, transient delivery and antibiotic resistance remain concerns. In addition, tampons and pessaries have demonstrated low BV cure rates, attributed to decreased quantities of delivered bacteria ${ }^{404}$. Lastly, these dosage forms are generally plagued with low intravaginal residence time; transient protection; messiness via leakage; and unfavorable discharge that can adversely impact user adherence and efficacy ${ }^{155,246,378,405,406}$.

To avoid BV recurrence and address the challenges and inconvenience of daily treatment regimens, a delivery vehicle that provides for sustained probiotic release may be impactful, particularly for infections that require frequent administration regimens. However, to-date, only one dosage form, a pod-based intravaginal ring (IVR), has shown the potential to provide sustained-release of 
probiotics for intravaginal application, with modest daily release of $L$. gasseri as high as $\sim 10^{7} \mathrm{CFU} /$ day for 21 days $^{340,378}$. However, IVRs may be susceptible to pathogenic biofilm formation and yeast adhesion, posing an additional challenge to BV treatment ${ }^{5,407,408}$.

Relative to the currently available dosage forms, electrospun fibers may offer an effective new strategy to deliver probiotics to the FRT'127,129,130,249254,256,260,261,264,409-413. These delivery platforms may extend treatment and prevention applications to BV, as fibers have been shown to enhance encapsulant stability and half-life, lower the doses necessary to achieve efficacy, prolong active agent delivery in the FRT, and provide a scaffold for bacterial residence. Additionally, electrospun fibers provide high surface-area-to-volume ratio ${ }^{411,414}$ and porous morphology, which may be therapeutically envisioned to foster an environment for prolonged probiotic growth and retention ${ }^{415}$. Previous studies have shown that electrospun fibers can incorporate different probiotics with high viability and may be used as a patient-friendly solid-dosage form for intravaginal delivery $342,344,345$. A recent study by our group showed that polyethylene oxide (PEO) fibers formulated alone or with a traditional antibiotic are preliminarily safe and capable of delivering effective therapeutics in an established murine model of BV infection. In addition, this study showed that hydrophilic rapid-dissolve PEO and polyvinyl alcohol (PVA) electrospun fibers incorporating L. acidophilus provided high in vitro viability, stability, and release of $L$. acidophilus Additionally, probiotic fibers completely inhibited $G$. vaginalis viability and cell adhesion in vitro 
in both co-culture and epithelial-G. vaginalis infection, suggesting their feasibility to exert health-promoting effects against BV-associated pathogens ${ }^{416}$.

In this work, we developed novel probiotic fibers with two different fiber architectures, mesh and layered, to locally deliver lactobacilli for prolonged durations ( $2 \mathrm{wk}$ ) (Figure 1). We hypothesized that the incorporation of two model organisms, L. crispatus and L. acidophilus, in fibers may be used as a proof-ofconcept that can be generalized to other Lactobacillus species. First, the changes in the macro- and microstructure of each fiber architecture were evaluated to obtain a better understanding of probiotic integration and fiber degradation. Then, the sustained-release of probiotics from these fibers was evaluated by assessing the cumulative release and proliferation of L. crispatus and L. acidophilus, D- and L-lactic acid production, and changes in $\mathrm{pH}$ over a period of 2 wk. Lastly, within each architecture (e.g., mesh and layered), the formulation with the highest probiotic release was selected to evaluate its efficacy against $G$. vaginalis growth in a soluble co-culture assay. Our results showed that after $48 \mathrm{hr}$ and 6 days, all formulations produced as much as $10^{8}$ and $10^{9} \mathrm{CFU}$ probiotic/mg fiber in total, respectively, with corresponding daily release on the order of $10^{8} \mathrm{CFU} /(\mathrm{mg} \cdot \mathrm{day})$. In addition to high levels of probiotic release, probiotic fibers produced therapeutically-relevant levels of lactic acid, causing a significant reduction in $\mathrm{pH}$. Finally, mesh and layered probiotic fiber dosage forms demonstrated strong inhibition of G. vaginalis, resulting in an 8- and 6.5-log decrease in G. vaginalis viability after $24 \mathrm{hr}$. These promising results suggest that electrospun fibers have the potential to serve as an alternative dosage form by sustaining probiotic and 
lactic acid release and inducing changes relevant to achieving prolonged prevention and treatment of BV.

\section{Materials and Methods}

\section{Bacterial cultures}

Probiotics (L. crispatus MV-1A-US and L. acidophilus 4356) were purchased from American Type Culture Collection (ATCC) and a streptomycinresistant clinical isolate of G. vaginalis (JCP8151B, GenBank JX860320) was generously provided by Dr. Amanda Lewis (Washington University, St. Louis, MO). L. acidophilus and L. crispatus were grown using deMan, Rogosa, and Sharpe (MRS, Sigma 69966) broth and agar plates, both supplemented with $0.1 \%$ Tween 80. G. vaginalis was grown using New York City III (NYC III) broth and agar plates, both supplemented with 10\% heat-inactivated horse serum (Gibco 26050088). The NYC III agar plates contained $1 \mathrm{mg} / \mathrm{mL}$ streptomycin sulfate salt (Sigma S9137) to select for the antibiotic-resistant $G$. vaginalis strain.

Bacterial cultures were initially prepared from frozen stocks using an inoculation loop to streak frozen culture on the appropriate agar plates. Each bacterium was initially cultured on agar plates under anaerobic conditions and maintained at $37^{\circ} \mathrm{C}$ in $5 \%$ hydrogen, $5 \%$ carbon dioxide, and balanced with nitrogen using a Bactronez Anaerobic Workstation (Shel Lab). The incubation time to observe the growth of Lactobacillus species on MRS plates was $48 \mathrm{hr}$, while G. vaginalis cultures were incubated for $72 \mathrm{hr}$ on NYC III agar plates due to slower colony formation. For all bacterial sub-cultures, a single bacteria colony was 
selected from the agar plate and cultured in $1 \mathrm{~mL}$ of MRS or NYC III broth in a closed microcentrifuge tube for 48 or $72 \mathrm{hr}$ at $37^{\circ} \mathrm{C}$, to minimize exposure to air. The bacteria were subsequently sub-cultured in broth by diluting $200 \mu \mathrm{L}$ of $L$. crispatus or L. acidophilus with $9.8 \mathrm{~mL}$ of MRS broth (1:50 dilution) and $20 \mu \mathrm{L}$ of G. vaginalis with $980 \mu \mathrm{L}$ NYC III broth (1:50 dilution). The method of determining the correlation between the $\mathrm{OD}_{600}$ and respective CFU counts was reported previously ${ }^{416}$. One $\mathrm{OD}_{600}$ of $L$. crispatus, L. acidophilus, and G. vaginalis was found to be equivalent to $5.8 \times 10^{7}, 9.2 \times 10^{7}$, and $1.3 \times 10^{9} \mathrm{CFU} / \mathrm{mL}$, respectively.

\section{Fiber synthesis}

PLGA (50:50, 0.55-0.75 dL/g, 31-57 kDa MW) and PEO (600,000 MW) were purchased from Lactel Absorbable Polymers (Cupertino, CA) and Sigma Aldrich (St. Louis, MO), respectively. To fabricate blank fibers, PLGA (15\% w/w) and PEO (5\% w/w) were dissolved in $3 \mathrm{~mL}$ of hexafluoro-2-propanol (HFIP, Fisher Scientific, Waltham, MA, USA) and MRS broth, respectively and were incubated at $37^{\circ} \mathrm{C}$ overnight. For probiotic-containing formulations, $150 \mathrm{mg}$ of PEO w/w was added to $2.5 \mathrm{~mL}$ MRS broth and immediately before electrospinning, $0.5 \mathrm{~mL}$ of MRS incorporating $5 \times 10^{7}$ CFU L. crispatus or L. acidophilus/ mg PEO was added to PEO solution. To minimize the risk of fiber contamination, polymer solutions were passed through a $0.45 \mu \mathrm{m}$ syringe filter (VWR, PA, USA) before probiotic incorporation. In addition, the electrospinning box and all materials that were in contact with fibers were sterilized prior to electrospinning.

During electrospinning, a positive voltage of 18 and $25 \mathrm{kV}$ was applied at the tip of a needle connected to two syringes containing PLGA and PEO, 
respectively. The electrospun fibers were collected on a rotating, $8 \mathrm{~mm}$ outer diameter stainless steel mandrel, positioned $18 \mathrm{~cm}$ from the blunt needle tip of the PLGA syringe and $15 \mathrm{~cm}$ from the needle tip of PEO syringe. The syringe flow rates were maintained at $0.3 \mathrm{~mL} / \mathrm{hr}$ for both PLGA and PEO to fabricate PLGA:PEO (1:1) fibers, while flow rates of 0.1 and $0.3 \mathrm{~mL} / \mathrm{hr}$ were used to fabricate PLGA:PEO (1:3) fibers. For the mesh architecture, the collector was placed in between the PLGA and PEO syringes at a distance of 18 and $15 \mathrm{~cm}$, and fibers were collected simultaneously on the collector. For the layered architecture, a layer of PLGA fibers was electrospun (1 mL) and a PEO layer $(1 \mathrm{~mL})$ was subsequently electrospun on top of PLGA fibers. The schematic design of mesh and layered fibers, as well as the anticipated probiotic delivery mechanism from these platforms to the FRT are shown in Figure 5.1. 

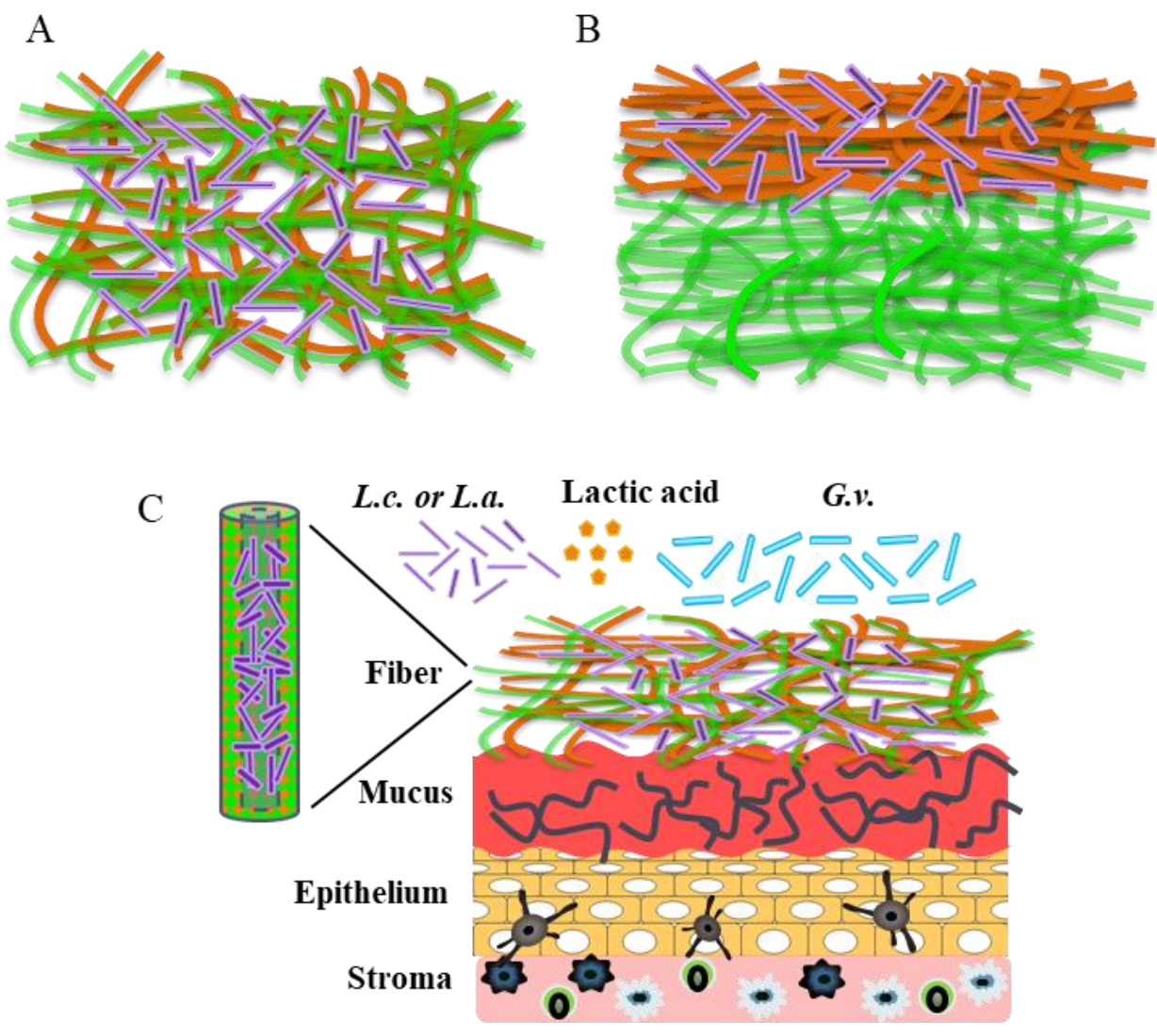

Figure 5.1. Schematic design of (A) mesh and (B) layered fiber architectures. Orange and green fibers represent PEO and PLGA fibers and purple rod-shape structures represent Lactobacillus. (C) Envisioned delivery of probiotic-containing fibers to the vaginal mucosa.

\section{Characterization of fiber morphology and degradation}

The morphology of PEO and PLGA fibers in mesh and layered architectures (with or without probiotic) was characterized using scanning electron microscopy (SEM). Fibers were placed on carbon tape, sputter-coated with a layer of palladium/gold alloy, and imaged using Supra 35 SEM (Zeiss, Oberkochen, Germany). Images were analyzed using ImageJ software and the average fiber 
diameter of each formulation was determined by drawing a line across a minimum of 50 fibers per image ( $n=3$ images per fiber formulation).

The mass loss of blank mesh and layered PLGA:PEO fibers (1:1 and 1:3) was determined over 3 wk by comparing the dry weight remaining at a specific time point with the initial weight. For each fiber formulation, 8 pieces of fibers with predetermined weight ( 8 to $10 \mathrm{mg}$ ) were placed in a $5 \mathrm{~mL}$ centrifuge tube filled with $5 \mathrm{~mL}$ simulated vaginal fluid (SVF, $\mathrm{pH} 4.5)$ and incubated at $37^{\circ} \mathrm{C}$. At each time point (e.g. 1, 8, 24, 48, $72 \mathrm{hr}, 1,2$, and $3 \mathrm{wk}$ ), the fiber was removed and desiccated for 1 wk and weighed to calculate the mass loss.

\section{Quantification of probiotic release and proliferation from fibers}

The release of $L$. crispatus and $L$. acidophilus from, and proliferation of these probiotics in mesh and layered fibers were assessed in MRS broth for 2 wk. Pre-weighed fibers were placed in $5 \mathrm{~mL}$ centrifuge tubes and incubated in $5 \mathrm{~mL}$ MRS broth at $37^{\circ} \mathrm{C}$ with constant shaking at $150 \mathrm{rpm}$. At each time point (e.g., 1, $4,8,24,48,72 \mathrm{hr}, 1,2$, and $3 \mathrm{wk})$, the full volume ( $5 \mathrm{~mL})$ of MRS broth was collected and serially diluted (10-fold dilutions), plated on MRS agar plate, incubated in anaerobic conditions at $37^{\circ} \mathrm{C}$ for $48 \mathrm{hr}$, and then evaluated for CFU counts. After collection, the centrifuge tube was filled with $5 \mathrm{~mL}$ fresh MRS broth.

\section{Quantification of lactic acid production and $\mathrm{pH}$ changes in probiotic fiber eluates}

Fiber release eluates, collected at the corresponding probiotic release time points were used to assess lactic acid production and $\mathrm{pH}$ change resulting from fiber administration. One $\mathrm{mL}$ of each eluate was first centrifuged at $2500 \times \mathrm{g}$ for 5 
min to separate the bacteria from the solution and then the solution was serially diluted (10-fold dilutions). The amount of D- and L-lactic acid was assessed using a lactic acid detection kit (R-biopharma; Darmstadt, Germany) with a sensitivity limit of $1.5 \mu \mathrm{g} / \mathrm{mL}$. The corresponding $\mathrm{pH}$ of eluates at these time points was determined using Fisherbrand ${ }^{\mathrm{TM}}$ plastic $\mathrm{pH}$ strips 5-9 (Fisher Scientific) with an accuracy of $0.5 \mathrm{pH}$ units.

Inhibitory potential of mesh and layered fibers in soluble G. vaginalis cocultures

To evaluate the potential of mesh and layered fibers to inhibit $G$. vaginalis growth, free probiotics and probiotic-containing fibers were co-cultured with $G$. vaginalis in NYC III. For this experiment, the optimal formulation for each architecture was selected based on the highest probiotic release and proliferation observed over 2 wk. Based on results, L. crispatus PEO: PLGA (1:1) was chosen as a model for efficacy studies for both mesh and layered designs due to the high release and structural flexibility.

Briefly, $1 \times 10^{6} \mathrm{CFU} / \mathrm{mL}$ of probiotic or equivalently dosed L. crispatus PEO:PLGA (1:1) mesh and layered fibers $(1.2 \mathrm{mg} / \mathrm{mL})$ were added to $5 \mathrm{~mL}$ microcentrifuge tubes. For free L. crispatus, the bacterial solution was centrifuged $(3000 \times \mathrm{g})$ and the broth was removed, while for L. crispatus fibers, the fiber itself was placed in the tube. Then, $1 \times 10^{6} \mathrm{CFU} / \mathrm{mL}$ of $\mathrm{G}$. vaginalis in NYC III, corresponding to the range of $G$. vaginalis observed during $\mathrm{BV}$ infection $\left(10^{4}\right.$ to $10^{8}$ $\mathrm{CFU} / \mathrm{mL})^{417}$, were added to the tube, followed by the addition of $5 \mathrm{~mL}$ NYC III broth. Fifty $\mu \mathrm{L}$ of the co-culture media was removed after 4,24 , and $48 \mathrm{hr}$ anaerobic 
incubation at $37^{\circ} \mathrm{C}$ and was serially-diluted in 96-well plates by adding $4 \mu \mathrm{L}$ of bacteria solution to $196 \mu \mathrm{L}$ of NYC III broth (1:50 dilutions). The plates were placed on a shaker to ensure complete mixing and $5 \mu \mathrm{L}$ of each sample were plated in triplicate on the appropriate media agar plates to determine the resulting concentration of $L$. acidophilus and G. vaginalis After 24 and $48 \mathrm{hr}$ incubation of MRS and NYC III plates in anaerobic condition $\left(37^{\circ} \mathrm{C}\right)$, the number of colonies for each dilution was counted and converted to $\mathrm{CFU} / \mathrm{mL}$. Control groups included free G. vaginalis, free L. crispatus, and blank fibers (without L. crispatus) cultured in NYC III media. Inhibition of $G$. vaginalis viability was determined as the logdecrease in expression, relative to the free $G$. vaginalis CFU count for that sample group, at each corresponding time point.

\section{Statistical Analyses}

All in vitro experiments were performed 3 independent times with 3 replicates for each sample, and the results are shown as the average \pm standard deviation. Statistical analyses of samples for probiotic release and proliferation, lactic acid release, $\mathrm{pH}$ changes, and in vitro co-culture assays were performed using one-way ANOVA with the Tukey post hoc test $(P \leq 0.05)$ using Graph Pad Prism version 8.4.3.

\section{Results}

\section{Physical Characterization of Mesh and Layered Electrospun Fibers}

First, fluorescently-labeled PLGA:PEO fibers (1:1 and 1:3) were electrospun to visually distinguish and evaluate the ratio of PLGA to PEO fibers 
resulting from the dual-electrospinning process. The resulting mesh PLGA:PEO (1:1 and 1:3) fibers were imaged using fluorescence microscopy (Figure 5.S1), and PLGA fibers (shown in green) were discernible by the larger fiber diameters, relative to PEO fibers (shown in red). Mesh fibers with the same flow rate (1:1), resulted in a 2.5 -fold increase (from 0.2 to 0.5 ) in the green-to-red ratio of PLGA:PEO fibers, relative to fibers dual-spun with a 1:3 PLGA:PEO flow rate.
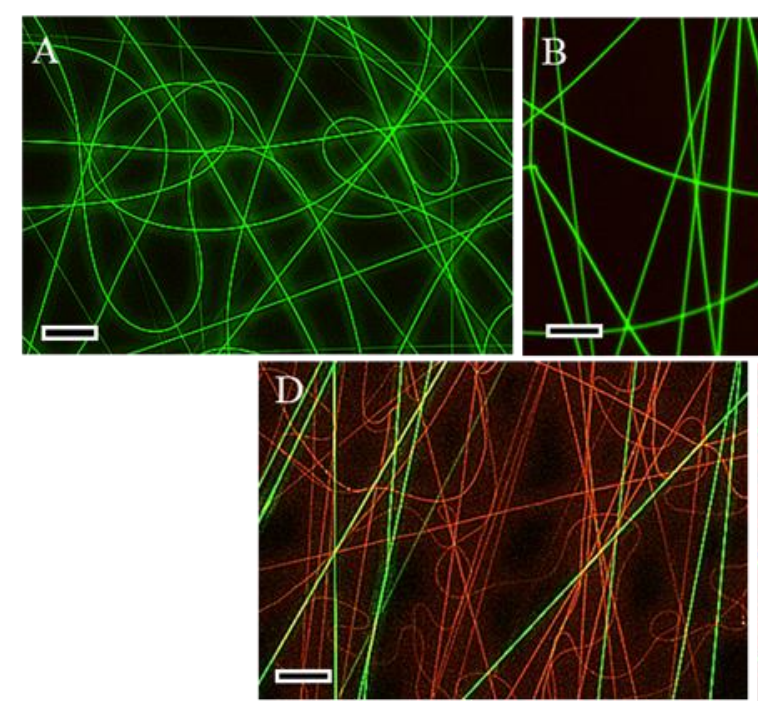
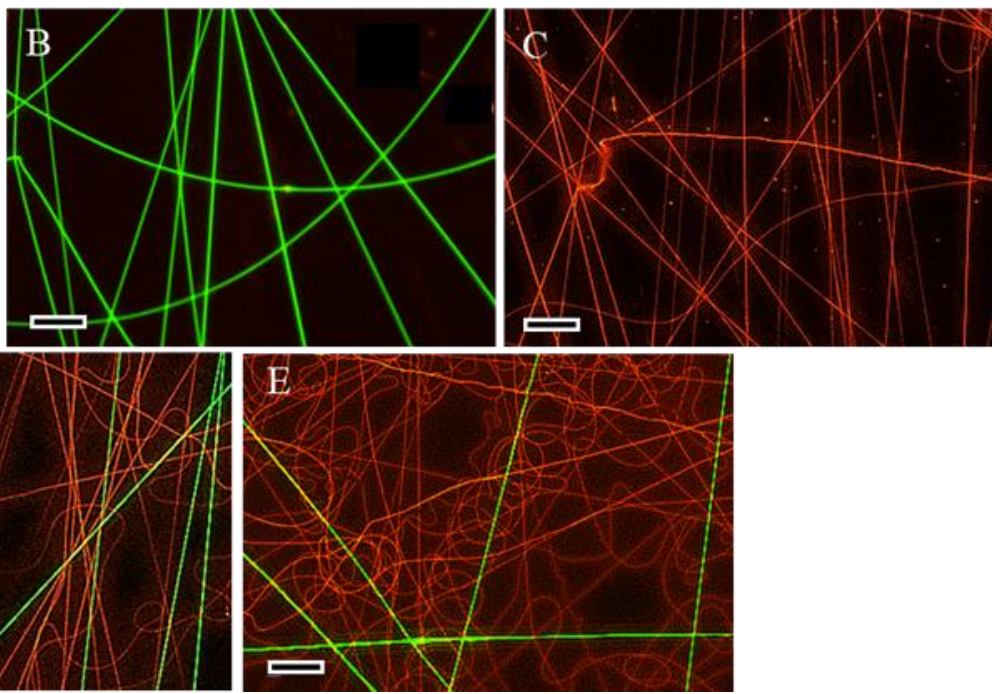

Figure 5.S1. Fluorescence microscopy images of different mesh fiber compositions as a result of dual-electrospinning with different PLGA (green) to PEO (red) flow rate ratios: (A) PLGA $0.3 \mathrm{~mL} / \mathrm{hr}$, (B) PLGA $0.1 \mathrm{~mL} / \mathrm{hr}$, (C) PEO 0.3 $\mathrm{mL} / \mathrm{hr}$, (D) mesh PLGA:PEO (0.3:0.3 mL/hr), and (E) PLGA:PEO (0.1:0.3 mL/hr). Fibers were collected on glass slides after 30 seconds of electrospinning. Image magnification was $40 \mathrm{X}$ and scale bars represent $40 \mu \mathrm{m}$.

The SEM images of blank and probiotic mesh and layered PLGA:PEO (1:1 and 1:3) fiber architectures are shown in Figure 5.2 and Figure 5.S2, respectively. 
Mesh and layered fibers comprised of $15 \mathrm{w} / \mathrm{w} \%$ PLGA in HFIP and $5 \mathrm{w} / \mathrm{w} \%$ PEO in MRS broth resulted in well-defined, uniform, and bead-free fibers. The average diameters of blank mesh architecture PLGA and PEO fibers were 2.16 $\pm 0.31 \mu \mathrm{m}$ and $339 \pm 132 \mathrm{~nm}$, respectively. For blank layered architecture fibers, images were taken from the PEO side, showing uniform and bead-free PEO fibers with a mean diameter of $425 \pm 177 \mathrm{~nm}$. The PLGA side of the layered fibers (data not shown) had an average diameter of $1.33 \pm 0.41 \mu \mathrm{m}$. Furthermore, the uniform morphology of mesh and layered fibers was retained when either L. crispatus or L. acidophilus was incorporated, and rod-shaped structures, highlighted in yellow, were distributed throughout the fibers, forming wider regions in PEO fibers (with an average diameter of $1.10 \pm 0.14 \mu \mathrm{m})$. However, no differences were observed in fiber morphology and diameter as a function of probiotic type (i.e., L. crispatus or L. acidophilus) or flow rate, within each fiber architecture. No alterations to PLGA or PEO fiber diameter and morphology were observed outside of these probioticincorporated regions. 
Mesh Fiber

PLGA:PEO (1:1)
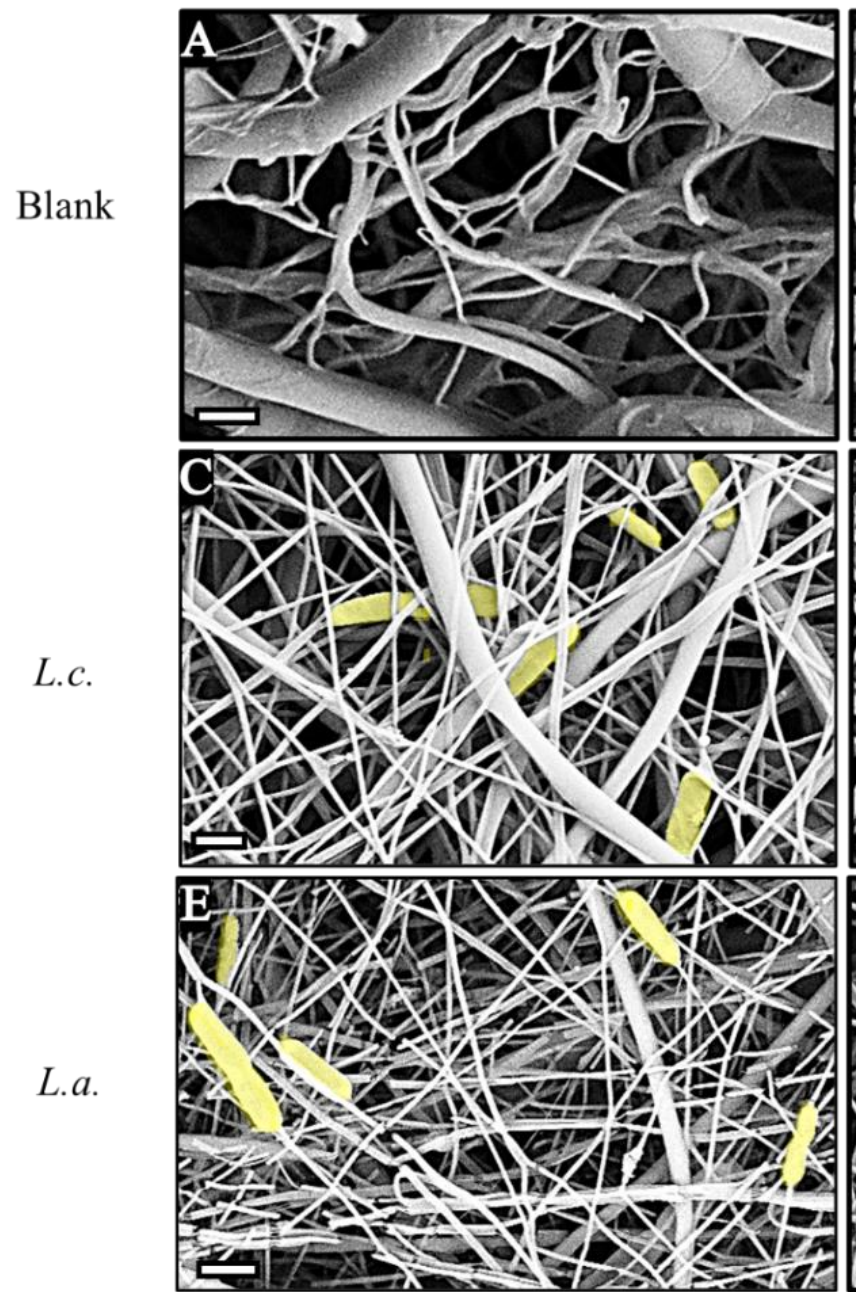

Mesh Fiber

PLGA:PEO (1:3)
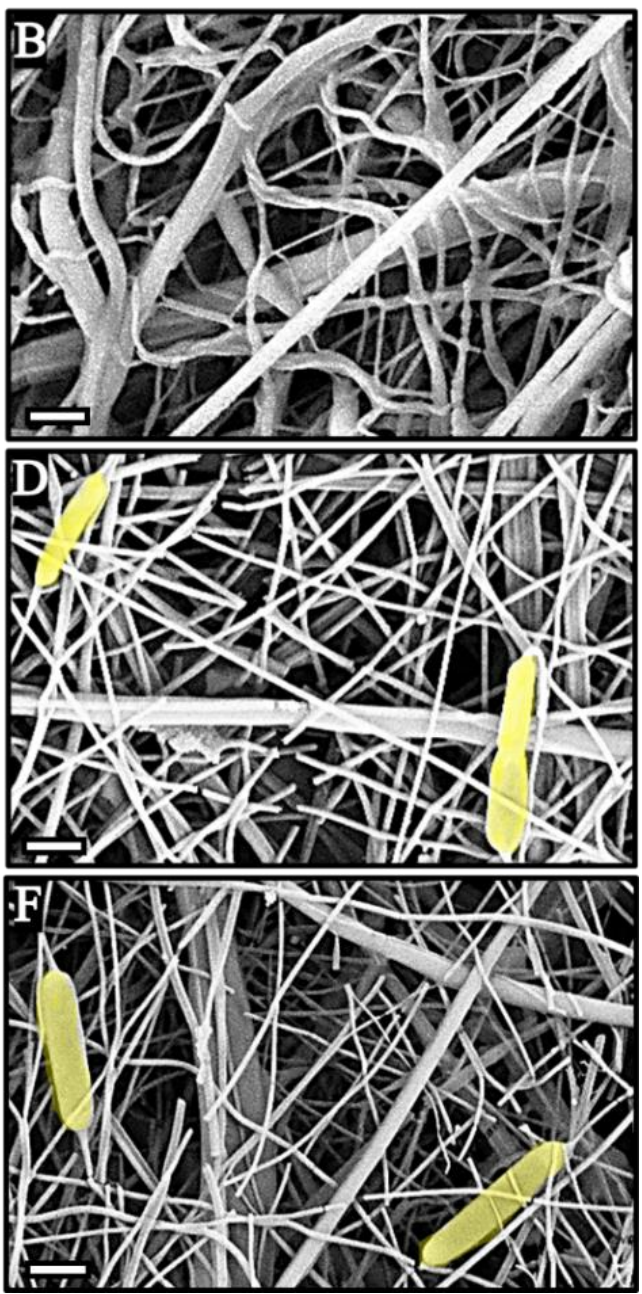

Figure 5.2. Probiotics are successfully incorporated within mesh PLGA:PEO (1:1) and PLGA:PEO (1:3) fiber architectures. SEM images of probiotic electrospun fibers composed of different ratios of PLGA and PEO. (A, B) blank fibers, (C, D) L. crispatus (L.C.)-containing fibers, and (E, F) L. acidophilus (L.a.)-containing fibers. Probiotic cells are highlighted and scale bars represent $2 \mu \mathrm{m}$. 
Layered Fiber

PLGA:PEO (1:1)
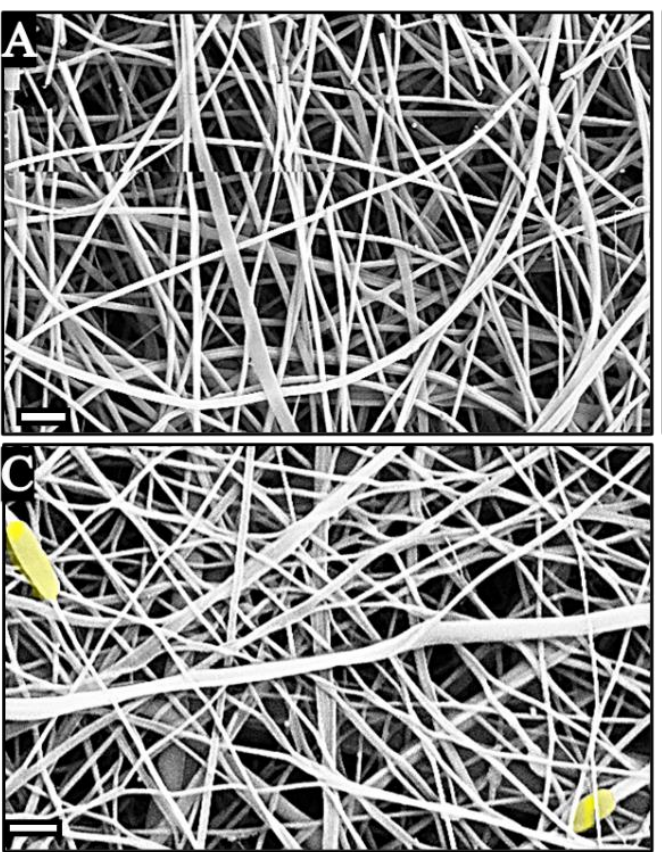

L.C.

L.a.

ENA ra

x)

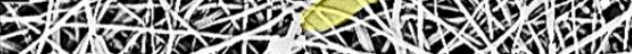

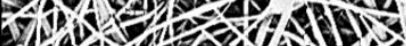

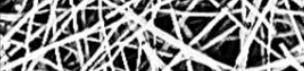

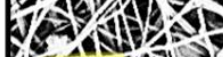

Layered Fiber

PLGA:PEO (1:3)
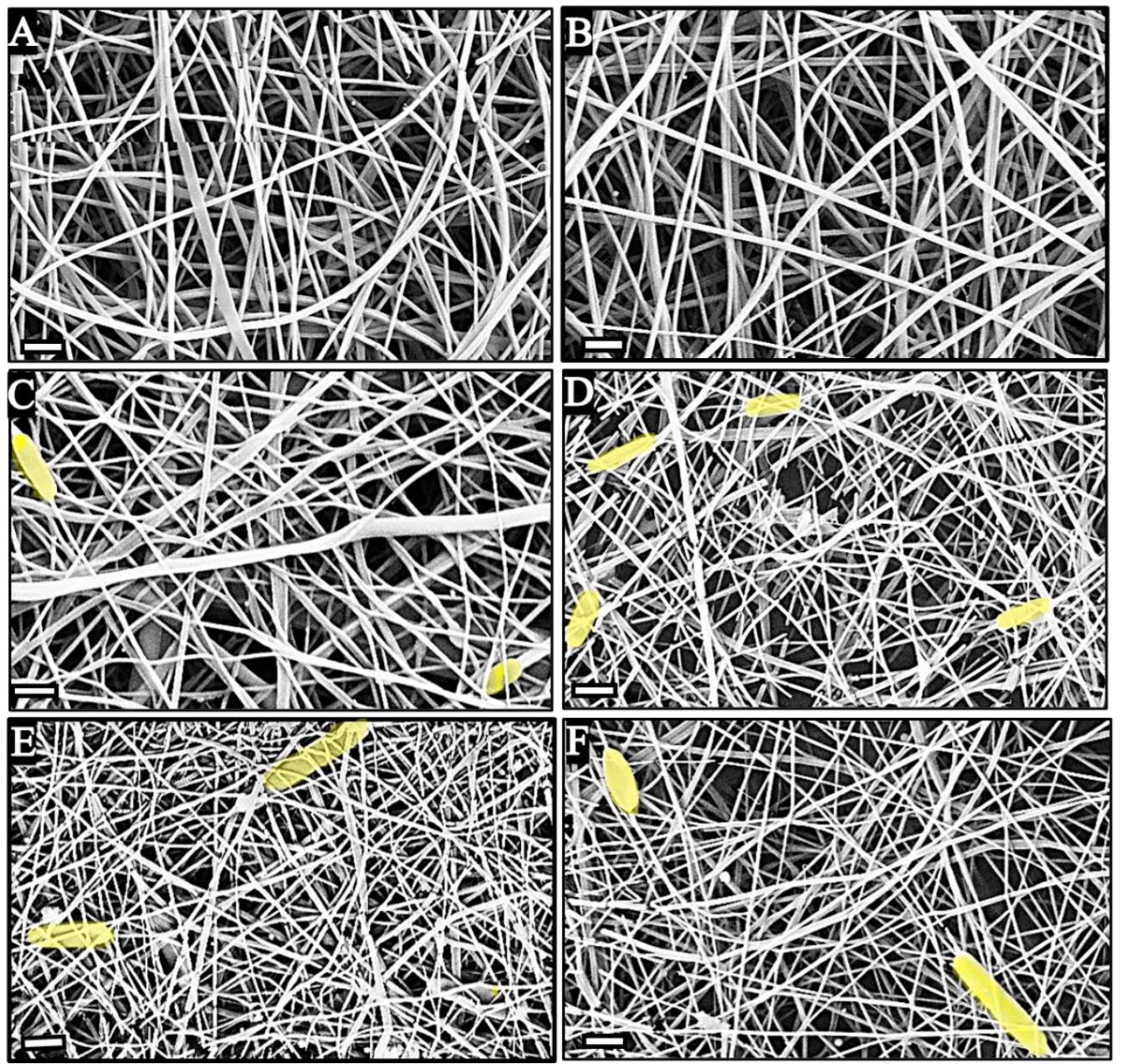

Figure 5.S2. Probiotics are successfully incorporated within layered PLGA:PEO

(1:1) and PLGA:PEO (1:3) fiber architectures. SEM images of probiotic electrospun fibers composed of different ratios of PLGA and PEO. (A, B) blank fibers, (C, D) L. crispatus (L.c.)-containing fibers, and (E, F) L. acidophilus (L.a.)containing fibers. Probiotic cells are highlighted and scale bars represent $2 \mu \mathrm{m}$. 
In addition to assessing the morphology of fibers in mesh and layered architectures, probiotic proliferation on the fiber surface was assessed by exposing the probiotic fibers to MRS broth for up to 2 wk. In both mesh and layered fibers, probiotic biofilms began to form on the fiber surfaces after $48 \mathrm{hr}$ and persisted through the 2 wk study (Figure 5.3 and Figure 5.S3). Both L. crispatus and L. acidophilus PLGA:PEO (1:1 and 1:3) fibers were able to localize probiotic growth on their surfaces, developing probiotic biofilms with similar morphologies. 

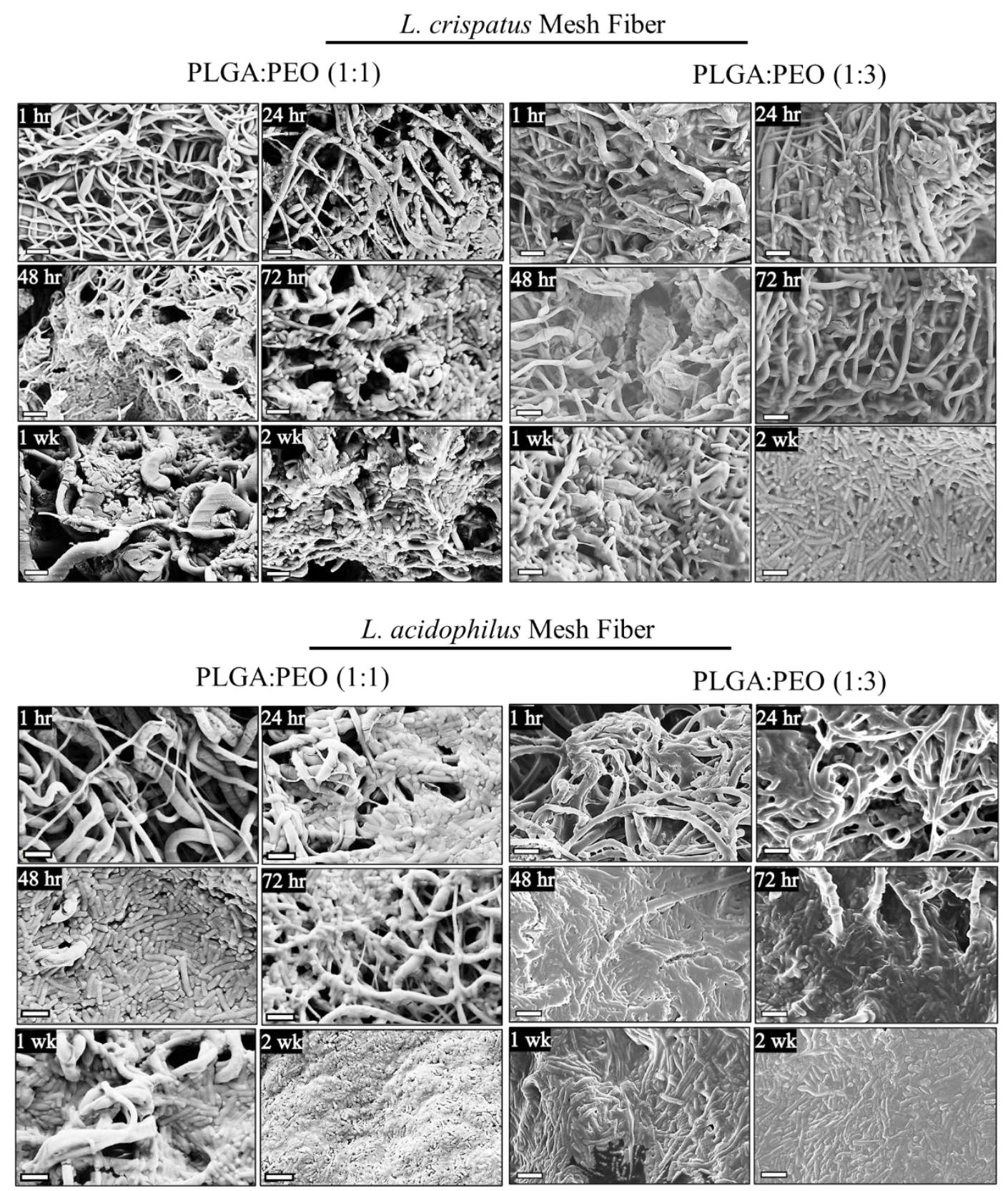

Figure 5.3. Probiotic biofilms are formed on mesh fiber surfaces after $48 \mathrm{hr}$ and persist through 2 wk. SEM images of mesh fibers composed of either PLGA:PEO (1:1) or (1:3) that incorporate L. crispatus or L. acidophilus after 1, 24, 48, and 72 $\mathrm{hr}$, and 1, and 2 wk in MRS broth. Scale bars represent $5 \mu \mathrm{m}$. 

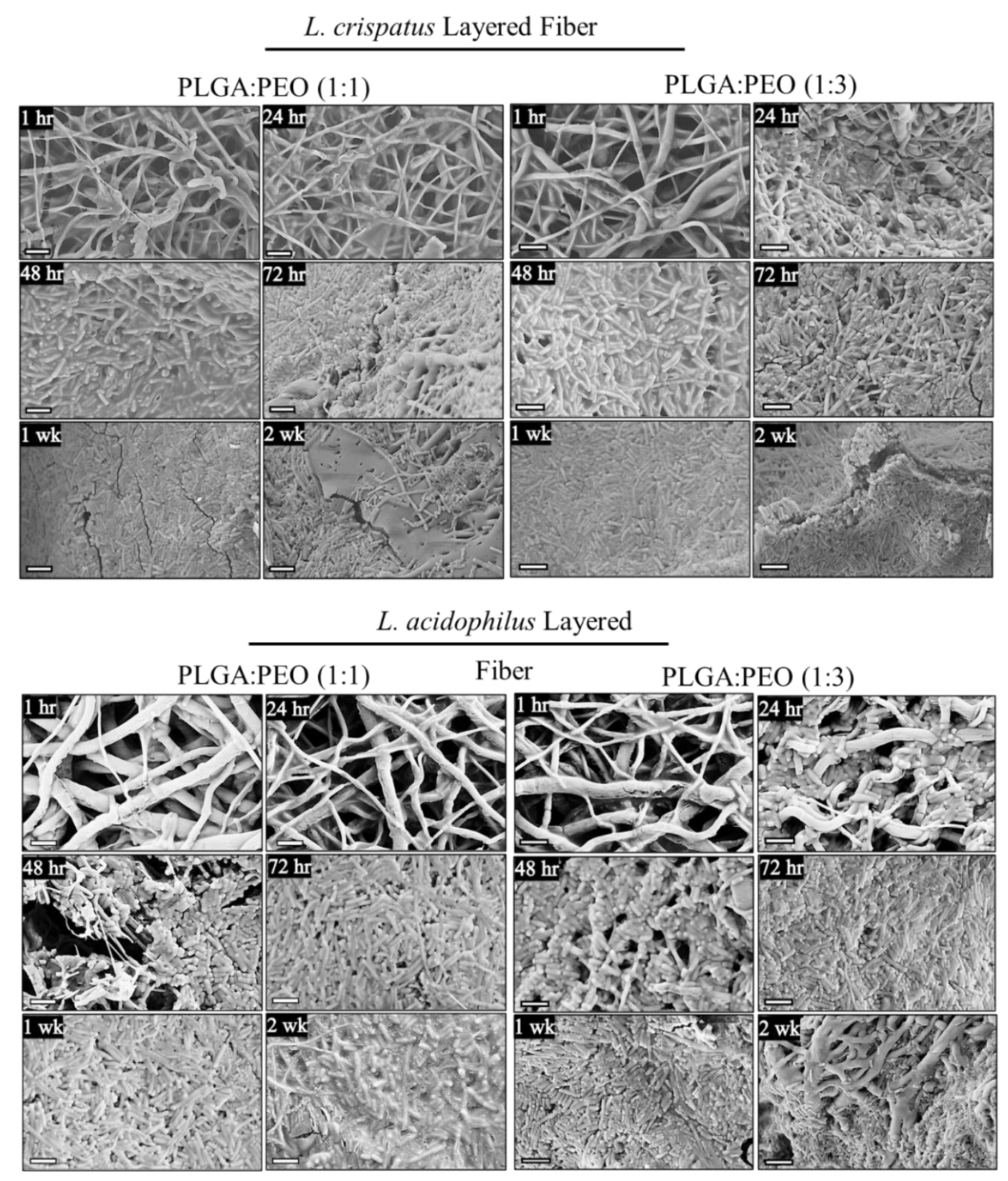

Figure 5S.3. Probiotic biofilms are formed on layered fiber surfaces after $48 \mathrm{hr}$ and persist through 2 wk. SEM images of layered fibers composed of either PLGA:PEO (1:1) or (1:3) that incorporate L. crispatus or L. acidophilus after $1,24,48$, and 72 $\mathrm{hr}$, and 1, and 2 wk in MRS broth. Scale bars represent $5 \mu \mathrm{m}$. 


\section{Fiber Degradation}

Micro and macroscale alterations to blank mesh and layered PLGA:PEO (1:1 and 1:3) fibers were evaluated for up to 2 wk (Figures 5.4 and 5.5A). In the mesh architecture, intact PEO fibers were observed for up to $48 \mathrm{hr}$, which was attributed to the simultaneous integration of soluble PEO with insoluble PLGA fibers during the dual-electrospinning process (Figure 5.4). Similarly, PEO fibers were still observed in layered PLGA:PEO (1:3) for up to $48 \mathrm{hr}$, while layered fibers with a smaller volume fraction of PEO (1:1) exhibited more rapid dissolution of PEO within $1 \mathrm{hr}$. Furthermore, both mesh and layered architectures displayed minor surface irregularities after 1 wk (arrows point to surface defects). From a microstructural perspective and despite the observation of slight surface irregularities, no significant changes in mesh or layered PLGA fiber diameters were observed after 2 wk $(2.23 \pm 0.39$ and $1.53 \pm 0.39 \mu \mathrm{m}$, respectively).

In line with these observations, the macrostructure of blank mesh and layered PLGA:PEO (1:1 and 1:3) fibers became more brittle after $72 \mathrm{hr}$ due to the

dissolution of PEO fibers from both formulation types (Figure 5.5). After 3 wk, mesh and layered PLGA:PEO (1:1) fibers had major ruptures and conglutination, while mesh and layered PLGA:PEO (1:3) fibers were completely dissolved. Macrostructural images of mesh PLGA:PEO (1:1) fibers are shown as representative for all formulations in Figure 5.5A.

In addition to changes in fiber morphology, the overall mass loss of blank mesh and layered PLGA:PEO (1:1 and 1:3) fibers was assessed for up to 2 wk 
(Figure 5.5B). For mesh PLGA:PEO (1:1 and 1:3) fibers, a 48 and $66 \%$ reduction in mass was observed after $1 \mathrm{hr}$, with total mass loss increasing to 53 and $75 \%$ after $48 \mathrm{hr}$, respectively. These values were in agreement with the general time frame observed for PEO dissolution. In comparison, layered PLGA:PEO (1:1 and $1: 3$ ) fibers had 46 and $68 \%$ mass loss after $1 \mathrm{hr}$ and 46 and $80 \%$ total mass loss after $48 \mathrm{hr}$. After $48 \mathrm{hr}$, all fibers had negligible mass loss for the remainder of 2 wk. 
Blank Mesh Fiber

PLGA:PEO (1:1)
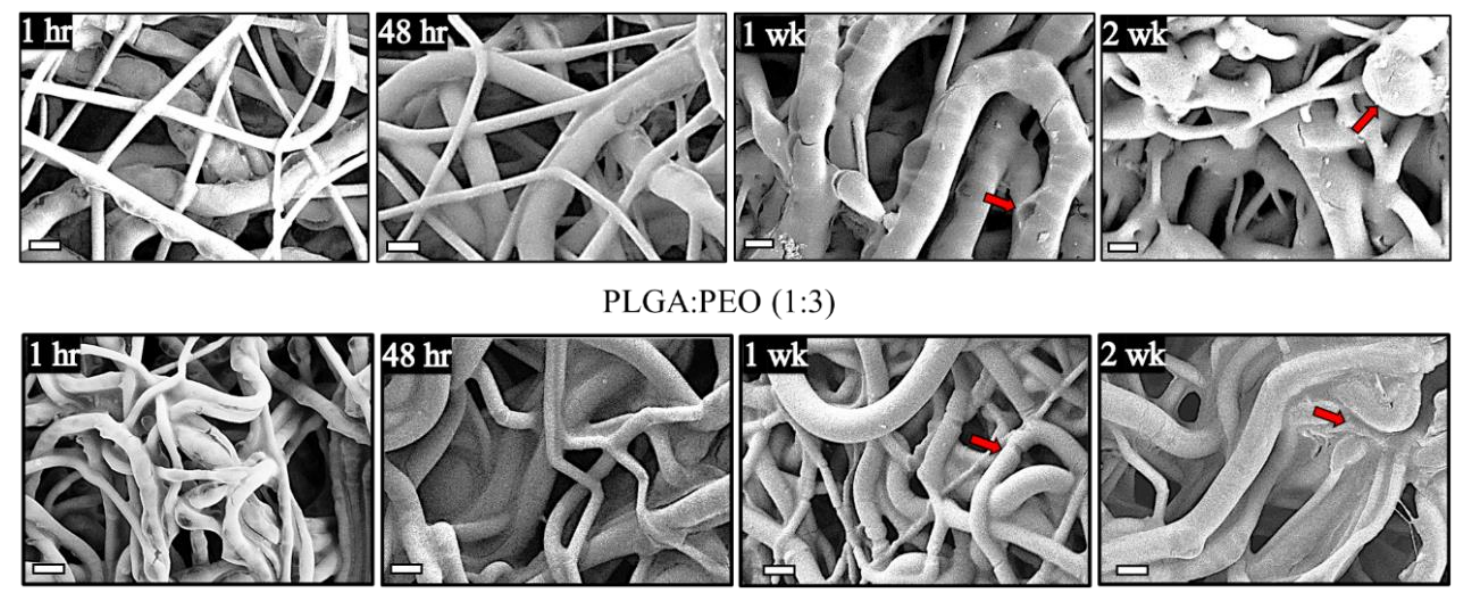

Blank Layered Fiber

PLGA:PEO (1:1)
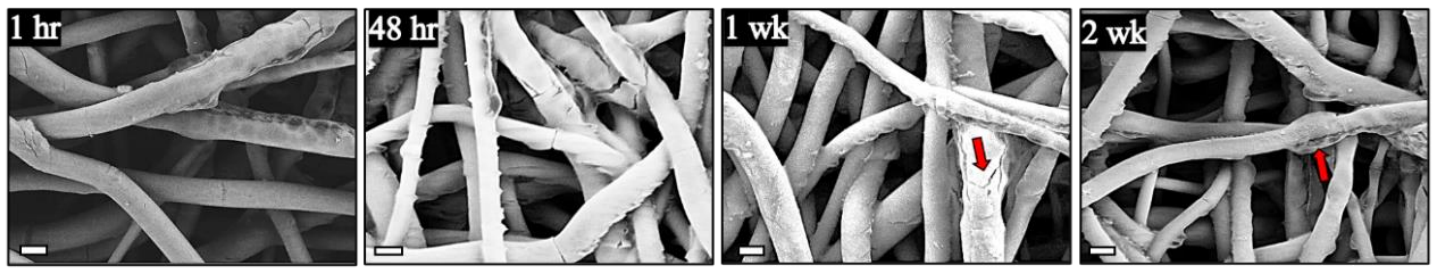

PLGA:PEO (1:3)
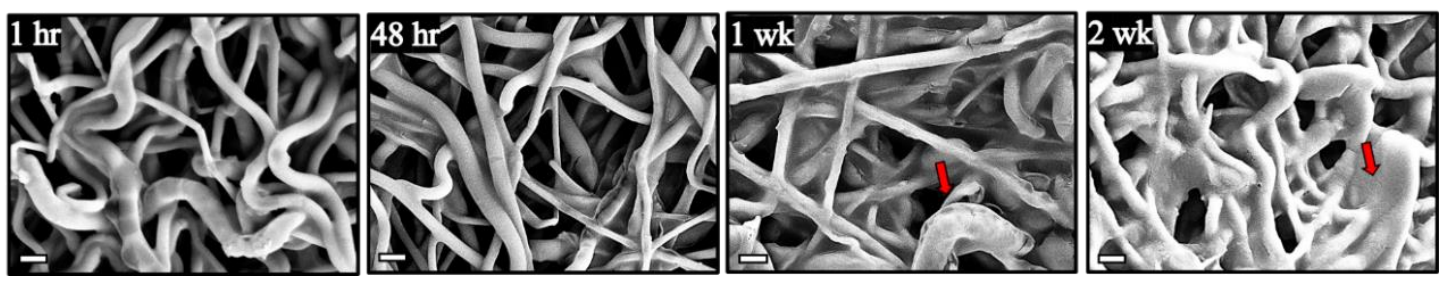

Figure 5.4. SEM images of mesh and layered blank fibers after $1 \mathrm{hr}, 48 \mathrm{hr}, 1 \mathrm{wk}$, and 2 wk degradation time points. While the PEO portion of mesh fibers (1:1 and 1:3) dissolves after $48 \mathrm{hr}$, PEO fibers in the layered (1:1) and (1:3) architecture dissolve after 1 and $48 \mathrm{hr}$, respectively. For both architectures, PLGA maintains its overall fiber structure for up to 2 wk. Arrows show surface roughening of the PLGA and scale bars represent $2 \mu \mathrm{m}$. 

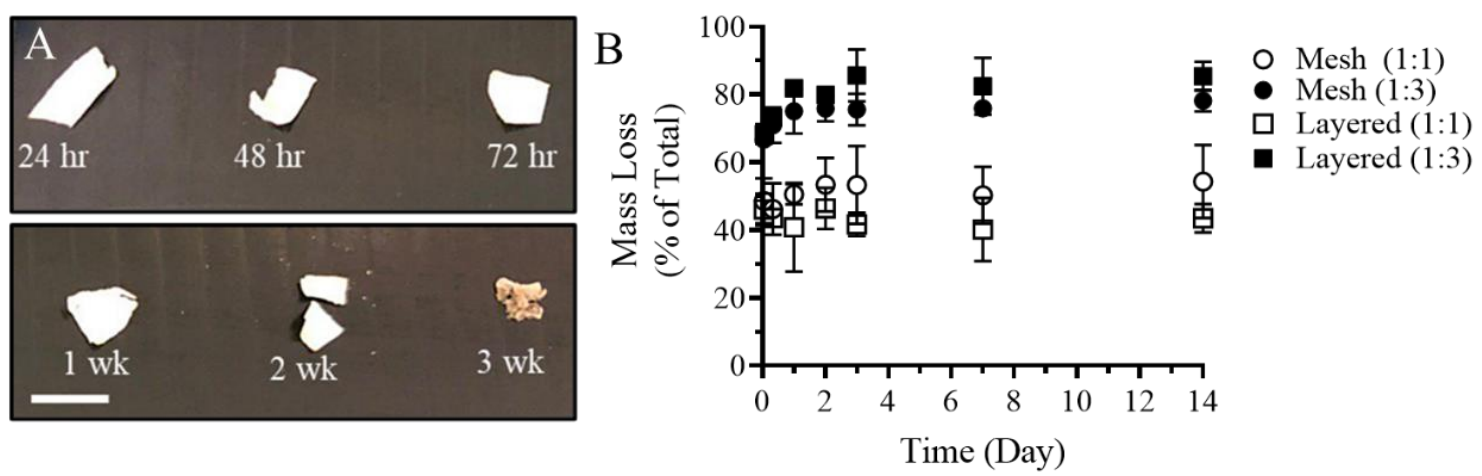

Figure 5.5. (A) Macrostructural images of blank mesh PLGA:PEO (1:1) fibers over the duration of the 3 wk degradation study. Similar macrostructural degradation was observed within mesh (1:3) and layered (1:1 and 1:3) fibers. (B) Percent of total mass loss, relative to starting mass, of blank mesh and layered fibers electrospun with different flow rate ratios (1:1 and 1:3). Mass loss values are shown as the mean \pm standard deviation of three independent fiber batches. Scale bar represents $1 \mathrm{~cm}$.

In line with these observations, the macrostructure of blank mesh and layered PLGA:PEO (1:1 and 1:3) fibers became more brittle after $72 \mathrm{hr}$ due to the dissolution of PEO fibers from both formulation types (Figure 5.4). After 3 wk, mesh and layered PLGA:PEO (1:1) fibers had major ruptures and conglutination, while mesh and layered PLGA:PEO (1:3) fibers were completely dissolved. Macrostructural images of mesh PLGA:PEO (1:1) fibers are shown as representative for all formulations in Figure 5.4A.

In addition to changes in fiber morphology, the overall mass loss of blank mesh and layered PLGA:PEO (1:1 and 1:3) fibers was assessed for up to 2 wk 
(Figure 5.4B). For mesh PLGA:PEO (1:1 and 1:3) fibers, a 48 and $66 \%$ reduction in mass was observed after $1 \mathrm{hr}$, with total mass loss increasing to 53 and $75 \%$ after $48 \mathrm{hr}$, respectively. These values were in agreement with the general time frame observed for PEO dissolution. In comparison, layered PLGA:PEO (1:1 and 1:3) fibers had 46 and $68 \%$ mass loss after $1 \mathrm{hr}$ and 46 and $80 \%$ total mass loss after $48 \mathrm{hr}$. After $48 \mathrm{hr}$, all fibers had negligible mass loss for the remainder of 2 wk.

\section{Probiotic Release and Proliferation}

The cumulative release and proliferation of probiotics from mesh and layered PLGA:PEO (1:1 and 1:3) fibers were evaluated over 2 wk (Figure 5.6). The total probiotic concentration, accounting for probiotic release from, and proliferation within all mesh (Figure 5.6A) and layered (Figure 6B) fibers reached $10^{8} \mathrm{CFU} / \mathrm{mg}$ fiber after $48 \mathrm{hr}$ and $10^{9} \mathrm{CFU} / \mathrm{mg}$ after 6 days. After $2 \mathrm{wk}$, the total release and proliferation observed from different mesh fiber formulations ranged from $3.8 \times 10^{9}$ and $6.6 \times 10^{9} \mathrm{CFU} / \mathrm{mg}$, and was similar to that observed from layered fibers $\left(2.4 \times 10^{9}\right.$ to $\left.6.1 \times 10^{9} \mathrm{CFU} / \mathrm{mg}\right)$. Across mesh and layered architectures with the same Lactobacillus type or polymer composition, similar levels of probiotic release and proliferation were observed after 2 wk $(P>0.05)$. 

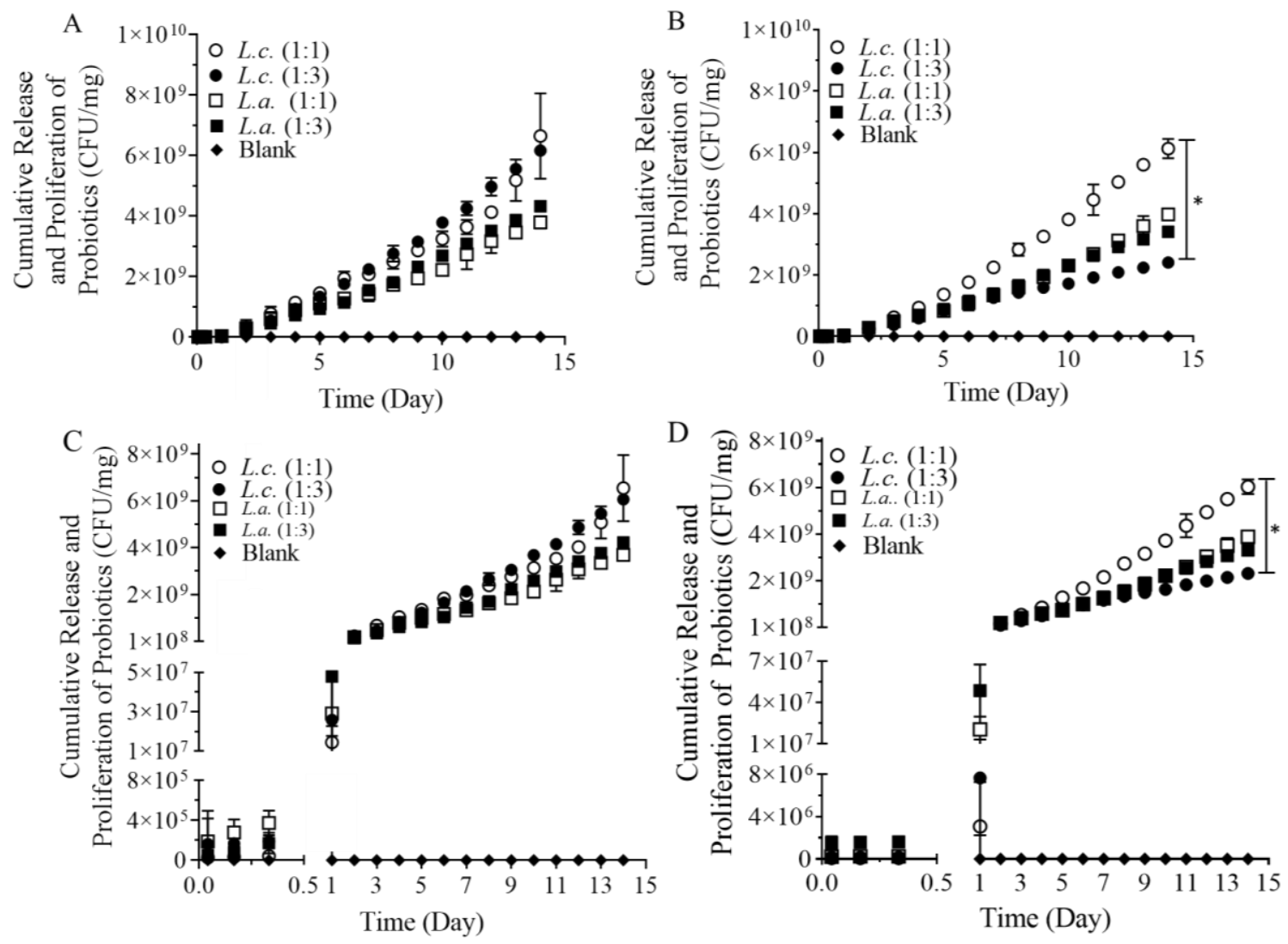

Figure 5.6. The cumulative release and proliferation of L. crispatus (L.C.) and $L$. acidophilus (L.a.) from ( $\mathrm{A}, \mathrm{C})$ mesh and $(\mathrm{B}, \mathrm{D})$ layered fibers over 2 wk. Panels $\mathrm{C}$ and $D$ highlight different concentration ranges of release from panels $A$ and $B$ based on the time frame of release. All mesh and layered fiber formulations demonstrate high levels of cumulative probiotic release and proliferation, reaching $10^{8}$ and $10^{9} \mathrm{CFU} / \mathrm{mg}$ fiber by $48 \mathrm{hr}$ and 6 days, respectively. Total release and proliferation after 2 wk were similar between groups within each of the mesh and layered architectures, as a function of the area under the curve and total release and proliferation $(P>0.05)$. The only exception was observed for layered fiber architectures, in which L.C. PLGA:PEO (1:1) released significantly more L.c. than L.C. PLGA:PEO (1:3) fibers, based on total release and area under the curve 
measurements after 2 wk $(P \leq 0.05)$. Across mesh and layered architectures with the same Lactobacillus type or polymer composition, similar levels of probiotic release were observed after 2 wk $(P>0.05)$. Release values are shown as the mean \pm standard deviation of three independent fiber batches. Statistical significance between experimental groups, as calculated by one-way ANOVA, is represented by ${ }^{*} \mathrm{P} \leq 0.05$.

For both mesh and layered architectures, no statistical significance was observed in total probiotic release and proliferation within mesh or layered fiber groups, as a function of Lactobacillus type $(P>0.05)$, as assessed by comparing both area under the curve and total cumulative release and proliferation after 2 wk. Similarly, as a function of PLGA:PEO composition (flow rate), fibers within mesh or layered fiber groups that were electrospun with different PLGA:PEO flow rates, displayed similar amounts of release after $2 \mathrm{wk}$, regardless of Lactobacillus type. The one exception was that layered $L$. crispatus PLGA:PEO (1:1) fibers had significantly higher probiotic release relative to layered L. crispatus PLGA:PEO (1:3) fibers after 2 wk $(P \leq 0.05)$. Overall, mesh and layered L. crispatus PLGA:PEO fibers, electrospun with equal flow rates (1:1 composition), demonstrated higher, albeit non-significant amounts of probiotic release, relative to all other formulations, with $2.1 \times 10^{9}$ and $2.3 \times 10^{9} \mathrm{CFU} / \mathrm{mg}$ released after $1 \mathrm{wk}$, and $6.6 \times 10^{9}$ and $6.1 \times 10^{9} \mathrm{CFU} / \mathrm{mg}$ after $2 \mathrm{wk}$.

The mesh and layered probiotic release and proliferation curves followed a zero-order release trend $\left(R^{2}=0.93\right.$ to 0.99$)$ after $48 \mathrm{hr}$, indicating a linear release of probiotics with respect to time. While no statistical significance was observed 
within or across architecture types, the highest and lowest rates of release within mesh fibers were observed from L. crispatus PLGA:PEO (1:1) and L. acidophilus PLGA:PEO (1:3) formulations, with slopes of $4.1 \times 10^{8}$ and $2.7 \times 10^{8}$ $\mathrm{CFU} /(\mathrm{mg} \cdot$ day $)$, respectively $(P>0.05)$. Within layered architectures, the highest and lowest release/proliferation rates were obtained from L. crispatus PLGA:PEO (1:1) and L. crispatus PLGA:PEO (1:3) fibers, with corresponding slopes of $4.6 \mathrm{x}$ $10^{8}$ and $1.8 \times 10^{8} \mathrm{CFU} /(\mathrm{mg} \cdot$ day $)(\mathrm{P}>0.05)$.

\section{Lactic Acid Production and pH Change}

Lactic acid production and $\mathrm{pH}$ changes were assessed from both blank mesh/layered fibers and probiotic-loaded mesh/layered fibers to compare with probiotic release and proliferation. The cumulative amounts of D- and L-lactic acid produced from mesh and layered fibers are shown in Figure 5.7 and correlate with the high levels of probiotic release and proliferation observed on the fiber surface. Mesh and layered fibers incorporating L. crispatus and L. acidophilus produced as much as 83 and $59 \mathrm{mg}$ of $\mathrm{D}$-lactic acid/mg fiber, respectively, over $2 \mathrm{wk}$, relative to blank fibers, which produced no lactic acid. Despite a slightly higher trend observed for D-, relative to L-lactic acid production for each mesh and layered formulation, similar cumulative amounts of D- and L-lactic acid were produced for each over the 2 wk duration $(P>0.05)$. Similarly, mesh as compared to layered fibers, that were electrospun with the same Lactobacillus type and flow rate (polymer composition), produced similar amounts of $D$ - and L-lactic acid $(P>0.05)$. 
Within mesh (Figure 5.7A-D) and layered (Figure 5.7E-H) fiber architectures, similar D and L-lactic acid production was observed as a function of Lactobacillus type or composition. Among mesh fiber formulations, the highest concentrations of D- and L-lactic acid were both produced from L. crispatus PLGA:PEO 1:3 fibers (D: 83.32 and L: $63.03 \mathrm{mg} / \mathrm{mg}$ fiber, Figure 5.7B) over 2 wk, while the highest amounts were produced from layered L. crispatus PLGA:PEO 1:1 (D: 53.75 mg/mg fiber, Figure 5.7E) and L. acidophilus PLGA:PEO 1:3 (L: $39.67 \mathrm{mg} / \mathrm{mg}$ fiber, Figure $\mathbf{5 . 7 H}$ ) formulations. 
Mesh Fiber
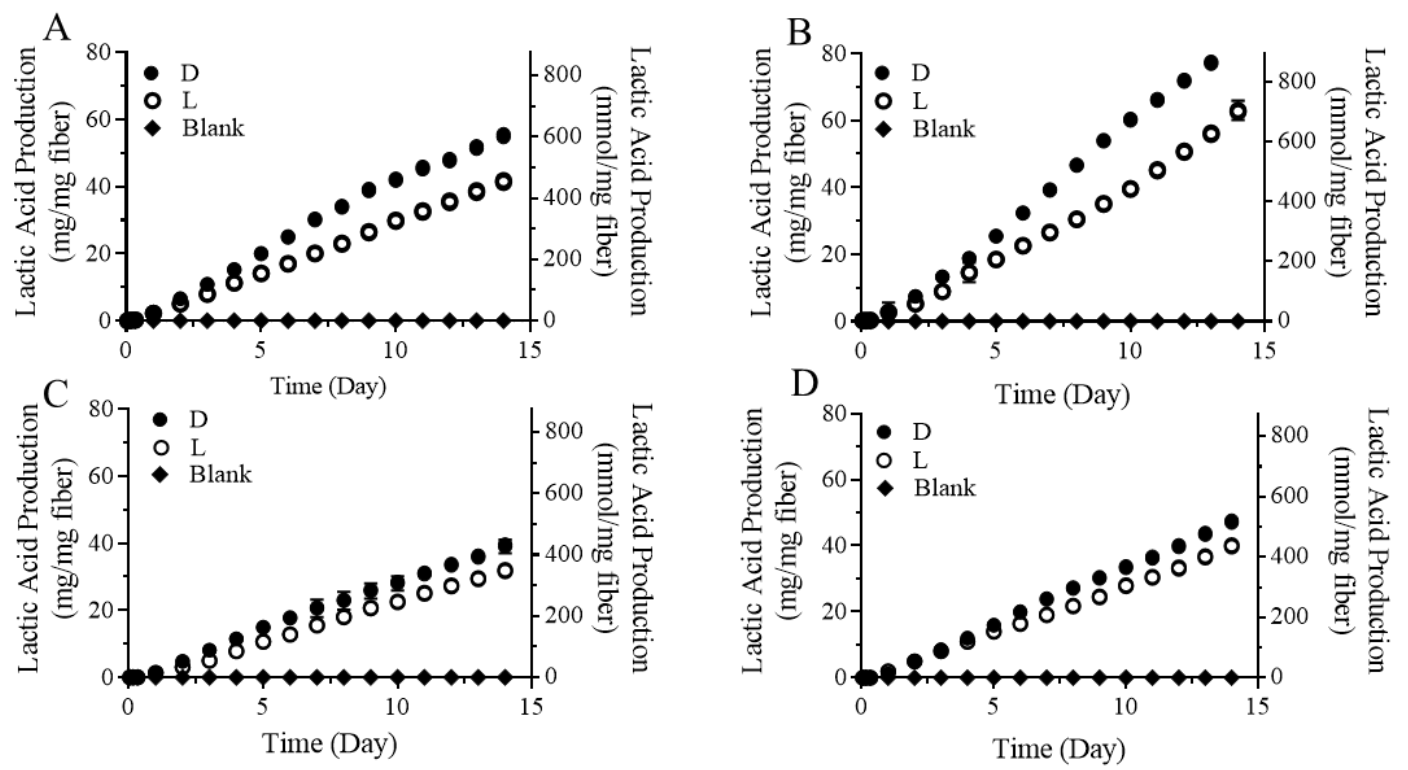

Layered Fiber
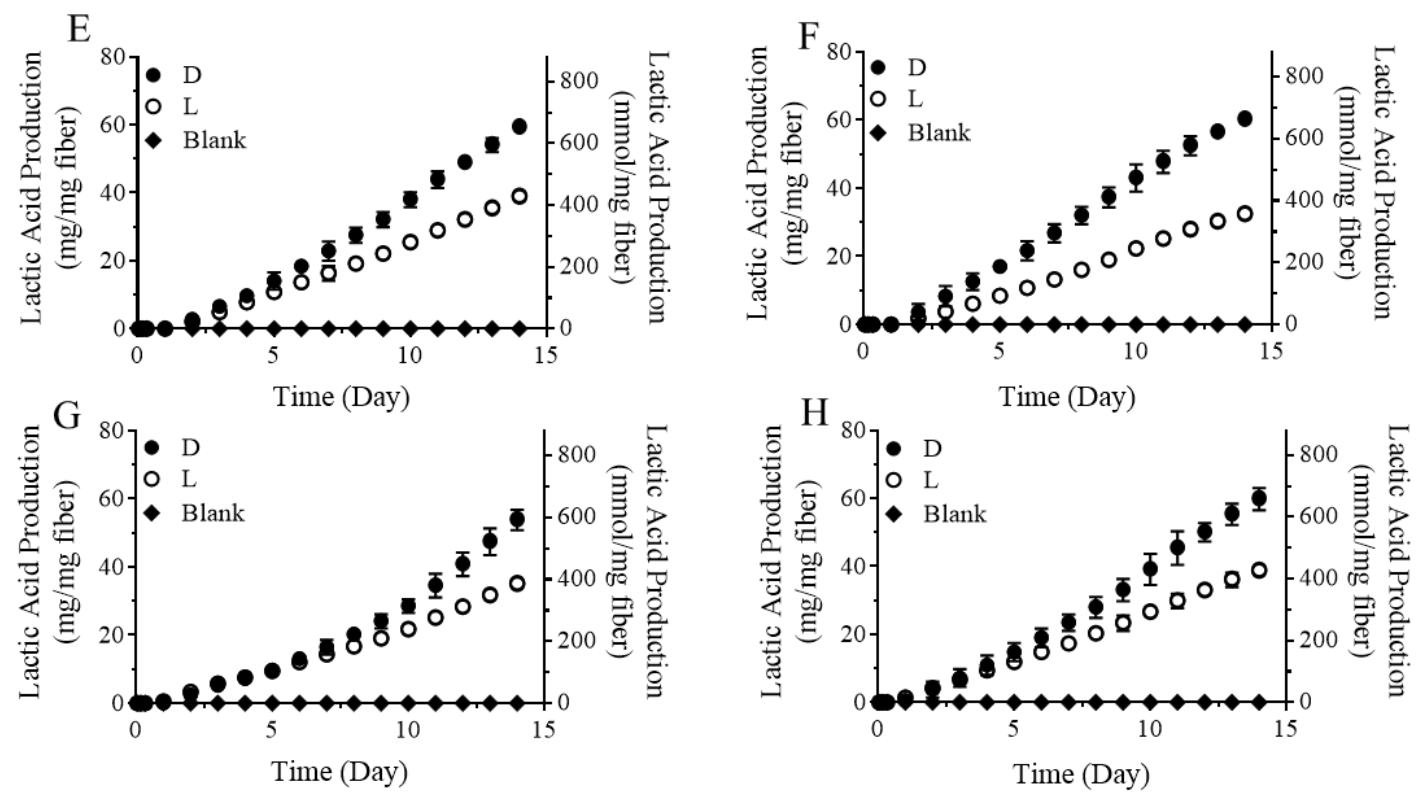

Figure 5.7. Lactic acid production from mesh $(A, B, C$, and $D)$ and layered $(E, F$, $\mathrm{G}$, and $\mathrm{H}$ ) fibers. (A, E) L. crispatus PLGA:PEO (1:1); (B, F) L. crispatus PLGA:PEO (1:3): (C, G) L. acidophilus PLGA:PEO (1:1); and (D, H) L. acidophilus PLGA:PEO (1:3) fibers. Mesh and layered fibers incorporating $L$. crispatus and $L$. acidophilus produced as much as 83 and $59 \mathrm{mg}$ D-lactic acid/mg fiber respectively 
over 2 wk, relative to blank fibers that produced negligible levels of D- and L-lactic acid (below assay detection limit of $\sim 1.5 \mu \mathrm{g} / \mathrm{mL}$ ). Similar lactic acid production was observed between mesh and layered fibers electrospun with the same Lactobacillus type or fiber composition, as calculated by both area under the curve and total lactic acid production $(P>0.05)$. Within mesh and layered architectures with the same probiotic type or fiber composition, no differences were observed in D- and L-lactic acid production ( $P>0.05)$. Lactic acid production is shown as the mean \pm standard deviation of three independent fiber batches. In some cases, error bars appear smaller than the symbol size. Statistical significance between experimental groups was determined using one-way ANOVA.

In addition to evaluating the amount of lactic acid production, the rate of lactic acid produced per day was evaluated, demonstrating a linear trend for all mesh and layered fiber formulations $\left(R^{2}=0.93\right.$ to 0.99$)$. Overall, similar $D$ - and Llactic acid production rates (slopes) were observed between mesh and layered architecture fibers electrospun with the same Lactobacillus type or composition (flow rate) $(P>0.05)$. Within mesh formulations, similar rates of $D$ - or L-lactic acid production were observed as a function of Lactobacillus type. Similarly in mesh fibers, as a function of polymer composition (1:1 or 1:3), L. acidophilus formulations had similar rates of D-lactic acid production, while L. crispatus PLGA:PEO (1:3) had a significantly higher rate of L-lactic acid production relative to L. crispatus PLGA:PEO (1:1) $(P \leq 0.05)$. The production rates of $D$ - and L-lactic acid were similar across different formulations of layered fibers with either different Lactobacillus type or polymer composition $(P>0.05)$. The highest rates of $D$-lactic 
acid production were observed from mesh and layered L. crispatus PLGA:PEO (1:3) fibers (6.22 and $4.32 \mathrm{mg} / \mathrm{mg}$ fiber-day, respectively), while the highest rate of L-lactic acid production was observed from mesh L. crispatus PLGA:PEO (1:3, $4.52 \mathrm{mg} / \mathrm{mg}$ fiber-day) and layered $L$. acidophilus PLGA:PEO (1:1, $3.43 \mathrm{mg} / \mathrm{mg}$ fiber-day) fibers.

Corresponding with lactic acid production, a significant decrease in the eluate $\mathrm{pH}$ was observed for mesh (Figure 5.8A, B) and layered (Figure 5.8C, D) PLGA:PEO (1:1 and 1:3) fibers. A decrease from an initial pH of 6 to 4 or 4.5 was observed with 24 to $48 \mathrm{hr}$, while a subsequent decrease to 3.0 or 3.5 was observed after $1 \mathrm{wk}(\mathrm{P} \leq 0.0001)$. These changes were observed regardless of probiotic type or polymer composition (1:1 or 1:3) (Figure 5.8). In contrast, no changes in $\mathrm{pH}$ were observed for blank PLGA:PEO (1:1 and 1:3) mesh and layered fibers, relative to probiotic-containing fiber groups. 

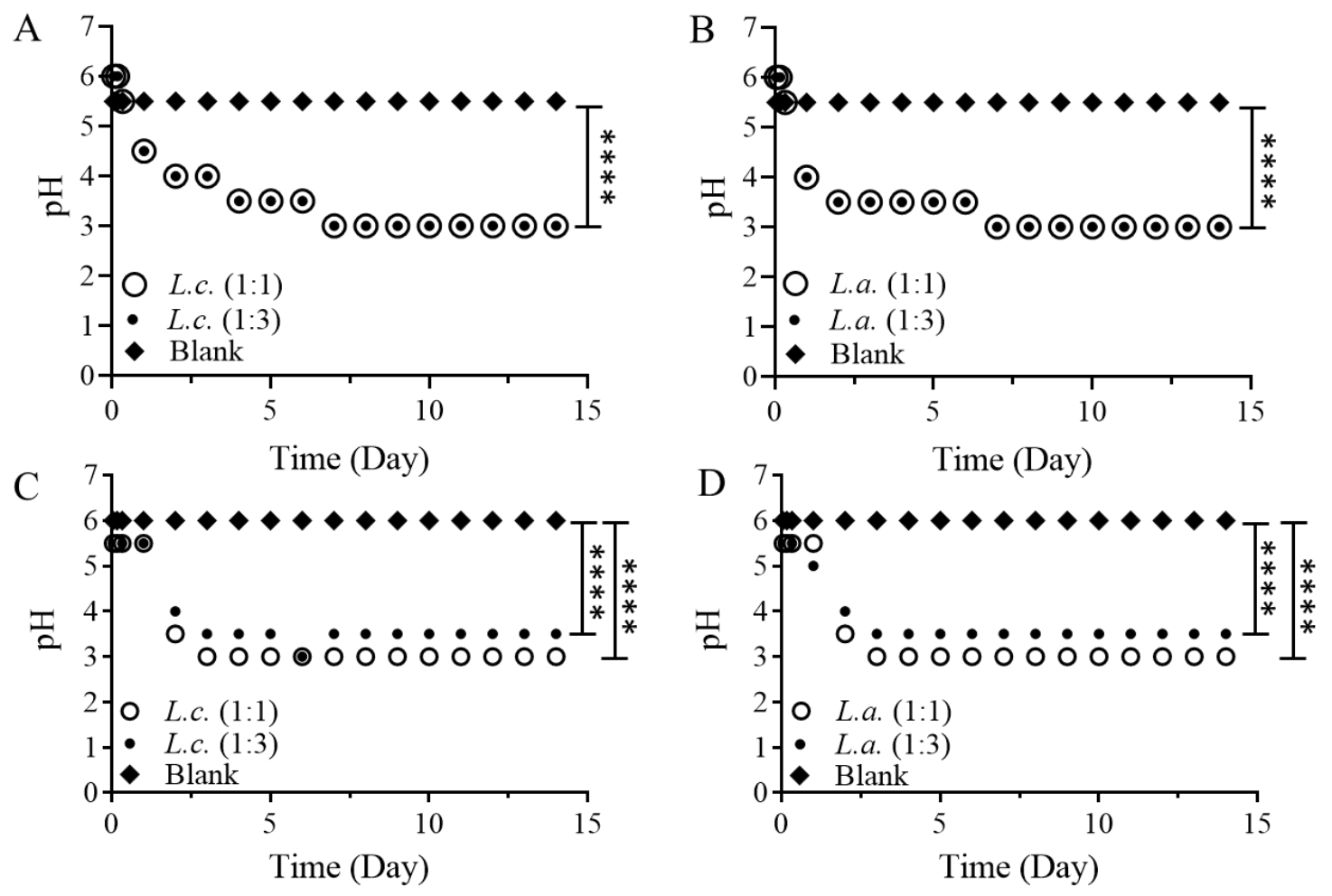

Figure 5.8. The $\mathrm{pH}$ of PLGA:PEO mesh and layered fiber eluates was measured over 2 wk. Mesh (A) L. crispatus (L.C.) and (B) L. acidophilus (L.a.) and layered (C) L.c. and (D) L.a. fibers electrospun with different PLGA:PEO (1:1 and 1:3) flow rate ratios. The lactic acid produced by probiotics caused a significant decrease in the $\mathrm{pH}$ of all fiber eluates within the first week $(\mathrm{P} \leq 0.0001)$ that persisted, regardless of daily media changes, over 2 wk. No changes in $\mathrm{pH}$ were observed in blank fiber eluates $(\mathrm{P}>0.05) . \mathrm{pH}$ values are shown as the mean \pm standard deviation of eluates from three independent fiber batches and error bars are smaller than the symbol size. Statistical significance between experimental groups, as calculated by one-way ANOVA, is represented by ${ }^{* * * *} \mathrm{P} \leq 0.0001$. 


\section{In Vitro Efficacy of Mesh and Layered Fibers}

To evaluate the ability of probiotic fibers to inhibit $G$. vaginalis, the fiber formulation with the highest probiotic release/proliferation and fabrication yield was selected for co-culture with $G$. vaginalis. Here, PLGA:PEO (1:1) mesh or layered fibers were co-cultured with $G$. vaginalis at equivalent concentrations $\left(10^{6}\right.$ $\mathrm{CFU} / \mathrm{mL}$ ) for up to $48 \mathrm{hr}$ under anaerobic conditions at $37^{\circ} \mathrm{C}$. An equivalent dose of mesh or layered L. crispatus PLGA:PEO (1:1) fibers $\left(1.2 \mathrm{mg} / \mathrm{mL}, 10^{6} \mathrm{CFU} / \mathrm{mL}\right.$, based on L. crispatus actual loading in PEO fibers) was administered with $G$. vaginalis $\left(10^{6} \mathrm{CFU} / \mathrm{mL}\right)$. Figure 5.9 shows the species viability as measured by CFU counts for free L. crispatus or free G. vaginalis; mesh or layered L. crispatus PLGA:PEO (1:1) fibers alone; or free L. crispatus or mesh/layered L. crispatus PLGA:PEO (1:1) fibers co-cultured with G. vaginalis for 4, 24, or $48 \mathrm{hr}$. For $L$. crispatus mesh PLGA:PEO (1:1) fibers, a 2.2- ( $P>0.05)$, 8- $(P \leq 0.001)$, and 9.3$\log (P \leq 0.0001)$ reduction in $G$. vaginalis viability was observed after 4,24 , and 48 hr of co-culture, respectively. Similarly, L. crispatus layered PLGA:PEO (1:1) fibers completely inhibited G. vaginalis growth after 24 and $48 \mathrm{hr}$ (6.5- and 9.5-log reduction in viability, $P \leq 0.0001$ ), while no significant decrease in $G$. vaginalis viability was observed after $4 \mathrm{hr}(2.3-\log$ reduction, $\mathrm{P}>0.05)$. 


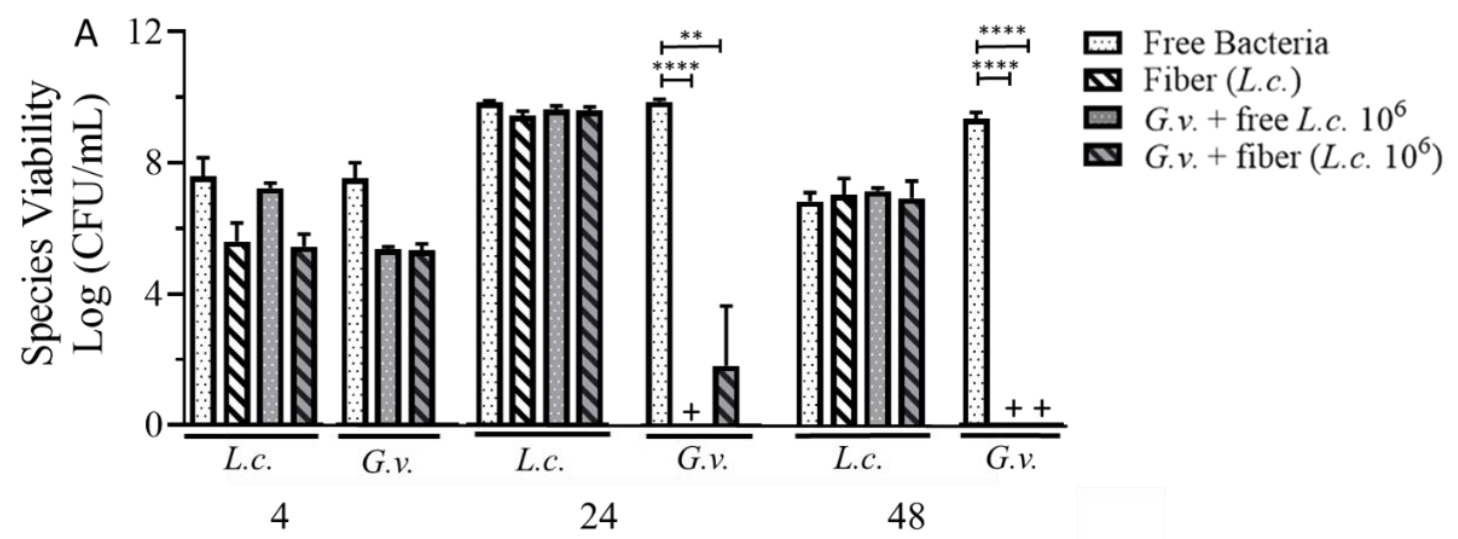

AdministrationTime (hr)

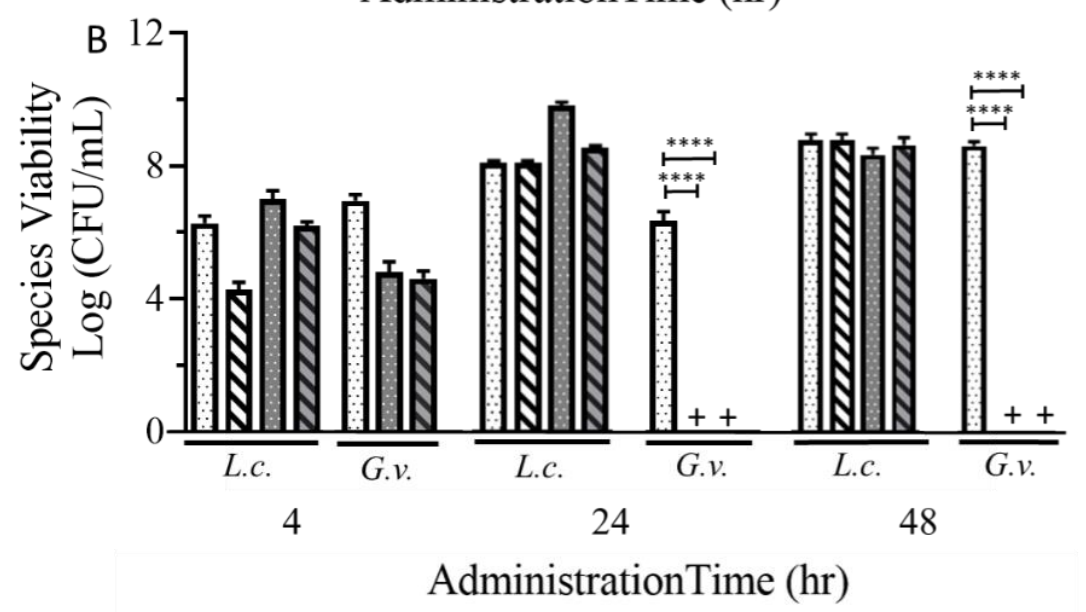

Figure 5.9. L. crispatus (L.c.) PLGA:PEO (1:1) mesh or layered fibers were cocultured with G. vaginalis (G.v.) at equal initial concentrations of $10^{6} \mathrm{CFU} / \mathrm{mL}$ for 4, 24, and 48 hr. (A) L. crispatus PLGA:PEO (1:1) mesh fibers significantly inhibited G. vaginalis viability after $24(P \leq 0.001)$ and $48 \mathrm{hr}(\mathrm{P} \leq 0.0001)$. Similarly, free $L$. crispatus inhibited $G$. vaginalis viability after both 24 and $48 \mathrm{hr}(\mathrm{P} \leq 0.0001)$. (B) L. crispatus PLGA:PEO (1:1) layered fibers significantly inhibited $G$. vaginalis viability after $24(P \leq 0.0001)$ and $48 \mathrm{hr}(P \leq 0.0001)$. Similarly, free L. crispatus inhibited G. vaginalis viability after both $24(P \leq 0.0001)$ and $48 \mathrm{hr}(\mathrm{P} \leq 0.0001)$. Columns with zero values are represented by "+", and the $\log (\mathrm{CFU} / \mathrm{mL})$ values are determined from the mean \pm standard deviation of eluates from three independent 
L. crispatus fiber or free L. crispatus batches. Statistical significance between experimental groups, as calculated by one-way ANOVA, is represented by ${ }^{* *} \mathrm{P} \leq$ 0.001 and ${ }^{* * * *} \mathrm{P} \leq 0.0001$.

\section{Discussion}

The administration of probiotics, specifically lactobacilli, can enhance female reproductive health by preventing or disrupting pathogenic bacteria adhesion and growth. Among different Lactobacillus species, L. crispatus and L. acidophilus, are two of the most abundant species in a healthy vaginal environment that have been used for BV treatment, due to strong epithelial cell adhesion and the production of high lactic acid concentrations ${ }^{418,419}$.

The intravaginal delivery of lactobacilli has shown promise to treat initial and recurrent $\mathrm{BV}$ infections, in particular, relative to oral delivery, due to the localized delivery of highly viable probiotic concentrations to the FRT. Different dosage forms including tablets ${ }^{337}$, capsules ${ }^{402}$, and suppositories $^{420}$ that incorporate probiotics have shown efficacy against BV infection. However, rapid probiotic release from these delivery platforms often results in transient effects, thereby requiring frequent administration, which can lead to low user adherence and recurrent infection. To address these challenges, there is a need to develop new delivery platforms focused on the maintenance and restoration of vaginal health, via localized and prolonged lactobacilli delivery. To date, only one platform, in the form of pod-based IVRs, has demonstrated sustained probiotic release in vitro on the order of $10^{7} \mathrm{CFU} /$ ring daily over 21 days. However, the prolonged residence of 
rings in the vaginal environment has been shown to result in bacterial matrices comprised of a wide variety of bacterial types and extracellular polysaccharides, potentially complicating the amelioration of infection ${ }^{407,408}$.

Recently, electrospun fibers have been applied in a variety of architectures to prolong the release of different active agents including antiretrovirals ${ }^{251,346,421,422}$ and antiviral proteins ${ }^{273}$ for intravaginal delivery. Furthermore, electrospun fibers may be a favorable dosage form by which to address challenges, such as low user adherence and emerging drug resistance, present with more frequently administered delivery platforms. Some of the benefits provided by electrospun fibers include the ability to integrate diverse polymer types to modulate release, the simplicity of the electrospinning process, and the flexibility to incorporate multiple types of active agents ${ }^{346}$. Recently, our group and others have extended the use of electrospinning to develop rapid-dissolve, "on-demand" intravaginal delivery platforms that incorporate probiotics with high viability and stability ${ }^{342,343,416}$. Given the long-standing need to develop sustained-release dosage forms that can maintain vaginal health and treat BV recurrence, here we sought to build upon rapid-dissolve fibers to create more complex architectures that integrate hydrophobic and hydrophilic polymers, to sustain probiotic release for BV treatment.

To first evaluate the feasibility of this approach, we assessed the incorporation of L. crispatus and L. acidophilus in mesh and layered fibers with different PLGA to PEO ratios. A previous study in our group showed that $L$. acidophilus, incorporated in PEO fibers spun in water at an initial concentration of 
$5 \times 10^{7} \mathrm{CFU} / \mathrm{mg}$ fiber, had a post-spun viability of $1.7 \times 10^{7} \mathrm{CFU} / \mathrm{mg}$ fiber ${ }^{416}$. In this study, a similar amount of L. crispatus or L. acidophilus was incorporated in the PEO portion of mesh and layered fibers, and we envisioned that probiotic incorporation in more complex mesh and layered fibers would enable the hydrophilic polymer to act as a probiotic reservoir, while the hydrophobic polymer would serve as a structural support to enhance probiotic proliferation and retention. Probiotic distribution in these fiber formulations was confirmed with SEM imaging, showing that probiotics were well-distributed within all fiber formulations (Figure

\section{2 and Figure 5.S2).}

To evaluate the in vitro degradation of blank mesh and layered fibers, fiber micro- and macrostructures were evaluated after exposure to SVF. Based on SEM images, mesh architecture PEO fibers generally remained intact for a longer duration (48 hr), relative to layered architecture PEO fibers $(1: 3,1 \mathrm{hr})$. These findings of more rapid PEO dissolution in the layered architectures may be attributed to the potential for increased entanglement between PEO and PLGA fibers in the interwoven mesh architecture, relative to discrete sections in the layered architecture. After $1 \mathrm{wk}$, the remaining PLGA fibers in both architectures had some surface roughening that remained through 2 wk. These fiber surface irregularities may be partially attributed to the relatively acidic environment $(\mathrm{pH} 4.5)$ of simulated vaginal fluid, which can accelerate fiber degradation. Other work, which studied the degradation of PLGA microspheres under different $\mathrm{pH}$ conditions, showed that an acidic environment augments polymer erosion by catalyzing the rupture of the ester linkage in the PLGA backbone ${ }^{423}$. Thus, while 
these acidic conditions may contribute to the surface roughening of PLGA fibers, these minor irregularities did not seem to substantially contribute to fiber degradation. After 2 wk, PLGA:PEO (1:3) fibers displayed a more melded and eroded appearance, relative to PLGA:PEO (1:1) fibers, which is likely attributed to the higher ratio of PEO to PLGA in the PLGA:PEO (1:3) formulations. While our study focused on dual- and layered electrospinning of PLGA and PEO fibers, studies that blended PLGA with PEO (as a porogen) have been shown to accelerate PLGA degradation by creating a more porous structure and increasing surface exposure to the surrounding eluate ${ }^{424}$.

In parallel with evaluating the micromorphology of fibers, the mass loss of different fiber architectures was measured after exposure to SVF. PLGA:PEO (1:1) mesh and layered fibers, comprised of final concentrations of 20 wt\% PEO and $\sim 80 \mathrm{wt} \%$ PLGA, had 48 and $46 \%$ weight loss after $1 \mathrm{hr}$, indicating the immediate nature of PEO dissolution as a primary contributor to mass loss. The same observation was made for PLGA:PEO (1:3) mesh and layered fibers containing $\sim 50 \mathrm{wt} \%$ PEO and $~ 50 \mathrm{wt} \%$ PLGA, which had higher mass losses (66 and 68\%) after $1 \mathrm{hr}$, stemming from the increased PEO content. There were subsequent minor decreases in total mass for both mesh and layered fibers with different compositions for up to $48 \mathrm{hr}$, followed by a plateau in weight loss. Overall, mesh and layered PLGA:PEO (1:1) fibers were shown to degrade more slowly, exhibiting major ruptures after 3 wk, while mesh and layered PLGA:PEO (1:3) fibers were completely dissolved within this same time frame. 
In order to evaluate the potential of mesh and layered fibers to extend delivery beyond that required for on-demand applications, probiotic release and proliferation were assessed from different architectures. For mesh and layered fibers with different Lactobacillus species and polymer compositions, cumulative probiotic release reached $\sim 10^{7} \mathrm{CFU} / \mathrm{mg}$ after $24 \mathrm{hr}$. After $48 \mathrm{hr}$, a linear trend in the cumulative release and proliferation of probiotic was observed, providing more than $10^{8} \mathrm{CFU} / \mathrm{mg}$ probiotic daily, which is on the order of or higher than the required daily dose of probiotic for clinical BV treatment $\left(10^{8} \mathrm{CFU} / \text { day }\right)^{425}$. For the mesh architectures, no statistical significance in probiotic release was observed as a function of probiotic type or polymer composition; however, in layered fibers, $L$. crispatus PLGA:PEO (1:1) released significantly higher $(P \leq 0.05)$ amounts of probiotic, relative to PLGA:PEO (1:3) fibers. In general, all tested formulations were able to release efficacious amount of probiotic for up to $2 \mathrm{wk}$, but it should be mentioned that mesh and layered PLGA:PEO (1:1) fibers better maintained their structures over the 2 wk study, while PLGA:PEO (1:3) were more fragile and susceptible to ruptures.

Relative to this study, to our knowledge, only one other platform has demonstrated sustained lactobacilli release. In this previous work, L. gasseri was incorporated in silicone IVRs and achieved release on the order of 1.1 to $14 \times 10^{7}$ CFU/ring each day for up to 21 days $^{340}$. While higher probiotic release was observed in our study, release was conducted in MRS ( $\mathrm{pH}$ 6.2), relative to PBS $(\mathrm{pH} 7.2)$ in the former IVR study. We acknowledge that release eluate may impact bacterial release and proliferation; however, in our hands, we observed minimal 
release in the relatively low glucose $(5 \mathrm{~g} / \mathrm{L})$ simulated vaginal fluid (SVF), relative to higher glucose-containing MRS (20 g/L), potentially indicating a lack of nutrients available to foster probiotic survival. In future studies, it will be important to assess the impact of different eluates to best represent the conditions relevant to intravaginal delivery and to correlate in vitro with in vivo probiotic release and proliferation.

Additionally, it is important to note that differences in fiber and IVR morphologies may play a role in release. In fibers, probiotics were incorporated within the PEO fiber portion, which rapidly degrades in solution, leaving probiotics to intermingle and reside in the surrounding PLGA fabric. In this regard, probiotics are "incorporated" but not actually "encapsulated," as they are in IVRs. Hence, IVRs may more specifically show release, whereas probiotics may desorb from the fiber surface or diffuse from the matrix. Furthermore, this suggests that probiotics are more exposed to the surrounding eluate in fibers, thus the choice of eluate may play a more significant role in observing their release and viability. In upcoming in vivo studies, we look forward to investigating how probiotic release or diffusion translates to in vivo viability and colonization. These results would help to validate appropriate in vitro conditions by which to test these new sustained-delivery probiotic platforms. For fibers and other delivery vehicles, it has previously been acknowledged, that in vitro and in vivo release often differ ${ }^{197}$ and as such, it is important to establish mimetics that accurately reflect in vivo conditions and features of the physiological environment for intravaginal (and other) applications. 
In addition to the ability of fibers to provide high levels of probiotic release, one of the observations with polymer-based delivery platforms, which in this case may benefit probiotic delivery and vaginal health, is the susceptibility to biofilm formation ${ }^{426}$. Previous work has shown that IVRs are susceptible to biofilm formation by a variety of bacteria, which, in some cases may limit their long-term use $^{407,408}$. In our studies, in addition to high levels of probiotic release, we observed probiotic proliferation on the fiber surfaces after $72 \mathrm{hr}$, which persisted through the 2 wk study (Figure 5.3 and Figure 5S.3). We anticipate that the presence of such high concentrations of probiotics and subsequent probiotic biofilms may make the fiber surface less prone to pathogen adherence in the context of existing infections. Furthermore, in terms of treating or preventing future infections, probiotic adhesion and growth within fibers may make the environment less amenable to sustaining pathogen viability. Therefore, probiotic fibers may have the ability to both release and localize the growth of probiotics to prevent G. vaginalis and other pathogenic bacteria involved in BV and more general delivery vehicle administration.

In combination with measuring probiotic release, D- and L-lactic acid production and $\mathrm{pH}$ changes resulting from fiber administration were evaluated, due to the known antimicrobial and immunomodulatory effects of lactic acid. Lactic acid production has also been shown to restore vaginal acidity, which is another mechanism by which to inhibit pathogen viability and growth. It has been shown that both $\mathrm{D}$ - and L- isomers play important role in enhancing vaginal health, however, D- lactic acid is more protective against BV, relative to L-lactic acid, and only D- lactic acid can enhance $\alpha$-amylase activity to support Lactobacillus 
colonization $^{427}$. In our study, all fiber formulations regardless of Lactobacillus type or polymer composition demonstrated linear production rates of D- and L- lactic acid, with generally higher D-lactic acid concentrations. Based on previous studies, most Lactobacillus species produce both D- and L-lactic acid, and the D- to L-lactic acid ratio varies based on species and $\operatorname{strain}^{428}$. Generally, L. crispatus has been found to produce a higher amount of lactic acid relative to other species, in addition to a higher D- to L- lactic acid ratio ${ }^{429}$. Our results showed that there was no statistical significance between D- and L-lactic acid production as a function of fiber architecture $(P>0.05)$. Furthermore, all mesh and layered fibers released more than $3 \mathrm{mg} / \mathrm{mL}(\sim 33 \mathrm{mM})$ and $2 \mathrm{mg} / \mathrm{mL}$ ( $22 \mathrm{mM})$ of D- and L-lactic acid, or $5 \mathrm{mg} / \mathrm{mL}$ lactic acid in total, every day after 2 days. These values are within therapeuticallyrelevant ranges based on the average concentration of lactic acid reported as $1.0 \%$ $\pm 0.2 \%(\mathrm{w} / \mathrm{v})$ or $10 \mathrm{mg} / \mathrm{mL}$ in Lactobacillus-dominated microflora, specified with a Nugent score of 0 to $3^{430}$. In addition to lactic acid production, a significant change in $\mathrm{pH}$ (from 6 to 3.5 or 3 ) was observed after 1 wk for all fiber formulations ( $\mathrm{P} \leq$ 0.0001) which may be attributed to lactic acid production. These values are comparable to literature-reported values for the normal vaginal $\mathrm{pH}$ of $3.5 \pm 0.3$ (range 2.8 to 4.2$)^{430}$. Based on lactic acid production and corresponding $\mathrm{pH}$ changes, probiotic mesh and layered fibers show promise in their capabilities to restore and maintain normal vaginal health.

Finally, the preliminary efficacy of probiotic fibers was assessed in vitro using a soluble co-culture assay. For each architecture, the formulation with the highest amount of probiotic release, L. crispatus mesh and layered PLGA:PEO 
(1:1), was selected for in vitro efficacy studies. Both mesh and layered probiotic fibers completely inhibited G. vaginalis growth after $24 \mathrm{hr}$, resulting in an 8- and 6.5-log decrease in G. vaginalis viability. This significant decrease in G. vaginalis viability is much higher than the minimum bactericidal criteria specified as a 3-log decrease in the number of pathogenic bacteria ${ }^{358}$. In addition, the efficacy of probiotic fibers was similar to free probiotics, showing that probiotics maintain their activity after the electrospinning process and exert a similar effect in short-term studies. While there is no other study from other groups that evaluates the efficacy of probiotic delivery platforms against $G$. vaginalis growth in vitro, there are a few in vitro studies that assess the ability of free probiotics to inhibit $G$. vaginalis $^{358,431,432}$. One study specifically investigated the inhibitory effect of $L$. crispatus at $10^{9} \mathrm{CFU} / \mathrm{mL}$ against $\mathrm{G}$. vaginalis at $10^{8} \mathrm{CFU} / \mathrm{mL}$ in vitro and showed that after $18 \mathrm{hr}$, a 5-log decrease in G. vaginalis viability was observed which is in agreement with our observations ${ }^{358}$. While limitations exist in co-culture type assays, in particular for the long-term culture of anaerobic bacteria, sustainedrelease probiotic fibers may have the potential to inhibit G. vaginalis for a longer duration, given the amount of probiotic and lactic acid release throughout all time points of the study.

Relating this platform to its potential for future clinical delivery, the polymers utilized to fabricate these fibers, PLGA and PEO, are FDA-approved, a feature that may accelerate the translation of these platforms to clinical application. When scaled for human applications, we estimate that probiotic release, given a favorable environment for probiotic proliferation, will be on the order of $\sim 10^{10} \mathrm{CFU}$ 
for a $100 \mathrm{mg}$ dosage form (after $48 \mathrm{hr}$ ) or $10^{10} \mathrm{CFU}$ per day after $48 \mathrm{hr}$, However, if needed, fibers may be easily fabricated with lower probiotic doses. In addition, while previous in vivo studies have shown the safety and efficacy of electrospun fibers for intravaginal delivery ${ }^{34,256,433,434}$, preclinical trials need to be conducted to further evaluate the efficacy of probiotic fibers for intravaginal applications. In ongoing work, we plan to characterize the cellular and microbial responses associated with the administration of probiotic fibers in cell monolayers and EpiVaginal ${ }^{\mathrm{TM}}$ tissue. In parallel, the safety and efficacy of probiotic mesh and layered fibers will be evaluated in an established murine model of BV infection.

Overall, this study showed for the first time that electrospun fibers have the potential to enable sustained probiotic release from and corresponding proliferation in the fibers, while producing lactic acid at concentrations relevant to restoring and maintaining vaginal health. Our in vitro results also suggest that mesh and layered fibers may offer conducive architectures to foster probiotic growth and integration, similar to eukaryotic cell behavior in tissue engineering applications. In future work, we anticipate that this platform may be utilized for broader, multipurpose applications to simultaneously protect women against viral STIs, bacterial infections, and unwanted pregnancies. With this demonstrated capability of probiotic delivery, this platform may facilitate more rapid restoration of vaginal health in the context of bacterial infections, contributing to the potential to address multiple unmet needs in women's reproductive health, while providing an alternative that requires less frequent administration to help address user adherence ${ }^{435}$. 


\section{CHAPTER 6}

\section{FIRST ELECTROPHYSIOLOGICAL MEASUREMENTS CONFIRM SUCCINYL LINKER ENHANCES PERFORMANCE OF HEPTAPEPTIDE SYNTHETIC CHLORIDE CHANNEL}

\section{Introduction}

A deepening understanding of the mechanisms surrounding natural membrane transport has inevitably led to efforts at engineering such systems. Applications for such systems include highly selective filters for a specific anion or cation, a wide range of molecular sensors, novel antibiotics, drug delivery as well as DNA and RNA sequencing ${ }^{436,437}$. This study is focused on a group of chloride channels termed Synthetic Chloride Membrane TRansporters (SCMTR) ${ }^{438,439}$.

The general form of the standard SCMTR is $\left(\mathrm{R}_{1}\right)_{2} \mathrm{NCOCH}_{2} \mathrm{OCH}_{2} \mathrm{CO}-(\mathrm{Gly})_{x}-$ Pro- $(\text { Gly })_{x}-R_{2}{ }^{440}$. The dialkylamine $\left(R_{1}\right)_{2} N-$ group mimics the twin fatty acid chains of a phospholipid and serves to anchor the channel to the membrane. The linker of diglycolic acid has intermediate hydrophobicity and mimics the glycerol found in natural lipids. There is also evidence that it interacts with anions as they pass through the channel ${ }^{441}$. The amino acid sequence, $(\text { Gly })_{x}-$ Pro- $(\text { Gly })_{x}$, functions as the primary anion selectivity filter and channel with the motif design driven by 
mimicking the conserved $\mathrm{CLC}^{1}$ GxxP sequence ${ }^{442}$ and the expense of synthesizing the molecules ${ }^{443}$. The $R_{2}$ group is also a hydrophobic moiety such as a phenyl or alkane and, as with the $\mathrm{N}$-terminal alkyls, have been shown to act as a membrane anchor. The precise mechanisms that allow for such small molecules to organize and form discrete, sharply defined conductance states are unknown. There is, however, strong evidence that two or more SCMTR molecules aggregate to create multimers within the membrane to form the channels ${ }^{444,445}$.

\section{Results and Discussion}

We investigated four types of SCMTR molecules using planar lipid bilayer measurements in membranes, measuring three lipid systems and two polymers. The geometry-equilibrated structure of SCMTR molecules used in this study and their chemical formulas are shown in Figure 6.1. In this group, all of the SCMTR molecules contain a succinyl group $\left(-\mathrm{COCH}_{2} \mathrm{CH}_{2} \mathrm{CO}\right.$-) as the linker between the $\mathrm{N}$-terminal alkanes and amino acids. This structure differs from the standard form where the linker is derived from diglycolic anhydride ${ }^{439}$ that was chosen as it is structurally similar to the glycerol $\left(-\mathrm{COCH}_{2} \mathrm{OCH}_{2} \mathrm{CO}-\right)$ found in natural lipids and thus has similar polarity ${ }^{438,446}$. In addition, diglycolic acid is easily reacted with the diamines ${ }^{447}$ of the hydrophobic tail; however, when Pajewski et al. ${ }^{441}$, investigated the effect of several different linkers on the rate of chloride release from liposomes using fluorescent probes, they found that a succinate acid derivative (succinyl) had the highest chloride release rate as well as the fastest formation of ion

\footnotetext{
${ }^{1}$ There appears to be some confusion in the literature over abbreviation for the chloride channel. Early literature abbreviated with a lower case " $\mathrm{L}$ " as in chlorine from the periodic table. The recent trend however is to use an upper case " $\mathrm{L}$ " so it is not confused with an upper case "i".
} 
conductance pathways ${ }^{441}$. To our knowledge, this is the first report of planar bilayer experiments with this linker.

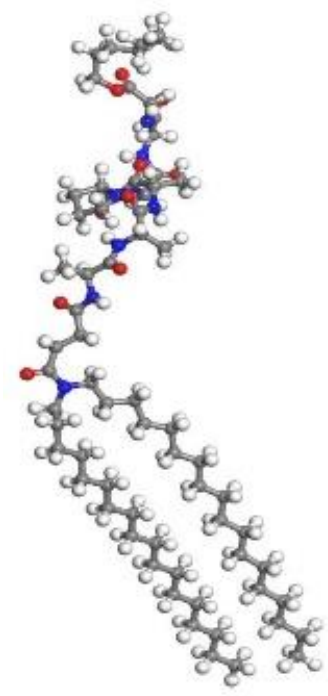

SCMTR\#1 SCMTR\#2
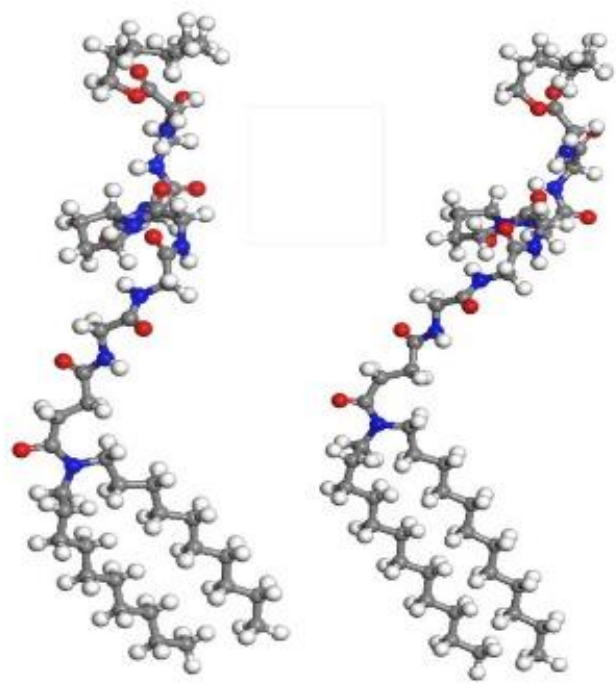

SCMTR\#3 SCMTR\#4

Figure 6.1. Molecular structure of different compounds used in the experiment. The chemical formula of each is listed below.

$\left(\mathrm{C}_{18} \mathrm{H}_{37}\right)_{2}-\mathrm{N}-\mathrm{OC}-\mathrm{CH}_{2}-\mathrm{CH}_{2}-\mathrm{CO}-\mathrm{AAAPGGG}-\mathrm{O}\left(\mathrm{CH}_{2}\right)_{6} \mathrm{CH}_{3} \mathrm{Mw}=1201.75 \mathrm{~g} / \mathrm{mol}$ $\left(\mathrm{C}_{8} \mathrm{H}_{17}\right)_{2}-\mathrm{N}-\mathrm{OC}-\mathrm{CH}_{2}-\mathrm{CH}_{2}-\mathrm{CO}-\mathrm{GGGPGGG}-\mathrm{O}\left(\mathrm{CH}_{2}\right)_{6} \mathrm{CH}_{3} \quad \mathrm{Mw}=879.15 \mathrm{~g} / \mathrm{mol}$ $\left(\mathrm{C}_{10} \mathrm{H}_{21}\right)_{2}-\mathrm{N}-\mathrm{OC}-\mathrm{CH}_{2}-\mathrm{CH}_{2}-\mathrm{CO}-\mathrm{GGGPGGG}-\mathrm{O}\left(\mathrm{CH}_{2}\right)_{6} \mathrm{CH}_{3} \quad \mathrm{Mw}=935.26 \mathrm{~g} / \mathrm{mol}$ $\left(\mathrm{C}_{12} \mathrm{H}_{25}\right)_{2}-\mathrm{N}-\mathrm{OC}-\mathrm{CH}_{2}-\mathrm{CH}_{2}-\mathrm{CO}-\mathrm{GGGPGGG}-\mathrm{O}\left(\mathrm{CH}_{2}\right)_{6} \mathrm{CH}_{3} \quad \mathrm{Mw}=991.35 \mathrm{~g} / \mathrm{mol}$

Each design in the set of SCMTR molecules explored here contains a variant of the $\mathrm{R}_{1} \mathrm{~N}$-terminal domain; $\mathrm{C}_{18} \mathrm{H}_{37}, \mathrm{C}_{8} \mathrm{H}_{17}, \mathrm{C}_{10} \mathrm{H}_{21}$, and $\mathrm{C}_{12} \mathrm{H}_{25}$. The effects of such variations have been explored in the past, though only for the case of diglycolic acid linkers. In one of the most complete studies, the relative chloride release rates from liposomes with $\mathrm{C}_{3}$ to $\mathrm{C}_{18} \mathrm{~N}$-terminated SCMTR molecules were 
measured using chloride selective electrodes and fluorescence techniques ${ }^{448}$. The result was that $\mathrm{C}_{8}$ produced the most rapid ion transport. The order of release rate was $\mathrm{C}_{8}>\mathrm{C}_{10}>\mathrm{C}_{6}>\mathrm{C}_{12}>\mathrm{C}_{3}=\mathrm{C}_{14}=\mathrm{C}_{18}>\mathrm{C}_{16}$ and the $C$-terminal in this case was a benzyl. A second study ${ }^{449}$ explored both variation of the $\mathrm{N}$ - and $\mathrm{C}$ - terminal portions of the molecule using a chloride selective electrode and fluorescence data. Interestingly, it showed that for the case where the $C$-terminal was a heptyl instead of a benzyl, the shortest $N$-terminal was most active with $\mathrm{C}_{6}>\mathrm{C}_{10}>\mathrm{C}_{18}$. For the benzyl-terminated molecules they found that release rates were $C_{10}>C_{6}>C_{18}$, which was consistent with the first study.

The role of the $C$-terminal side of the molecules $\left(R_{2}\right)$ in chloride conduction was investigated in previous work 447,450 by examining structures that included alkanes with lengths from $\mathrm{C}_{2}$ to $\mathrm{C}_{18}$ with an ether linkage, alkanes with an amine linkage, diacylamides (e.g. $\left.-\mathrm{N}\left[\left(\mathrm{CH}_{2}\right)_{9} \mathrm{CH}_{3}\right]_{2}\right)$ and the canonical benzyl. It was generally found that anion release rates from liposomes were highest for linear alkanes of length $\mathrm{C}_{7}$ and the didecylamide $\left(-\mathrm{N}\left[\left(\mathrm{CH}_{2}\right)_{9} \mathrm{CH}_{3}\right]_{2}\right)$. Thus, it is evident that both anchor chains are instrumental in channel formation. As with the $\mathrm{N}$-terminal, it is surprising that the conductivity is maximized for chain lengths that are so much shorter than the fatty acid found in lipids, for example, DOPC contains 18 carbons. Another striking study indicated that the binding constant of the (Gly)3-Pro-(Gly)3 is not affected by the length of alkyl groups ${ }^{450}$ and concentration studies using NMR implied no aggregation (no dimers or greater), however, the experiments were performed in $\mathrm{CDCl}_{3}$ (deuterated chloroform) and, therefore, in relatively different electrostatic environments. 
All SCMTR molecules were synthesized by Dr. George Gokel's group at the University of Missouri (St. Louis) via previously reported procedures ${ }^{438,439,447,451}$. They were then dissolved in DMSO $(\sim 0.45 \mathrm{mg} / \mathrm{ml})$ and tested in asolectin (50 $\mathrm{mg} / \mathrm{ml})$, DOPC: egg PE (20 mg/ml in decane) and DPhPC (20 mg/ml in decane). SCMTR 1 containing the amino acid sequence AAAPGGG showed the least activity of the molecules tested. Given that alanine has only a methyl side group, it is regarded as a hydrophobic amino acid. Thus, it is not surprising that a lack of activity from the AAAPGGG sequence was observed since the nonpolar alanine interacting in the channel likely made ion passage energetically unfavorable. SCMTR 2 exhibited ion channel behavior in all cases except DPhPC and was most active in egg PC. In BLM experiments with asolectin, conductance states of $12.2 \pm 2.5 \mathrm{pS}$ and $19.3 \pm 2.6 \mathrm{pS}$ at $90 \mathrm{mV}$ were observed and in the egg PC system, two conductance states of $4.7 \pm 2.1 \mathrm{pS}$ and $19.4 \pm 2.1 \mathrm{pS}$ were detected at $150 \mathrm{mV}$. SCMTR 3 was more active than SCMTR 2. The two conductance values of $18.2 \pm 1.4 \mathrm{pS}$ and $25.6 \pm 2.8 \mathrm{pS}$ were obtained at $120 \mathrm{mV}$ in asolectin.

SCMTR 4 was generally the most active ion channel tested, thus we observed the largest number of channel open events for a given concentration. As shown in Figure 6.2, at $-65 \mathrm{mV}$ two conductance states of $15.5 \pm 4.1 \mathrm{pS}$ and $27.8 \pm 3.1 \mathrm{pS}$ were achieved in asolectin. While similar states of $18.8 \pm 1.6$ and $20.3 \pm 1.9$ pS were observed in DOPC:egg PE system at $130 \mathrm{mV}$. This molecule was also inactive in DPhPC membranes. 

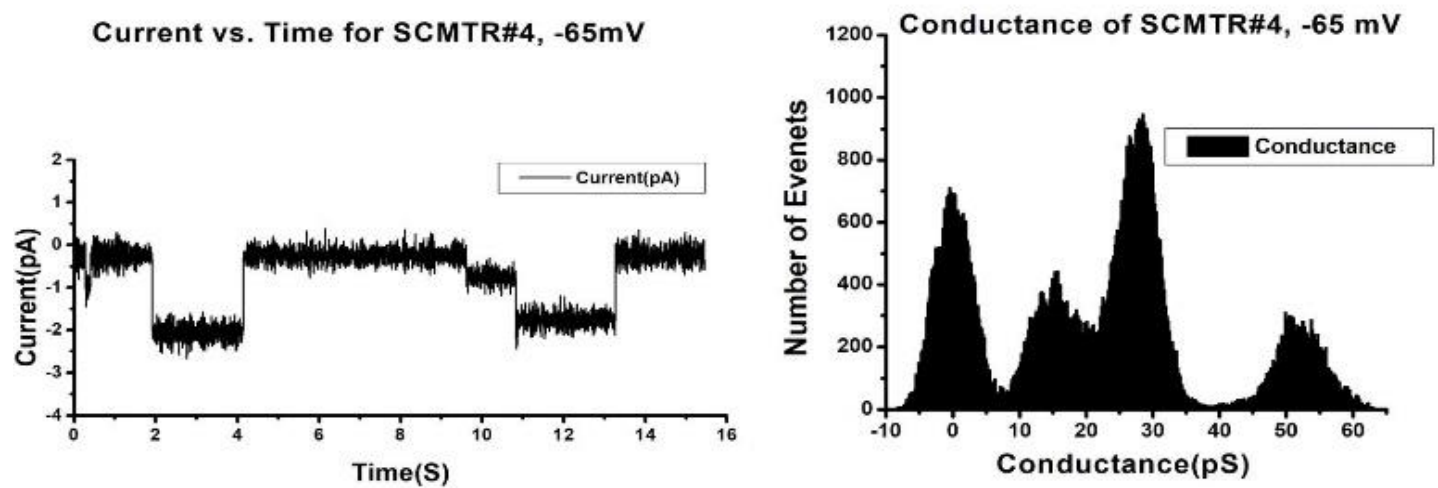

Figure 6.2. Current vs. time with a $100 \mathrm{~Hz}$ low pass Bessel filter (left) and histogram of conductance (right) for 4 at $-65 \mathrm{mV}$. Two conductance states of 15.5 $\pm 4.1 \mathrm{pS}, 27.8 \pm 3.1 \mathrm{pS}$ and the linear combination of the second state $(52.0 \pm 3.8$ pS) are indicated.

The selectivity of SCMTR 4 was investigated by carefully replacing the $0.5 \mathrm{M} \mathrm{KCl}$ electrolyte with $0.5 \mathrm{M} \mathrm{K}_{2} \mathrm{SO}_{4}$ at the same $\mathrm{pH}$ on one side of the membrane (trans compartment). Then the polarity of the applied voltage was switched from $-50 \mathrm{mV}$ to $+50 \mathrm{mV}$ to select anion flow from cis to trans compartments. Figure 6.3 illustrates the result of SCMTR selectivity to chloride ions over sulfate ions. Two conductance states were observed for both anions and measurements of the highest conductance state for each gives $6.9 \pm 2.0 \mathrm{pS}$ for sulfate and 20.5 $\pm 3.2 \mathrm{pS}$ for chloride. Thus, the $\mathrm{Cl}^{-}$gave a 3-fold higher conductance than $\mathrm{SO}_{4}{ }^{2-}$. 


\section{Selectivity measurements for SCMTR\#4}

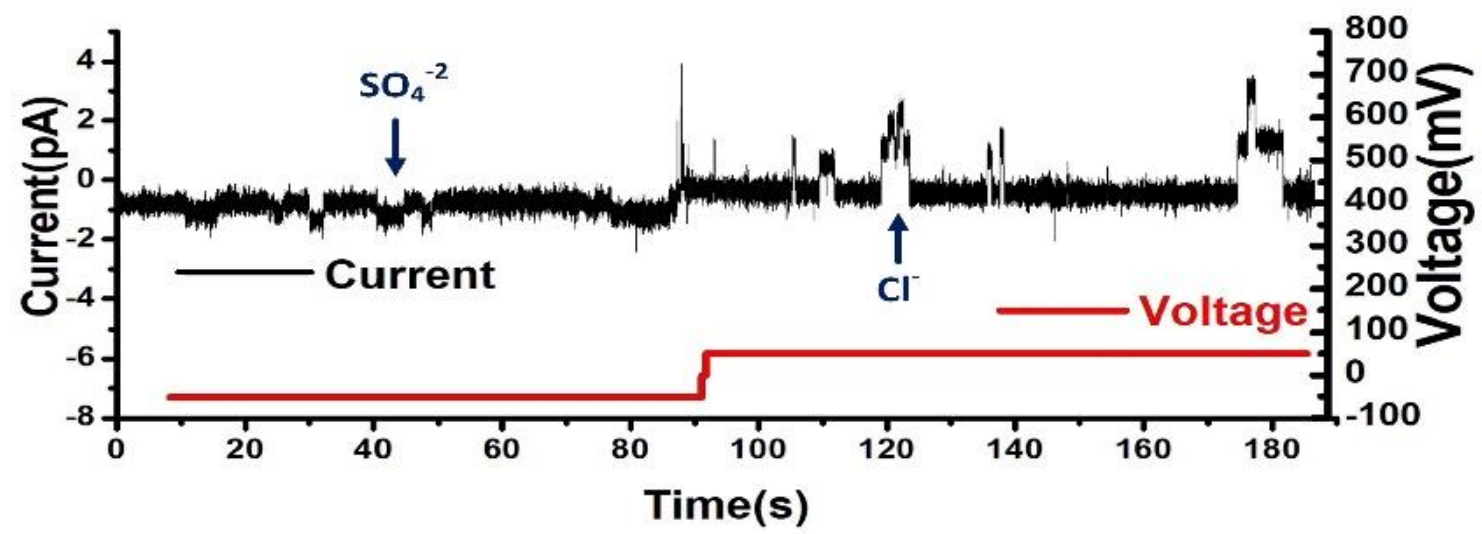

Figure 6.3. Current vs. time plot after replacing $\mathrm{KCL}$ with $\mathrm{K}_{2} \mathrm{SO}_{4}$ in trans compartment. The highest conductance state at positive and negative polarity is $6.9 \pm 2.0 \mathrm{pS}$ and $20.5 \pm 3.2 \mathrm{pS}$, respectively.

Molecules $\mathbf{3}$ and $\mathbf{4}$, i.e the most active, were tested in biomimetic membrane block co-polymers of poly(2-methyl-2-oxazoline) (PMOXA) and poly(dimethyl siloxane) (PDMS). Specifically, the diblock (PMOXA 6 -PDMS ${ }_{17}$ ) and the triblock $\left(\mathrm{PMOXA}_{6}-\mathrm{PDMS}_{35}-\mathrm{PMOXA}_{6}\right)$ were examined as these materials have demonstrated reconstitution of alpha-hemolysin, alamethicin and gramicidin ion channels ${ }^{452,453}$. No activity was observed in these cases.

Three out of the four SCMTR molecules tested exhibited channel activity and in those cases at least two unique, well defined conductance states were observed. Typically, the first conductance state was between 4 and $18 \mathrm{pS}$ while the second lay between 18 and $28 \mathrm{pS}$. The activity increased as the $\mathrm{N}$-terminal

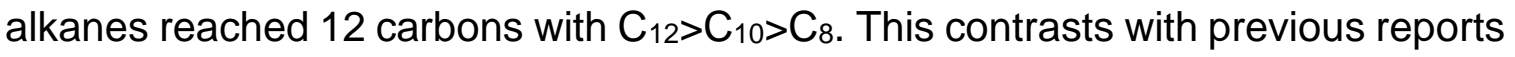
of molecules having diglycolic acid (- $\left.\mathrm{COCH}_{2} \mathrm{OCH}_{2} \mathrm{CO}-\right)$ as a linker that showed 
activities following the sequence $\mathrm{C}_{6}>\mathrm{C}_{10}>\mathrm{C}_{18}$ instead of the succinyl ($\mathrm{COCH}_{2} \mathrm{CH}_{2} \mathrm{CO}$-). The values showed weak dependence on the membrane composition and there was no clear indication of voltage dependent gating. It was further noteworthy that the activity of the samples seemed to degrade rapidly after being solvated in DMSO with samples losing all trace of channel behavior after about 2 weeks when stored at $4^{\circ} \mathrm{C}$.

While the exact mechanism for the formation of conductance channels by small molecules such as the SCMTR analogs remains an open question, recent work modifying the $\beta$-barrel ${ }^{436}$ of alpha-hemolysin to shorten the transmembrane section below the thickness of the lipid membrane have shown that it is still possible to obtain channel behavior. It is suggested ${ }^{436}$ that pores in such cases are formed by buckling of the lipids around the protein to form a hydrophilic ring. Further, molecular dynamics simulations of canonical SCMTR molecules by Burkhardt et al. ${ }^{454}$ support the hypothesis of membrane reorganization for the formation of water channels through the membrane and predicts conductance states with values between 55 and $126 \mathrm{pS}$.

Appreciation is expressed for the financial support of this work from the $\mathrm{J}$. B. Speed College of Engineering, University of Louisville. We also acknowledge Dr. George Gokel at the University of Missouri (St. Louis) who provided the SCMTR samples. 


\section{CHAPTER 7}

\section{OVERALL DISCUSSION AND CONCLUSION}

One-third of the global health issues and disease burden for women of age 15-44 years is attributed to poor sexual and reproductive health outcomes ${ }^{455}$. These health burdens are mostly attributed to female reproductive viral and bacterial infections, such as HIV-1, HSV-2, and BV. These infections can enhance virus shedding in patients, stimulate immune cell infiltration to the localized region, and promote microscopic tears that enable epithelial and immune cells to be more susceptible to infection, thereby increasing the risk of HIV and HSV-2 co-infections. Given the diversity and magnitude of these unmet reproductive health needs, there is an urgent need to develop novel topical delivery platforms with multipurpose prevention that simultaneously target a diversity of sexually transmitted pathogens ${ }^{4}$.

The overall goal of this work was to develop novel strategies to enhance the delivery and efficacy of active agents, including live probiotic cells, from intravaginal delivery platforms. In addition, these dosage forms including NPs, NPfiber composites, and dual-electrospun mesh and layered fibers were designed and developed to help overcome challenges associated with current topical delivery platforms which include: transient release, inefficient loading, frequent 
application, and low user adherence. Finally, these studies have shown that these delivery platforms can offer a safe and efficacious alternative to women with the potential to encapsulate a variety of agents ranging from small molecules to large proteins and even live probiotic cells.

Combination therapy is one of the most promising strategies to decrease the global prevalence of STIs. A variety of ARVs have been developed to prevent HIV infection either before or after HIV exposure. While approximately 25 anti-HIV drugs currently exist, only a few of them, such as valaciclovir, foscarnet, and raltegravir, are dually efficacious against HSV-2 infection. One of the challenges with ARVs, currently used in treatment regimens, is the increased risk of drugresistance and the corresponding long-term side effects of administration. One option to help overcome these challenges is in the discovery and development of efficacious biological molecules, that have a demonstrated safety profile and are known to exert functional effect via different mechanisms of action. One of these candidates, GRFT, is as an antiviral lectin that has been shown to prevent both HIV-1 and HSV-2 infections and is currently in Phase I clinical trials against HIV-1 infection ${ }^{456}$. Additionally, GRFT exhibits an excellent safety profile, with negligible induction of pro-inflammatory cytokines ${ }^{231,457}$ and has demonstrated synergy with other antiretrovirals ${ }^{222}$, suggesting the benefits of future co-administration strategies.

In this work, we sought to develop new multipurpose formulations, comprised of Q-GRFT (an oxidation-resistant variant of GRFT) and an ARV (e.g. TFV, RAL, or DAP) to enhance the efficacy of active agents in preventing against 
HIV-1 infection. These ARVs were selected due to their distinct chemical properties and different mechanisms of action and Q-GRFT was used as a promising biological candidate with potent antiviral properties. First, we showed that all active agents were successfully encapsulated in PLGA NPs with loading efficiency values in the range of 15 to $100 \%$. In addition, all NP formulations provided $\sim 20 \%$ release over two weeks in SVF. Next, each ARV, in free and encapsulated forms, was co-administered with Q-GRFT or Q-GRFT NPs to evaluate the impact of co-administration on prophylactic efficacy against HIV-1 in vitro. The in vitro efficacy of free versus encapsulated agents was subsequently evaluated against HIV-1 pseudovirus infection, showing that Q-GRFT NPs were 4.1-fold more efficacious, relative to free Q-GRFT, while ARV-encapsulated NPs had similar $\mathrm{IC}_{50}$ values to the administration of each free ARV alone. In parallel, the potential of Q-GRFT and ARV co-administration in both free and encapsulated forms were evaluated. When free Q-GRFT was co-administered with each free ARV, synergistic interactions were observed for all co-administration groups with a 3.7 to 6.3-fold decrease in the IC 50 of free Q-GRFT and a 2.8 to 5.6 -fold reduction in the IC 50 values of free ARVs. When Q-GRFT and ARVs were each encapsulated in NPs, synergistic interactions were observed for all formulation combinations. Specifically, the synergy between Q-GRFT- and DAP-encapsulated NPs significantly improved, relative to free Q-GRFT and free DAP co-administration, with 6 and 8.6-fold increases in efficacy, respectively.

Based on these results, we selected the most synergistic co-administered formulations, Q-GRFT NPs and DAP NPs, and aimed to investigate the impact of 
incorporating Q-GRFT and DAP in NP-fiber composites. DAP, a small hydrophobic molecule, showed a burst release followed by minimal release that was still within the in vitro $\mathrm{IC}_{50}$ range previously observed with DAP administration to TZM-bl cells. In comparison, the release profile of Q-GRFT, a large generally hydrophilic protein, followed an "S" shape, indicating two distinct release phases on day 1 and days 42 through 60 , each of which was followed by more gradual release. While the release profiles of these specific agents, provided by this platform, were less than ideal for prolonged delivery applications, it was worthwhile to investigate the incorporation and release of active agents with different hydrophilicities and sizes in a NP-fiber composite. In future work, the release profiles may be optimized by changing the NP content, outer layer polymer composition and thickness, and fiber diameter.

In order to obtain a better understanding of the potential for polymeric dosage forms to incorporate ARVs, we used computational modeling to study intermolecular interactions between PLGA and TFV, RAL, and DAP. Molecular dynamic and molecular mechanic simulations indicated that hydrogen bonding between TFV and PLGA in the presence of water was weaker than between TFV and water in the same system, causing the drug to leach into the aqueous phase in the process of NP fabrication and causing low encapsulation efficacy. However, hydrogen bonding between the oxygen atom in the carbonyl group of PLGA and the hydrogen atom connected to the oxygen atom in RAL was stronger than RAL and the oxygen atom in water, resulting in high encapsulation efficacy for RAL 
NPs. These results are in agreement with experimental loading values for TFV and RAL which were 15 and $100 \%$.

In parallel with concerns of viral reproductive infections, bacterial infections, such as $\mathrm{BV}$, present the most frequent vaginal infection affecting around $33 \%$ of women, domestically and globally $6,334,458$ and have been shown to increase the risk of HIV-1 acquisition by up to $60 \%$. Clinical studies have shown that BV may be responsible for a significant increase in reported HIV-1 infections in Africa ${ }^{7}$ and that women infected with BV have a higher risk of infecting their male partner. Balancing and restoring the vaginal flora using probiotic delivery may both treat BV and lessen female-to-male HIV-1 transmission. Therefore, two studies in this dissertation focused on developing novel probiotic dosage forms to potentially prevent and treat BV infection and recurrence.

Traditional therapies such as oral and topical antibiotics have been used for BV treatment, showing an initially high treatment efficacy of $80 \%$. However, there are challenges associated with antibiotic therapies including the risk of BV recurrence and the development of antibiotic-resistant bacteria. One promising approach is to use probiotics, such as Lactobacillus, to reduce BV symptoms and improve the endogenous vaginal microflora ${ }^{326-328}$, thereby "priming" the FRT for beneficial bacteria colonization and proliferation. Probiotic delivery for BV treatment has been preliminary explored with the focus on relatively transient therapy, that requires daily administration. Current dosage forms, including gels, films, and tablets, used to deliver probiotics to the FRT are associated with low intravaginal residence time, which may result in transient protection, messiness 
via leakage, and unfavorable discharge that can adversely impact user adherence and efficacy. Next-generation delivery platforms seek to address these challenges by providing long-term prophylactic and therapeutic alternatives.

In these studies, we first demonstrated the safety and functional impact of blank and antibiotic rapid-dissolve PEO fibers for treatment in a murine model of BV infection. In vivo experiments showed that PEO fibers were relatively inert in the mouse vagina and did not adversely affect the tissue. Moreover, fibers that incorporated $100 \mu \mathrm{g} / \mathrm{mg}$ fiber metronidazole were capable of both preventing and treating $G$. vaginalis infection in a mouse model. From a materials perspective, the effect of different parameters including electrospinning solution, polymer type, and the incorporation of fresh or lyophilized probiotics on post-spin viability was assessed. Results showed that electrospinning solution and polymer type have minimal impact on probiotic viability, whereas the choice of fresh or lyophilized bacteria exerted a significant impact on probiotic viability. Overall, using fresh bacteria resulted in 4-logs higher viability, relative to the incorporation of lyophilized bacteria. In addition to evaluating post-spun probiotic viability, the long-term viability of probiotic fibers in different storage conditions was examined for up to $90 \mathrm{~d}$. When fibers were stored at room temperature, L. acidophilus viability decreased dramatically over the first few days, resulting in negligible viability after one month. Among all formulations, the highest $L$. acidophilus viability after 3 months was observed in PEO fibers that were electrospun in MRS with glycerol and stored at $-20^{\circ} \mathrm{C}$. Lastly, for the first time, we showed that $L$. acidophilus PEO and PVA fibers administered at $10^{7}$ and $10^{8} \mathrm{CFU} / \mathrm{mL}$ ( 2 and $20 \mathrm{mg}$ of fiber per 
$\mathrm{mL}$ media based on actual loading) can fully inhibit $G$. vaginalis growth $\left(10^{8}\right.$ $\mathrm{CFU} / \mathrm{mL}$ ) after $24 \mathrm{hr}$ co-culture. Moreover, fibers at both concentrations completely inhibited G. vaginalis adherence to HeLa cells after $16 \mathrm{hr}$.

Based on the promising results of this study, we sought to build upon these rapid-dissolve formulations to fabricate more complex fiber architectures composed of both hydrophilic and hydrophobic polymers to modulate probiotic release. Both mesh and layered architectures incorporating $L$. crispatus or $L$. acidophilus demonstrated high, therapeutically-relevant levels of probiotic release and lactic acid production for up to $14 \mathrm{~d}$, resulting in a significant reduction in $\mathrm{pH}$. In addition, soluble co-culture experiments showed that both mesh and layered fibers incorporating probiotics completely inhibit G. vaginalis growth after $48 \mathrm{hr}$. These results suggest that mesh fibers may offer a viable long-term probiotic alternative to daily antibiotic administration to maintain vaginal health, treat $\mathrm{BV}$, and prevent BV recurrence.

\section{Conclusion and Future Work}

All of the presented studies showed promise to enhance the intravaginal delivery of agents to the FRT; however, moving forward, there are a variety of directions to pursue in future work. Regarding the NP delivery platforms developed for HIV-1 prevention, all of the selected ARVs were co-administered with Q-GRFT at equipotent ratios and demonstrated synergy. However, other ratios may be explored in future work to further increase synergy, in particular for NPencapsulated agents which may require loading and releaseoptimization as well. 
In addition to the ratio of Q-GRFT:ARV, different administration times may be explored to optimize the efficacy of these active agents for utility in both prevention and treatment applications. In this study, the active agents were administered or co-administered $1 \mathrm{hr}$ prior to virus administration which indicates their potential utility for prevention. However, due to the different mechanisms of action of these ARVs, administration of NPs simultaneous to or after virus infection may also demonstrate therapeutic effect against HIV-1 infection. Therefore, in future experiments, different temporal regimens may be evaluated to similarly assess efficacy and synergistic interactions between Q-GRFT and different ARVs. In addition to these in vitro experiments, in vivo experiments, which explore the impact of co-administration, may be conducted to determine release, distribution, retention, and corresponding efficacy of these active agents and delivery platforms as translated to an initial murine model of HIV-1 infection.

In addition to NP co-administration, we demonstrated the potential of NPfiber composites to alter Q-GRFT and DAP release profiles; however, the in vitro efficacy was not explored. Therefore, the efficacy of NP-fiber composites that incorporate both Q-GRFT and DAP against HIV-1 infection may be explored in future work. Furthermore, the release profile for these and other active agents may be optimized by changing the NP content and altering the outer layer fiber thickness and polymer type.

As we showed in our studies, PLGA NPs encapsulate ARVs and proteins with moderate loading. However, there are some challenges regarding the encapsulation of small hydrophilic molecules such as TFV. Molecular dynamic 
simulations demonstrated that due to the stronger hydrogen bonding between TFV and water molecules, PLGA NPs cannot encapsulate TFV. Therefore, adding more reactive moieties to PLGA chains may improve the intermolecular interactions between the polymer and the drug, leading to higher loading. In the future, the effect of adding different functional groups, such as polyethylene glycol, to the polymer chain may be evaluated to tailor the interactions between TFV and PLGA, and to improve the loading of NPs.

In addition to HIV-1 prevention, we aimed to develop a new delivery platform to treat BV infection. The first-generation rapid release PEO and PVA fibers incorporated probiotics with relatively high viability and stability under refrigerated and freezer conditions. However, probiotic viability and stability under different storage conditions may be further improved by incorporating other and more concentrated nutrient sources such as inulin, skim milk, and lactulose. Due to the preliminary in vitro efficacy observed with rapid-dissolve hydrophilic fibers, we evaluated more complex fiber architectures to provide a prolonged probiotic release to treat $\mathrm{BV}$ recurrence.

While rapid-release fibers demonstrated initial promise in providing ondemand protection against $G$. vaginalis infection, sustained-release probiotic fibers consisting of hydrophilic and hydrophobic polymers are anticipated to provide longterm treatment for BV and may provide a new option to help restore the balance of beneficial bacteria to the FRT. Furthermore, one may envision that these fibers may be used prophylactically to help maintain female reproductive health in women who are considered at-risk for recurrent BV infections. In this work, both 
mesh and layered fibers, comprised of PLGA and PEO were successfully fabricated to incorporate high concentrations of probiotics and to promote the sustained-release and localized proliferation of probiotics through two weeks. Electrospun fibers such as these, may offer women an alternative delivery platform that can provide localized delivery for a prolonged period of time, prompting less frequent administration by patients, which may enhance user adherence. Moreover, this delivery platform provided the opportunity to consider other technologies that may be suitable to even longer time frames of probiotic release, potentially offering women more alternatives.

One potential direction of future work is to investigate the feasibility of fibers as multipurpose delivery platforms to deliver both probiotics and antiviral agents simultaneously to protect women against both BV recurrence and HIV-1 infection. Mention the possibility of antibiotic then antiviral phased administration - in this or another platform design. From a release perspective, even though the delivery platform we developed demonstrated promise in sustaining probiotic release, other polymer compositions such as PCL and fiber architectures such as coaxial fibers may be explored to further enhance this delivery platform. Furthermore, due to the promising in vitro results regarding the efficacy of probiotic-containing fibers, we plan to examine their safety and efficacy in vaginal epithelial cells, EpiVaginal ${ }^{\mathrm{TM}}$ tissue, and in a more complex in vivo model. By doing so, we anticipate attaining a more in-depth understanding of host, microbe, and vehicular interactions via the characterization of cellular and microbial responses associated with probiotic administration. 


\section{REFERENCES}

1 Garcia-Vidal, E. et al. Dual effect of the broad spectrum kinase inhibitor midostaurin in acute and latent HIV-1 infection. Antiviral research 168, 18-27 (2019).

2 Gama, L. \& Koup, R. A. New-generation high-potency and designer antibodies: role in HIV-1 treatment. Annual review of medicine 69, 409-419 (2018).

3 Baeten, J. M. et al. Use of a vaginal ring containing dapivirine for HIV-1 prevention in women. New England Journal of Medicine 375, 2121-2132 (2016).

4 Boonstra, H., Barot, S. \& Lusti-Narasimhan, M. Making the case for multipurpose prevention technologies: the socio-epidemiological rationale. BJOG : an international journal of obstetrics and gynaecology 121 Suppl 5, 23-26, doi:10.1111/1471-0528.12851 (2014).

5 Jones, A. Bacterial Vaginosis: A Review of Treatment, Recurrence, and Disparities. The Journal for Nurse Practitioners 15, 420-423 (2019).

6 Peebles, K., Velloza, J., Balkus, J. E., McClelland, R. S. \& Barnabas, R. V. High global burden and costs of bacterial vaginosis: a systematic review and metaanalysis. Sexually transmitted diseases 46, 304-311 (2019).

7 Cohen, C. R. et al. Bacterial vaginosis associated with increased risk of female-tomale HIV-1 transmission: a prospective cohort analysis among African couples. PLoS Med 9, e1001251 (2012).

8 Sewankambo, N. et al. HIV-1 infection associated with abnormal vaginal flora morphology and bacterial vaginosis. Lancet 350, 546-550 (1997).

9 Jamieson, D. J. et al. Longitudinal analysis of bacterial vaginosis: findings from the HIV epidemiology research study. Obstet Gynecol 98, 656-663 (2001).

10 McKinnon, L. R. et al. The evolving facets of bacterial vaginosis: implications for HIV transmission. AIDS research and human retroviruses 35, 219-228 (2019).

11 Verstraelen, H., Verhelst, R., Vaneechoutte, M. \& Temmerman, M. The epidemiology of bacterial vaginosis in relation to sexual behaviour. BMC infectious diseases 10, 1-11 (2010).

12 Wira, C. R., Patel, M. V., Ghosh, M., Mukura, L. \& Fahey, J. V. Innate immunity in the human female reproductive tract: endocrine regulation of endogenous antimicrobial protection against HIV and other sexually transmitted infections. Am $J$ Reprod Immunol 65, 196-211, doi:10.1111/j.1600-0897.2011.00970.x (2011).

13 Unnithan, A. R. et al. Wound-dressing materials with antibacterial activity from electrospun polyurethane-dextran nanofiber mats containing ciprofloxacin $\mathrm{HCl}$. Carbohydr Polym 90, 1786-1793 (2012). 
14 Tourgeman, D. E., Gentzchein, E., Stanczyk, F. Z. \& Paulson, R. J. Serum and tissue hormone levels of vaginally and orally administered estradiol. American journal of obstetrics and gynecology 180, 1480-1483 (1999).

15 Steinbach, J. M. Protein and oligonucleotide delivery systems for vaginal microbicides against viral STIs. Cellular and Molecular Life Sciences 72, 469-503, doi:10.1007/s00018-014-1756-3 (2015).

16 Bernkop-Schnürch, A. \& Hornof, M. Intravaginal drug delivery systems. American journal of drug delivery 1, 241-254 (2003).

17 Chou, S. F., Carson, D. \& Woodrow, K. A. Current strategies for sustaining drug release from electrospun nanofibers. J Control Release 220, 584-591, doi:10.1016/j.jconrel.2015.09.008 (2015).

18 Hickey, D. K., Patel, M. V., Fahey, J. V. \& Wira, C. R. Innate and adaptive immunity at mucosal surfaces of the female reproductive tract: stratification and integration of immune protection against the transmission of sexually transmitted infections. $J$ Reprod Immunol 88, 185-194 (2011).

19 Wiggins, R., Hicks, S., Soothill, P., Millar, M. \& Corfield, A. Mucinases and sialidases: their role in the pathogenesis of sexually transmitted infections in the female genital tract. Sex Transm Infect. 77, 402-408 (2001).

20 das Neves, J. \& Bahia, M. F. Gels as vaginal drug delivery systems. Int J Pharm 318, 1-14, doi:https://doi.org/10.1016/i.ijpharm.2006.03.012 (2006).

21 Andrews, G. P. et al. Characterization of the rheological, mucoadhesive, and drug release properties of highly structured gel platforms for intravaginal drug delivery. Biomacromolecules 10, 2427-2435 (2009).

22 Devlin, B., Nuttall, J., Wilder, S., Woodsong, C. \& Rosenberg, Z. Development of dapivirine vaginal ring for HIV prevention. Antiviral Res 100, S3-S8 (2013).

Derby, N., Zydowsky, T. \& Robbiani, M. In search of the optimal delivery method for anti-HIV microbicides: are intravaginal rings the way forward? Expert Rev. Antiinfect. Ther. 11, 5-8 (2013).

$24 \mathrm{Ho}$, E. A. Intravaginal rings as a novel platform for mucosal vaccination. $\mathrm{J} \mathrm{Mol}$ Pharm Org Process Res (2013).

25 Mallipeddi, R. \& Rohan, L. C. Nanoparticle-based vaginal drug delivery systems for HIV prevention. Expert opinion on drug delivery 7, 37-48 (2010).

Kiser, P. F., Johnson, T. J. \& Clark, J. T. State of the art in intravaginal ring technology for topical prophylaxis of HIV infection. Aids Rev 14, $62-77$ (2012).

27 Dieben, T. O., Roumen, F. J. \& Apter, D. Efficacy, cycle control, and user acceptability of a novel combined contraceptive vaginal ring. Obstetrics \& Gynecology 100, 585-593 (2002).

28 Malcolm, R. K., Edwards, K.-L., Kiser, P., Romano, J. \& Smith, T. J. Advances in microbicide vaginal rings. Antiviral res 88, S30-S39 (2010).

29 Roumen, F., Apter, D., Mulders, T. \& Dieben, T. Efficacy, tolerability and acceptability of a novel contraceptive vaginal ring releasing etonogestrel and ethinyl oestradiol. Human Reproduction 16, 469-475 (2001).

30 Nel, A. et al. Safety and efficacy of a dapivirine vaginal ring for HIV prevention in women. New England Journal of Medicine 375, 2133-2143 (2016).

31 Kim, S., Traore, Y. L., Chen, Y., Ho, E. A. \& Liu, S. Switchable On-Demand Release of a Nanocarrier from a Segmented Reservoir Type Intravaginal Ring Filled with a pH-Responsive Supramolecular Polyurethane Hydrogel. ACS Appl Bio Mater. 1, 652-662 (2018).

32 Vanić, Ž. \& Škalko-Basnet, N. Nanopharmaceuticals for improved topical vaginal therapy: can they deliver? Eur. J. Pharm. Sci. 50, 29-41 (2013). 
33 Zhang, T., Sturgis, T. F. \& Youan, B.-B. C. pH-responsive nanoparticles releasing tenofovir intended for the prevention of HIV transmission. Eur J Pharm Biopharm. 79, 526-536 (2011).

34 Krogstad, E. A. et al. Nanoparticle-releasing nanofiber composites for enhanced in vivo vaginal retention. Biomaterials 144, 1-16 (2017).

35 Martínez-Pérez, B. et al. Controlled-release biodegradable nanoparticles: From preparation to vaginal applications. Eur. J. Pharm. Sci. 115, 185-195 (2018).

36 Marciello, M., Rossi, S., Caramella, C. \& Remuñán-López, C. Freeze-dried cylinders carrying chitosan nanoparticles for vaginal peptide delivery. Carbohydr Polym 170, 43-51 (2017).

37 Leyva-Gómez, G. et al. Approaches in Polymeric Nanoparticles for Vaginal Drug Delivery: A Review of the State of the Art. Int J Mol Sci 19, 1549 (2018).

38 Sims, L. B., Frieboes, H. B. \& Steinbach-Rankins, J. M. Nanoparticle-mediated drug delivery to treat infections in the female reproductive tract: evaluation of experimental systems and the potential for mathematical modeling. Int $J$ Nanomedicine 13, 2709 (2018).

39 El-Hammadi, M. M. \& Arias, J. L. Nanotechnology for Vaginal Drug Delivery and Targeting. Nanotechnology and Drug Delivery, Volume Two: Nano-Engineering Strategies and Nanomedicines against Severe Diseases, 191 (2016).

40 Ensign, L. M. et al. Mucus-penetrating nanoparticles for vaginal drug delivery protect against herpes simplex virus. Sci Transl Med 4, 138ra179, doi:10.1126/scitransImed.3003453 (2012).

41 Maisel, K. et al. Nanoparticles coated with high molecular weight PEG penetrate mucus and provide uniform vaginal and colorectal distribution in vivo. Nanomedicine 11, 1337-1343 (2016).

42 Henry, C. E. et al. Anti-PEG antibodies alter the mobility and biodistribution of densely PEGylated nanoparticles in mucus. Acta biomater. 43, 61-70 (2016).

43 Mohideen, M. et al. Degradable bioadhesive nanoparticles for prolonged intravaginal delivery and retention of elvitegravir. Biomaterials. 144, 144-154 (2017).

44 Ensign, L., Cone, R. \& Hanes, J. (Google Patents, 2017).

45 Lai, S. K., O'Hanlon, E. D., Man, S. T., Cone, R. \& Hanes, J. in 05AIChE: 2005 AIChE Annual Meeting and Fall Showcase.

46 Meng, J. et al. Tenofovir containing thiolated chitosan core/shell nanofibers: in vitro and in vivo evaluations. Mol Pharm. 13, 4129-4140 (2016).

47 Zamani, M., Prabhakaran, M. P. \& Ramakrishna, S. Advances in drug delivery via electrospun and electrosprayed nanomaterials. Int J Nanomedicine 8, 2997 (2013).

$48 \mathrm{Hu}, \mathrm{X}$. et al. Electrospinning of polymeric nanofibers for drug delivery applications. Journal of controlled release 185, 12-21 (2014).

49 Jain, K. K. Drug delivery systems. Vol. 437 (Springer Science \& Business Media, 2008).

50 Sharma, R. et al. Recent advances in polymeric electrospun nanofibers for drug delivery. Crit Rev Ther Drug Carrier Syst. 31 (2014).

51 Repanas, A., Andriopoulou, S. \& Glasmacher, B. The significance of electrospinning as a method to create fibrous scaffolds for biomedical engineering and drug delivery applications. J Drug Deliv Sci Technol 31, 137-146 (2016).

$52 \mathrm{Fu}, \mathrm{Y}$. \& Kao, W. J. Drug release kinetics and transport mechanisms of nondegradable and degradable polymeric delivery systems. Expert Opin Drug Deliv. 7, 429-444 (2010). 
Kim, T. G., Lee, D. S. \& Park, T. G. Controlled protein release from electrospun biodegradable fiber mesh composed of poly ( $\varepsilon$-caprolactone) and poly (ethylene oxide). Int J Pharm 338, 276-283 (2007).

54 Qi, H., Hu, P., Xu, J. \& Wang, A. Encapsulation of drug reservoirs in fibers by emulsion electrospinning: morphology characterization and preliminary release assessment. Biomacromolecules 7, 2327-2330 (2006).

55 Nair, L. S. \& Laurencin, C. T. Biodegradable polymers as biomaterials. Prog. Polym. Sci. 32, 762-798 (2007).

56 Liu, H., Leonas, K. K. \& Zhao, Y. Antimicrobial properties and release profile of ampicillin from electrospun poly ( $\varepsilon$-caprolactone) nanofiber yarns. J Eng Fiber Fabr 5, 10-19 (2010).

57 Yoshimoto, H., Shin, Y., Terai, H. \& Vacanti, J. A biodegradable nanofiber scaffold by electrospinning and its potential for bone tissue engineering. Biomaterials. 24, 2077-2082 (2003).

58 Luu, Y., Kim, K., Hsiao, B., Chu, B. \& Hadjiargyrou, M. Development of a nanostructured DNA delivery scaffold via electrospinning of PLGA and PLA-PEG block copolymers. J. Control. Release 89, 341-353 (2003).

59 Puppi, D. et al. Nano/microfibrous polymeric constructs loaded with bioactive agents and designed for tissue engineering applications: a review. J biomed mater res $B .102,1562-1579$ (2014).

60 Cipitria, A., Skelton, A., Dargaville, T., Dalton, P. \& Hutmacher, D. Design, fabrication and characterization of PCL electrospun scaffolds-a review. J. Mater. Chem. A 21, 9419-9453 (2011).

61 Uhrich, K. E., Cannizzaro, S. M., Langer, R. S. \& Shakesheff, K. M. Polymeric systems for controlled drug release. Chem. Rev. 99, 3181-3198 (1999).

62 Ulery, B. D., Nair, L. S. \& Laurencin, C. T. Biomedical applications of biodegradable polymers. Journal of polymer science Part B: polymer physics 49, 832-864 (2011).

63 Chen, D. W.-C. \& Liu, S.-J. Nanofibers used for delivery of antimicrobial agents. Nanomedicine 10, 1959-1971 (2015).

64 Blakney, A. K., Ball, C., Krogstad, E. A. \& Woodrow, K. A. Electrospun fibers for vaginal anti-HIV drug delivery. Antiviral Res 100, S9-S16 (2013).

65 Ramakrishna, S. et al. Electrospun nanofibers: solving global issues. Materials today 9, 40-50 (2006).

66 Göpferich, A. Mechanisms of polymer degradation and erosion. Biomaterials 17, 103-114 (1996).

67 Chou, S. F. \& Woodrow, K. A. Relationships between mechanical properties and drug release from electrospun fibers of PCL and PLGA blends. J Mech Behav Biomed 65, 724-733, doi:10.1016/j.jmbbm.2016.09.004 (2017).

68 von Burkersroda, F., Schedl, L. \& Göpferich, A. Why degradable polymers undergo surface erosion or bulk erosion. Biomaterials. 23, 4221-4231, doi:10.1016/s01429612(02)00170-9 (2002).

69 Doshi, J. \& Reneker, D. H. Electrospinning process and applications of electrospun fibers. J Electrostat. 35, 151-160 (1995).

70 Zeng, J. et al. Biodegradable electrospun fibers for drug delivery. J. Control. Release 92, 227-231 (2003).

71 Sill, T. J. \& von Recum, H. A. Electrospinning: applications in drug delivery and tissue engineering. Biomaterials. 29, 1989-2006 (2008).

72 Kenawy, E.-R., Abdel-Hay, F. I., El-Newehy, M. H. \& Wnek, G. E. in Nanomaterials: Risks and Benefits 247-263 (Springer, 2009). 
Ji, W. et al. Bioactive electrospun scaffolds delivering growth factors and genes for tissue engineering applications. Pharm Res 28, 1259-1272 (2011).

74 Pillay, V. et al. A review of the effect of processing variables on the fabrication of electrospun nanofibers for drug delivery applications. J Nanomater. 2013 (2013).

75 Xie, J., Li, X. \& Xia, Y. Putting electrospun nanofibers to work for biomedical research. Macromol. Rapid Commun. 29, 1775-1792 (2008).

76 Subbiah, T., Bhat, G., Tock, R., Parameswaran, S. \& Ramkumar, S. Electrospinning of nanofibers. J. Appl. Polym. Sci. 96, 557-569 (2005).

77 Hadjiargyrou, M. \& Chiu, J. B. Enhanced composite electrospun nanofiber scaffolds for use in drug delivery. Expert opinion on drug delivery 5, 1093-1106 (2008).

78 Zhang, Y., Lim, C. T., Ramakrishna, S. \& Huang, Z.-M. Recent development of polymer nanofibers for biomedical and biotechnological applications. Journal of Materials Science: Materials in Medicine 16, 933-946 (2005).

79 Verreck, G. et al. Incorporation of drugs in an amorphous state into electrospun nanofibers composed of a water-insoluble, nonbiodegradable polymer. Journal of controlled release 92, 349-360 (2003).

80 Han, D. \& Steckl, A. J. Triaxial electrospun nanofiber membranes for controlled dual release of functional molecules. ACS applied materials \& interfaces 5, 82418245 (2013).

81 Yarin, A. Coaxial electrospinning and emulsion electrospinning of core-shell fibers. Polymers for Advanced Technologies 22, 310-317 (2011).

$82 \mathrm{He}, \mathrm{C}$. L. et al. Coaxial electrospun poly (L-lactic acid) ultrafine fibers for sustained drug delivery. J Macromol Sci B 45, 515-524 (2006).

83 Lu, Y. et al. Coaxial electrospun fibers: applications in drug delivery and tissue engineering. Wiley Interdisciplinary Reviews: Nanomedicine and Nanobiotechnology 8, 654-677 (2016).

84 Yu, D. G. et al. Improving Polymer Nanofiber Quality Using a Modified Co-axial Electrospinning Process. Macromol. Rapid Commun. 32, 744-750 (2011).

85 Nezarati, R. M., Eifert, M. B. \& Cosgriff-Hernandez, E. Effects of humidity and solution viscosity on electrospun fiber morphology. Tissue Engineering Part C: Methods 19, 810-819 (2013).

86 Wang, J., Jákli, A. \& West, J. L. Morphology tuning of electrospun liquid crystal/polymer fibers. ChemPhysChem 17, 3080-3085 (2016).

87 Yang, J.-M., Zha, L.-S., Yu, D.-G. \& Liu, J. Coaxial electrospinning with acetic acid for preparing ferulic acid/zein composite fibers with improved drug release profiles. Colloids Surf B Biointerfaces. 102, 737-743 (2013).

88 Tang, C., Ozcam, A. E., Stout, B. \& Khan, S. A. Effect of pH on protein distribution in electrospun PVA/BSA composite nanofibers. Biomacromolecules 13, 12691278 (2012).

89 He, M., Jiang, H., Wang, R., Xie, Y. \& Zhao, C. Fabrication of metronidazole loaded poly $(\varepsilon$-caprolactone)/zein core/shell nanofiber membranes via coaxial electrospinning for guided tissue regeneration. J. Colloid Interface Sci. 490, 270278 (2017).

90 Wang, C., Yan, K.-W., Lin, Y.-D. \& Hsieh, P. C. Biodegradable core/shell fibers by coaxial electrospinning: processing, fiber characterization, and its application in sustained drug release. Macromolecules 43, 6389-6397 (2010).

91 Perrie, Y. \& Rades, T. FASTtrack Pharmaceutics: Drug Delivery and Targeting. (Pharmaceutical press, 2012).

92 Jiang, Y.-N., Mo, H.-Y. \& Yu, D. Electrospun drug-loaded core-sheath PVP/zein nanofibers for biphasic drug release. Vol. 438 (2012). 
93 Zhu, L., Liu, X., Du, L. \& Jin, Y. Preparation of asiaticoside-loaded coaxially electrospinning nanofibers and their effect on deep partial-thickness burn injury. Biomed Pharmacother 83, 33-40, doi:10.1016/j.biopha.2016.06.016 (2016).

94 Castillo-Ortega, M. et al. Preparation by coaxial electrospinning and characterization of membranes releasing (-) epicatechin as scaffold for tissue engineering. Materials Science and Engineering: C 46, 184-189 (2015).

95 Li, X.-Y., Li, Y.-C., Yu, D.-G., Liao, Y.-Z. \& Wang, X. Fast disintegrating quercetinloaded drug delivery systems fabricated using coaxial electrospinning. International journal of molecular sciences 14, 21647-21659 (2013).

$96 \mathrm{Yu}, \mathrm{D}$.-G. et al. Solid dispersions in the form of electrospun core-sheath nanofibers. International journal of nanomedicine 6, 3271 (2011).

97 Fu, L., Zhang, J. \& Yang, G. Present status and applications of bacterial cellulosebased materials for skin tissue repair. Carbohydrate Polymers 92, 1432-1442, doi:https://doi.org/10.1016/i.carbpol.2012.10.071 (2013).

$98 \mathrm{Yu}, \mathrm{D} . \mathrm{-G}$. et al. Nanofibers fabricated using triaxial electrospinning as zero order drug delivery systems. ACS applied materials \& interfaces 7, 18891-18897 (2015).

99 Nakielski, P. et al. Hydrogel nanofilaments via core-shell electrospinning. PloS one 10, e0129816 (2015).

100 Zhu, Y. J. \& Chen, F. pH-Responsive Drug-Delivery Systems. Chemistry-An Asian Journal 10, 284-305 (2015).

101 Yang, C. et al. Electrospun pH-sensitive core-shell polymer nanocomposites fabricated using a tri-axial process. Acta biomaterialia 35, 77-86 (2016).

102 Thakral, S., Thakral, N. K. \& Majumdar, D. K. Eudragit $\AA^{8}$ : a technology evaluation. Expert opinion on drug delivery 10, 131-149 (2013).

103 Yoshida, T., Lai, T. C., Kwon, G. S. \& Sako, K. pH-and ion-sensitive polymers for drug delivery. Expert opinion on drug delivery 10, 1497-1513 (2013).

104 Marrazzo, J. M. et al. Tenofovir-based preexposure prophylaxis for HIV infection among African women. The New England journal of medicine 372, 509-518, doi:10.1056/NEJMoa1402269 (2015).

105 Jin, M., Yu, D.-G., Geraldes, C. F., Williams, G. R. \& Bligh, S. A. Theranostic fibers for simultaneous imaging and drug delivery. Molecular pharmaceutics 13, $2457-$ 2465 (2016).

106 Jia, D., Gao, Y. \& Williams, G. R. Core/shell poly (ethylene oxide)/Eudragit fibers for site-specific release. International journal of pharmaceutics 523, 376-385 (2017).

107 Hua, D. et al. pH responsive polyurethane (core) and cellulose acetate phthalate (shell) electrospun fibers for intravaginal drug delivery. Carbohydr Polym 151, 1240-1244, doi:https://doi.org/10.1016/i.carbpol.2016.06.066 (2016).

108 Sang, Q., Li, H., Williams, G., Wu, H. \& Zhu, L.-M. Core-shell poly (lactide-co- $\varepsilon-$ caprolactone)-gelatin fiber scaffolds as $\mathrm{pH}$-sensitive drug delivery systems. Journal of biomaterials applications, 0885328217749962 (2018).

109 Han, D., Yu, X., Chai, Q., Ayres, N. \& Steckl, A. J. Stimuli-responsive selfimmolative polymer nanofiber membranes formed by coaxial electrospinning. ACS Appl. Mater. Interfaces 9, 11858-11865 (2017).

110 Ball, C., Chou, S.-F., Jiang, Y. \& Woodrow, K. A. Coaxially electrospun fiber-based microbicides facilitate broadly tunable release of maraviroc. Mater. Sci. Eng. C. 63, 117-124, doi:https://doi.org/10.1016/j.msec.2016.02.018 (2016).

111 Zhang, Y. et al. Coaxial electrospinning of (fluorescein isothiocyanate-conjugated bovine serum albumin)-encapsulated poly ( $\varepsilon$-caprolactone) nanofibers for sustained release. Biomacromolecules 7, 1049-1057 (2006). 
$112 \mathrm{Yu}, \mathrm{H}$. et al. Regulation of biphasic drug release behavior by graphene oxide in polyvinyl pyrrolidone/poly ( $\varepsilon$-caprolactone) core/sheath nanofiber mats. Colloids and Surfaces B: Biointerfaces 146, 63-69 (2016).

113 Oliveira, M. F. et al. Electrospun nanofibers of polyCD/PMAA polymers and their potential application as drug delivery system. Mater. Sci. Eng. C. 54, 252-261 (2015).

114 Sultanova, Z., Kaleli, G., Kabay, G. \& Mutlu, M. Controlled release of a hydrophilic drug from coaxially electrospun polycaprolactone nanofibers. Int $J$ Pharm 505, 133-138, doi:10.1016/j.ijpharm.2016.03.032 (2016).

115 Lv, Y. et al. Core-Sheath Nanofibers as Drug Delivery System for Thermoresponsive Controlled Release. J Pharm Sci 106, 1258-1265, doi:10.1016/j.xphs.2016.12.031 (2017).

116 Khalf, A. \& Madihally, S. V. Modeling the permeability of multiaxial electrospun poly $(\varepsilon$-caprolactone)-gelatin hybrid fibers for controlled doxycycline release. Mater. Sci. Eng. C. 76, 161-170 (2017).

117 Ranjbar-Mohammadi, M., Zamani, M., Prabhakaran, M. P., Bahrami, S. H. \& Ramakrishna, S. Electrospinning of PLGA/gum tragacanth nanofibers containing tetracycline hydrochloride for periodontal regeneration. Mater Sci Eng C Mater Biol Appl 58, 521-531, doi:10.1016/j.msec.2015.08.066 (2016).

118 Xie, Q. et al. Fabrication of core-shell PEI/pBMP2-PLGA electrospun scaffold for gene delivery to periodontal ligament stem cells. Stem cells international 2016 (2016).

119 Jiang, H., Hu, Y., Zhao, P., Li, Y. \& Zhu, K. Modulation of protein release from biodegradable core-shell structured fibers prepared by coaxial electrospinning. $J$ Biomed Mater Res B Appl Biomater 79, 50-57, doi:10.1002/jbm.b.30510 (2006).

120 Jiang, $H$. et al. A facile technique to prepare biodegradable coaxial electrospun nanofibers for controlled release of bioactive agents. J Control Release 108, 237243, doi:10.1016/j.jconrel.2005.08.006 (2005).

121 Yang, Y., Li, X., Qi, M., Zhou, S. \& Weng, J. Release pattern and structural integrity of lysozyme encapsulated in core-sheath structured poly (DL-lactide) ultrafine fibers prepared by emulsion electrospinning. European Journal of Pharmaceutics and Biopharmaceutics 69, 106-116 (2008).

$122 \mathrm{Ji}, \mathrm{W}$. et al. Fibrous scaffolds loaded with protein prepared by blend or coaxial electrospinning. Acta Biomater 6, 4199-4207, doi:10.1016/j.actbio.2010.05.025 (2010).

123 Saraf, A., Baggett, L. S., Raphael, R. M., Kasper, F. K. \& Mikos, A. G. Regulated non-viral gene delivery from coaxial electrospun fiber mesh scaffolds. J Control Release 143, 95-103, doi:10.1016/j.jconrel.2009.12.009 (2010).

$124 \mathrm{Hsu}, \mathrm{Y} . \mathrm{H}$. et al. Dual delivery of active antibactericidal agents and bone morphogenetic protein at sustainable high concentrations using biodegradable sheath-core-structured drug-eluting nanofibers. Int J Nanomedicine 11, 3927 (2016).

125 Aniagyei, S. E. et al. Evaluation of poly(lactic-co-glycolic acid) and poly(dl-lactideco- $\varepsilon$-caprolactone) electrospun fibers for the treatment of HSV-2 infection. Mater. Sci. Eng. C. 72, 238-251, doi:https://doi.org/10.1016/i.msec.2016.11.029 (2017).

126 Ball, C. \& Woodrow, K. A. Electrospun Solid Dispersions of Maraviroc for Rapid Intravaginal Preexposure Prophylaxis of HIV. Antimicrob. Agents Chemother. 58, 4855-4865, doi:10.1128/aac.02564-14 (2014).

127 Tyo, K. M. et al. Multipurpose tenofovir disoproxil fumarate electrospun fibers for the prevention of HIV-1 and HSV-2 infections in vitro. Int J Pharm 531, 118-133, doi:10.1016/j.jpharm.2017.08.061 (2017). 
128 Grooms, T. N. et al. Griffithsin-modified electrospun fibers as a delivery scaffold to prevent HIV infection. Antimicrobial agents and chemotherapy 60, 6518-6531 (2016).

129 Tyo, K. M. et al. pH-responsive delivery of Griffithsin from electrospun fibers. Eur J Pharm Biopharm, doi:10.1016/j.ejpb.2018.04.013 (2018).

130 Carson, D., Jiang, Y. \& Woodrow, K. A. Tunable Release of Multiclass Anti-HIV Drugs that are Water-Soluble and Loaded at High Drug Content in Polyester Blended Electrospun Fibers. Pharm Res 33, 125-136, doi:10.1007/s11095-0151769-0 (2016).

131 Liu, L. et al. Nano-on-micro fibrous extracellular matrices for scalable expansion of human ES/iPS cells. Biomaterials. 124, 47-54 (2017).

132 Huang, L.-Y., Branford-White, C., Shen, X.-X., Yu, D.-G. \& Zhu, L.-M. Timeengineeringed biphasic drug release by electrospun nanofiber meshes. Int $J$ Pharm 436, 88-96 (2012).

133 Meinel, A. J., Germershaus, O., Luhmann, T., Merkle, H. P. \& Meinel, L. Electrospun matrices for localized drug delivery: current technologies and selected biomedical applications. European Journal of Pharmaceutics and Biopharmaceutics 81, 1-13 (2012).

134 Blakney, A. K., Krogstad, E. A., Jiang, Y. H. \& Woodrow, K. A. Delivery of multipurpose prevention drug combinations from electrospun nanofibers using composite microarchitectures. Int J Nanomedicine 9, 2967 (2014).

135 Mehrotra, S. et al. Time controlled protein release from layer-by-layer assembled multilayer functionalized agarose hydrogels. Advanced func mater. 20, 247-258 (2010).

136 Pan, H., Li, L., Hu, L. \& Cui, X. Continuous aligned polymer fibers produced by a modified electrospinning method. Polymer 47, 4901-4904, doi:https://doi.org/10.1016/i.polymer.2006.05.012 (2006).

137 Shin, J.-W. et al. in 3rd Kuala Lumpur International Conference on Biomedical Engineering 2006. 692-695 (Springer).

138 Baker, B. M. et al. The potential to improve cell infiltration in composite fiberaligned electrospun scaffolds by the selective removal of sacrificial fibers. Biomaterials 29, 2348-2358, doi:10.1016/j.biomaterials.2008.01.032 (2008).

139 Tijing, L. D. et al. One-step fabrication of antibacterial (silver nanoparticles/poly(ethylene oxide)) - Polyurethane bicomponent hybrid nanofibrous mat by dual-spinneret electrospinning. Mater. Chem. Phys. 134, 557561, doi:DOI:101016/jmatchemphys201203037 (2012).

140 Wulkersdorfer, B. et al. Bimodal porous scaffolds by sequential electrospinning of poly (glycolic acid) with sucrose particles. International Journal of Polymer Science 2010 (2010).

141 Wan, A. C. \& Ying, J. Y. Nanomaterials for in situ cell delivery and tissue regeneration. Advanced drug delivery reviews 62, 731-740 (2010).

142 Dvir, T., Timko, B. P., Kohane, D. S. \& Langer, R. Nanotechnological strategies for engineering complex tissues. Nature Nanotechnology 6, 13 (2011).

143 Kharaziha, M., Fathi, M. \& Edris, H. Tunable cellular interactions and physical properties of nanofibrous PCL-forsterite: gelatin scaffold through sequential electrospinning. Composites Science and Technology 87, 182-188 (2013).

144 Tan, L., Hu, J. \& Zhao, H. Design of bilayered nanofibrous mats for wound dressing using an electrospinning technique. Materials Letters 156, 46-49 (2015).

145 Falde, E. J. et al. Layered superhydrophobic meshes for controlled drug release. J. Control. Release 214, 23-29, doi:10.1016/j.jconrel.2015.06.042 (2015). 
146 Sirc, J. et al. Controlled gentamicin release from multi-layered electrospun nanofibrous structures of various thicknesses. International journal of nanomedicine 7, 5315 (2012).

147 Mandal, B. B., Mann, J. K. \& Kundu, S. Silk fibroin/gelatin multilayered films as a model system for controlled drug release. European Journal of Pharmaceutical Sciences 37, 160-171 (2009).

148 Okuda, T., Tominaga, K. \& Kidoaki, S. Time-programmed dual release formulation by multilayered drug-loaded nanofiber meshes. J Control Release 143, 258-264, doi:10.1016/j.jconrel.2009.12.029 (2010).

149 Liu, S. et al. Use of asymmetric multilayer polylactide nanofiber mats in controlled release of drugs and prevention of liver cancer recurrence after surgery in mice. Nanomedicine: Nanotechnology, Biology and Medicine 11, 1047-1056 (2015).

150 Chunder, A., Sarkar, S., Yu, Y. \& Zhai, L. Fabrication of ultrathin polyelectrolyte fibers and their controlled release properties. Colloids and Surfaces B: Biointerfaces 58, 172-179 (2007).

151 Son, Y. J., Kim, W. J. \& Yoo, H. S. Therapeutic applications of electrospun nanofibers for drug delivery systems. Arch. Pharmacal Res. 37, 69-78 (2014).

152 Yoon, H. \& Kim, G. H. Layer-by-layered electrospun micro/nanofibrous mats for drug delivery system. Macromolecular Research 20, 402-406, doi:10.1007/s13233-012-0047-9 (2012).

153 Park, J. H., Kim, B. S., Yoo, Y. C., Khil, M. S. \& Kim, H. Y. Enhanced mechanical properties of multilayer nano-coated electrospun nylon 6 fibers via a layer-by-layer self-assembly. J. Appl. Polym. Sci. 107, 2211-2216 (2008).

154 Woodrow, K. A. et al. Intravaginal gene silencing using biodegradable polymer nanoparticles densely loaded with small-interfering RNA. Nature materials 8, 526533, doi:10.1038/nmat2444 (2009).

155 Ensign, L. M., Cone, R. \& Hanes, J. Nanoparticle-based drug delivery to the vagina: a review. $J$ Control Release 190, 500-514, doi:10.1016/j.jconrel.2014.04.033 (2014).

156 Ahmad, M. Z. et al. Metallic nanoparticles: technology overview \& drug delivery applications in oncology. Expert Opin Drug Deliv 7, 927-942, doi:10.1517/17425247.2010.498473 (2010).

157 Mody, V. V., Siwale, R., Singh, A. \& Mody, H. R. Introduction to metallic nanoparticles. J Pharm Bioallied Sci 2, 282-289, doi:10.4103/0975-7406.72127 (2010).

158 Singh, R. \& Lillard, J. W., Jr. Nanoparticle-based targeted drug delivery. Exp Mol Pathol. 86, 215-223, doi:10.1016/j.yexmp.2008.12.004 (2009).

159 Blanco, E., Shen, H. \& Ferrari, M. Principles of nanoparticle design for overcoming biological barriers to drug delivery. Nature biotechnology 33, 941-951 (2015).

160 Peer, D. et al. Nanocarriers as an emerging platform for cancer therapy. Nature nanotechnology 2, 751-760 (2007).

$161 \mathrm{Gu}$, J., Yang, S. \& Ho, E. A. Biodegradable film for the targeted delivery of siRNAloaded nanoparticles to vaginal immune cells. Mol Pharm. 12, 2889-2903 (2015).

162 Wang, Y. Y. et al. Addressing the PEG mucoadhesivity paradox to engineer nanoparticles that "slip" through the human mucus barrier. Angewandte Chemie International Edition 47, 9726-9729 (2008).

163 Wang, S., Zhao, Y., Shen, M. \& Shi, X. Electrospun hybrid nanofibers doped with nanoparticles or nanotubes for biomedical applications. Ther Deliv. 3, 1155-1169 (2012).

164 Chen, M. et al. Chitosan/siRNA nanoparticles encapsulated in PLGA nanofibers for siRNA delivery. ACS nano 6, 4835-4844 (2012). 
165 Sridhar, R. et al. Electrosprayed nanoparticles and electrospun nanofibers based on natural materials: applications in tissue regeneration, drug delivery and pharmaceuticals. Chem. Soc. Rev. 44, 790-814 (2015).

166 Mehrasa, M. et al. Incorporation of mesoporous silica nanoparticles into random electrospun PLGA and PLGA/gelatin nanofibrous scaffolds enhances mechanical and cell proliferation properties. Mater. Sci. Eng. C. 66, 25-32 (2016).

167 Song, B., Wu, C. \& Chang, J. Controllable delivery of hydrophilic and hydrophobic drugs from electrospun poly (lactic-co-glycolic acid)/mesoporous silica nanoparticles composite mats. J biomed mater res B. 100, 2178-2186 (2012).

168 Beck-Broichsitter, M. et al. Novel 'Nano in Nano'Composites for Sustained Drug Delivery: Biodegradable Nanoparticles Encapsulated into Nanofiber Non-Wovens. Macromolecular bioscience 10, 1527-1535 (2010).

169 Fathollahipour, S., Abouei Mehrizi, A., Ghaee, A. \& Koosha, M. Electrospinning of PVA/chitosan nanocomposite nanofibers containing gelatin nanoparticles as a dual drug delivery system. Journal of Biomedical Materials Research Part A 103, 3852-3862 (2015).

$170 \mathrm{Hu}$, J., Zeng, F., Wei, J., Chen, Y. \& Chen, Y. Novel controlled drug delivery system for multiple drugs based on electrospun nanofibers containing nanomicelles. Journal of Biomaterials Science, Polymer Edition 25, 257-268 (2014).

171 Wang, Y., Wang, B., Qiao, W. \& Yin, T. A novel controlled release drug delivery system for multiple drugs based on electrospun nanofibers containing nanoparticles. Journal of pharmaceutical sciences 99, 4805-4811 (2010).

$172 \mathrm{Li}$, L. et al. Controlled dual delivery of BMP-2 and dexamethasone by nanoparticleembedded electrospun nanofibers for the efficient repair of critical-sized rat calvarial defect. Biomaterials. 37, 218-229, doi:https://doi.org/10.1016/i.biomaterials.2014.10.015 (2015).

173 Ali, I. H., Khalil, I. A. \& El-Sherbiny, I. M. Single-Dose Electrospun Nanoparticlesin-Nanofibers Wound Dressings with Enhanced Epithelialization, Collagen Deposition, and Granulation Properties. ACS applied materials \& interfaces 8, 14453-14469 (2016).

174 Sun, X. et al. Rationally designed particle preloading method to improve protein delivery performance of electrospun polyester nanofibers. International journal of pharmaceutics 512, 204-212 (2016).

175 Vakilian, S. et al. Structural stability and sustained release of protein from a multilayer nanofiber/nanoparticle composite. International journal of biological macromolecules 75, 248-257 (2015).

176 Nie, H. \& Wang, C.-H. Fabrication and characterization of PLGA/HAp composite scaffolds for delivery of BMP-2 plasmid DNA. Journal of Controlled Release 120, 111-121, doi:https://doi.org/10.1016/j.jconrel.2007.03.018 (2007).

177 Cui, W., Zhou, Y. \& Chang, J. Electrospun nanofibrous materials for tissue engineering and drug delivery. Sci. Technol. Adv. Mater 11, 014108 (2010).

178 Shao, W. et al. Coaxial electrospun aligned tussah silk fibroin nanostructured fiber scaffolds embedded with hydroxyapatite-tussah silk fibroin nanoparticles for bone tissue engineering. Materials Science and Engineering: C 58, 342-351, doi:https://doi.org/10.1016/j.msec.2015.08.046 (2016).

179 Weissleder, R., Kelly, K., Sun, E. Y., Shtatland, T. \& Josephson, L. Cell-specific targeting of nanoparticles by multivalent attachment of small molecules. Nature biotechnology 23, 1418 (2005).

180 Kohler, N., Fryxell, G. E. \& Zhang, M. A bifunctional poly (ethylene glycol) silane immobilized on metallic oxide-based nanoparticles for conjugation with cell targeting agents. JACS 126, 7206-7211 (2004). 
181 Yao, L., Lin, Y. \& Watkins, J. J. Ultrahigh loading of nanoparticles into ordered block copolymer composites. Macromolecules 47, 1844-1849 (2014).

182 Zhu, J. et al. Electrospun polyimide nanocomposite fibers reinforced with coreshell Fe-FeO nanoparticles. J. Phys. Chem. C 114, 8844-8850 (2010).

183 Delany-Moretlwe, S. et al. Tenofovir $1 \%$ vaginal gel for prevention of HIV-1 infection in women in South Africa (FACTS-001): a phase 3, randomised, doubleblind, placebo-controlled trial. The Lancet Infectious Diseases 18, 1241-1250, doi:https://doi.org/10.1016/S1473-3099(18)30428-6 (2018).

184 Skoler-Karpoff, S. et al. Efficacy of Carraguard for prevention of HIV infection in women in South Africa: a randomised, double-blind, placebo-controlled trial. The Lancet 372, 1977-1987, doi:https://doi.org/10.1016/S0140-6736(08)61842-5 (2008).

185 Thurman, A. R., Clark, M. R. \& Doncel, G. F. Multipurpose prevention technologies: biomedical tools to prevent HIV-1, HSV-2, and unintended pregnancies. Infect Dis Obstet Gynecol 2011, 1-10, doi:10.1155/2011/429403 (2011).

186 Blakney, A. K., Simonovsky, F. I., Suydam, I. T., Ratner, B. D. \& Woodrow, K. A. Rapidly Biodegrading PLGA-Polyurethane Fibers for Sustained Release of Physicochemically Diverse Drugs. ACS Biomaterials Science \& Engineering 2, 1595-1607, doi:10.1021/acsbiomaterials.6b00346 (2016).

187 Halwes, M. E., Tyo, K. M., Steinbach-Rankins, J. M. \& Frieboes, H. B. Computational Modeling of Antiviral Drug Diffusion from Poly(lactic- co-glycolicacid) Fibers and Multicompartment Pharmacokinetics for Application to the Female Reproductive Tract. Mol Pharm 15, 1534-1547, doi:10.1021/acs.molpharmaceut.7b01089 (2018).

188 Moss, J. A. et al. Pharmacokinetics of a Multipurpose Pod-Intravaginal Ring Simultaneously Delivering Five Drugs in an Ovine Model. Antimicrob Agents Ch 57, 3994, doi:10.1128/AAC.00547-13 (2013).

189 Smith, J. M. et al. Novel multipurpose pod-intravaginal ring for the prevention of HIV, HSV, and unintended pregnancy: Pharmacokinetic evaluation in a macaque model. PloS one 12, e0185946-e0185946, doi:10.1371/journal.pone.0185946 (2017).

190 Morrow, R. J. et al. Sustained release of proteins from a modified vaginal ring device. European journal of pharmaceutics and biopharmaceutics : official journal of Arbeitsgemeinschaft fur Pharmazeutische Verfahrenstechnik e.V 77, 3-10, doi:10.1016/j.ejpb.2010.10.010 (2011).

191 Han, Y. A., Singh, M. \& Saxena, B. B. Development of vaginal rings for sustained release of nonhormonal contraceptives and anti-HIV agents. Contraception 76, 132-138, doi:https://doi.org/10.1016/..contraception.2007.04.006 (2007).

192 Malcolm, R. K. et al. Sustained Release of the CCR5 Inhibitors CMPD167 and Maraviroc from Vaginal Rings in Rhesus Macaques. Antimicrob Agents Ch 56, 2251, doi:10.1128/AAC.05810-11 (2012).

193 Johnson, T. J., Gupta, K. M., Fabian, J., Albright, T. H. \& Kiser, P. F. Segmented polyurethane intravaginal rings for the sustained combined delivery of antiretroviral agents dapivirine and tenofovir. European Journal of Pharmaceutical Sciences 39, 203-212, doi:https://doi.org/10.1016/i.ejps.2009.11.007 (2010).

194 Woolfson, A. D., Toner, C. F., Malcolm, R. K., Morrow, R. J. \& McCullagh, S. D. Long-term, controlled release of the HIV microbicide TMC120 from silicone elastomer vaginal rings. Journal of Antimicrobial Chemotherapy 56, 954-956, doi:10.1093/jac/dki326 (2005). 
195 Baum, M. M. et al. An Intravaginal Ring for the Simultaneous Delivery of Multiple Drugs. Journal of Pharmaceutical Sciences 101, 2833-2843, doi:https://doi.org/10.1002/jps.23208 (2012).

196 Johnson, T. J. et al. A 90-Day Tenofovir Reservoir Intravaginal Ring for Mucosal HIV Prophylaxis. Antimicrob Agents Ch 56, 6272, doi:10.1128/AAC.01431-12 (2012).

197 Blakney, A. K., Little, A. B., Jiang, Y. \& Woodrow, K. A. In vitro-ex vivo correlations between a cell-laden hydrogel and mucosal tissue for screening composite delivery systems. Drug delivery 24, 582-590 (2017).

198 Rohan, L. C. et al. In vitro and ex vivo testing of tenofovir shows it is effective as an HIV-1 microbicide. PLoS One 5, e9310 (2010).

199 Patton, D. et al. Preclinical safety assessments of UC781 anti-human immunodeficiency virus topical microbicide formulations. Antimicrob Agents Ch 51, 1608-1615 (2007).

200 Robinson, J. A. et al. Comparison of the Pharmacokinetics and Pharmacodynamics of Single-Dose Tenofovir Vaginal Film and Gel Formulation (FAME 05). J Acquir Immune Defic Syndr 77, 175-182, doi:10.1097/QAI.0000000000001587 (2018).

201 Hu, M., Zhou, T., Dezzutti, C. S. \& Rohan, L. C. The effect of commonly used excipients on the epithelial integrity of human cervicovaginal tissue. AIDS research and human retroviruses 32, 992-1004 (2016).

202 Merbah, M. et al. Cervico-vaginal tissue ex vivo as a model to study early events in HIV-1 infection. American Journal of Reproductive Immunology 65, 268-278 (2011).

203 Ayehunie, S. et al. Organotypic human vaginal-ectocervical tissue model for irritation studies of spermicides, microbicides, and feminine-care products. Toxicology in Vitro 20, 689-698 (2006).

204 Ayehunie, S. et al. Development of an in vitro alternative assay method for vaginal irritation. Toxicology 279, 130-138 (2011).

205 Łaniewski, P., Gomez, A., Hire, G., So, M. \& Herbst-Kralovetz, M. M. Human threedimensional endometrial epithelial cell model to study host interactions with vaginal bacteria and Neisseria gonorrhoeae. Infection and immunity 85, e01049-01016 (2017).

206 Doncel, G. F. \& Clark, M. R. Preclinical evaluation of anti-HIV microbicide products: New models and biomarkers. Antiviral Res 88 Suppl 1, S10-18, doi:10.1016/j.antiviral.2010.09.018 (2010).

207 Huang, C. et al. Electrospun cellulose acetate phthalate fibers for semen induced anti-HIV vaginal drug delivery. Biomaterials 33, 962-969, doi:https://doi.org/10.1016/i.biomaterials.2011.10.004 (2012).

208 Ball, C., Krogstad, E., Chaowanachan, T. \& Woodrow, K. A. Drug-eluting fibers for HIV-1 inhibition and contraception. PloS one 7, e49792-e49792, doi:10.1371/journal.pone.0049792 (2012).

209 Krogstad, E. A. \& Woodrow, K. A. Manufacturing scale-up of electrospun poly(vinyl alcohol) fibers containing tenofovir for vaginal drug delivery. International Journal of Pharmaceutics 475, 282-291, doi:https://doi.org/10.1016/j.ijpharm.2014.08.039 (2014).

210 Jiang, J. et al. Mussel-inspired protein-mediated surface functionalization of electrospun nanofibers for $\mathrm{pH}$-responsive drug delivery. Acta Biomaterialia 10, 1324-1332, doi:https://doi.org/10.1016/..actbio.2013.11.012 (2014).

211 Sun, X.-Z., Williams, G. R., Hou, X.-X. \& Zhu, L.-M. Electrospun curcumin-loaded fibers with potential biomedical applications. Carbohydr Polym 94, 147-153 (2013). 
212 Berg, M. C., Zhai, L., Cohen, R. E. \& Rubner, M. F. Controlled drug release from porous polyelectrolyte multilayers. Biomacromolecules 7, 357-364 (2006).

213 Cohen, M. S. et al. Antiretroviral therapy for the prevention of HIV-1 transmission. New England Journal of Medicine 375, 830-839 (2016).

214 Rupp, R., Rosenthal, S. L. \& Stanberry, L. R. VivaGel( ${ }^{\mathrm{TM}}$ ) (SPL7013 Gel): A candidate dendrimer - microbicide for the prevention of HIV and HSV infection. International Journal of Nanomedicine 2, 561-566 (2007).

215 Prokofjeva, M. M., Kochetkov, S. N. \& Prassolov, V. S. Therapy of HIV Infection: Current Approaches and Prospects. Acta naturae 8, 23-32 (2016).

216 Broder, S. The development of antiretroviral therapy and its impact on the HIV1/AIDS pandemic. Antiviral research 85, 1-18 (2010).

217 Arts, E. J. \& Hazuda, D. J. HIV-1 antiretroviral drug therapy. Cold Spring Harbor perspectives in medicine 2, a007161 (2012).

218 Hicks, C. \& Gulick, R. M. Raltegravir: The First HIV Type 1 Integrase Inhibitor. Clinical Infectious Diseases 48, 931-939, doi:10.1086/597290 (2009).

219 Mamo, T. et al. Emerging nanotechnology approaches for HIV/AIDS treatment and prevention. Nanomedicine 5, 269-285 (2010).

220 Richman, D. D. et al. The toxicity of azidothymidine (AZT) in the treatment of patients with AIDS and AIDS-related complex. New England Journal of Medicine 317, 192-197 (1987).

221 Sheldon, J. et al. Selection of hepatitis B virus polymerase mutations in HIVcoinfected patients treated with tenofovir. Antiviral therapy 10, 727 (2005).

222 Ferir, G., Palmer, K. E. \& Schols, D. Synergistic activity profile of griffithsin in combination with tenofovir, maraviroc and enfuvirtide against HIV-1 clade C. Virology 417, 253-258, doi:10.1016/j.virol.2011.07.004 (2011).

223 Chupradit, K. et al. Current peptide and protein candidates challenging HIV therapy beyond the vaccine era. Viruses 9, 281 (2017).

224 Mitchell, C. A., Ramessar, K. \& O'Keefe, B. R. Antiviral lectins: Selective inhibitors of viral entry. Antiviral research 142, 37-54 (2017).

225 Baker, M. P. \& Carr, F. J. Pre-clinical considerations in the assessment of immunogenicity for protein therapeutics. Current drug safety 5, 308-313 (2010).

226 Mori, T. et al. Isolation and characterization of griffithsin, a novel HIV-inactivating protein, from the red alga Griffithsia sp. J Biol Chem 280, 9345-9353, doi:10.1074/jbc.M411122200 (2005).

227 Emau, P. et al. Griffithsin, a potent HIV entry inhibitor, is an excellent candidate for anti-HIV microbicide. Journal of medical primatology 36, 244-253 (2007).

228 O'Keefe, B. R. et al. Broad-spectrum in vitro activity and in vivo efficacy of the antiviral protein griffithsin against emerging viruses of the family Coronaviridae. $J$ Virol 84, 2511-2521, doi:10.1128/JVI.02322-09 (2010).

229 O'Keefe, B. R. et al. Scaleable manufacture of HIV-1 entry inhibitor griffithsin and validation of its safety and efficacy as a topical microbicide component. Proc Natl Acad Sci U S A 106, 6099-6104, doi:10.1073/pnas.0901506106 (2009).

230 Barton, C. et al. Activity of and effect of subcutaneous treatment with the broadspectrum antiviral lectin griffithsin in two laboratory rodent models. Antimicrob Agents Chemother 58, 120-127, doi:10.1128/AAC.01407-13 (2014).

231 Kouokam, J. C. et al. Investigation of griffithsin's interactions with human cells confirms its outstanding safety and efficacy profile as a microbicide candidate. PLoS One 6, e22635, doi:10.1371/journal.pone.0022635 (2011).

232 Günaydın, G. et al. Impact of Q-Griffithsin anti-HIV microbicide gel in non-human primates: In situ analyses of epithelial and immune cell markers in rectal mucosa. Scientific reports 9, 1-12 (2019). 
233 Corman, J. M. et al. Stability of plasmid and viral banks supporting the cGMP manufacture of Q-Griffithsin from a TMV-based viral vector. Journal of biotechnology 320, 74-76 (2020).

234 Jiang, Y. et al. Nanoparticle-based ARV drug combinations for synergistic inhibition of cell-free and cell-cell HIV transmission. Molecular pharmaceutics 12, 4363-4374 (2015).

235 Feng, J. Y. et al. The triple combination of tenofovir, emtricitabine and efavirenz shows synergistic anti-HIV-1 activity in vitro: a mechanism of action study. Retrovirology 6, 44 (2009).

236 Choopanya, K. et al. Antiretroviral prophylaxis for HIV infection in injecting drug users in Bangkok, Thailand (the Bangkok Tenofovir Study): a randomised, doubleblind, placebo-controlled phase 3 trial. The Lancet 381, 2083-2090 (2013).

237 Grant, R. M. et al. Preexposure chemoprophylaxis for HIV prevention in men who have sex with men. New England Journal of Medicine 363, 2587-2599 (2010).

238 Marrazzo, J. M. et al. Tenofovir-based preexposure prophylaxis for HIV infection among African women. New England Journal of Medicine 372, 509-518 (2015).

239 Thigpen, M. C. et al. Antiretroviral preexposure prophylaxis for heterosexual HIV transmission in Botswana. New England Journal of Medicine 367, 423-434 (2012).

240 Van Damme, L. et al. Preexposure prophylaxis for HIV infection among African women. New England Journal of Medicine 367, 411-422 (2012).

241 Haberer, J. E. Current Concepts for PrEP Adherence: In The PrEP revolution; from clinical trials to routine practice. Current opinion in HIV and AIDS 11, 10 (2016).

242 Rohan, L. C. \& Sassi, A. B. Vaginal Drug Delivery Systems for HIV Prevention. The AAPS Journal 11, 78, doi:10.1208/s12248-009-9082-7 (2009).

243 Guthrie, K. M. et al. The promise of intravaginal rings for prevention: user perceptions of biomechanical properties and implications for prevention product development. PLoS One 10, e0145642 (2015).

244 Emau, P. et al. Griffithsin, a potent HIV entry inhibitor, is an excellent candidate for anti-HIV microbicide. J Med Primatol 36, 244-253, doi:10.1111/j.16000684.2007.00242.x (2007).

245 Nixon, B. et al. Griffithsin protects mice from genital herpes by preventing cell-tocell spread. J Virol 87, 6257-6269, doi:10.1128/JVI.00012-13 (2013).

246 Steinbach, J. M. Protein and oligonucleotide delivery systems for vaginal microbicides against viral STls. Cellular and molecular life sciences : CMLS 72, 469-503, doi:10.1007/s00018-014-1756-3 (2015).

247 Steinbach, J. M., Seo, Y. E. \& Saltzman, W. M. Cell penetrating peptide-modified poly (lactic-co-glycolic acid) nanoparticles with enhanced cell internalization. Acta Biomater, doi:10.1016/j.actbio.2015.11.029 (2015).

248 Steinbach, J. M., Weller, C. E., Booth, C. J. \& Saltzman, W. M. Polymer nanoparticles encapsulating siRNA for treatment of HSV-2 genital infection. $J$ Control Release 162, 102-110, doi:10.1016/j.jconrel.2012.06.008 (2012).

249 Aniagyei, S. E. et al. Evaluation of poly(lactic-co-glycolic acid) and poly(dl-lactideco-epsilon-caprolactone) electrospun fibers for the treatment of HSV-2 infection. Mater Sci Eng C Mater Biol Appl 72, 238-251, doi:10.1016/j.msec.2016.11.029 (2017).

250 Ball, C., Krogstad, E., Chaowanachan, T. \& Woodrow, K. A. Drug-eluting fibers for HIV-1 inhibition and contraception. PLoS One 7, e49792, doi:10.1371/journal.pone.0049792 (2012).

251 Blakney, A. K., Ball, C., Krogstad, E. A. \& Woodrow, K. A. Electrospun fibers for vaginal anti-HIV drug delivery. Antiviral Res 100 Suppl, S9-16, doi:10.1016/j.antiviral.2013.09.022 (2013). 
252 Duan, J. H. \& Steinbach-Rankins, J. M. Adaptable Griffithsin Delivery from Polymer Blend Nanoparticles and Electrospun Fibers. Aids Research and Human Retroviruses 32, 218-218 (2016).

253 Huang, C. et al. Stimuli-responsive electrospun fibers and their applications. Chemical Society reviews 40, 2417-2434, doi:10.1039/c0cs00181c (2011).

254 Huang, C. et al. Electrospun cellulose acetate phthalate fibers for semen induced anti-HIV vaginal drug delivery. Biomaterials 33, 962-969, doi:10.1016/j.biomaterials.2011.10.004 (2012).

255 Mallipeddi, R. \& Rohan, L. C. Nanoparticle-based vaginal drug delivery systems for HIV prevention. Expert opinion on drug delivery 7, 37-48, doi:10.1517/17425240903338055 (2010).

256 Tyo, K. M. \& Steinbach-Rankins, J. M. Electrospun Polymer Nanofibers for Longterm Protection against HIV and HSV-2. Aids Research and Human Retroviruses 32, 223-223 (2016).

257 Wheeler, L. A. et al. Inhibition of HIV transmission in human cervicovaginal explants and humanized mice using CD4 aptamer-siRNA chimeras. The Journal of clinical investigation 121, 2401-2412, doi:10.1172/JCl45876 (2011).

258 Woodrow, K. A. et al. Intravaginal gene silencing using biodegradable polymer nanoparticles densely loaded with small-interfering RNA. Nature materials 8, 526533, doi:10.1038/nmat2444 (2009).

$259 \mathrm{Wu}, \mathrm{Y}$. et al. Durable protection from Herpes Simplex Virus-2 transmission following intravaginal application of siRNAs targeting both a viral and host gene. Cell host \& microbe 5, 84-94 (2009).

260 Zamani, M., Prabhakaran, M. P. \& Ramakrishna, S. Advances in drug delivery via electrospun and electrosprayed nanomaterials. Int J Nanomedicine 8, 2997-3017, doi:10.2147/IJN.S43575 (2013).

261 Krogstad, E. A. \& Woodrow, K. A. Manufacturing scale-up of electrospun poly(vinyl alcohol) fibers containing tenofovir for vaginal drug delivery. International journal of pharmaceutics 475, 282-291, doi:10.1016/j.ijpharm.2014.08.039 (2014).

262 Chaowanachan, T., Krogstad, E., Ball, C. \& Woodrow, K. A. Drug synergy of tenofovir and nanoparticle-based antiretrovirals for HIV prophylaxis. PLoS One 8, e61416, doi:10.1371/journal.pone.0061416 (2013).

263 Blakney, A. K., Krogstad, E. A., Jiang, Y. H. \& Woodrow, K. A. Delivery of multipurpose prevention drug combinations from electrospun nanofibers using composite microarchitectures. Int J Nanomedicine 9, 2967-2978, doi:10.2147/IJN.S61664 (2014).

264 Ball, C. \& Woodrow, K. A. Electrospun solid dispersions of Maraviroc for rapid intravaginal preexposure prophylaxis of HIV. Antimicrobial agents and chemotherapy 58, 4855-4865, doi:10.1128/AAC.02564-14 (2014).

265 Ball, C., Chou, S. F., Jiang, Y. \& Woodrow, K. A. Coaxially electrospun fiber-based microbicides facilitate broadly tunable release of maraviroc. Mater Sci Eng C Mater Biol App/ 63, 117-124, doi:10.1016/j.msec.2016.02.018 (2016).

266 Zhang, T., Sturgis, T. F. \& Youan, B.-B. C. pH-responsive nanoparticles releasing tenofovir intended for the prevention of HIV transmission. European Journal of Pharmaceutics and Biopharmaceutics 79, 526-536 (2011).

267 Machado, A. et al. Development and in vivo safety assessment of tenofovir-loaded nanoparticles-in-film as a novel vaginal microbicide delivery system. Acta biomaterialia 44, 332-340 (2016).

268 Ensign, L. M., Cone, R. \& Hanes, J. Nanoparticle-based drug delivery to the vagina: a review. Journal of Controlled Release 190, 500-514 (2014). 
269 Ensign, L. M. et al. Mucus-penetrating nanoparticles for vaginal drug delivery protect against herpes simplex virus. Science translational medicine 4, 138ra179138ra179 (2012).

270 Ensign, L. M. et al. Mucus-Penetrating Nanoparticles for Vaginal Drug Delivery Protect Against Herpes Simplex Virus. Science translational medicine 4, 10.1126/scitranslmed.3003453, doi:10.1126/scitranslmed.3003453 (2012).

271 das Neves, J. et al. Biodistribution and pharmacokinetics of dapivirine-loaded nanoparticles after vaginal delivery in mice. Pharmaceutical research 31, 18341845 (2014).

272 Hua, D. et al. pH responsive polyurethane (core) and cellulose acetate phthalate (shell) electrospun fibers for intravaginal drug delivery. Carbohydrate Polymers 151, 1240-1244, doi:https://doi.org/10.1016/i.carbpol.2016.06.066 (2016).

273 Tyo, K. M. et al. Sustained-release Griffithsin nanoparticle-fiber composites against HIV-1 and HSV-2 infections. Journal of Controlled Release 321, 84-99 (2020).

274 Elsabahy, M. \& Wooley, K. L. Design of polymeric nanoparticles for biomedical delivery applications. Chemical Society reviews 41, 2545-2561, doi:10.1039/c2cs15327k (2012).

275 Panyam, J. \& Labhasetwar, V. Biodegradable nanoparticles for drug and gene delivery to cells and tissue. Advanced drug delivery reviews 55, 329-347 (2003).

276 GeunHyung, K., Hyeon, Y. \& YunKyung, P. Drug release from various thicknesses of layered mats consisting of electrospun polycaprolactone and polyethylene oxide micro/nanofibers. Applied Physics A: Materials Science \& Processing 100, 11971204, doi:10.1007/s00339-010-5785-y (2010).

277 Wang, Y.-f., Guo, H.-f. \& Ying, D.-j. Multilayer scaffold of electrospun PLA-PCLcollagen nanofibers as a dural substitute. J Biomed Mater Res B Appl Biomater 101, 1359-1366, doi:10.1002/jbm.b.32953 (2013).

278 Liu, L. et al. Nano-on-micro fibrous extracellular matrices for scalable expansion of human ES/iPS cells. Biomaterials 124, 47-54 (2017).

279 Okuda, T., Tominaga, K. \& Kidoaki, S. Time-programmed dual release formulation by multilayered drug-loaded nanofiber meshes. Journal of Controlled Release 143, 258-264 (2010).

280 Kidoaki, S., Kwon, I. K. \& Matsuda, T. Mesoscopic spatial designs of nano- and microfiber meshes for tissue-engineering matrix and scaffold based on newly devised multilayering and mixing electrospinning techniques. Biomaterials 26, 3746 (2005).

281 Westling, K., Pettersson, K., Kaldma, A. \& Navér, L. Rapid decline in HIV viral load when introducing raltegravir-containing antiretroviral treatment late in pregnancy. AIDS patient care and STDs 26, 714-717 (2012).

282 Chou, T.-C. Drug combination studies and their synergy quantification using the Chou-Talalay method. Cancer research 70, 440-446 (2010).

283 Houdaihed, L., Evans, J. C. \& Allen, C. Codelivery of paclitaxel and everolimus at the optimal synergistic ratio: a promising solution for the treatment of breast cancer. Molecular pharmaceutics 15, 3672-3681 (2018).

284 Chou, T.-C. \& Talalay, P. Quantitative analysis of dose-effect relationships: the combined effects of multiple drugs or enzyme inhibitors. Advances in enzyme regulation 22, 27-55 (1984).

285 Schader, S. M., Colby-Germinario, S. P., Schachter, J. R., Xu, H. \& Wainberg, M. A. Synergy against drug-resistant HIV-1 with the microbicide antiretrovirals, dapivirine and tenofovir, in combination. Aids 25, 1585-1594 (2011). 
286 Yang, $\mathrm{H}$. et al. Design of poly (lactic-co-glycolic acid)(PLGA) nanoparticles for vaginal co-delivery of griffithsin and dapivirine and their synergistic effect for HIV prophylaxis. Pharmaceutics 11, 184 (2019).

287 Date, A. A. et al. Development and evaluation of a thermosensitive vaginal gel containing raltegravir+ efavirenz loaded nanoparticles for HIV prophylaxis. Antiviral research 96, 430-436 (2012).

288 Chen, B. A. et al. Phase 1 safety, pharmacokinetics, and pharmacodynamics of dapivirine and maraviroc vaginal rings: a double-blind randomized trial. Journal of acquired immune deficiency syndromes (1999) 70, 242 (2015).

289 Bani-Sadr, F., Palmer, P., Scieux, C. \& Molina, J. Ninety-Six-Week Efficacy of Combination Therapy with Lamivudine and Tenofovir in Patients Coinfected with HIV-1 and Wild-Type Hepatitis B Virus. Clinical infectious diseases 39, 1062-1064 (2004).

290 Lennox, J. L. et al. Safety and efficacy of raltegravir-based versus efavirenz-based combination therapy in treatment-naive patients with HIV-1 infection: a multicentre, double-blind randomised controlled trial. The Lancet 374, 796-806 (2009).

291 Dezzutti, C. S. et al. Safety and efficacy of tenofovir/IQP-0528 combination gelsa dual compartment microbicide for HIV-1 prevention. Antiviral research 96, 221225 (2012).

292 Steinbach, J. M., Weller, C. E., Booth, C. J. \& Saltzman, W. M. Polymer nanoparticles encapsulating siRNA for treatment of HSV-2 genital infection. Journal of Controlled Release 162, 102-110, doi:https://doi.org/10.1016/j.jconrel.2012.06.008 (2012).

293 Pagels, R. F. \& Prud'Homme, R. K. Polymeric nanoparticles and microparticles for the delivery of peptides, biologics, and soluble therapeutics. Journal of Controlled Release 219, 519-535 (2015).

294 Shailender, J., Ravi, P. R., Saha, P., Dalvi, A. \& Myneni, S. Tenofovir disoproxil fumarate loaded PLGA nanoparticles for enhanced oral absorption: Effect of experimental variables and in vitro, ex vivo and in vivo evaluation. Colloids and Surfaces B: Biointerfaces 158, 610-619 (2017).

295 das Neves, J. \& Sarmento, B. Precise engineering of dapivirine-loaded nanoparticles for the development of anti-HIV vaginal microbicides. Acta biomaterialia 18, 77-87 (2015).

296 Jäger, A. et al. Structural changes on polymeric nanoparticles induced by hydrophobic drug entrapment. Colloids and Surfaces A: Physicochemical and Engineering Aspects 538, 238-249 (2018).

297 Budhian, A., Siegel, S. J. \& Winey, K. I. Haloperidol-loaded PLGA nanoparticles: systematic study of particle size and drug content. International journal of pharmaceutics 336, 367-375 (2007).

298 Fletcher, P. et al. Inhibition of HIV-1 infection by the candidate microbicide, dapivirine, a non-nucleoside reverse transcriptase inhibitor. Antimicrob Agents Ch (2008).

299 Tomoda, K. et al. Enhanced transdermal delivery of indomethacin-loaded PLGA nanoparticles by iontophoresis. Colloids and Surfaces B: Biointerfaces 88, 706710 (2011).

300 Platt, L. et al. Prevalence and burden of HCV co-infection in people living with HIV: a global systematic review and meta-analysis. The Lancet infectious diseases 16, 797-808 (2016).

301 Chapman, T. M., McGavin, J. K. \& Noble, S. Tenofovir disoproxil fumarate. Drugs 63, 1597-1608 (2003). 
302 Evering, T. H. \& Markowitz, M. Raltegravir: an integrase inhibitor for HIV-1. Expert opinion on investigational drugs 17, 413-422 (2008).

303 Arora, R. Molecular mechanism of HIV-1 integrase inhibition by Raltegravir proposed by using of molecular modeling approaches, (2012).

304 Nuttall, J. P. et al. Concentrations of dapivirine in the rhesus macaque and rabbit following once daily intravaginal administration of a gel formulation of [14C] dapivirine for 7 days. Antimicrob Agents Ch 52, 909-914 (2008).

305 Meng, J., Sturgis, T. F. \& Youan, B.-B. C. Engineering tenofovir loaded chitosan nanoparticles to maximize microbicide mucoadhesion. European Journal of Pharmaceutical Sciences 44, 57-67 (2011).

306 Alukda, D., Sturgis, T. \& Youan, B. B. C. Formulation of tenofovir-loaded functionalized solid lipid nanoparticles intended for HIV prevention. Journal of pharmaceutical sciences 100, 3345-3356 (2011).

307 Joshi, G., Kumar, A. \& Sawant, K. Bioavailability enhancement, Caco-2 cells uptake and intestinal transport of orally administered lopinavir-loaded PLGA nanoparticles. Drug delivery 23, 3492-3504 (2016).

308 Patel, S., Lavasanifar, A. \& Choi, P. Application of molecular dynamics simulation to predict the compatability between water-insoluble drugs and self-associating poly (ethylene oxide)-b-poly ( $\varepsilon$-caprolactone) block copolymers. Biomacromolecules 9, 3014-3023 (2008).

309 Patel, S. K., Lavasanifar, A. \& Choi, P. Roles of nonpolar and polar intermolecular interactions in the improvement of the drug loading capacity of PEO-b-PCL with increasing PCL content for two hydrophobic cucurbitacin drugs. Biomacromolecules 10, 2584-2591 (2009).

310 Patel, S. K., Lavasanifar, A. \& Choi, P. Molecular dynamics study of the encapsulation capability of a PCL-PEO based block copolymer for hydrophobic drugs with different spatial distributions of hydrogen bond donors and acceptors. Biomaterials 31, 1780-1786 (2010).

311 Kasimova, A. O. et al. Validation of a novel molecular dynamics simulation approach for lipophilic drug incorporation into polymer micelles. The Journal of Physical Chemistry B 116, 4338-4345 (2012).

312 Chonco, L. et al. Carbosilane dendrimer nanotechnology outlines of the broad HIV blocker profile. Journal of controlled release 161, 949-958 (2012).

313 Costache, A. D., Sheihet, L., Zaveri, K., Knight, D. D. \& Kohn, J. Polymer- drug interactions in tyrosine-derived triblock copolymer nanospheres: a computational modeling approach. Molecular pharmaceutics 6, 1620-1627 (2009).

314 Sun, H., Ren, P. \& Fried, J. The COMPASS force field: parameterization and validation for phosphazenes. Computational and Theoretical Polymer Science 8, 229-246 (1998).

315 Sun, H. COMPASS: an ab initio force-field optimized for condensed-phase applications overview with details on alkane and benzene compounds. The Journal of Physical Chemistry B 102, 7338-7364 (1998).

316 Gaussian 16 Rev. C.01 (Wallingford, CT, 2016).

317 Nosé, S. A unified formulation of the constant temperature molecular dynamics methods. The Journal of chemical physics 81, 511-519 (1984).

318 Barton, A. F. Handbook of polymer-liquid interaction parameters and solubility parameters. (CRC press, 1990).

319 Schenderlein, S., Lück, M. \& Müller, B. Partial solubility parameters of poly (D, Llactide-co-glycolide). International journal of pharmaceutics 286, 19-26 (2004). 
320 Van Krevelen, D. W. \& Te Nijenhuis, K. Properties of polymers: their correlation with chemical structure; their numerical estimation and prediction from additive group contributions. (Elsevier, 2009).

321 Gupta, J., Nunes, C., Vyas, S. \& Jonnalagadda, S. Prediction of solubility parameters and miscibility of pharmaceutical compounds by molecular dynamics simulations. The Journal of Physical Chemistry B 115, 2014-2023 (2011).

322 Moghanloo, R. G., Davudov, D. \& Akita, E. in Formation Damage During Improved Oil Recovery (eds Bin Yuan \& David A. Wood) 243-273 (Gulf Professional Publishing, 2018).

323 Menard, J. P. Antibacterial treatment of bacterial vaginosis: current and emerging therapies. Int J Womens Health 3, 295-305, doi:10.2147/IJWH.S23814 (2011).

324 Castro, J. et al. Reciprocal interference between Lactobacillus spp. and Gardnerella vaginalis on initial adherence to epithelial cells. Int J Med Sci 10, 11931198, doi:10.7150/jms.6304 (2013).

325 Hawes, S. E. et al. Hydrogen peroxide-producing lactobacilli and acquisition of vaginal infections. J Infect Dis 174, 1058-1063 (1996).

326 Machado, D., Castro, J., Palmeira-de-Oliveira, A., Martinez-de-Oliveira, J. \& Cerca, N. Bacterial Vaginosis Biofilms: Challenges to Current Therapies and Emerging Solutions. Frontiers in microbiology 6, 1528, doi:10.3389/fmicb.2015.01528 (2015).

327 Vuotto, C., Longo, F. \& Donelli, G. Probiotics to counteract biofilm-associated infections: promising and conflicting data. Int J Oral Sci 6, 189-194, doi:10.1038/ijos.2014.52 (2014).

328 Homayouni, A. et al. Effects of probiotics on the recurrence of bacterial vaginosis: a review. Journal of lower genital tract disease 18, 79-86, doi:10.1097/LGT.0b013e31829156ec (2014).

329 Shalev, E., Battino, S., Weiner, E., Colodner, R. \& Keness, Y. Ingestion of yogurt containing Lactobacillus acidophilus compared with pasteurized yogurt as prophylaxis for recurrent candidal vaginitis and bacterial vaginosis. Archives of Family Medicine 5, 593 (1996).

330 Larsson, P.-G., Stray-Pedersen, B., Ryttig, K. R. \& Larsen, S. Human lactobacilli as supplementation of clindamycin to patients with bacterial vaginosis reduce the recurrence rate; a 6-month, double-blind, randomized, placebo-controlled study. $B M C$ women's health 8, 3 (2008).

331 Jang, S.-E. et al. Lactobacillus rhamnosus HN001 and Lactobacillus acidophilus La-14 attenuate Gardnerella vaginalis-infected bacterial vaginosis in mice. Nutrients 9, 531 (2017).

332 Paek, N.-S., Lee, Y. Y., Han, S. H., Kang, C.-H. \& So, J.-S. Characterization and inhibitory activity of Lactobacillus plantarum MG989 and Lactobacillus fermentum MG901 isolated from vaginal microbiota of Korean women against Gardnerella vaginalis and Candida albicans. KSBB Journal 31, 40-45 (2016).

333 Martinez, R. C. et al. Improved cure of bacterial vaginosis with single dose of tinidazole (2 g), Lactobacillus rhamnosus GR-1, and Lactobacillus reuteri RC-14: a randomized, double-blind, placebo-controlled trial. Canadian Journal of Microbiology 55, 133-138 (2009).

334 Ya, W., Reifer, C. \& Miller, L. E. Efficacy of vaginal probiotic capsules for recurrent bacterial vaginosis: a double-blind, randomized, placebo-controlled study. American journal of obstetrics and gynecology 203, 120. e121-120. e126 (2010).

335 MacPhee, R. A., Hummelen, R., Bisanz, J. E., Miller, W. L. \& Reid, G. Probiotic strategies for the treatment and prevention of bacterial vaginosis. Expert opinion on pharmacotherapy 11, 2985-2995 (2010). 
336 Anukam, K. C. et al. Clinical study comparing probiotic Lactobacillus GR-1 and $\mathrm{RC}-14$ with metronidazole vaginal gel to treat symptomatic bacterial vaginosis. Microbes and Infection 8, 2772-2776 (2006).

337 Mastromarino, P. et al. Effectiveness of Lactobacillus-containing vaginal tablets in the treatment of symptomatic bacterial vaginosis. Clinical microbiology and infection 15, 67-74 (2009).

338 Ehrström, S. et al. Lactic acid bacteria colonization and clinical outcome after probiotic supplementation in conventionally treated bacterial vaginosis and vulvovaginal candidiasis. Microbes and infection 12, 691-699 (2010).

339 Parent, D. et al. Therapy of bacterial vaginosis using exogenously-applied Lactobacilli acidophili and a low dose of estriol: a placebo-controlled multicentric clinical trial. Arzneimittel-Forschung 46, 68-73 (1996).

340 Gunawardana, M. et al. Sustained delivery of commensal bacteria from podintravaginal rings. Antimicrob Agents Ch 58, 2262-2267 (2014).

341 Tyo, K. M. et al. Relating advanced electrospun fiber architectures to the temporal release of active agents to meet the needs of next-generation intravaginal delivery applications. Pharmaceutics 11, 160 (2019).

342 Nagy, Z. K. et al. Nanofibrous solid dosage form of living bacteria prepared by electrospinning. (2014).

343 Zupančič, Š., Škrlec, K., Kocbek, P., Kristl, J. \& Berlec, A. Effects of Electrospinning on the Viability of Ten Species of Lactic Acid Bacteria in Poly (Ethylene Oxide) Nanofibers. Pharmaceutics 11, 483 (2019).

344 Salalha, W., Kuhn, J., Dror, Y. \& Zussman, E. Encapsulation of bacteria and viruses in electrospun nanofibres. Nanotechnology 17, 4675 (2006).

345 Liu, Y., Rafailovich, M. H., Malal, R., Cohn, D. \& Chidambaram, D. Engineering of bio-hybrid materials by electrospinning polymer-microbe fibers. Proceedings of the National Academy of Sciences 106, 14201-14206 (2009).

346 Chou, S.-F., Carson, D. \& Woodrow, K. A. Current strategies for sustaining drug release from electrospun nanofibers. Journal of Controlled Release 220, 584-591 (2015).

347 Amna, T., Hassan, M. S., Pandeya, D. R., Khil, M.-S. \& Hwang, I. Classy nonwovens based on animate L. gasseri-inanimate poly (vinyl alcohol): upstream application in food engineering. Applied microbiology and biotechnology 97, 45234531 (2013).

348 Tyo, K. M. et al. Multipurpose tenofovir disoproxil fumarate electrospun fibers for the prevention of HIV-1 and HSV-2 infections in vitro. International journal of pharmaceutics 531, 118-133 (2017).

349 Tyo, K. M. et al. Rapid-Release Griffithsin Fibers for the Dual Prevention of HSV2 and HIV-1 Infections. Antimicrob Agents Ch (2020).

350 Tyo, K. M. et al. pH-responsive delivery of Griffithsin from electrospun fibers. European Journal of Pharmaceutics and Biopharmaceutics 138, 64-74 (2019).

351 Mahmoud, M. Y., Sapare, S., Curry, K. C., Demuth, D. R. \& Steinbach-Rankins, J. M. Rapid Release Polymeric Fibers for Inhibition of Porphyromonas gingivalis Adherence to Streptococcus gordonii. Frontiers in Chemistry 7 (2019).

352 Emami, J. \& Rezazadeh, M. Rapid, sensitive, and validated HPLC method for analysis of metronidazole and tinidazole under identical chromatographic conditions with UV detection and liquid-liquid extraction: application in bioequivalence studies. Acta Chromatographica 25, 111-125 (2013).

353 Breshears, L. M., Edwards, V. L., Ravel, J. \& Peterson, M. L. Lactobacillus crispatus inhibits growth of Gardnerella vaginalis and Neisseria gonorrhoeae on a porcine vaginal mucosa model. BMC microbiology 15, 276 (2015). 
354 Santos, C. M. et al. Anti-inflammatory effect of two Lactobacillus strains during infection with Gardnerella vaginalis and Candida albicans in a HeLa cell culture model. Microbiology 164, 349-358 (2018).

355 Yari, M., Fooladi, J. \& Motlagh, M. A. K. Microencapsulation and Fermentation of Lactobacillus acidophilus LA-5 and Bifidobacterium BB-12. Applied Food Biotechnology 2, 27-32 (2015).

356 Atassi, F., Brassart, D., Grob, P., Graf, F. \& Servin, A. L. Lactobacillus strains isolated from the vaginal microbiota of healthy women inhibit Prevotella bivia and Gardnerella vaginalis in coculture and cell culture. FEMS Immunology \& Medical Microbiology 48, 424-432 (2006).

357 Strus, M., Malinowska, M. \& Heczko, P. B. In vitro antagonistic effect of Lactobacillus on organisms associated with bacterial vaginosis. The Journal of reproductive medicine 47, 41-46 (2002).

358 Atassi, F., Ahn, P. V., Diane, L. \& Moal, L.-L. Diverse expression of antimicrobial activities against bacterial vaginosis and urinary tract infection pathogens by cervicovaginal microbiota strains of Lactobacillus gasseri and Lactobacillus crispatus. Frontiers in microbiology 10, 2900 (2019).

359 Chetwin, E. et al. Antimicrobial and inflammatory properties of South African clinical Lactobacillus isolates and vaginal probiotics. Scientific reports 9, 1917 (2019).

360 Gahlot, N. \& Maheshwari, R. K. Formulation and development of vaginal films of poorly water soluble drug, metronidazole, using mixed solvency concept and their evaluations. Journal of Drug Delivery and Therapeutics 8, 41-48 (2018).

361 Blakney, A. K., Ball, C., Krogstad, E. A. \& Woodrow, K. A. Electrospun fibers for vaginal anti-HIV drug delivery. Antiviral research 100, S9-S16 (2013).

362 Blakney, A. K., Jiang, Y. \& Woodrow, K. A. Application of electrospun fibers for female reproductive health. Drug delivery and translational research 7, 796-804 (2017).

363 Fung, W.-Y., Yuen, K.-H. \& Liong, M.-T. Agrowaste-based nanofibers as a probiotic encapsulant: fabrication and characterization. Journal of agricultural and food chemistry 59, 8140-8147 (2011).

364 Ghorani, B. \& Tucker, N. Fundamentals of electrospinning as a novel delivery vehicle for bioactive compounds in food nanotechnology. Food Hydrocolloids 51, 227-240 (2015).

365 Soares, J., da Costa, M. M. \& de Oliveira, H. P. Encapsulation and Bioavailability of Lactobacillus spp. in Electrospun Fibers. Current Biotechnology 9, 15-22 (2020).

366 Strasser, S., Neureiter, M., Geppl, M., Braun, R. \& Danner, H. Influence of lyophilization, fluidized bed drying, addition of protectants, and storage on the viability of lactic acid bacteria. Journal of Applied Microbiology 107, 167-177 (2009).

367 Jalali, M. et al. Stability evaluation of freeze-dried Lactobacillus paracasei subsp. tolerance and Lactobacillus delbrueckii subsp. bulgaricus in oral capsules. Research in pharmaceutical sciences 7, 31 (2012).

368 Poirier, I., Maréchal, P. A., Richard, S. \& Gervais, P. Saccharomyces cerevisiae viability is strongly dependant on rehydration kinetics and the temperature of dried cells. Journal of Applied Microbiology 86, 87-92 (1999).

369 Heunis, T., Botes, M. \& Dicks, L. Encapsulation of Lactobacillus plantarum 423 and its bacteriocin in nanofibers. Probiotics and antimicrobial proteins 2, 46-51 (2010).

370 Wongsasulak, S., Tongsin, P., Intasanta, N. \& Yoovidhya, T. Effect of glycerol on solution properties governing morphology, glass transition temperature, and 
tensile properties of electrospun zein film. Journal of applied polymer science 118, 910-919 (2010).

371 Avci, H., Monticello, R. \& Kotek, R. Preparation of antibacterial PVA and PEO nanofibers containing Lawsonia Inermis (henna) leaf extracts. Journal of Biomaterials Science, Polymer Edition 24, 1815-1830 (2013).

372 Tsai, C.-C., Lai, T.-M. \& Hsieh, Y.-M. Evaluation of Lactobacilli for Antagonistic Activity Against the Growth, Adhesion and Invasion of Klebsiella pneumoniae and Gardnerella vaginalis. Indian journal of microbiology 59, 81-89 (2019).

373 Coudeyras, S., Jugie, G., Vermerie, M. \& Forestier, C. Adhesion of human probiotic Lactobacillus rhamnosus to cervical and vaginal cells and interaction with vaginosis-associated pathogens. Infectious diseases in obstetrics and gynecology 2008 (2008).

374 Falagas, M., Betsi, G. \& Athanasiou, S. Probiotics for the treatment of women with bacterial vaginosis. Clinical microbiology and infection 13, 657-664 (2007).

375 Allsworth, J. E. \& Peipert, J. F. Prevalence of bacterial vaginosis: 2001-2004 National Health and Nutrition Examination Survey data. Obstetrics and gynecology 109, 114-120, doi:10.1097/01.AOG.0000247627.84791.91 (2007).

376 Kenyon, C., Colebunders, R. \& Crucitti, T. The global epidemiology of bacterial vaginosis: a systematic review. American journal of obstetrics and gynecology 209, 505-523, doi:10.1016/j.ajog.2013.05.006 (2013).

377 Mastromarino, P., Vitali, B. \& Mosca, L. Bacterial vaginosis: a review on clinical trials with probiotics. The new microbiologica 36, 229-238 (2013).

378 Verstraelen, H., Vervaet, C. \& Remon, J. P. Rationale and Safety Assessment of a Novel Intravaginal Drug-Delivery System with Sustained DL-Lactic Acid Release, Intended for Long-Term Protection of the Vaginal Microbiome. PloS one 11, e0153441, doi:10.1371/journal.pone.0153441 (2016).

379 Ejike, C., Agbakoba, N., Ezeanya, C. \& Anukam, K. Health, Social and Economic burden of Bacterial vaginosis (BV) among Nigerian women of child bearing age: Can Probiotics restore the vaginal dysbiosis. J Med Lab Sci 29, 37-48 (2019).

380 Martin, D. H. \& Marrazzo, J. M. The Vaginal Microbiome: Current Understanding and Future Directions. J Infect Dis 214 Suppl 1, S36-41, doi:10.1093/infdis/jiw184 (2016).

381 Nasioudis, D., Linhares, I. M., Ledger, W. J. \& Witkin, S. S. Bacterial vaginosis: a critical analysis of current knowledge. BJOG : an international journal of obstetrics and gynaecology, doi:10.1111/1471-0528.14209 (2016).

382 Martin, H. L. et al. Vaginal lactobacilli, microbial flora, and risk of human immunodeficiency virus type 1 and sexually transmitted disease acquisition. $J$ Infect Dis 180, 1863-1868, doi:10.1086/315127 (1999).

383 Taha, T. E. et al. Bacterial vaginosis and disturbances of vaginal flora: association with increased acquisition of HIV. Aids 12, 1699-1706 (1998).

384 Ngugi, B. M. et al. Effects of BV-associated bacteria and sexual intercourse on vaginal colonization with the probiotic Lactobacillus crispatus CTV-05. Sexually transmitted diseases 38, 1020 (2011).

385 Basavaprabhu, H., Sonu, K. \& Prabha, R. Mechanistic insights into the action of probiotics against bacterial vaginosis and its mediated preterm birth: An overview. Microbial Pathogenesis, 104029 (2020).

386 Menard, J.-P. Antibacterial treatment of bacterial vaginosis: current and emerging therapies. International journal of women's health 3, 295 (2011).

387 Fichorova, R., Yamamoto, H., Delaney, M., Onderdonk, A. \& Doncel, G. Novel vaginal microflora colonization model providing new insight into microbicide mechanism of action. mBio 2, e00168-00111 (2011). 
388 Aroutcheva, A. et al. Defense factors of vaginal lactobacilli. American journal of obstetrics and gynecology 185, 375-379, doi:10.1067/mob.2001.115867 (2001).

389 Witkin, S. S. The vaginal microbiome, vaginal anti-microbial defence mechanisms and the clinical challenge of reducing infection-related preterm birth. BJOG : an international journal of obstetrics and gynaecology 122, 213-218, doi:10.1111/1471-0528.13115 (2015).

390 Bohbot, J. et al. Efficacy and safety of vaginally administered lyophilized Lactobacillus crispatus IP 174178 in the prevention of bacterial vaginosis recurrence. Journal of gynecology obstetrics and human reproduction 47, 81-86 (2018).

391 Hemmerling, A. et al. Phase 1 dose-ranging safety trial of Lactobacillus crispatus CTV-05 (LACTIN-V) for the prevention of bacterial vaginosis. Sexually transmitted diseases 36, 564 (2009).

392 Hemmerling, A. et al. Phase 2a study assessing colonization efficiency, safety, and acceptability of Lactobacillus crispatus CTV-05 in women with bacterial vaginosis. Sexually transmitted diseases 37, 745-750 (2010).

393 McLEAN, N. W. \& ROSENSTEIN, I. J. Characterisation and selection of a Lactobacillus species to re-colonise the vagina of women with recurrent bacterial vaginosis. Journal of medical microbiology 49, 543-552 (2000).

394 Fredricsson, B., Englund, K., Weintraub, L., Ölund, A. \& Nord, C.-E. Bacterial vaginosis is not a simple ecological disorder. Gynecologic and obstetric investigation 28, 156-160 (1989).

395 Ozkinay, E. et al. The effectiveness of live lactobacilli in combination with low dose oestriol (Gynoflor) to restore the vaginal flora after treatment of vaginal infections. BJOG: An International Journal of Obstetrics \& Gynaecology 112, 234-240 (2005).

396 Hallén, A., Jarstrand, C. \& Påhlson, C. Treatment of bacterial vaginosis with lactobacilli. Sexually transmitted diseases 19, 146-148 (1992).

397 Lev-Sagie, A. et al. Vaginal microbiome transplantation in women with intractable bacterial vaginosis. Nature medicine 25, 1500-1504 (2019).

398 Ma, D., Chen, Y. \& Chen, T. Vaginal microbiota transplantation for the treatment of bacterial vaginosis: a conceptual analysis. FEMS microbiology letters 366, fnz025 (2019).

399 Lev-Sagie, A. et al. Vaginal microbiome transplantation in women with intractable bacterial vaginosis. Nat Med 25, 1500-1504, doi:10.1038/s41591-019-0600-6 (2019).

400 DeLong, K. et al. Conceptual design of a universal donor screening approach for vaginal microbiota transplant. Frontiers in cellular and infection microbiology 9, 306 (2019).

401 Patel, D. B. \& Patel, J. K. Liposomal drug delivery of metronidazole for the local treatment of vaginitis. Int. J. Pharm. Sci. Nanotechnol 2, 248-257 (2009).

402 Handalishy, I. I., Behery, M. A., Elkhouly, M., Farag, E. A. \& Elsheikh, W. A. Comparative study between probiotic vaginal tampons and oral metronidazole in treatment of bacterial vaginosis. AAMJ 12, 185-203 (2014).

403 Decena, D. C. et al. Metronidazole with Lactacyd vaginal gel in bacterial vaginosis. J Obstet Gynaecol Res 32, 243-251, doi:10.1111/j.1447-0756.2006.00383.x (2006).

404 Borges, S., Silva, J. \& Teixeira, P. The role of lactobacilli and probiotics in maintaining vaginal health. Archives of gynecology and obstetrics 289, 479-489 (2014).

405 Lai, S. K., Wang, Y. Y., Hida, K., Cone, R. \& Hanes, J. Nanoparticles reveal that human cervicovaginal mucus is riddled with pores larger than viruses. Proceedings 
of the National Academy of Sciences of the United States of America 107, 598603, doi:10.1073/pnas.0911748107 (2010).

406 Baelo, A. et al. Disassembling bacterial extracellular matrix with DNase-coated nanoparticles to enhance antibiotic delivery in biofilm infections. Journal of controlled release : official journal of the Controlled Release Society 209, 150-158, doi:10.1016/j.jconrel.2015.04.028 (2015).

407 Camacho, D. P. et al. Vaginal yeast adherence to the combined contraceptive vaginal ring (CCVR). Contraception 76, 439-443 (2007).

408 Gunawardana, M. et al. Microbial biofilms on the surface of intravaginal rings worn in non-human primates. Journal of medical microbiology 60, 828 (2011).

409 Grooms, T. N. et al. Griffithsin-Modified Electrospun Fibers as a Delivery Scaffold to Prevent HIV Infection. Antimicrobial agents and chemotherapy, doi:10.1128/AAC.00956-16 (2016).

410 Huang, C. et al. Electrospun polystyrene fibers for HIV entrapment. Polymers Advanced Technologies 25, 827-834 (2014).

411 Vasita, R. \& Katti, D. S. Nanofibers and their applications in tissue engineering. Int $J$ Nanomedicine 1, 15-30 (2006).

412 Vuong, H. R., Tyo, K. M. \& Steinbach-Rankins, J. M. Fabrication and Characterization of Griffithsin-Modified Fiber Scaffolds for STI Prevention. Journal of Visual Experiments accepted, August 2017. (2017).

413 Kai, D., Liow, S. S. \& Loh, X. J. Biodegradable polymers for electrospinning: towards biomedical applications. Mater Sci Eng C Mater Biol Appl 45, 659-670, doi:10.1016/j.msec.2014.04.051 (2014).

414 Samprasit, W. et al. Fabrication and In Vitro/In Vivo Performance of Mucoadhesive Electrospun Nanofiber Mats Containing alpha-Mangostin. AAPS PharmSciTech 16, 1140-1152, doi:10.1208/s12249-015-0300-6 (2015).

415 Zussman, E. Encapsulation of cells within electrospun fibers. Polymers for Advanced Technologies 22, 366-371 (2011).

416 Farnaz Minooei, L. Z., Mary Sarah NeCamp, Ruta Patwardhan, Kevin M. Tyo, Walter H. Watson, Nicole M. Gilbert, Amanda L. Lewis, Jill M. Steinbach-Rankins. Probiotic fibers as an alternative dosage form for bacterial vaginosis treatment. Submitted in Science Advances (2020).

417 Garcia, E. M., Kraskauskiene, V., Koblinski, J. E. \& Jefferson, K. K. Interaction of Gardnerella vaginalis and vaginolysin with the apical versus basolateral face of a three-dimensional model of vaginal epithelium. Infection and immunity 87, e0064600618 (2019).

418 Sieber, R. \& Dietz, U.-T. Lactobacillus acidophilus and yogurt in the prevention and therapy of bacterial vaginosis. International Dairy Journal 8, 599-607 (1998).

419 Reid, G. et al. Oral use of Lactobacillus rhamnosus GR-1 and L. fermentum RC14 significantly alters vaginal flora: randomized, placebo-controlled trial in 64 healthy women. FEMS Immunology \& Medical Microbiology 35, 131-134 (2003).

420 Eriksson, K., Carlsson, B., Forsum, U. \& Larsson, P. A double-blind treatment study of bacterial vaginosis with normal vaginal lactobacilli after an open treatment with vaginal clindamycin ovules. Acta dermato-venereologica 85, 42-46 (2005).

421 Carson, D., Jiang, Y. \& Woodrow, K. A. Tunable release of multiclass anti-HIV drugs that are water-soluble and loaded at high drug content in polyester blended electrospun fibers. Pharmaceutical research 33, 125-136 (2016).

422 Ball, C., Chou, S.-F., Jiang, Y. \& Woodrow, K. A. Coaxially electrospun fiber-based microbicides facilitate broadly tunable release of maraviroc. Materials Science and Engineering: C 63, 117-124 (2016). 
423 Zolnik, B. S. \& Burgess, D. J. Effect of acidic pH on PLGA microsphere degradation and release. Journal of Controlled Release 122, 338-344 (2007).

424 Evrova, O. et al. Hybrid randomly electrospun poly (lactic-co-glycolic acid): poly (ethylene oxide)(PLGA: PEO) fibrous scaffolds enhancing myoblast differentiation and alignment. ACS applied materials \& interfaces 8, 31574-31586 (2016).

425 Reid, G., Beuerman, D., Heinemann, C. \& Bruce, A. W. Probiotic Lactobacillus dose required to restore and maintain a normal vaginal flora. FEMS Immunology \& Medical Microbiology 32, 37-41 (2001).

426 Pandey, V. K. et al. Differential susceptibility of catheter biomaterials to biofilmassociated infections and their remedy by drug-encapsulated Eudragit RL100 nanoparticles. International journal of molecular sciences 20, 5110 (2019).

427 Amabebe, E. \& Anumba, D. O. The vaginal microenvironment: the physiologic role of lactobacilli. Frontiers in medicine 5, 181 (2018).

428 Tachedjian, G., Aldunate, M., Bradshaw, C. S. \& Cone, R. A. The role of lactic acid production by probiotic Lactobacillus species in vaginal health. Research in microbiology 168, 782-792 (2017).

429 Witkin, S. S. et al. Influence of vaginal bacteria and D-and L-lactic acid isomers on vaginal extracellular matrix metalloproteinase inducer: implications for protection against upper genital tract infections. MBio 4, e00460-00413 (2013).

430 O'Hanlon, D. E., Moench, T. R. \& Cone, R. A. Vaginal pH and microbicidal lactic acid when lactobacilli dominate the microbiota. PloS one 8, e80074 (2013).

431 Osset, J., Bartolomé, R. M., García, E. \& Andreu, A. Assessment of the capacity of Lactobacillus to inhibit the growth of uropathogens and block their adhesion to vaginal epithelial cells. The Journal of infectious diseases 183, 485-491 (2001).

432 Atassi, F., Brassart, D., Grob, P., Graf, F. \& Servin, A. In vitro antibacterial activity of Lactobacillus helveticus strain KS300 against diarrhoeagenic, uropathogenic and vaginosis-associated bacteria. Journal of applied microbiology 101, 647-654 (2006).

433 Tyo, K. M. et al. Rapid-Release Griffithsin Fibers for Dual Prevention of HSV-2 and HIV-1 Infections. Antimicrobial Agents and Chemotherapy 64 (2020).

434 Meng, J. et al. Tenofovir containing thiolated chitosan core/shell nanofibers: in vitro and in vivo evaluations. Molecular Pharmaceutics 13, 4129-4140 (2016).

435 Fernández-Romero, J. A. et al. Multipurpose prevention technologies: the future of HIV and STI protection. Trends in microbiology 23, 429-436 (2015).

436 Ayub, M. \& Bayley, H. Engineered transmembrane pores. Current opinion in chemical biology 34, 117-126 (2016).

437 Howorka, S. Building membrane nanopores. Nature Nanotechnology 12, 619-630 (2017).

438 Schlesinger, P. H. et al. Anchor chain length alters the apparent mechanism of chloride channel function in SCMTR derivatives. Chemical Communications, 308309, doi:10.1039/b211629b (2003).

439 Schlesinger, P. H. et al. SCMTR: A Chloride-Selective, Membrane-Anchored Peptide Channel that Exhibits Voltage Gating. Journal of the American Chemical Society 124, 1848-1849, doi:10.1021/ja016784d (2002).

440 Gokel, G. W. \& Negin, S. Synthetic membrane active amphiphiles. Advanced drug delivery reviews 64, 784-796 (2012).

441 Pajewski, R. et al. The effect of midpolar regime mimics on anion transport mediated by amphiphilic heptapeptides. New Journal of Chemistry 31, 1960-1972 (2007).

442 Fahlke, C., Rhodes, T. H., Desai, R. R. \& George Jr, A. L. Pore stoichiometry of a voltage-gated chloride channel. Nature 394, 687 (1998). 
443 Gokel, G. W. \& Daschbach, M. M. in Anion Coordination Chemistry

(Wiley-VCH Verlag GmbH \& Co. KGaA, 2011).

444 Ferdani, R. \& Gokel, G. W. Planar bilayer studies reveal multiple conductance states for synthetic anion transporters. Organic \& Biomolecular Chemistry 4, 37463750, doi:10.1039/B609345K (2006).

445 Burkhardt, J. B. Computational Modeling of SCMTR: A Synthetic Anion Channel, University of Cincinnati, (2013).

446 Djedovic, N. et al. The C-terminal ester of membrane anchored peptide ion channels affects anion transport. Chemical Communications, 2862-2863, doi:10.1039/B312209N (2003).

447 Schlesinger, P. H., Ferdani, R., Pajewska, J., Pajewski, R. \& Gokel, G. W. Replacing proline at the apex of heptapeptide-based chloride ion transporters alters their properties and their ionophoretic efficacy. New Journal of Chemistry 27, 60-67 (2003).

448 Djedovic, N. et al. The C-and N-terminal residues of synthetic heptapeptide ion channels influence transport efficacy through phospholipid bilayers. New Journal of Chemistry 29, 291-305, doi:10.1039/B417091C (2005).

449 Ferdani, R. et al. Transport of chloride and carboxyfluorescein through phospholipid vesicle membranes by heptapeptide amphiphiles. Organic \& biomolecular chemistry 5, 2423-2432 (2007).

450 Pajewski, R. et al. Evidence for dimer formation by an amphiphilic heptapeptide that mediates chloride and carboxyfluorescein release from liposomes. Organic \& Biomolecular Chemistry 3, 619-625, doi:10.1039/B417009A (2005).

451 Daschbach, M. M., Negin, S., You, L., Walsh, M. \& Gokel, G. W. Aggregation and Supramolecular Membrane Interactions that Influence Anion Transport in Tryptophan-Containing Synthetic Peptides. Chemistry-A European Journal 18, 7608-7623 (2012).

452 Martin, M., Dubbs, T. \& Fried, J. R. Planar Bilayer Measurements of Alamethicon and Gramicidin Reconstituted in Biomimetic Block Copolymers. Langmuir 33, 1171-1179 (2017).

453 Wong, D., Jeon, T.-J. \& Schmidt, J. Single molecule measurements of channel proteins incorporated into biomimetic polymer membranes. Nanotechnology 17, 3710-3717, doi:10.1088/0957-4484/17/15/016 (2006).

454 Burkhardt, J. B., Skelton, A. A. \& Fried, J. R. The water-channel forming ability of heptapeptide-based anion channels: insights from molecular dynamics simulations. Soft Matter 9, 4444-4454, doi:10.1039/C3SM00061C (2013).

455 Temmerman, M., Khosla, R., Laski, L., Mathews, Z. \& Say, L. Women's health priorities and interventions. BMJ : British Medical Journal 351, doi:10.1136/bmj.h4147 (2015).

456 Decker, J. S., Menacho-Melgar, R. \& Lynch, M. D. Low-Cost, Large-Scale Production of the Anti-viral Lectin Griffithsin. Frontiers in bioengineering and biotechnology 8, 1020 (2020).

457 Moulaei, T. et al. Monomerization of viral entry inhibitor griffithsin elucidates the relationship between multivalent binding to carbohydrates and anti-HIV activity. Structure 18, 1104-1115, doi:10.1016/j.str.2010.05.016 (2010).

458 Vujic, G., Knez, A. J., Stefanovic, V. D. \& Vrbanovic, V. K. Efficacy of orally applied probiotic capsules for bacterial vaginosis and other vaginal infections: a doubleblind, randomized, placebo-controlled study. European Journal of Obstetrics \& Gynecology and Reproductive Biology 168, $75-79$ (2013). 


\title{
CURRICULUM VITAE
}

\author{
Farnaz Minooei \\ Farnaz.minooei@louisville.edu
}

502-975-0445

\section{Education:}

- Ph.D., Chemical Engineering

August 2016-December 2020

University of Louisville Louisville, KY

- Dissertation: "The Use of Nanoparticles and Electrospun Fibers for Intravaginal Delivery to Treat Viral and Bacterial Infections and Electrophysiological Measurements of Synthetic Chloride Channels" Dr. Joel Fried Dr. Steinbach-Rankins

- B.S., Chemical Engineering Sharif University of Technology

- Dissertation: "Applied Approaches to Enhancing Mechanical Properties of Hydrogels in Tissue Engineering"

Dr. Shohre Mashayekhan

\section{Research Experience:}

- Graduate Student Aug 2016-current Chemical and Bioengineering, University of Louisville Louisville, KY

- Successfully implemented computational modeling to verify experimental results of encapsulation efficacies of polymeric nanoparticles encapsulating antiretrovirals.

- Designed polymeric nanoparticle formulations encapsulating combinations of biologic-antiretrovirals that demonstrated an 8-fold increase in antiviral efficacy against HIV-1.

- Developed novel dual-spun and multilayered polymeric fiber delivery platforms that provided sustained-release of probiotics up to 14 days for use against bacterial vaginosis. 
- Successfully troubleshooted delivery platform manufacturing operations by implementing fabrication procedures that ensured sterility, maintained purity of incorporated probiotics, and prevented contamination.

- Managed lab operations including ordering and maintaining supplies, as well as maintaining and troubleshooting laboratory equipment.

- Guest Researcher

September 2014- May 2016

Chemical Engineering, Tarbiat Modares University

Tehran, Iran

- Conducted research on techniques for improving the performance of polymeric membranes for protein separation and purification; resulted in a first-author publication.

- Collaborated with other groups in different universities to accomplish project goals.

Work Experience:

- Teaching Assistant

August 2018-August 2019 Chemical Engineering, University of Louisville Louisville, KY

- Selected as a teaching assistant for 4 core chemical engineering courses.

- Supervised and trained students in lab sessions and graded lab reports.

- Assisted with the design of course material, grading exams and homework.

- Teaching Assistant

August 2015-December 2015

Chemical Engineering, Sharif University of Technology Tehran, Iran

- Assisted with curriculum development and grading exams and presentations.

\section{Honors and Awards:}

Dissertation Completion Award from

July 2020-Dec 2020

Graduate School at University of Louisville

Student Board Representative at CRS

Jan 2019-Current

$1^{\text {st }}$ place Research Louisville poster award in Engineering

September 2019

Travel awards from the Graduate Student Council (GSC)

2018 and 2019

Ph.D. Fellowship recipient from

August 2016-August 2018 
Chemical Engineering department at University of Louisville

\section{Publications:}

- Minooei F; Kanukunta A.R; Lewis A.L; Steinbach-Rankins, J.M. Mesh and Layered Fiber Architectures as Novel Platforms to Sustain Probiotic Release against Bacterial Vaginosis Infection, (manuscript in preparation).

- Minooei F; Fried J.R.; Fuqua J.L.; Palmer K.E.; Steinbach-Rankins, J.M. In Vitro Study on Synergistic Interactions between Different Antiretrovirals and QGriffithsin against HIV-1, (manuscript in preparation).

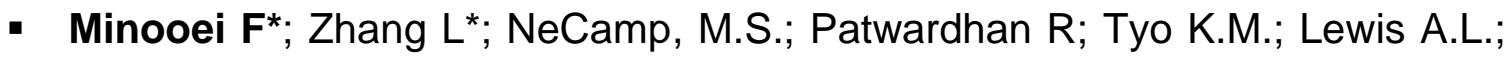
Steinbach-Rankins J.M. Probiotic Electrospun Fibers for Bacterial Vaginosis Treatment, (manuscript in preparation).

- Minooei F; Steinbach-Rankins J.M; Fried J.R. A Computational Study of Interactions between Antiretrovirals and PLGA Nanoparticles, (manuscript in preparation).

- Chandrashekhar P*; Arreguin W.*; Minooei F.; Masiogol M.; SteinbachRankins J. M. New Delivery Vehicles for Intravaginal Probiotic Delivery, (manuscript in preparation).

- Desai H; Mahmoud M. Y.; Tan J; Minooei F; Demuth D. R.; Steinbach-Rankins J. M. Assessment of CafA Targeted BAR-Encapsulated Nanoparticles against Oral Biofilms, Pharmaceutics 2020 (Accepted).

- Tyo, K.M.*; Minooei F.*; Curry, K.C.; NeCamp, S.M.; Graves, D.L.; Fried, J.R.; Steinbach-Rankins, J.M. Relating Advanced Electrospun Fiber Architectures to the Temporal Release of Active Agents to Meet the Needs of Next-Generation Intravaginal Delivery Applications. Pharmaceutics 2019, 11, 160.

- Minooei $\mathrm{F}^{*}$, Martin $\mathrm{MD}^{*}$, Fried JR, Brian JP. Electrophysiological measurements reveal that a succinyl linker enhances the performance of the synthetic chloride channel SCMTR. Chemical Communications. 2018;54(37):4689-91.

- Minooei $\mathbf{F}^{\star}$, Vedadghavami $A^{*}$, Hosseini SS. Practical techniques for improving the performance of polymeric membranes and processes for protein separation and purification. Iranian Journal of Chemistry and Chemical Engineering (IJCCE). 2018 Apr 1;37(2):1-23.

- Minooei $\mathbf{F}^{*}$, Vedadghavami $A^{*}$, Mohammadi MH, Khetani S, Kolahchi AR, Mashayekhan S, Sanati-Nezhad A. Manufacturing of hydrogel biomaterials with controlled mechanical properties for tissue engineering applications. Acta Biomaterialia. 2017 Oct 15;62:42-63.

- *Shared first authorship

Poster Presentations and Invited Lectures: 


\section{- Oral Presentations}

- "Dual-spun Mesh Fibers as a Novel Platform to Sustain Vaginal Probiotic Release against Bacterial Vaginosis Infection" CRS virtual annual conference, June 2020.

- "Synergy of Antiretrovirals and Biologic Nanoparticles against HIV-1 Infection" GSC regional conference, Louisville, KY, February 2020.

- "Synergy of Antiretrovirals and Biologic Nanoparticles against HIV-1 Infection" KY Nano/AM symposium Louisville, Kentucky, 1 August 2019.

- "Planar Bilayer Experiments with Synthetic Anion Transporters in Model Membrane Systems" IBE conference, Norfolk, Virginia, 5-7 April 2018

\section{- Posters}

- "Synergistic Activity of Antiretroviral and Biologic Nanoparticles against HIV-1 Infection" BMES annual meeting, Philadelphia, Pennsylvania, 16-19 Oct 2019.

- "Synergistic Activity of Antiretroviral and Biologic Nanoparticles against HIV-1 Infection" Research Louisville, Louisville, Kentucky, 10 Sep 2019. 